\title{
Review of the General- Purpose Heat Source Module Reduction and Monitoring Processing at INL
}

\section{August 2020}

Joe Giglio

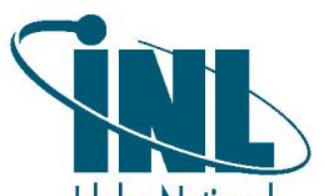

Idaho National

Laboratory 


\section{DISCLAIMER}

This information was prepared as an account of work sponsored by an agency of the U.S. Government. Neither the U.S. Government nor any agency thereof, nor any of their employees, makes any warranty, expressed or implied, or assumes any legal liability or responsibility for the accuracy, completeness, or usefulness, of any information, apparatus, product, or process disclosed, or represents that its use would not infringe privately owned rights. References herein to any specific commercial product, process, or service by trade name, trade mark, manufacturer, or otherwise, does not necessarily constitute or imply its endorsement, recommendation, or favoring by the U.S. Government or any agency thereof. The views and opinions of authors expressed herein do not necessarily state or reflect those of the U.S. Government or any agency thereof. 


\title{
Review of the General-Purpose Heat Source Module Reduction and Monitoring Processing at INL
}

\author{
Joseph Giglio
}

July 2020

\begin{abstract}
Idaho National Laboratory
Radioisotope Power Systems

Idaho Falls, Idaho 83415
\end{abstract}

http://www.inl.gov

\author{
Prepared for the \\ U.S. Department of Energy \\ Office of Nuclear Energy \\ Under DOE Idaho Operations Office \\ Contract DE-AC07-05ID14517
}


Page intentionally left blank 


\section{ABSTRACT}

A thorough review of the Module Reduction and Monitoring (MRM) process was conducted to evaluate the efficacy of processing. The degree of reduction was calculated using the mass balance of oxygen contained in carbon monoxide $(\mathrm{CO})$ and carbon dioxide $\left(\mathrm{CO}_{2}\right)$ removed from all fuel processed between January 2014 through August 2019. The plutonia was slightly reduced from O/Pu ratio of 2.0 to a range of 1.991 to 1.999. The review evaluated publications generated when MRM processing was developed and performed at the Mound Laboratory. A similar degree of reduction was measured at Mound in the 1982 paper authored by Ernie Johnson as well as MRM processing of IHS-60 and some Cassini-era fuel. The original 1982 paper documenting the reduction process reported a mass balance reduction to 1.9931 yet reported a total reduction to $\sim 1.98$ (Johnson 1990). The IHS-60 and Cassini reductions were calculated at 1.995 and 1.997 using the number of moles of $\mathrm{CO}$ and $\mathrm{CO}_{2}$ removed as published in a clad pressurization investigation report (Merten, et all 1995). MRM process changes are recommended to improve the accuracy of the $\mathrm{CO}$ partial pressure calcualtion. 
Page intentionally left blank 


\section{CONTENTS}

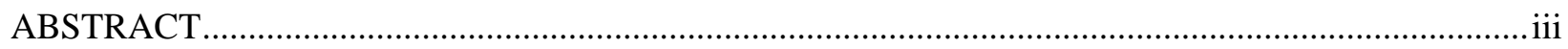

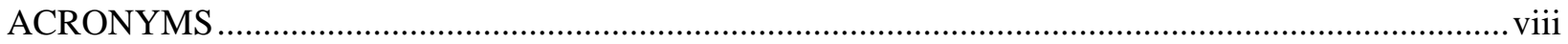

Review of the General-Purpose Heat Source Module Reduction and Monitoring Processing at INL ......... 1

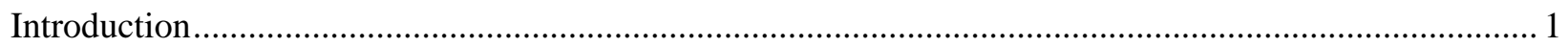

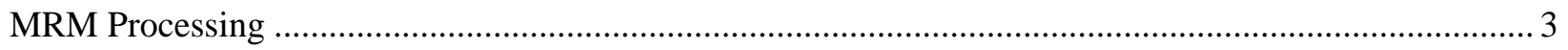

Estimating Can Pressures when Closed to the Manifold ................................................................. 7

Addition Comments to the Published Literature...................................................................... 8

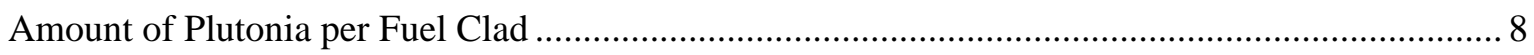

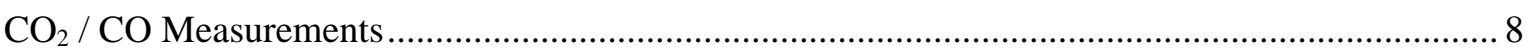

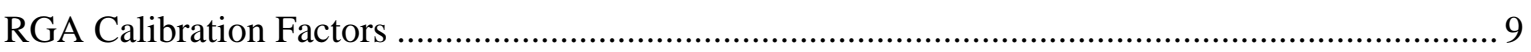

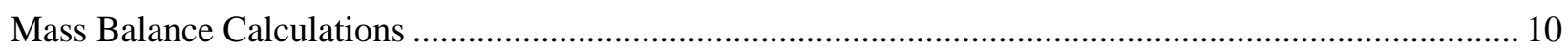

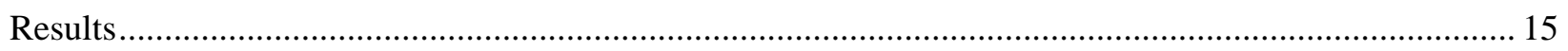

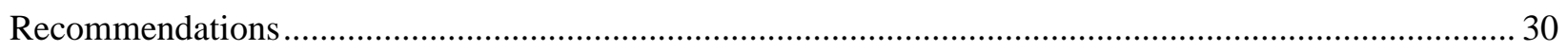

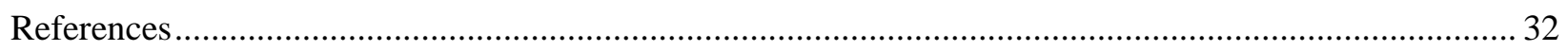

Appendix A Double release method of determining the initial gas pressure in an unknown volume at

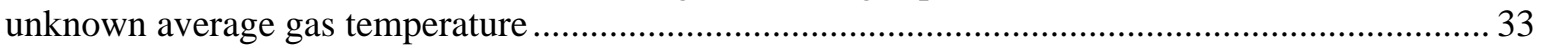

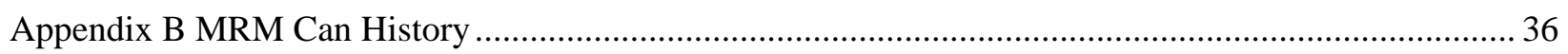

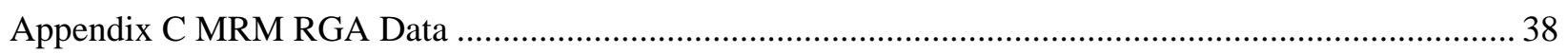

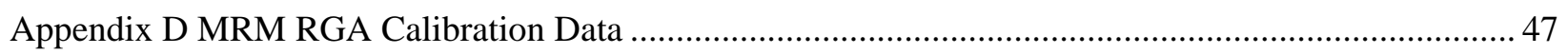

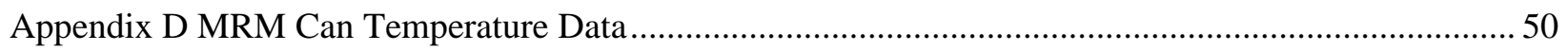

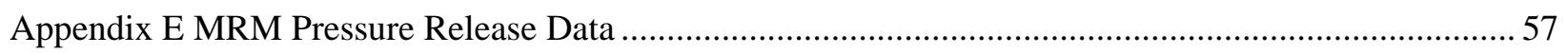

Appendix F MRM Can L1303 Reduction Calculation using the RGA Vacuum Pressure ........................ 63

Appendix G MRM Can L1303 Reduction Calculation using the Calibration Gas Sum of the RGA Partial

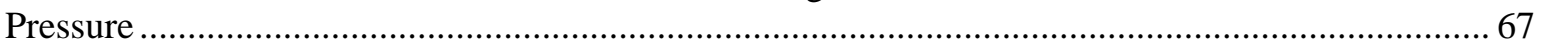

Appendix H MRM Can L1303 Reduction Calculation using the Fill Pressure and RGA Partial Pressure of

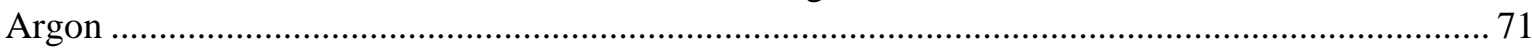

\section{FIGURES}

Figure 1. Investigation of Apparent Fuel Clad Pressurization IHS-60 MRM data

Figure 2. Investigation of Apparent Fuel Clad Pressurization Cassini MRM data 
Error! Reference source not found.

Error! Reference source not found.

Figure 3. MRMF with multiple cans processing

Figure 4. Packaging drawing of MRM can L134

Figure 5. Updated GIS packaging with 2 GIS post can cutting

Figure 6 - Calibration factors and ratio of $\mathrm{CO} / \mathrm{Ar}$ vs time

Figure 7 - April 27, 2107 RGA scan of can L1308

Figure 8 - MRM Can L126 CO Pressure and Stoichiometry vs Gas Tap

Figure 9 - MRM Can L134 CO Pressure and Stoichiometry vs Gas Tap

Figure 10 - MRM Can L1307 CO Pressure and Stoichiometry vs Gas Tap

Figure 11 - MRM Can L1308 CO Pressure and Stoichiometry vs Gas Tap

Figure 12 - MRM Can L1303 CO Pressure and Stoichiometry vs Gas Tap

Figure 13 - MRM Can L1701 CO Pressure and Stoichiometry vs Gas Tap

Figure 14 - MRM Can L1301 CO Pressure and Stoichiometry vs Gas Tap

Figure 15 - MRM Can L1306 CO Pressure and Stoichiometry vs Gas Tap

Figure 16 - MRM Can L1311 CO Pressure and Stoichiometry vs Gas Tap

Figure 17 - MRM Can L1702 CO Pressure and Stoichiometry vs Gas Tap

Figure 18 - MRM Can L1704 CO Pressure and Stoichiometry vs Gas Tap

Figure 19 - MRM Can L1707 CO Pressure and Stoichiometry vs Gas Tap

Figure 20 - MRM Can L1708 CO Pressure and Stoichiometry vs Gas Tap

Figure 21 - Calculated $\mathrm{O}_{2}$ partial pressure versus O/Pu ratio (Fig 11 Guéneau 2008)

\section{TABLES}

Table 1 SPC-1553-CAL-MRM gas

Table 2. Molar concentration of the fuel clad pellet

Table 3. MRMF Manifold Volumes

Table 4 - Reduction calculation for all fuel processed since 2014. 
Page intentionally left blank 


\section{ACRONYMS}

\begin{tabular}{|c|c|}
\hline AMU & Atomic mass unit \\
\hline $\mathrm{Ar}$ & Argon gas \\
\hline Atm & atmosphere (pressure) \\
\hline $\mathrm{CO}$ & Carbon Monoxide gas \\
\hline $\mathrm{CO}_{2}$ & Carbon Dioxide gas \\
\hline EG\&G & Edgerton, Germeshausen, and Grier, Inc, the contract administrator for the Mound facility \\
\hline $\mathrm{FC}$ & Fuel Clad \\
\hline GIS & Graphite Impact Shell \\
\hline GPHS & General Purpose Heat Source \\
\hline $\mathrm{He}$ & Helium gas \\
\hline IHS & Isotopic Heat Source \\
\hline INL & Idaho National Laboratory \\
\hline $\mathrm{K}$ & Kelvin \\
\hline mmoles & 1/1000 mole \\
\hline MMRTG & Multi-Mission Radioisotope Thermoelectric Generator \\
\hline MOD & Module \\
\hline MRM & Module Reduction and Monitoring \\
\hline MRMF & Module Reduction and Monitoring Facility \\
\hline $\mathrm{N}_{2}$ & Nitrogen gas \\
\hline $\mathrm{O}_{2}$ & Oxygen gas \\
\hline $\mathrm{O} / \mathrm{Pu}$ & Ratio of oxygen to plutonium in the fuel \\
\hline $\mathrm{Pa}$ & Pascal \\
\hline psia & pounds per square inch absolute \\
\hline $\mathrm{Pu}$ & Plutonium \\
\hline $\mathrm{PuO}_{2}$ & Plutonium Dioxide \\
\hline RGA & Residual Gas Analyzer \\
\hline RPS & Radioisotope Power Systems \\
\hline RTG & Radioisotope Thermoelectric Generator \\
\hline SME & Subject Matter Expert \\
\hline${ }^{\circ} \mathrm{C}$ & Degree Celsius \\
\hline
\end{tabular}


Page intentionally left blank 


\section{Review of the General-Purpose Heat Source Module Reduction and Monitoring Processing at INL}

\section{Introduction}

Early work building General Purpose Heat Source-Radioisotope Thermoelectric Generators (GPHS-RTG) determined that the heat source used for Radioisotope Power Systems (RPS), plutonium dioxide $\left(\mathrm{PuO}_{2}\right)$, needed to be slightly reduced, estimated at $\mathrm{PuO}_{1.98}$, to limit the amount of oxygen inside the generator during ground handling operations.

This work was performed at Mound Laboratory near Dayton Ohio and later transferred to Idaho National Laboratory (INL). The processing of heat sources at Mound and the acceptance specification is described by Ernest Johnson in the paper "Post-encapsulation plutonia reduction for Galileo and Ulysses GPHS modules: The module reduction and monitoring facility (MRMF)" presented at the American Institute of Physics conference in 1991. The heat sources, typically 2 GPHS modules, were loaded into the MRM can with graphite felt used as an insulator, then placed on the Module Reduction Monitoring Facility (MRMF), a vacuum and backfill gas exchange system. The self-heating characteristics of the Pu-238 fuel enabled reduction in an oxygen - carbon reaction at elevated temperatures.

The amount of reduction is dependent on fuel temperature, estimated at $1410 \mathrm{~K}$ by Johnson in "The Behavior of Oxygen Partial Pressure Over Slightly Substoichiometric Plutonia at 1410 K'. This is an important paper in that it describes the basis for the current MRM process. Additionally, Johnson outlines the thermodynamic calculations governing the reduction process and gives a method to determine the maximum graphite temperature using the ratio of $\mathrm{CO}$ and $\mathrm{CO}_{2}$.

Reduction occurs when oxygen from the fuel evolves, exits the iridium cladding through the frit vent, and reacts with the carbon from the graphite of the GPHS module creating $\mathrm{CO}$ and $\mathrm{CO}_{2}$. The ratio of $\mathrm{CO}$ and $\mathrm{CO}_{2}$ is dependent on the maximum graphite temperature of the system assumed to be the floating membrane of the GPHS Graphite Impact Shell (GIS). These gases pass back into the fuel cladding through the frit vent where the $\mathrm{CO}$ reacts with the oxygen disassociated from the fuel, forming $\mathrm{CO}_{2}$. The cyclic reaction continues until near thermodynamic equilibrium is reached, $\sim 1.98$ at a fuel temperature of $1410 \mathrm{~K}$.

According to the post encapsulation paper (Johnson 1991), the gas composition is measured bi-weekly using a Residual Gas Analyzer (RGA) to determine the partial pressure of argon (Ar), helium (He), $\mathrm{CO}_{2}$, and $\mathrm{CO}$. The fuel is considered reduced when the reaction products $\left(\mathrm{CO}\right.$ and $\left.\mathrm{CO}_{2}\right)$ are insignificant, indicated by three consecutive measurements of $\mathrm{CO}$ are below 500 pascals. Each MRM canister is evacuated and backfilled with ultra-high purity argon after every gas composition measurement or gas tap.

In practice, data from 1995 in support of the Cassini mission and IHS-60 processing suggests gas composition measurements (gas taps) were conducted on a weekly basis. In addition, calculations in the EG\&G Mound Applied Technologies "Investigation of Apparent Fuel Clad Pressurization During the MRMF Process", which Ernest Johnson is a co-author, suggest a significantly lower level of reduction than 1.98. The IHS-60 was estimated to be reduced to 1.994 and the Cassini fuel to 1.997 using the Moles of Gas Released columns in Tables 2.1 and 2.2 of the report (Figure 1 and Figure 2). This lower level of reduction is more in line with the mass balance calculations performed at INL, 1.998 for the Mars 2020 mission. 
Table 2.1. 60 Watt MRMF Gas Release Data

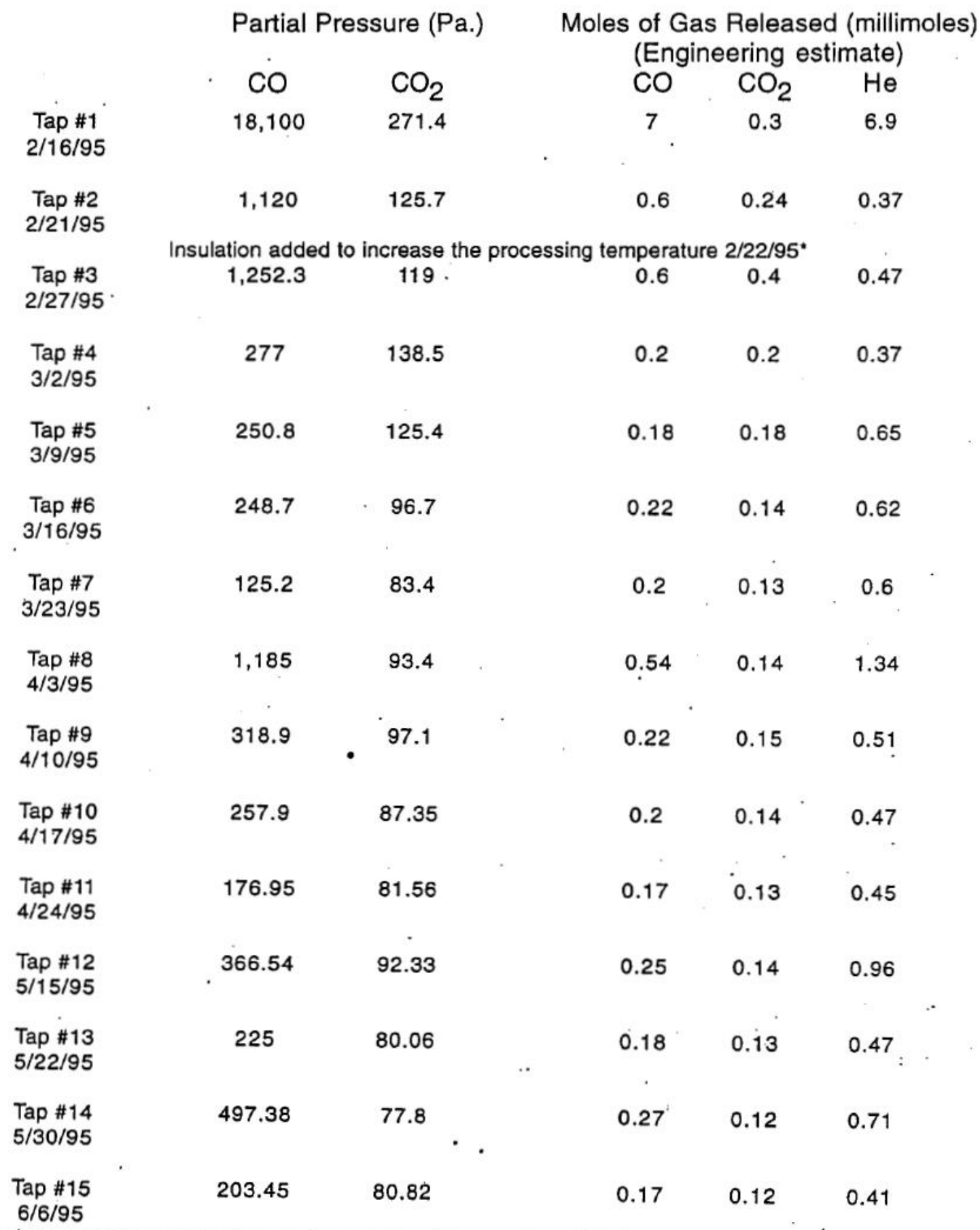

"This increased the temperature on the outside of the can from $204 \mathrm{C}$ to $241 \mathrm{C}$.

Figure 1. Investigation of Apparent Fuel Clad Pressurization IHS-60 MRM data 
Table 2.2. Cassini Production Qual Lot MRMF Gas Release Data

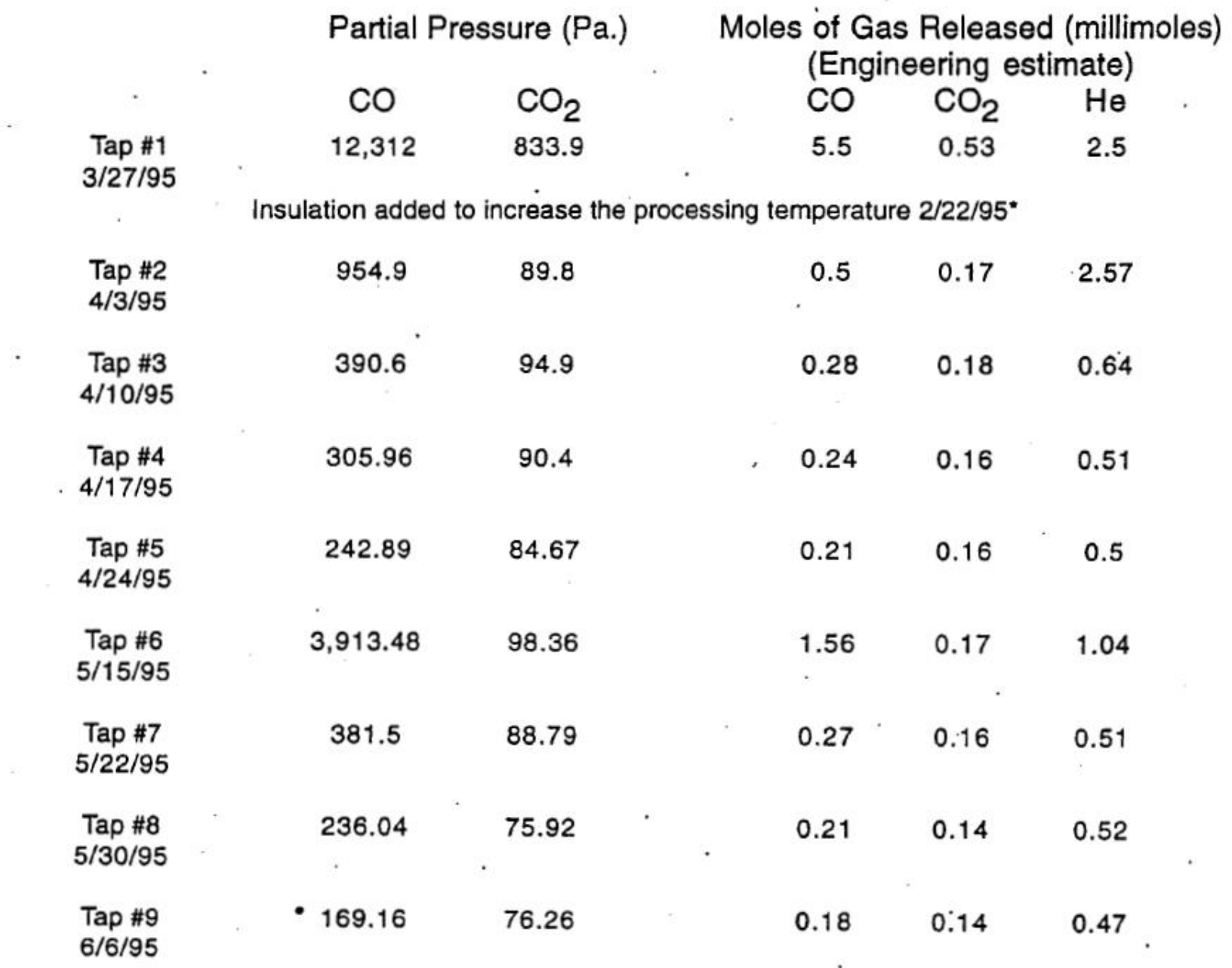

"This increased the temperature on the outside of the can from $194 \mathrm{C}$ to $294 \mathrm{C}$.

Figure 2. Investigation of Apparent Fuel Clad Pressurization Cassini MRM data

\section{MRM Processing}

Module reduction operations start by calibrating the RGA with a gas of known composition. INL uses SPC-1553-CAL-MRM that includes a known amount of Ar, $\mathrm{CO}, \mathrm{CO}_{2}$, and $\mathrm{He}$ (see Table 1). The RGA is calibrated with a consistent gas pressure of $0.50 \pm 0.02$ psia present in the main manifold. The gas is passed from the main manifold to the RGA test chamber through a variable conductance valve to achieve an RGA test pressure of approximately $1 \times 10^{-5}$ torr. The calibration is performed prior to all tests and all tests are performed at the $0.50 \pm 0.02$ psia test pressure. 


\begin{tabular}{|c|c|c|c|c|c|}
\hline $\begin{array}{l}\text { RPS Gas } \\
\text { ID }\end{array}$ & Description & Mixture ga & ses (Concentr & tion) & \\
\hline CAL-MRM & MRM Gas, 86/10/2/2 Ar/CO/He/CO 2 & $\begin{array}{c}\mathrm{Ar} \\
\text { (Balance) }\end{array}$ & $\begin{array}{c}\mathrm{CO} \\
(9.5-10.5 \%)\end{array}$ & $\begin{array}{c}\mathrm{He} \\
(1.9-2.1 \%)\end{array}$ & $\begin{array}{c}\mathrm{CO}_{2} \\
(1.9-2.1 \%)\end{array}$ \\
\hline
\end{tabular}

Cans are loaded, welded closed, backfilled with helium and leak checked prior to MRM processing. Each can has a manual isolation valve that is shut and capped after backfilling. These cans are generally processed in batches and connected to a branch manifold, Manifold A, B or C. Each branch manifold connects to the main manifold isolated by solenoid valves. The cans are connected to a station as determined by the MRM subject Matter Expert (SME). Each station is isolated from the branch manifold using a station solenoid valve.

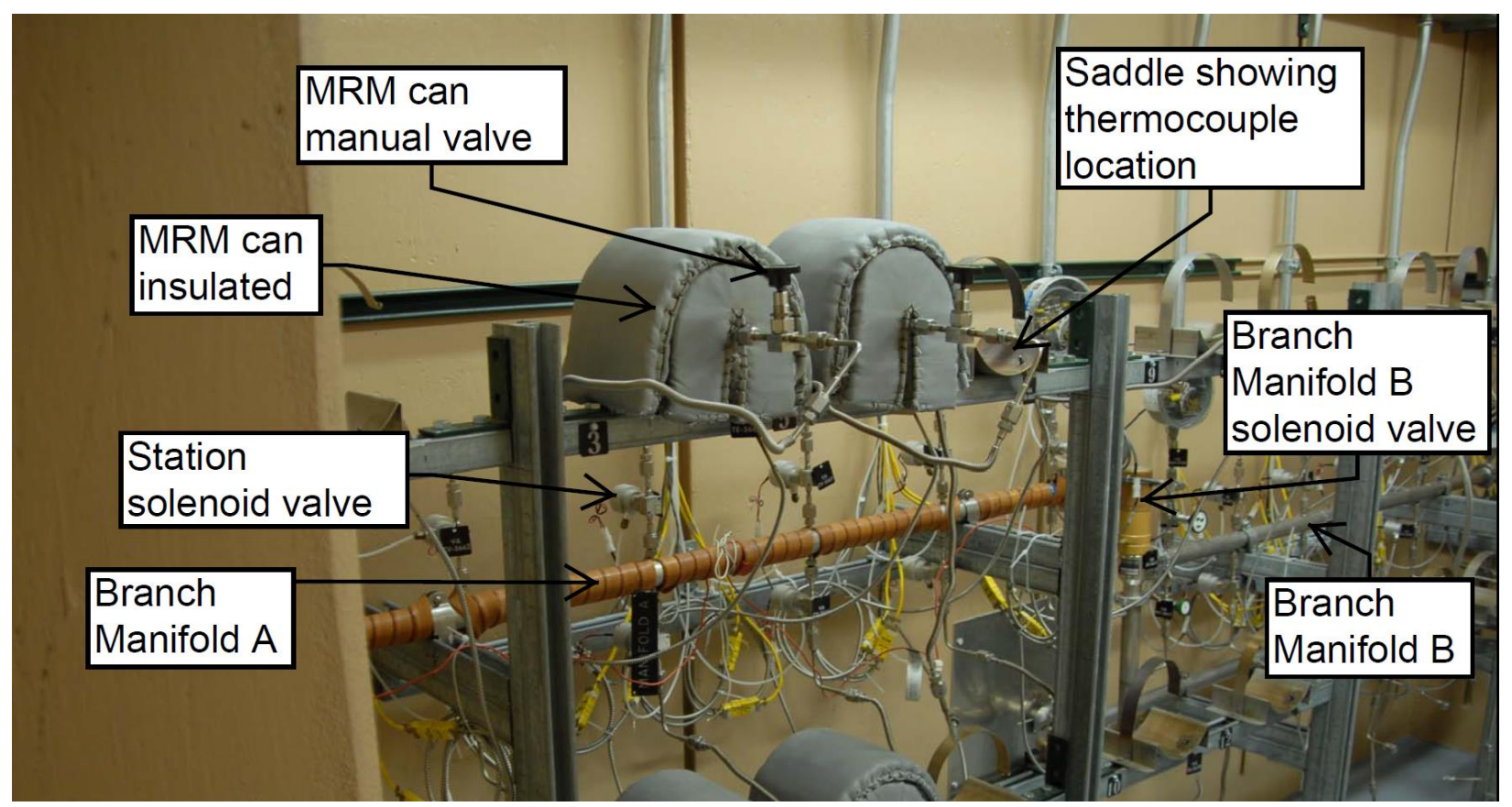

Figure 3. MRMF with multiple cans processing

The system, up to the manual MRM can valve, is evacuated and backfilled with high purity Ar multiple times to ensure minimal impurities are in the system. The manual can valves are opened then the MRM cans are evacuated and backfilled with Ar to 20 psia. The system is configured with the branch manifold solenoid valve closed and the station solenoid valves open to begin processing. Processing always begins with the MRM cans "open to the manifold" where the gas pressure can be monitored. After approximately 24 hours, the cans are insulated to achieve a temperature of 250 to $325^{\circ} \mathrm{C}$ as measured by a thermocouple contacting the side of each can. Inconsistent thermal loading (quantity of fuel) and felt packing methods can result in variable fuel temperatures.

The first gas tap is taken one week into the processing. The RGA is calibrated and the main manifold evacuated. The branch manifold pressure is noted and the manifold solenoid valve is opened to the main manifold. This pressure of the main manifold is recorded as the release pressure on the data sheet for each can. Processing the data has changed over the years. The release pressure was used at Mound and initially at INL when processing the cans closed to the manifold. This will be described in more detail later. The entire system is then pumped down to $0.50 \pm 0.02$ psia as read on the main manifold pressure gauge. An 
RGA data point is taken. The partial pressure of $\mathrm{CO}$ as read on the RGA and the initial branch manifold pressure are used to calculate the partial pressure of $\mathrm{CO}$ in the system. After the gas tap, the system is evacuated, backfilled with Ar and the branch manifold valve shut. The processing continues open to the manifold with weekly gas taps.

CO Partial Pressure Equation

Where

$$
\mathrm{P}_{\mathrm{CO}}=\mathrm{P}_{\operatorname{man}} *\left(\mathrm{P}_{\mathrm{CO} \_ \text {RGA }} / \mathrm{P}_{\mathrm{RGA}}\right) *(101325 / 14.7)
$$

$$
\begin{aligned}
& \mathrm{P}_{\mathrm{CO}}=\text { Partial pressure of } \mathrm{CO}(\mathrm{Pa}) \\
& \mathrm{P}_{\operatorname{man}}=\text { Pressure of the branch manifold prior to release (psia) } \\
& \mathrm{P}_{\mathrm{CO} \_ \text {RGA }}=\text { RGA partial pressure of } \mathrm{CO} \text { (torr) } \\
& \mathrm{P}_{\mathrm{RGA}}=\text { Total pressure of the RGA (torr) } \\
& 101325 \mathrm{~Pa} / \mathrm{atm} / 14.7 \text { psia/atm }=6895(\mathrm{~Pa} / \mathrm{psia})
\end{aligned}
$$

Most of the gas is evolved from the fuel after several gas taps. At this point, the SME will direct the station solenoid valve shut to begin processing "closed to the manifold". The pressure inside the can is not known and must be estimated during the $\mathrm{CO}$ partial pressure calculation. Each can is evacuated and backfilled individually to 20 psia and the station solenoid valve is shut and leak tested. The branch manifold pressure is also set to 20 psia to minimize chance gas exchange with the MRM cans.

Gas taps performed with the cans closed to the manifold are slightly different. Both the branch manifold and main manifold are evacuated. After the branch manifold solenoid valve is shut, the station solenoid valve is opened to release the MRM gas into just the branch manifold. Historically, this release pressure was not recorded, but this changed during processing of the Mars 2020 fuel. The branch manifold gas is then released to the main manifold where this release pressure is recorded. The system is pumped to $0.50 \pm 0.02$ psia and the RGA sample taken. The partial pressure of $\mathrm{CO}$ as read on the RGA and the estimated MRM can pressure is used to calculate the partial pressure of CO in the MRM can. Cans are processed until each one generates less than $500 \mathrm{~Pa}$ of $\mathrm{CO}$ for three consecutive taps.

$$
\mathrm{P}_{\mathrm{CO}}=\mathrm{P}_{\text {can }} *\left(\mathrm{P}_{\mathrm{CO} \_ \text {RGA }} / \mathrm{P}_{\mathrm{RGA}}\right) * 6895
$$

Where

$$
\mathrm{P}_{\mathrm{can}}=\text { Estimated pressure of the MRM can (psia) }
$$

Mound document MD-21785, Issue 18, the latest known version of the Module Reduction and Monitoring Facility Operations instructions was reviewed to determine if any differences between processing at Mound vs INL could be identified. The procedures appear consistent including using the same equipment. The only difference noted is that INL processes the MRM can with a surface temperature between 250 and $325^{\circ} \mathrm{C}$. The can temperature is not defined in the Mound instructions. Data from the investigation of the IHS-60 apparent pressurization (Merten, et all 1995) shows processing IHS60 cans at $241^{\circ} \mathrm{C}$ and Cassini cans at $294^{\circ} \mathrm{C}$. It is not known when the temperature range was imposed.

A potential difference in the processing is the packaging of the fuel in the can. The fuel is packaged in the can surrounded by pieces of graphite felt which act as a thermal insulator. The configuration of the felt is not controlled and therefore open to process variation. When the process was transferred to INL, Mound personnel were hired by INL to assure a smooth transition of the method and it is reasonable to assume the same packaging techniques were used. INL personnel span the original Pluto New Horizon GPHSRTG build to the latest MMRTG build. 


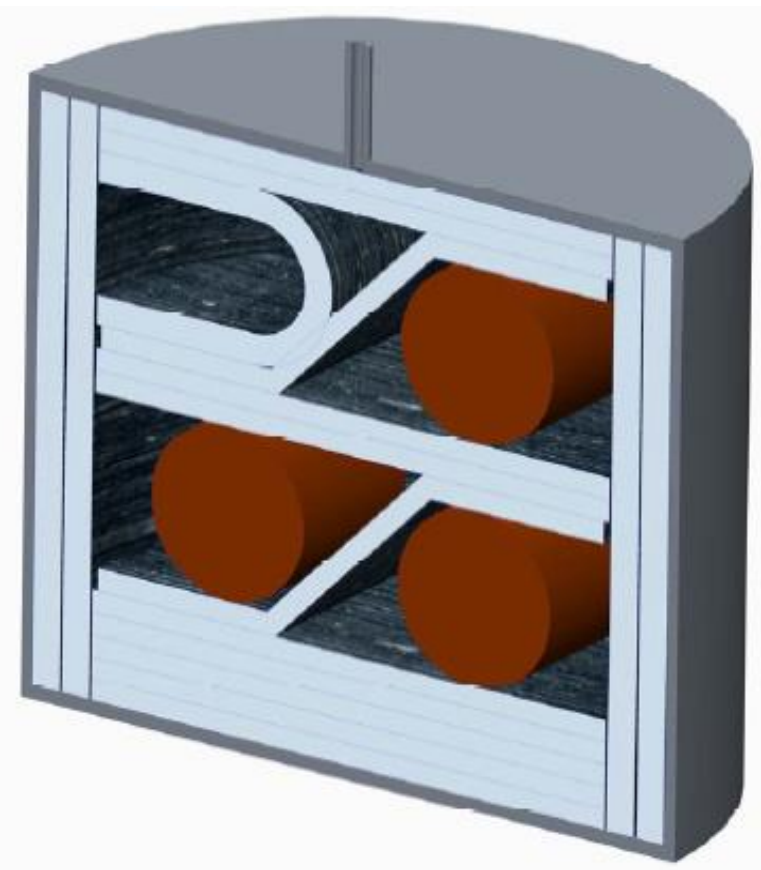

Figure 4. Packaging drawing of MRM can L134

A change to packaging configuration occurred in April 2017. The graphite packing methodology was changed from a random stuffing of felt (Figure 4) to felt disks stacked to fill more of the MRM can volume. Cylinder cutouts were provided to suspend each GIS in the approximate center of the MRM can (Figure 5). Historically, assembled modules (2 GIS installed in an aeroshell) were processed, but the IHS60 heat sources were processed as 3 GIS in one MRM can. Afterwards, the IHS-60 heat sources were removed from the GIS and welded into the heat source form. At INL, GIS were loaded into MRM cans at first using the random felt method, then changed to the stacked disks method.

A second potential difference in processing at INL and Mound is the purity of the gas in the can after completing a gas tap. General use gas bottle regulators from Mound were transferred to INL as part of the MRM process equipment. Slowly, these regulators have been replaced with research grade regulators designed to minimize impurities. Processing MRM cans leaves some room for interpretation of gas purity. A typical evacuation step will lead to evacuate to less than 50 mtorr as measured by the main manifold thermocouple gauge. It is typical to pump the system down lower than the thermocouple gauge can measure. It is not known what effect this has on the pressure inside an MRM can where the can is evacuated through a small diameter tube or the effects of the gas left in the clads that have to pass through the frit vent. It is possible the impurities remaining in the can using present evacuation techniques are different than when processed at Mound. 


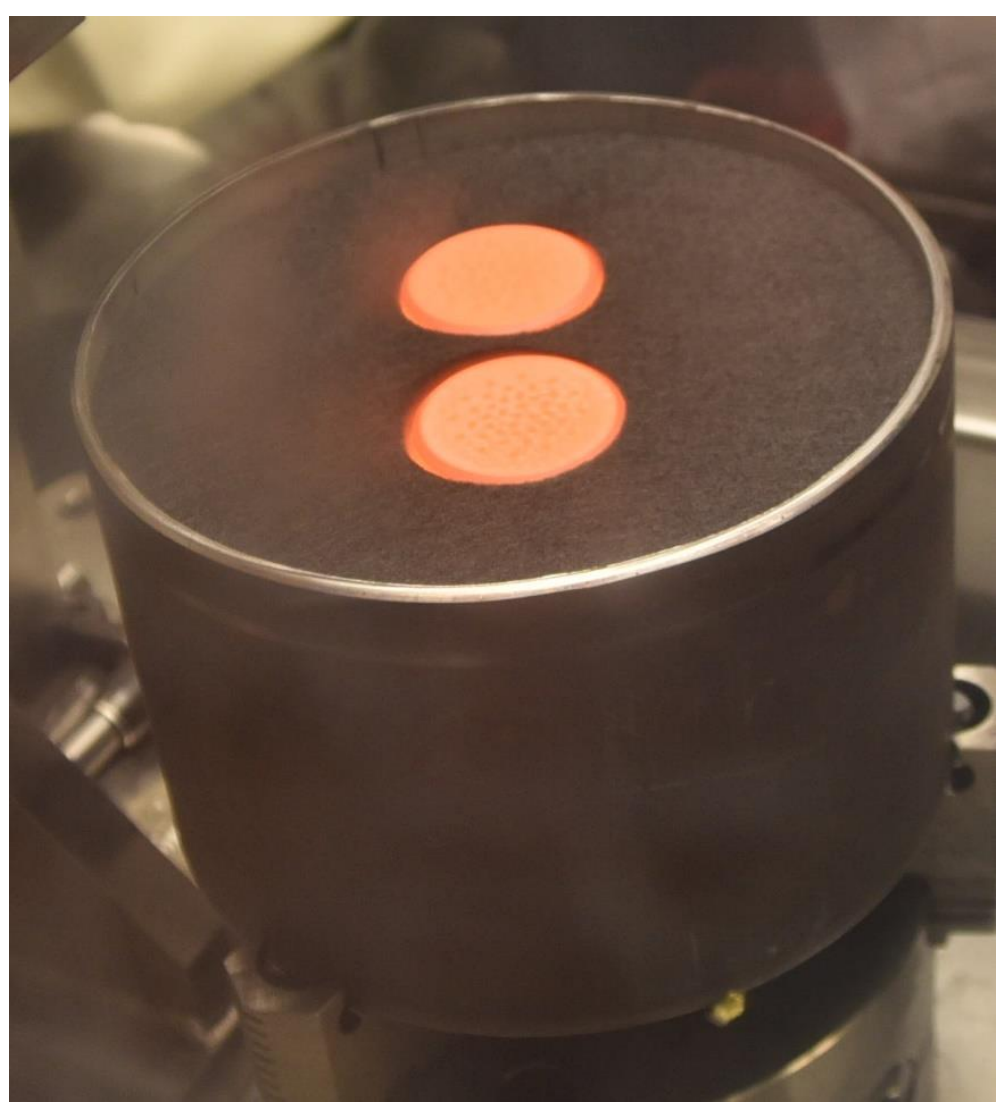

Figure 5. Updated GIS packaging with 2 GIS post can cutting

\section{Estimating Can Pressures when Closed to the Manifold}

Estimating the MRM can pressure during processing closed to the manifold has been performed differently over the years. The difficulty arises due to many factors: the fuel is self-heating, making it difficult to apply the ideal gas law, the gas temperature is not known, the MRM can volume is variable due to, fuel load, felt packing, weld shrinkage and variability in the station flex lines. Mound developed a method of testing the "apparent volume of can" by backfilling the can to a set pressure, allowing the system to become thermally stable, evacuating the manifold then dumping the MRM can gas into the manifold with known volume.

$$
\mathrm{V}_{\text {app }}=\mathrm{P}_{\text {rel }} * \mathrm{~V}_{\text {man }} /\left(\mathrm{P}_{\mathrm{O}}-\mathrm{P}_{\text {rel }}\right)
$$

Where

$$
\begin{aligned}
& \mathrm{V}_{\text {app }}=\text { Apparent volume of the MRM can (L) } \\
& \mathrm{P}_{\text {rel }}=\text { Release pressure of the manifold and MRM can (psia) } \\
& \mathrm{V}_{\operatorname{man}}=\text { Volume of the manifold }(\mathrm{L}) \\
& \mathrm{P}_{\mathrm{O}}=\text { Initial pressure in the MRM can (psia) }
\end{aligned}
$$

The intent at INL was to perform the calculation as done at Mound. There appears to have been a mistake in interpreting the apparent volume that resulted in a miscalculation of the $\mathrm{CO}$ partial pressure. This is documented in the Craig Dees memo dated June 19, 2013 in support of Problem/Failure Review (P/FR) form INL-IP-FY13-913. This memo concluded that calculating the gas pressure was less accurate than using the backfill gas pressure performed on the previous tap. This may not be accurate in the initial gas 
taps where a significant amount of helium and oxygen are evolved from the fuel, but is a reasonable assumption during the final 3 gas taps where the $\mathrm{CO}$ partial pressure is less than $500 \mathrm{~Pa}$.

Another method of estimating the gas pressure is to use the release pressures of the can into two other known volumes. This was developed and performed during the recent servicing of legacy heat sources at INL. The MRM output has significant noise requiring averaging several data points and has only been used processing the data in this report. The gas pressure, the number of moles of gas in the MRM can, and the variable equal to the average MRM can gas temperature divided by the MRM can volume is determined (see Appendix A for more details). The recent change in recording the release pressures of the can into the branch manifold and the branch manifold / can volume into the main manifold has opened different methods of analyzing the RGA data that will be utilized in subsequent mass balance calculations.

\section{Addition Comments to the Published Literature}

Mass balance calculations presented a reduction of 1.9931 (Johnson 1982). Merten et al. (1995) reported data suggesting the IHS-60 fuel was reduced to 1.995 and the Cassini fuel to 1.997. A reduction to 1.997 is consistent with the GPHS F2, F6 and F7 units reported in Carbon Monoxide Levels in Cassini RTGs (GESP 7253 1997) where 125.86, 112.17 and 119.74 mmoles of oxygen were removed during MRM processing. This level of reduction is repeated in the 1991 Johnson paper, yet there continues to be a declaration that the fuel is being reduced to $\sim 1.98$, which seems to contradict the data.

\section{Amount of Plutonia per Fuel Clad}

The amount of plutonia present in each fuel clad needs to be determined prior to calculating the reduction of fuel using a mass balance technique. The Los Alamos document LA-UR-00-4157, "Fabrication of Radioisotope Heat Sources for Space Missions" gives a typical chemical composition of a fueled clad. Johnson's 1982 paper used 4.63 moles per MRM can containing 2 GPHS modules. The calculation in Table 2 yields 4.47 moles per MRM can containing two modules. The mass balance calculations performed subsequently use the data from Table 2 .

Table 2. Molar concentration of the fuel clad pellet

\begin{tabular}{|l|l|l|l|l|}
\hline Isotope & $\begin{array}{l}\mathrm{PuO}_{2} \\
\text { molecular } \\
\text { weight }\end{array}$ & $\begin{array}{l}\text { Composition } \\
\text { percentage }\end{array}$ & $\begin{array}{l}\text { Mass assuming } \\
151.1 \mathrm{~g} \text { pellet } \\
\text { weight }\end{array}$ & $\begin{array}{l}\text { Moles per } \\
\text { pellet }\end{array}$ \\
\hline${ }^{238} \mathrm{Pu}$ & 270 & 83.5 & 126.2 & 0.467 \\
\hline${ }^{239} \mathrm{Pu}$ & 271 & 14.01 & 21.2 & 0.078 \\
\hline${ }^{240} \mathrm{Pu}$ & 272 & 1.98 & 3.0 & 0.011 \\
\hline${ }^{241} \mathrm{Pu}$ & 273 & 0.37 & 0.6 & 0.002 \\
\hline${ }^{242} \mathrm{Pu}$ & 274 & 0.14 & 0.2 & 0.001 \\
\hline & & Total (8 pellets) & $4.474 \mathrm{~mol}$ \\
\hline
\end{tabular}

\section{$\mathrm{CO}_{2}$ / CO Measurements}

The RGA partial pressure of $\mathrm{CO}_{2}$ is below the limit of detection during most of the processing at INL. It is not obvious to the user that this value is limited due to the calibration factors used. The displayed partial pressure indicates a reading of $10^{-9}$ torr or higher, which is higher than the oxygen partial pressure of $10^{-10}$ torr. However, the calibration factor for $\mathrm{CO}_{2}$, as low as 3.4 to as high as 19 , is multiplied by the RGA reading resulting in a reported value higher than the minimum detectable pressure.

There needs to be a detectable amount of $\mathrm{CO}_{2}$ and therefore a significant amount of $\mathrm{CO}$ to obtain an estimate of the maximum carbon temperature. A note in the 1982 paper at the bottom of Table 4 suggest 
$\mathrm{CO}_{2}$ is not detectable. It appears Mound was also attempting to measure below the detectability of $\mathrm{CO}_{2}$ with the same instrumentation transferred to INL. Because of this, the calculated maximum carbon temperature appears to decrease with time. There appears to be a limited number of test points in which this method yielded a reasonable maximum carbon temperature. The initial gas tap with multiple cans open to the manifold typically yielded the highest carbon temperature.

$\mathrm{CO}$ and nitrogen $\left(\mathrm{N}_{2}\right)$ have the same atomic mass units (AMU) of 28 and therefore, one cannot distinguish between the two gases by looking at the primary peaks. Each gas molecule cracks into molecules or in this case individual atoms at a predictable rate and shows up as smaller peaks at different AMUs. CO cracks into a single carbon atom (12 AMU) and a single oxygen atom (16 AMU). These atoms are secondary peaks for other gases in the system and do not give much information. Nitrogen breaks into two individual nitrogen atoms (14 AMU) $7 \%$ of the time for a $14 \%$ relative intensity as stated on the Dycor ${ }^{\circledR}$ Mass Spectra Fragmentation Pattern Calculator. It is current practice to scan AMU 14 as a diagnostic tool to help determine if air is leaking into the system. Air would also allow $\mathrm{O}_{2}$ into the system creating $\mathrm{CO}$. A quick check to determine if air is leaking into the system is to multiply the AMU 14 partial pressure by $7(100 \% / 14 \%)$ and verify the value is significantly below the AMU 28 pressure.

\section{RGA Calibration Factors}

During RGA calibration, the RGA total pressure is scaled to meet the total pressure of the calibrated ion gauge. The individual AMU for each gas species is scaled until the RGA pressures are approximately equal to the theoretical RGA pressures for the specific partial pressure of the calibration gas. The total RGA pressure is not a summation of the partial pressures, it is an independent measurement designed to protect the RGA from operating at too high a pressure and damaging the instrument. Figure 6 demonstrates that the calibration factors trend well with the other gas species and that the ratio of the $\mathrm{CO}$ and $\mathrm{Ar}$ is reasonably constant at approximately 1. 


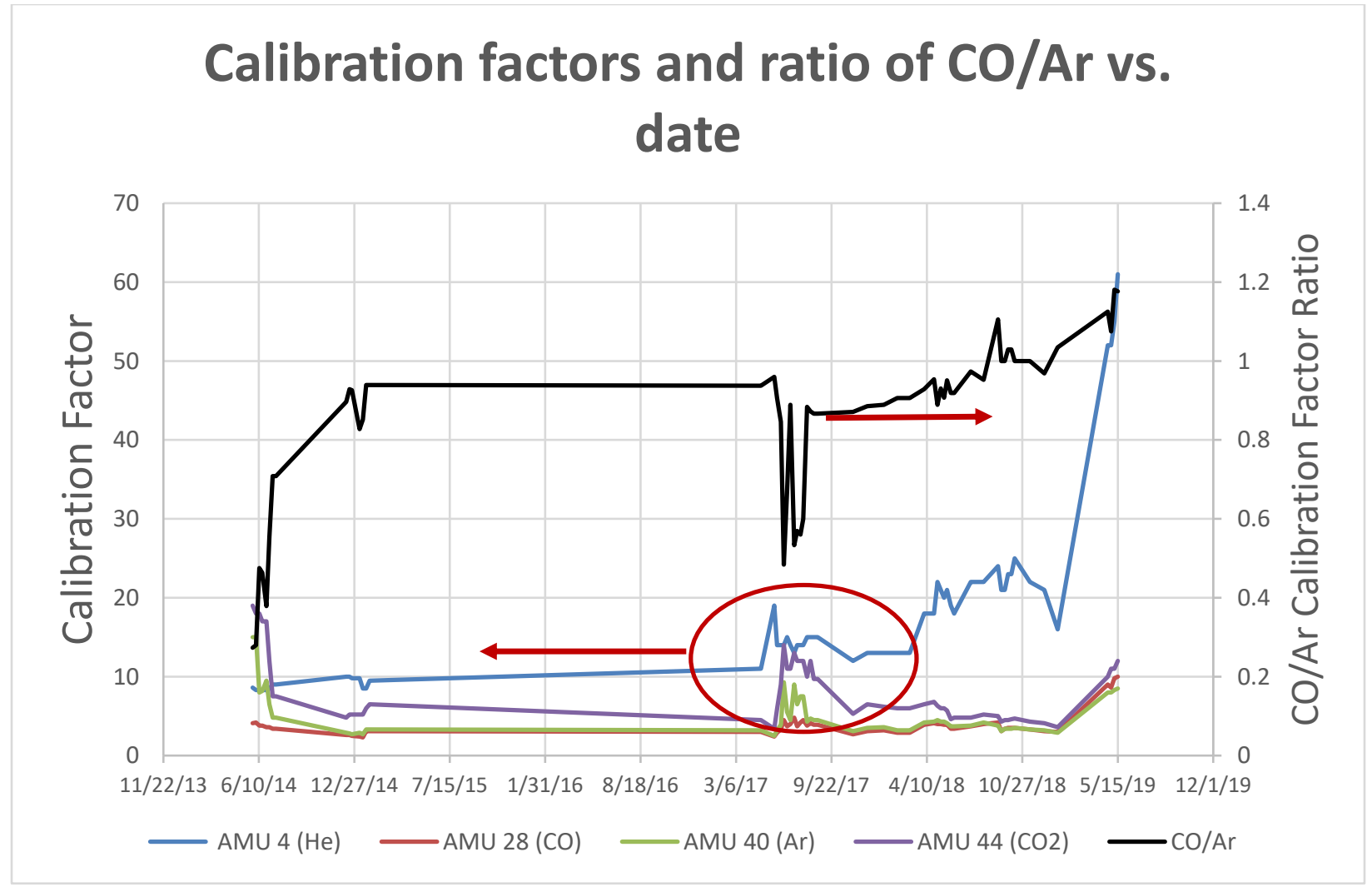

Figure 6 - Calibration factors and ratio of $\mathrm{CO} /$ Ar vs time

\section{Mass Balance Calculations}

The mass balance calculation is a straightforward technique that measures the oxygen in the MRM gas composition and removes that value from the calculated oxygen remaining in the fuel assuming the initial stoichiometry of the fuel is $\mathrm{PuO}_{2}$. The partial pressure of $\mathrm{CO}$ and $\mathrm{CO}_{2}$ are calculated and the number of moles of the gases are estimated. Finally, the oxygen is subtracted from the number of moles of $\mathrm{PuO}_{2}$.

Three different methods of calculating the number of moles of $\mathrm{CO}$ and $\mathrm{CO}_{2}$ are presented. The primary difference between these methods is how the mole fractions are calculated. The first method aligns with the operating procedure. This method ratios the RGA $\mathrm{CO} / \mathrm{CO}_{2}$ partial pressure with the overall RGA pressure. As stated above, the overall RGA pressure is independent of the partial pressures of gases present, a design feature to protect the RGA from damage. The second method eliminates this error by using a summation of the calibration gas partial pressures. Analog RGA measurements taken during gas taps indicates the only gases present at significant values are the calibration gases, $\mathrm{Ar}, \mathrm{He}, \mathrm{CO}$ and $\mathrm{CO}_{2}$. This is a more accurate comparison to the individual gas partial pressures. Both methods ignore the evolution of $\mathrm{He}$ and the production of reactant gases $\left(\mathrm{CO}\right.$ and $\left.\mathrm{CO}_{2}\right)$ because these methods assume the fill pressure is the only pressure in the can.

The third method to calculate $\mathrm{CO} / \mathrm{CO}_{2}$ ratio eliminates the error of the evolution of gases from the fuel by using the RGA partial pressure of Ar as the denominator. The can is filled with pure Ar to a set pressure. $\mathrm{Ar}$ is neither consumed nor evolved from the fuel and is therefore present at a constant 20 psia throughout processing. The same instrument is used to measure $\mathrm{CO} / \mathrm{CO}_{2}$ and $\mathrm{Ar}$ and all are calibrated with the same gas at the same time. In almost all cases presented, this method yields the highest $\mathrm{CO} / \mathrm{CO}_{2}$ pressures and therefore the most conservative result, from a processing standpoint. 
Using the ideal gas law, the number of moles can be calculated provided the volume is known. The difficulty comes from estimating the apparent volume of the MRM can. This is estimated using the two known volume pressure releases described in Appendix A. This provides a total estimate of moles of gas in the can as well as the temperature to volume (T/V) ratio variable. To minimize the can volume and can temperature error, the reduction calculation uses the measured release pressure to calculate an average $\mathrm{T} / \mathrm{V}$ ratio over several test points. The release pressure and the volumes of the manifolds at room temperature are used to estimate the number of moles in the MRM system. The release pressure and the $\mathrm{T} / \mathrm{V}$ ratio are used to calculate the moles remaining in the can. The summation of these two values equals the total number of moles in the system. Finally, as a quality check, the numbers of moles calculated during the two known volume releases and the calculated moles of gas in the MRM can and manifolds at release are compared. The results match within $10 \%$ in all cases except for cans processed on the B manifold, which are within $20 \%$.

$$
N_{t o t}=\frac{P_{r e l}}{R} *\left(\frac{1}{\left(\frac{T}{V}\right)_{1}}+\frac{1}{\left(\frac{T}{V}\right)_{2}} \ldots+\frac{V_{m a n}}{T_{a m b}}\right)
$$

Where

$\mathrm{N}_{\text {tot }}=$ Total number of moles of gas presen

$\mathrm{P}_{\mathrm{rel}}=$ Final release pressure $(\mathrm{Pa})$

$\mathrm{R}=$ Ideal gas constant $(\mathrm{J} /(\mathrm{Mol} * \mathrm{~K})$

$(\mathrm{T} / \mathrm{V})_{1}=$ The average temperature volume variable calculated from release pressures $\left(\mathrm{K} / \mathrm{m}^{3}\right)$ for Can 1 and additional cans when processed open to the manifold and only one can when closed to the manifold.

$\mathrm{V}_{\text {man }}=$ Volume of the manifold $\left(\mathrm{m}^{3}\right)$

$\mathrm{T}_{\mathrm{amb}}=$ Ambient temperature $(298 \mathrm{~K})$

And

$$
\begin{gathered}
N_{C O}=N_{t o t} *\left(\frac{P_{C O}}{P_{R G A}}\right) \quad \text { Or } \quad N_{C O}=N_{t o t} *\left(\frac{P_{C O}}{P_{S U M}}\right) \\
N_{C O 2}=N_{t o t} *\left(\frac{P_{C O 2}}{P_{R G A}}\right) \quad \text { Or } \quad N_{C O 2}=N_{t o t} *\left(\frac{P_{C O 2}}{P_{S U M}}\right) \\
O / P u=\left(2 * N_{P u}-\Sigma\left(N_{C O}+2 * N_{C O 2}\right)\right) / N_{P u}
\end{gathered}
$$

Where

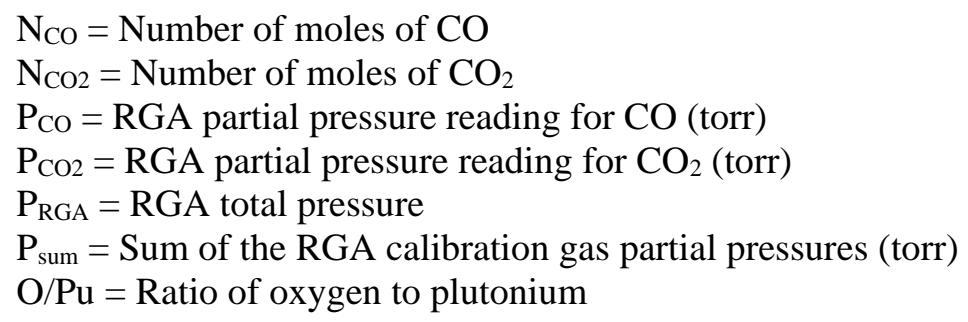

Reduction data from MRM can L1303 was used to calculate the number of moles of gas remaining in the can compared to the total number of moles in the manifold / can system. This can was installed on manifold A without other cans. When processed open to the manifold, only $36 \%$ of the gas in the system 
remained in the can. When processed closed to the manifold, only $12 \%$ of the gas remained in the can. This changed to $13 \%$ when the can was moved to storage on manifold $\mathrm{C}$. The can was processed open to the manifold for 3 weeks where the can reduced from 2.0 to 1.997 . The total reduction for this can was to 1.991. Therefore, most of the reduction that occurred was closed to the manifold where $88 \%$ of the gas was in the known volume of the manifolds, at ambient temperature.

The Ar partial pressure calculation relied on the original $138 \mathrm{kPa}(20 \mathrm{psia})$ of Ar loaded into the system and the ratios of the RGA partial pressures of each gas to the partial pressure of Ar. In this case, the volume of the can is not known, so the average T/V value was utilized. Each gas species pressure was calculated and then utilized for the calculation of the number of moles. This method accounts for the additional gases released or generated when processing.

$$
P_{\mathrm{CO} / \mathrm{CO} 2}=138 \mathrm{kPa} *\left(\frac{P_{\mathrm{CO} / \mathrm{CO} 2}}{P_{A r}}\right)
$$

And

$$
N_{\mathrm{CO} / \mathrm{CO} 2}=\frac{P_{\mathrm{CO} / \mathrm{CO} 2}}{R} *\left(\frac{1}{\left(\frac{T}{V}\right)_{1}}\right)
$$

There is an error in the T/V variable, in that the calculated pressure of the can using the Appendix A method divided by the sum of the gas partial pressures averages $88 \%$ on cans processed on manifolds $\mathrm{A}$ and C. Cans processed on manifold B averaged 140\%. Evaluation of the branch manifold pressure transducer readings to the main manifold transducer suggests the manifold B pressure transducer pressure reading was not accurate. In addition, this method assumes Ar is not added or removed. This is not the case for cans where pressure checks were performed. These cans generated significant $\mathrm{CO}$ pressures during this time, so Ar was likely vented as the manual valve was cycled.

\section{Assumptions}

Processing the can open to the manifold exposes several heat sources to the same volume. The amount of oxygen removed is assumed to be evenly distributed even if the heat sources have different can temperatures.

Manifold volumes in Table 3 are based on the Munns memo titled "MRM Can Internal Volume Determination", dated May 15, 2005.

Table 3. MRMF Manifold Volumes

\begin{tabular}{|l|l|}
\hline Manifold & Volume (L) \\
\hline A & 1.79 \\
\hline B & 1.87 \\
\hline C & 2.27 \\
\hline Main & 5.05 \\
\hline
\end{tabular}

\section{Typical Data Sheet}

A marked-up copy of the April 27, 2017 RGA scan of can L1308 is shown in Figure 7. This gas tap yielded a high partial pressure of $\mathrm{CO}$ that was measured after prolonged storage at a can temperature 100 ${ }^{\circ} \mathrm{C}$ cooler than during processing. The printout shows the masses of the constituent gasses, the calibration factors, and the partial pressures of each gas. The secondary peak of nitrogen $(\mathrm{N})$ is two orders of 
magnitude low, indicating that $\mathrm{N}$ is not the cause of the high $\mathrm{CO}$ interpretation. The $\mathrm{CO}_{2}$ value when adjusted for the calibration factor shows a value equivalent to oxygen, which is undetectable. Previous records indicate the can was filled to 20.23 psia during the last processing cycle. The partial pressures using different molar ratios are displayed showing 4 to $7 \mathrm{kPa}$ of $\mathrm{CO}$ present. 


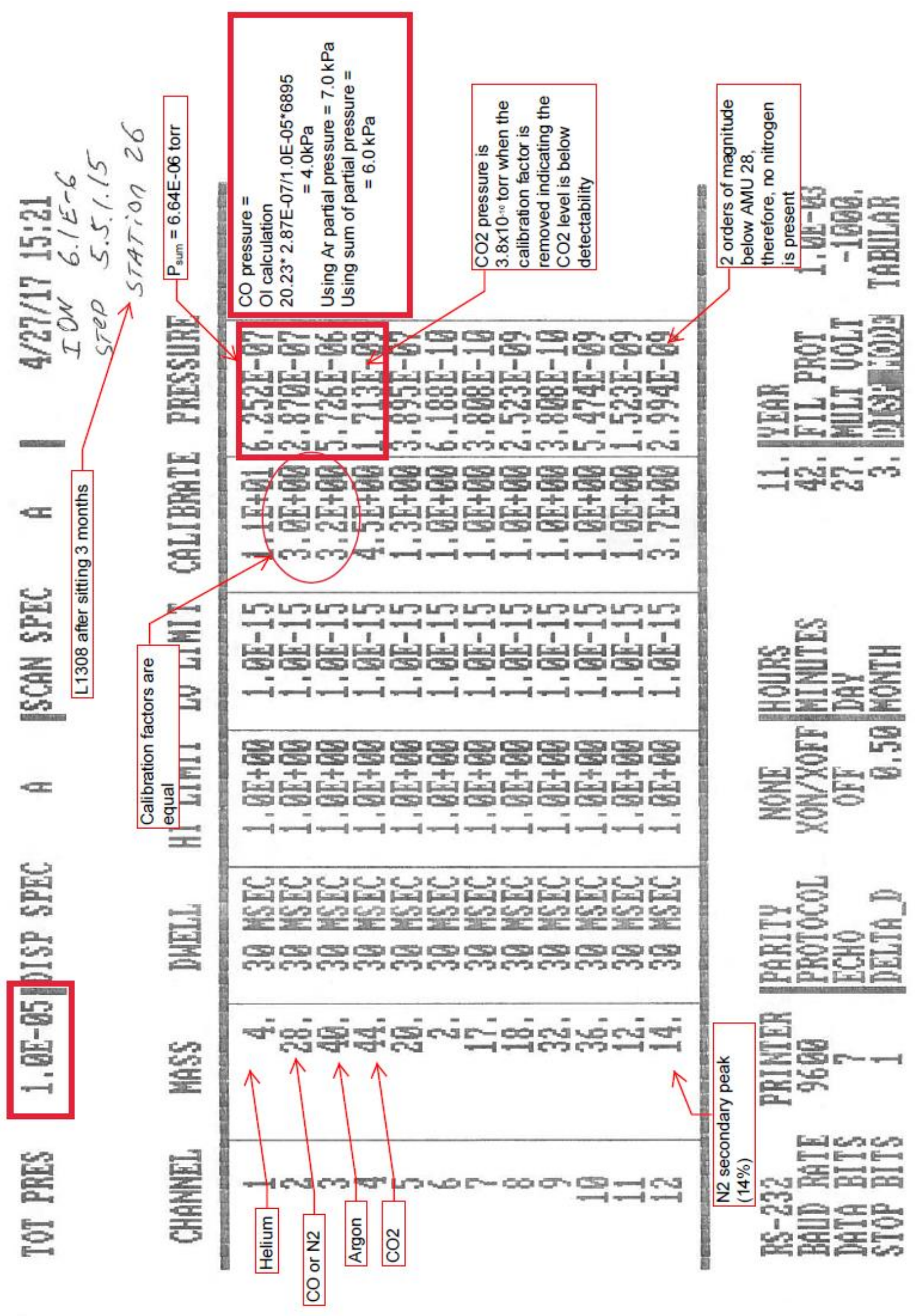

Figure 7 - April 27, 2107 RGA scan of can L1308 


\section{Results}

The reduction calculation for all fuel processed since 2014 is shown in Table 4. Only one can, L1303, saw a significant reduction, due mostly to the large reaction products that occurred over a 74 day inactivity period. Reduction levels are consistent with the mass balance calculations performed at Mound but are an order of magnitude less than the 1.98 suggested in early papers.

Figure 8 through Figure 20 show the can histories and reduction at each gas tap. The left axis is the $\mathrm{CO}$ pressure calculated using the three different methods described above. The value of this axis varies for each can processed. The right axis is the calculated $\mathrm{O} / \mathrm{Pu}$ value typically ranging from 1.98 to 2.0 . The exception is the flight modules where little additional reduction was detected above the GIS processing. Five of the thirteen cans show a tendency for additional reduction after the processing was declared complete. These five cans are the earliest cans reviewed.

Using the ratio of $\mathrm{P}_{\mathrm{CO}} / \mathrm{P}_{\mathrm{Ar}}$ and $138 \mathrm{kPa}$ fill pressure to calculate the partial pressure of $\mathrm{CO}$ is the most accurate and easiest method when performing weekly and monthly gas taps. The $\mathrm{CO}$ pressure reduces to $138 \mathrm{kPa} *\left(\mathrm{P}_{\mathrm{CO}} / \mathrm{P}_{\mathrm{Ar}}\right)$ and can be accomplished using the upgraded MRM system RGA output. This method is not accurate when pressure checks that do not maintain gas isolation are involved since the partial pressure of Ar is no longer known.

More information could be obtained during this process. An electron multiplier on the RGA may allow the $\mathrm{CO}_{2}$ partial pressure to be measured, thus allowing for a reliable maximum carbon temperature calculation. This maximum temperature is located at the floating membrane and close to the hot operating temperature of the fueled clad. Cans are now stored on the MRM system for years and understanding the clad temperature may be beneficial. Until the 2017 felt packing change, there was no real method of calculating the clad temperature analytically due to the variability of the packing. Controlling the felt configuration and minimizing gaps opens the ability to thermally model the can. Having a reasonable estimate of the floating membrane temperature allows independent verification of the model. In addition, helium is evolving from the fuel during processing. Known fuel temperatures could strengthen helium release models used by RPS providers.

There have been several occasions where processing was deemed complete followed by excessively high levels of CO after periods of inactivity at both Mound and INL.

1. IHS-60 Tap \#8 on 4/3/95 (Figure 1). This is an intriguing event in that the period of inactivity is only 11 days. In the four weeks prior to the 11-day period, the CO pressure measured 277, 251, 249 and $125 \mathrm{~Pa}$. If we assume $250 \mathrm{~Pa}$ of pressure build over 7 days and project that to 11 days, the CO pressure should have measured $393 \mathrm{~Pa}$. There was $1185 \mathrm{~Pa}$ measured on day 11 . He evolution was $130 \%$ the projected rate.

2. Cassini Tap \#6 on 5/15/95 (Figure 2). This event had a period of inactivity of 21 days. Taps $1-5$ measured 12,313, 955, 390, 306 and 243Pa. If one assumes $400 \mathrm{~Pa}$ weekly gas pressure projected to 21 days yields $1200 \mathrm{~Pa}$ of CO. Taps \#6 was $3913 \mathrm{~Pa}$. He evolution was $66 \%$ the projected rate

3. INL Can L1303 tap\# 10 on 11/6/17 (Figure 14). The previous 4 weekly taps measured 156 to 160 $\mathrm{Pa}$ of CO. At $160 \mathrm{~Pa}$ per week projected over 74 days yields $1691 \mathrm{~Pa}$ where 32,812 $\mathrm{Pa}$ was measured (using the RGA partial pressure of Ar). He evolution was 100\% the projected rate.

4. Figure 10 through Figure 13 also shows this behavior, but the extended period of monthly gas checks prevents detailed analysis. However, the plots show an excessively high value considering the can storage temperature is $100^{\circ} \mathrm{C}$ lower than the processing temperature. Two of these cans also show a rise in $\mathrm{CO}$ over the same 74-day period of can L1303. 
Table 4 - Reduction calculation for all fuel processed since 2014.

\begin{tabular}{|c|c|c|c|c|c|c|}
\hline \multirow{2}{*}{$\begin{array}{l}\text { MRM } \\
\text { Can }\end{array}$} & \multirow[t]{2}{*}{ Contents } & \multirow{2}{*}{$\begin{array}{l}\text { GIS/MOD } \\
\text { ID }\end{array}$} & \multirow[t]{2}{*}{ FC ID } & \multicolumn{3}{|c|}{ Reduction Method } \\
\hline & & & & $\begin{array}{l}\text { RGA Total } \\
\text { pressure }\end{array}$ & $\begin{array}{c}\text { Sum of } \\
\text { Partial } \\
\text { Pressures }\end{array}$ & $\begin{array}{l}\text { Argon } \\
\text { pressure }\end{array}$ \\
\hline L126 & 3 GIS & $\begin{array}{l}3173 \\
3174 \\
3180\end{array}$ & $\begin{array}{l}\text { FC0455 / FC0459 } \\
\text { FC0446 / FC0454 } \\
\text { FC0452 / FC0453 }\end{array}$ & 1.998 & 1.997 & 1.997 \\
\hline L134 & 3 GIS & $\begin{array}{l}3175 \\
3177 \\
3179\end{array}$ & $\begin{array}{l}\text { FC0448 / FC0442 } \\
\text { FC0441 / FC0457 } \\
\text { FC0449 / FC0451 }\end{array}$ & 1.998 & 1.997 & 1.997 \\
\hline L1301 & 3 GIS & $\begin{array}{l}\text { F3219 } \\
\text { F3213 } \\
\text { F3214 }\end{array}$ & $\begin{array}{l}\text { FC0494 / FC0503 } \\
\text { FC0501 / FC0504 } \\
\text { FC0498 / FC0505 }\end{array}$ & 1.999 & 1.999 & 1.999 \\
\hline L1303 & 2 GIS & $\begin{array}{l}\text { F3211 } \\
\text { F3212 }\end{array}$ & $\begin{array}{l}\text { FC0478 / FC0477 } \\
\text { FC0479 / FC0480 }\end{array}$ & 1.995 & 1.991 & 1.990 \\
\hline L1306 & 2 GIS & $\begin{array}{l}\text { F3215 } \\
\text { F3216 }\end{array}$ & $\begin{array}{l}\text { FC0496 / FC0499 } \\
\text { FC0497 / FC0502 }\end{array}$ & 1.999 & 1.999 & 1.999 \\
\hline L1307 & 3 GIS & $\begin{array}{l}\text { F3208 } \\
\text { F3209 } \\
\text { F3210 }\end{array}$ & $\begin{array}{l}\text { FC0461 / FC0466 } \\
\text { FC0464 / FC0465 } \\
\text { FC0471 / FC0467 }\end{array}$ & 1.999 & 1.998 & 1.998 \\
\hline L1308 & 3 GIS & $\begin{array}{l}3181 \\
3188 \\
\text { F3207 }\end{array}$ & $\begin{array}{l}\text { FC0472 / FC0458 } \\
\text { FC0469 / FC0468 } \\
\text { FC0450 / FC0473 }\end{array}$ & 1.998 & 1.998 & 1.997 \\
\hline L1311 & 2 GIS & $\begin{array}{l}\text { F3217 } \\
\text { F3218 }\end{array}$ & $\begin{array}{l}\text { FC0506 / FC0508 } \\
\text { FC0507 / FC0509 }\end{array}$ & 1.999 & 1.999 & 1.999 \\
\hline L1701 & 3 GIS & $\begin{array}{l}3090 \mathrm{~A} \\
3166 \\
3183\end{array}$ & $\begin{array}{l}\text { FC0482 / FC0487 } \\
\text { FC0485 / FC0488 } \\
\text { FC0492 / FC0493 }\end{array}$ & 2.000 & 1.999 & 1.999 \\
\hline L1702 & $\begin{array}{l}2 \\
\text { Modules }\end{array}$ & $\begin{array}{l}\text { F8138 } \\
\text { F8139 }\end{array}$ & $\begin{array}{l}\text { FC0471/FC0467/ } \\
\text { FC0497/FC0502 } \\
\text { FC0496/FC0499/ } \\
\text { FC0464/FC0465 }\end{array}$ & $1.999^{1}$ & $1.998^{1}$ & $1.998^{1}$ \\
\hline L1704 & $\begin{array}{l}2 \\
\text { Modules }\end{array}$ & $\begin{array}{l}\text { F8134 } \\
\text { F8135 }\end{array}$ & $\begin{array}{l}\text { FC0479/FC0480/ } \\
\text { FC0485/FC0488 } \\
\text { FC0482/FC0487/ } \\
\text { FC0461/FC0466 }\end{array}$ & $1.998^{1}$ & $1.997^{1}$ & $1.997^{1}$ \\
\hline L1707 & $\begin{array}{l}2 \\
\text { Modules }\end{array}$ & $\begin{array}{l}\text { F8132 } \\
\text { F8133 }\end{array}$ & $\begin{array}{l}\text { FC0501/FC0504/ } \\
\text { FC0469/FC0468 } \\
\text { FC0454/FC0446/ } \\
\text { FC0498/FC0505 }\end{array}$ & $1.999^{1}$ & $1.998^{1}$ & $1.999^{1}$ \\
\hline L1708 & $\begin{array}{l}2 \\
\text { Modules }\end{array}$ & $\begin{array}{l}\text { F8136 } \\
\text { F8137 }\end{array}$ & $\begin{array}{l}\text { FC0450/FC0473/ } \\
\text { FC0494/FC0503 } \\
\text { FC0492/FC0493/ } \\
\text { 30C0478/FC0477 }\end{array}$ & $1.998^{1}$ & $1.997^{1}$ & $1.996^{1}$ \\
\hline
\end{tabular}




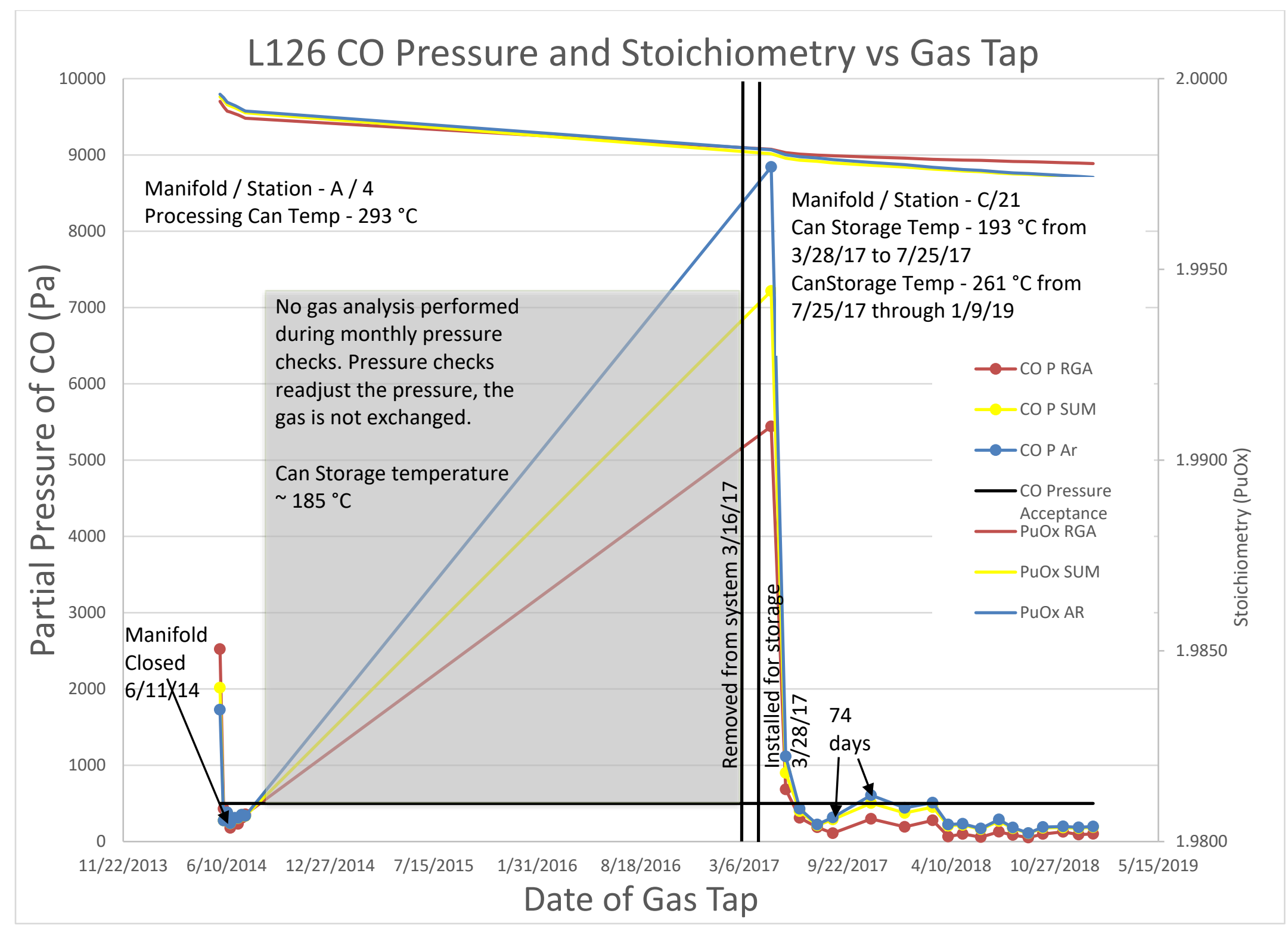

Figure 8 - MRM Can L126 CO Pressure and Stoichiometry vs Gas Tap 


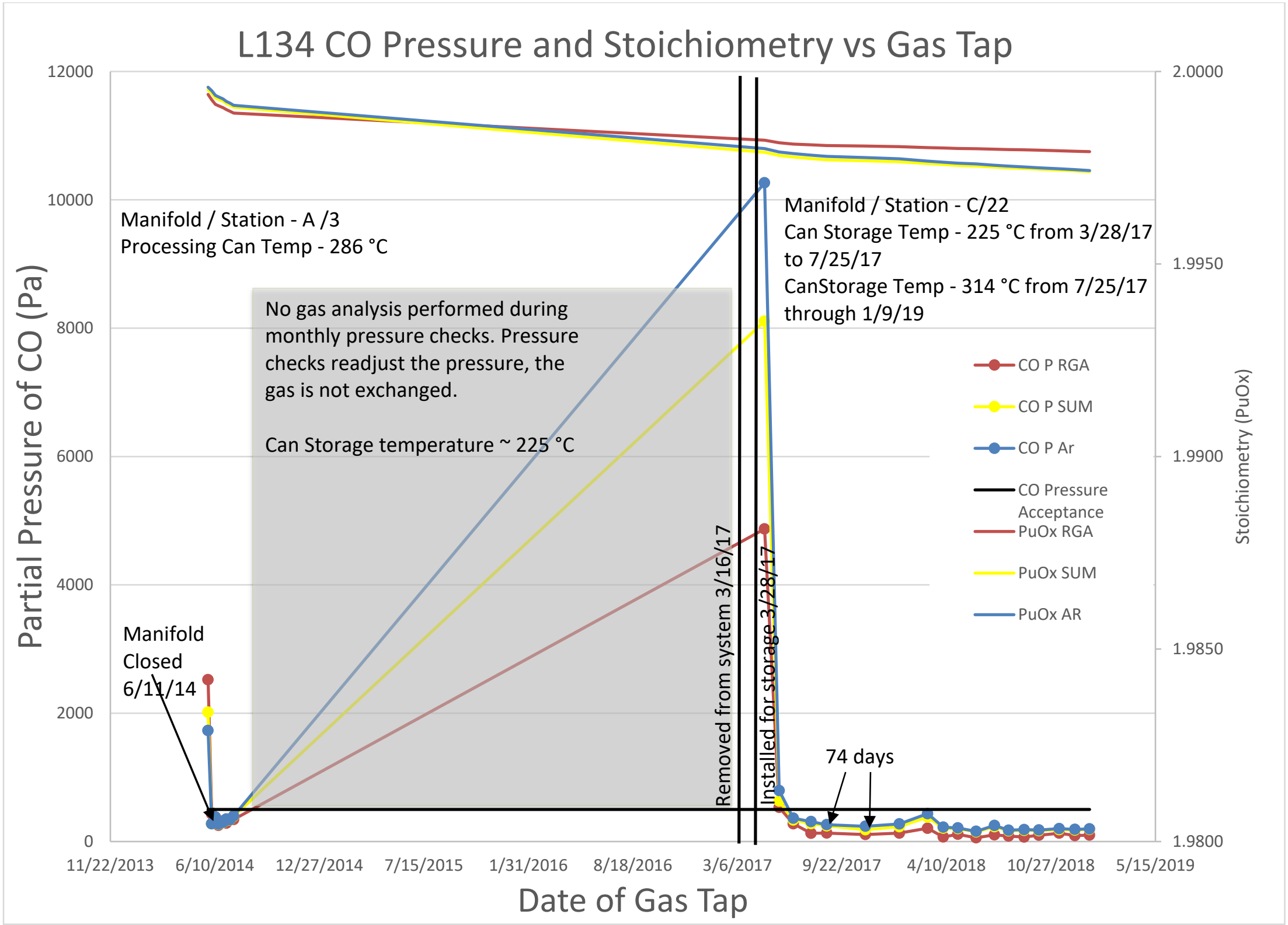

Figure 9 - MRM Can L134 CO Pressure and Stoichiometry vs Gas Tap 


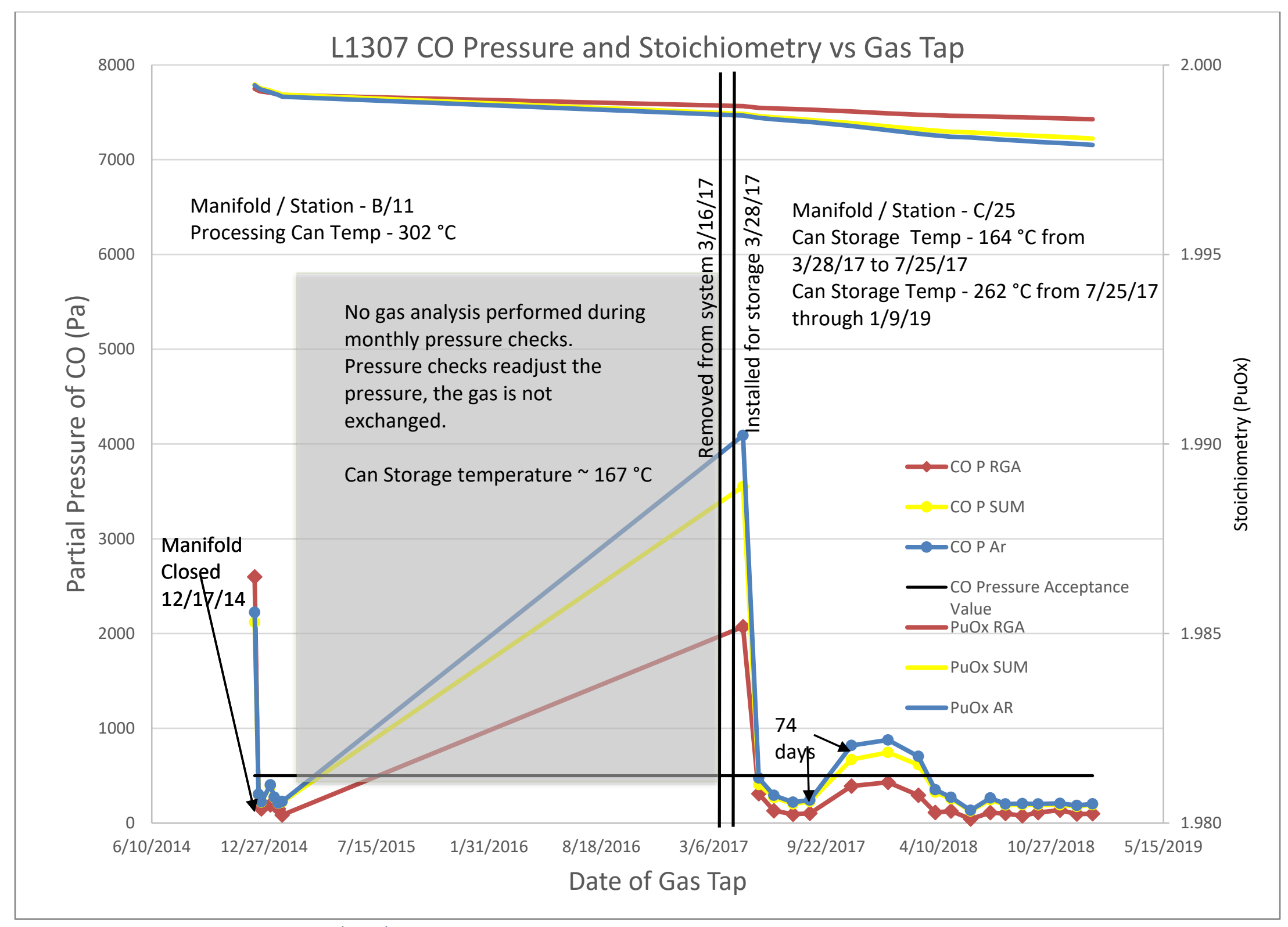

Figure 10 - MRM Can L1307 CO Pressure and Stoichiometry vs Gas Tap 


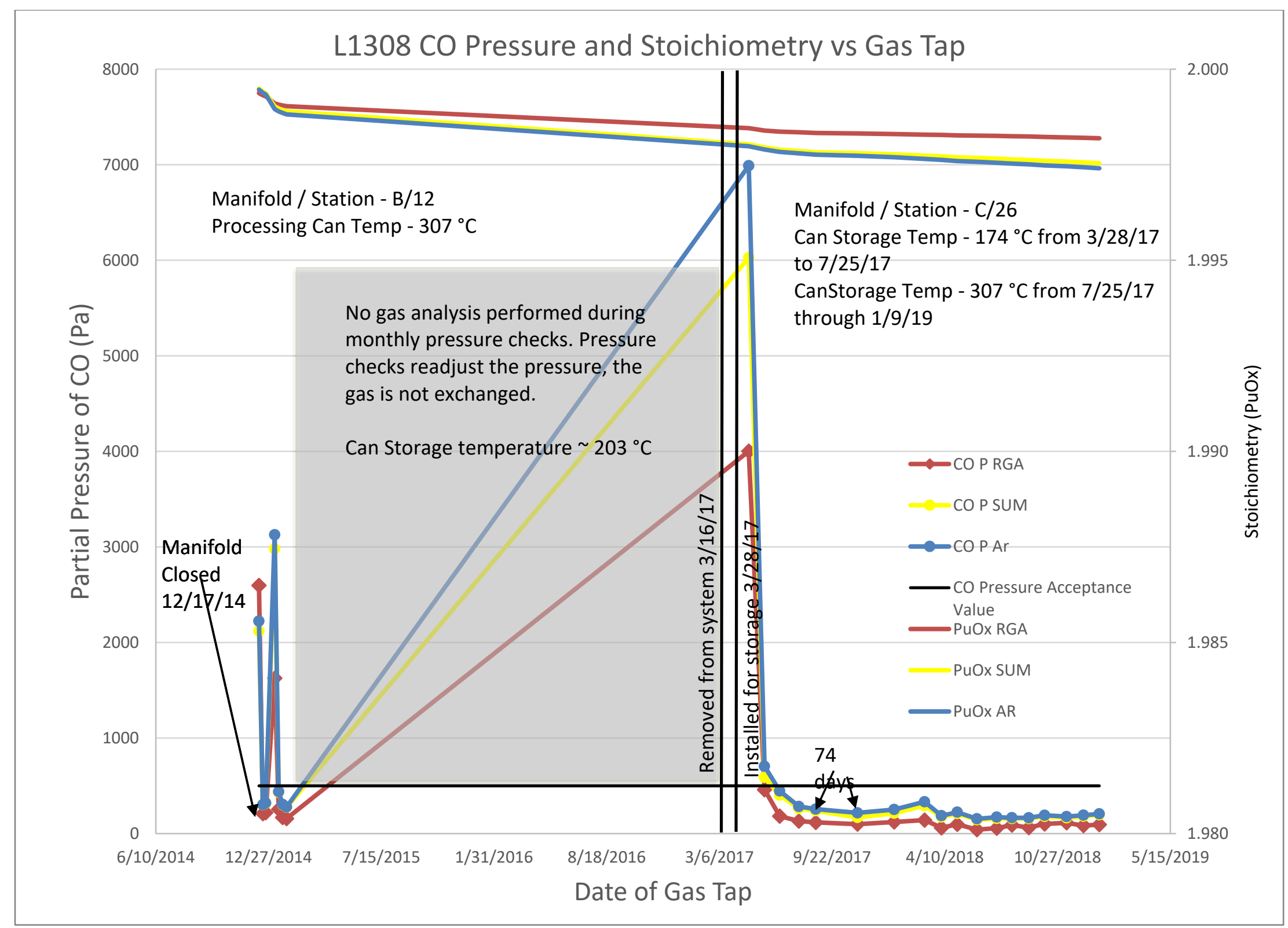

Figure 11 - MRM Can L1308 CO Pressure and Stoichiometry vs Gas Tap 


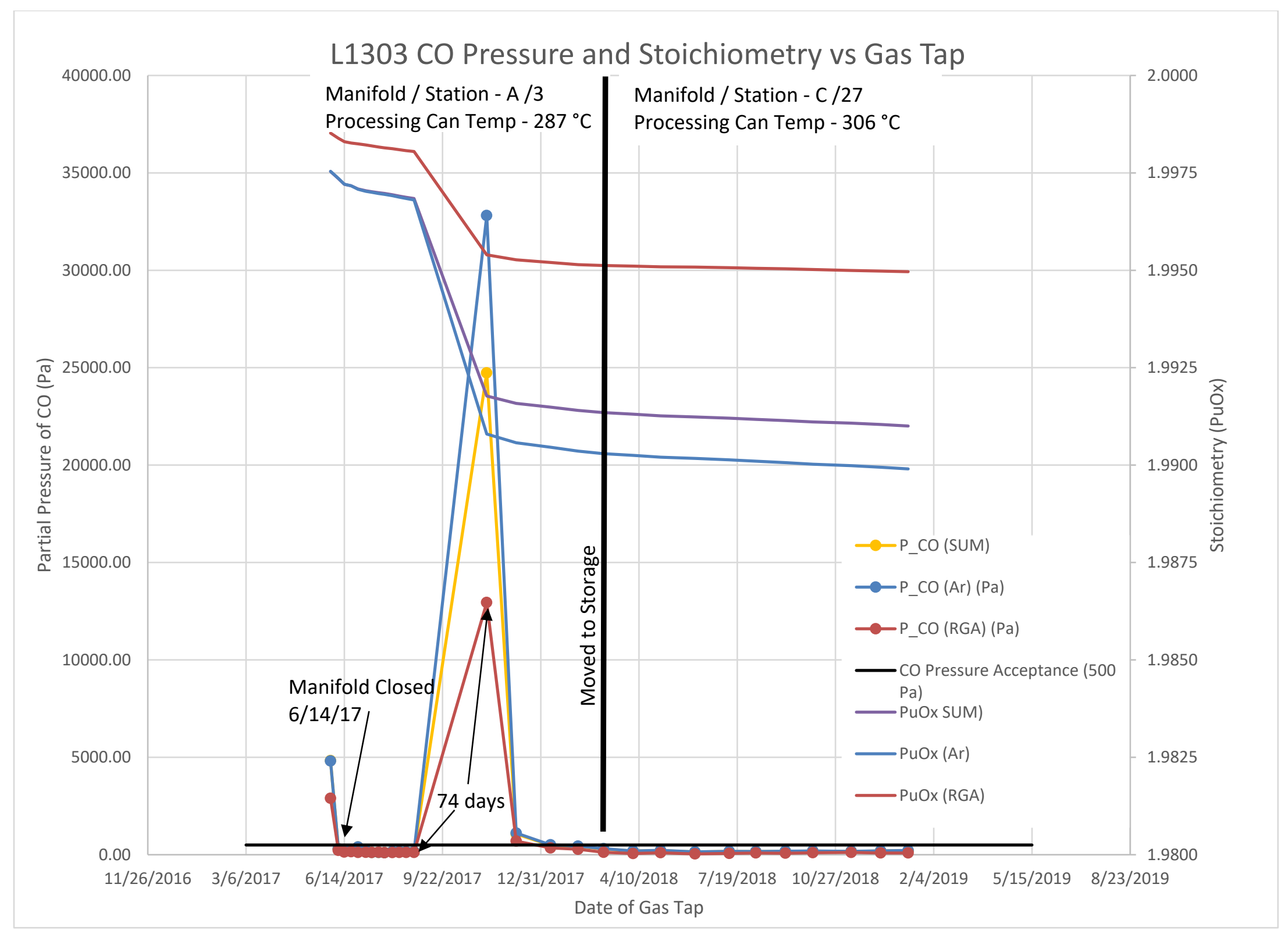

Figure 12 - MRM Can L1303 CO Pressure and Stoichiometry vs Gas Tap 


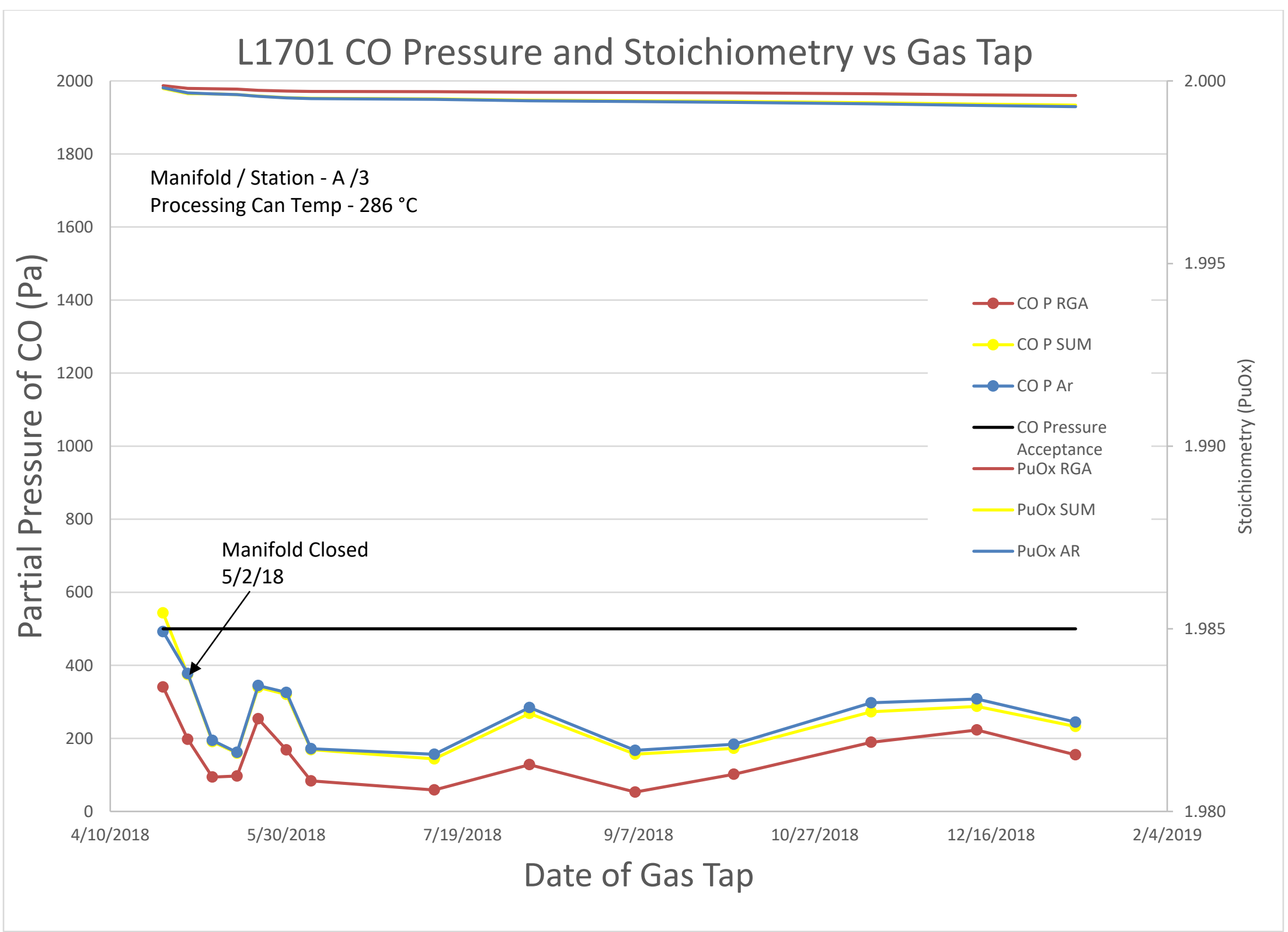

Figure 13 - MRM Can L1701 CO Pressure and Stoichiometry vs Gas Tap 


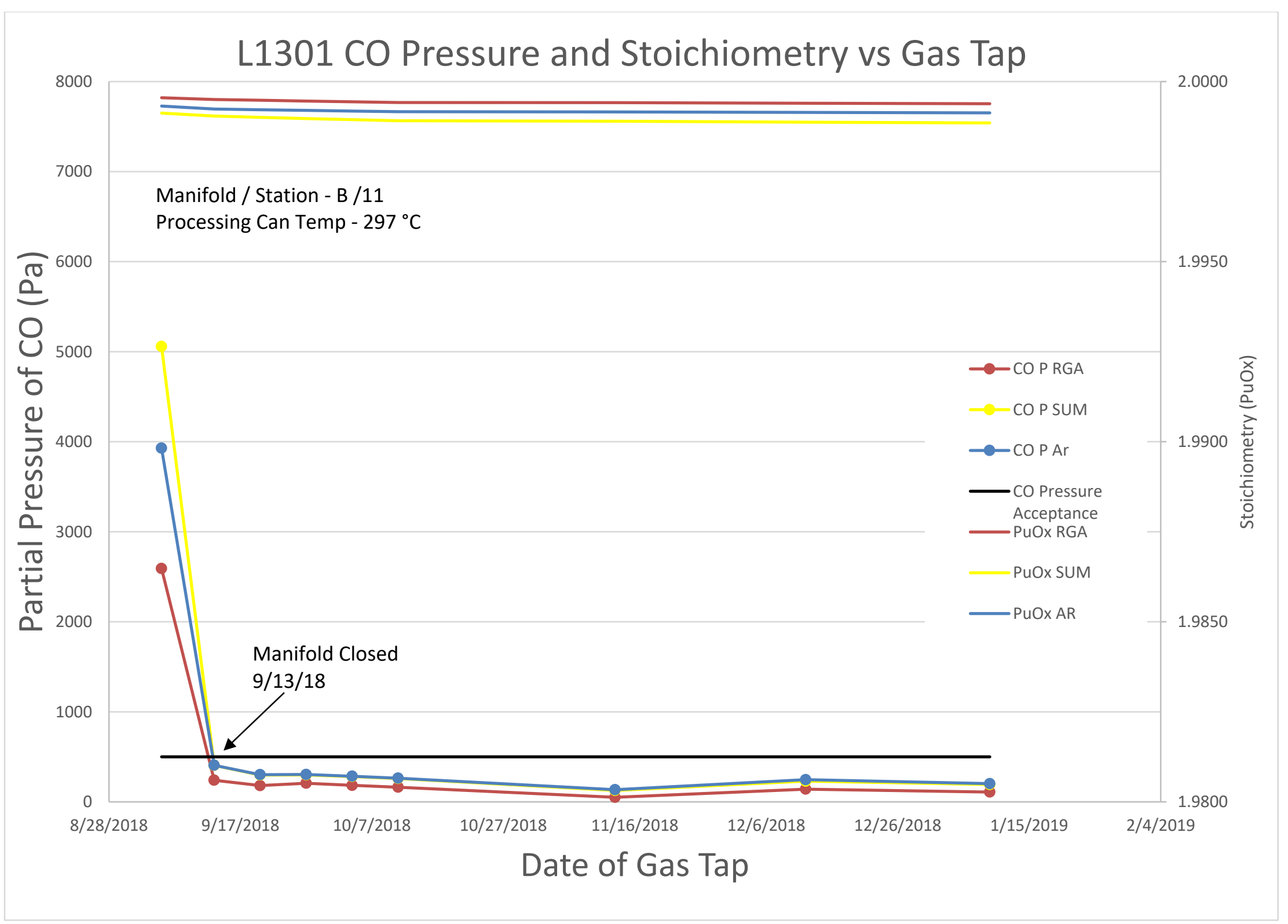

Figure 14 - MRM Can L1301 CO Pressure and Stoichiometry vs Gas Tap 


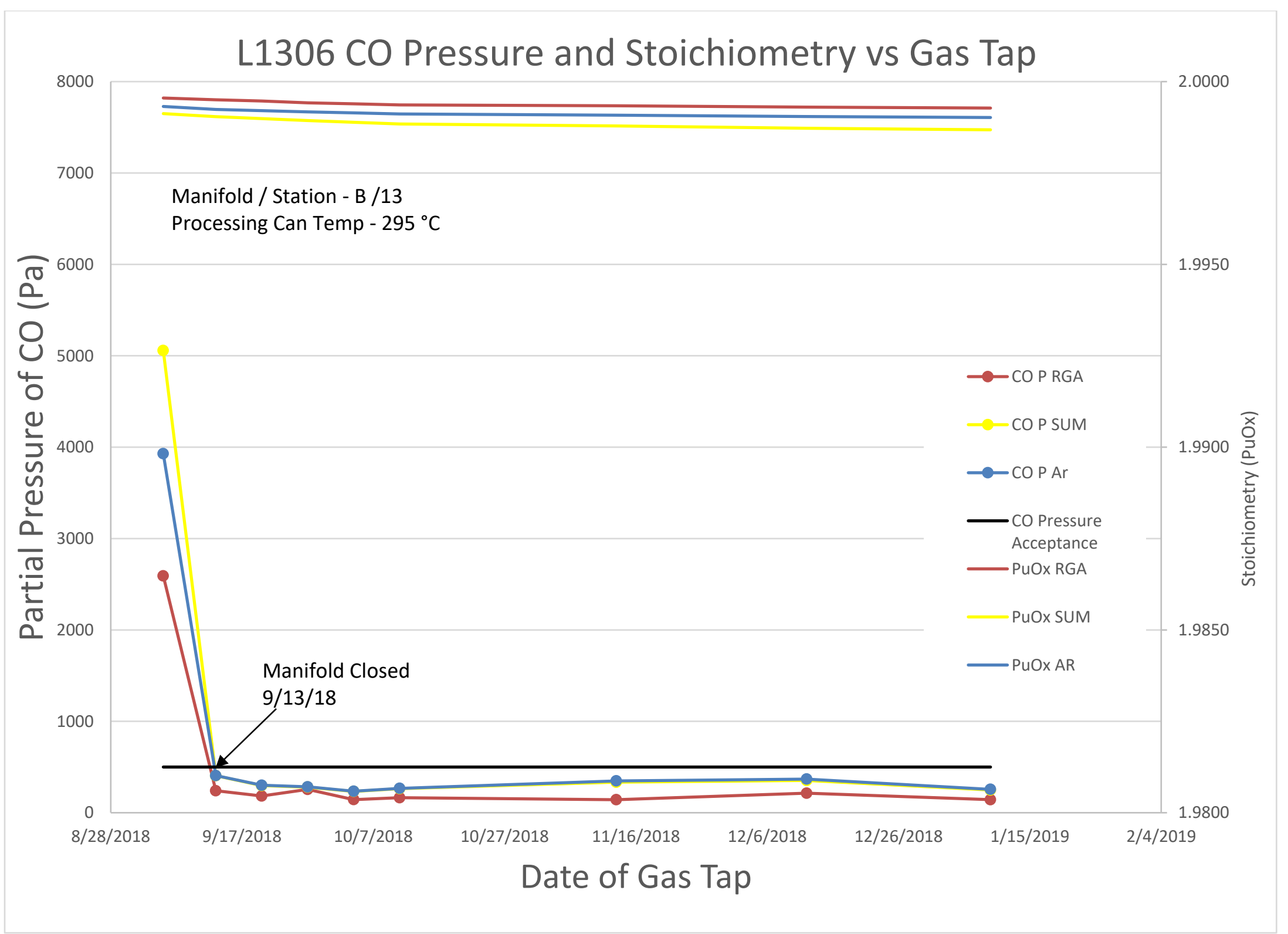

Figure 15 - MRM Can L1306 CO Pressure and Stoichiometry vs Gas Tap 


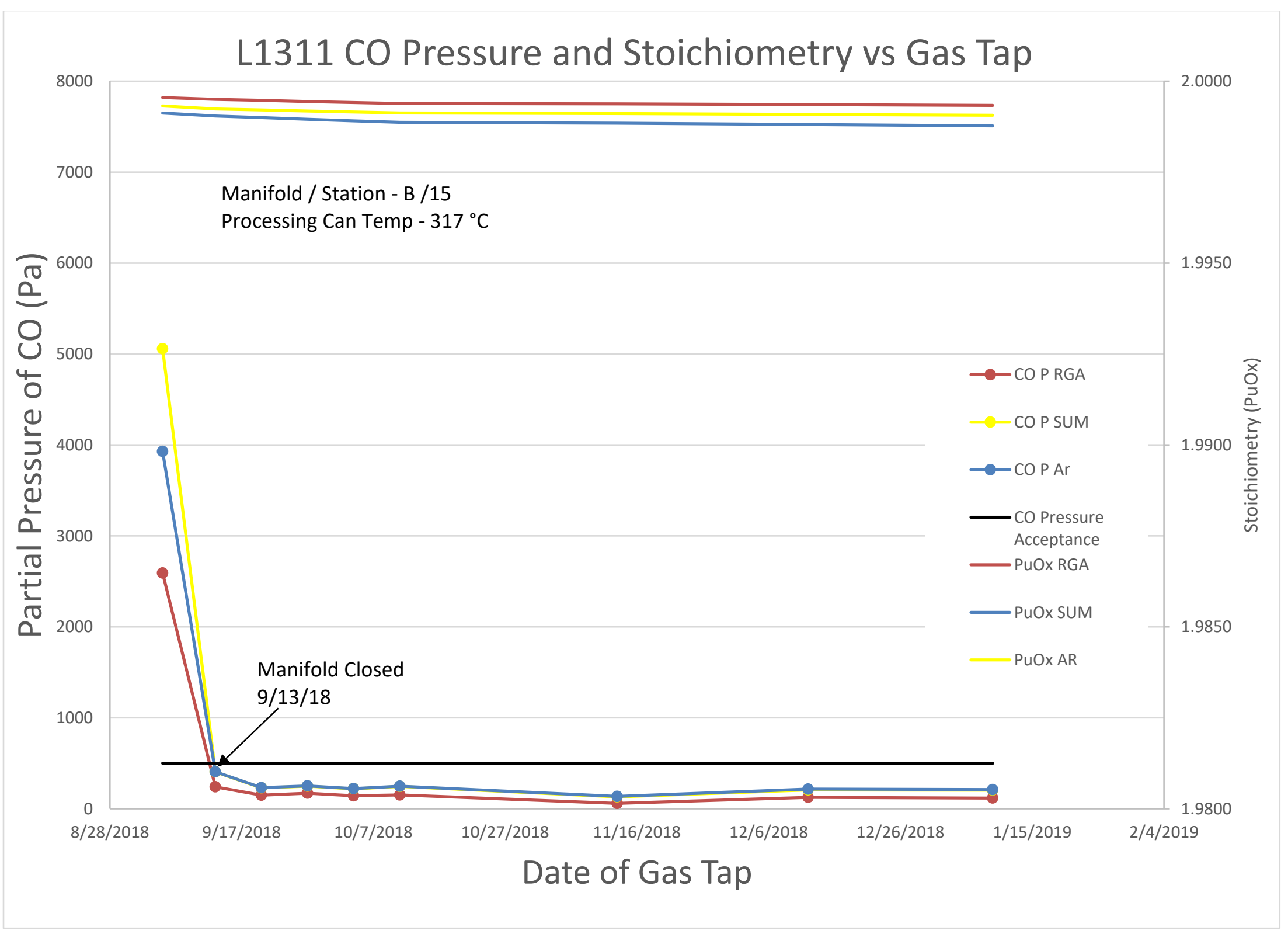

Figure 16 - MRM Can L1311 CO Pressure and Stoichiometry vs Gas Tap 


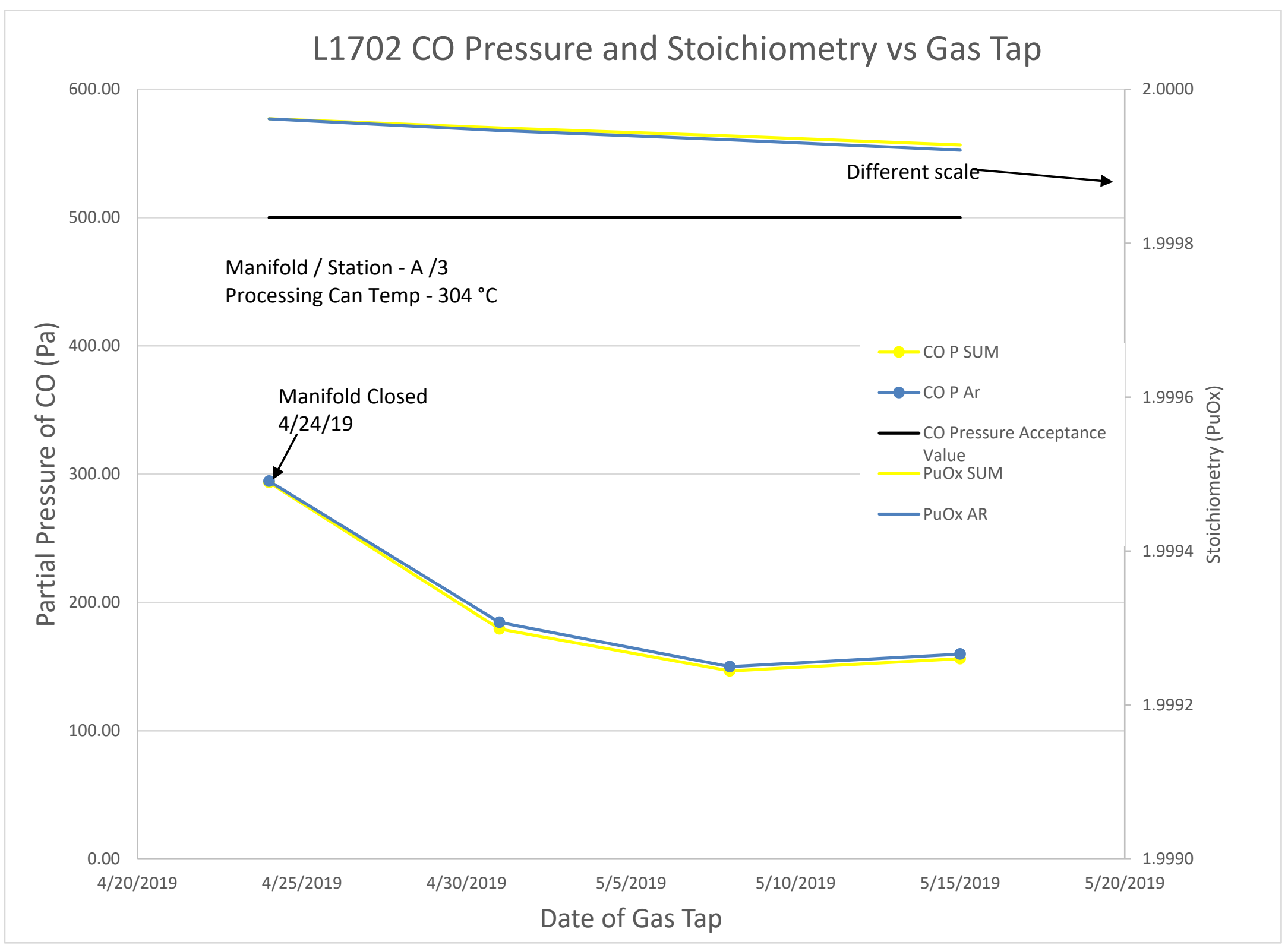

Figure 17 - MRM Can L1702 CO Pressure and Stoichiometry vs Gas Tap 


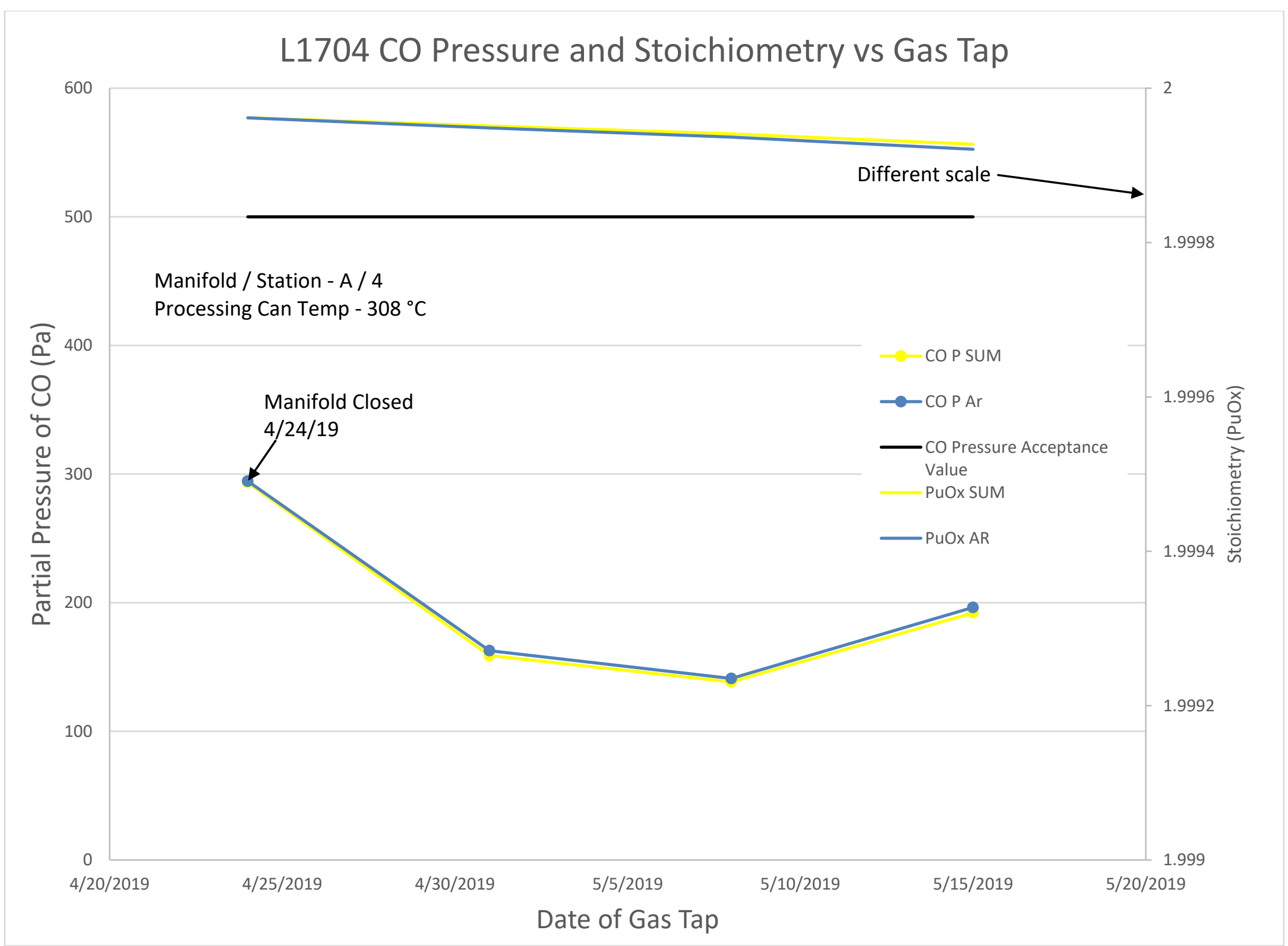

Figure 18 - MRM Can L1704 CO Pressure and Stoichiometry vs Gas Tap 


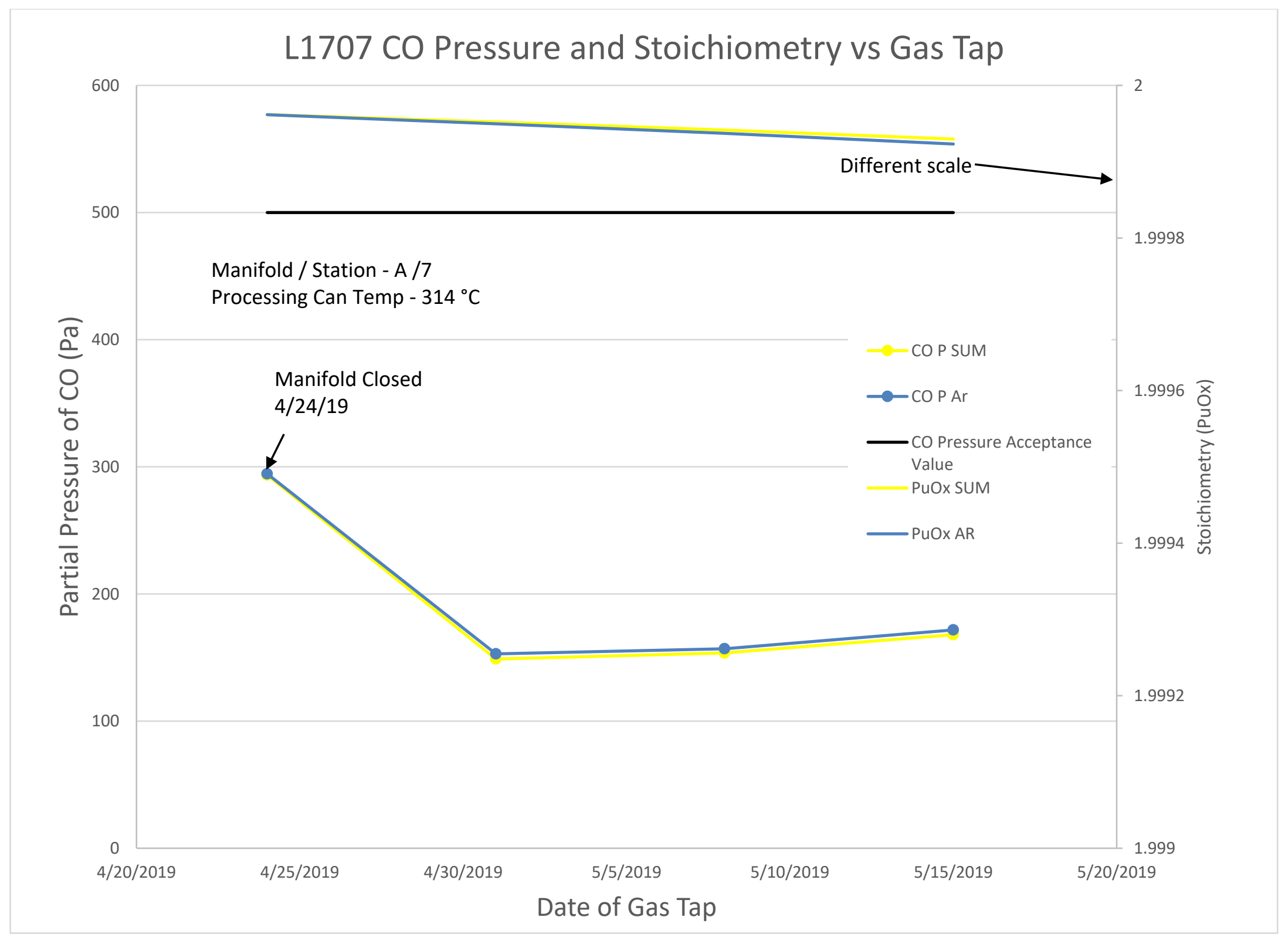

Figure 19 - MRM Can L1707 CO Pressure and Stoichiometry vs Gas Tap 


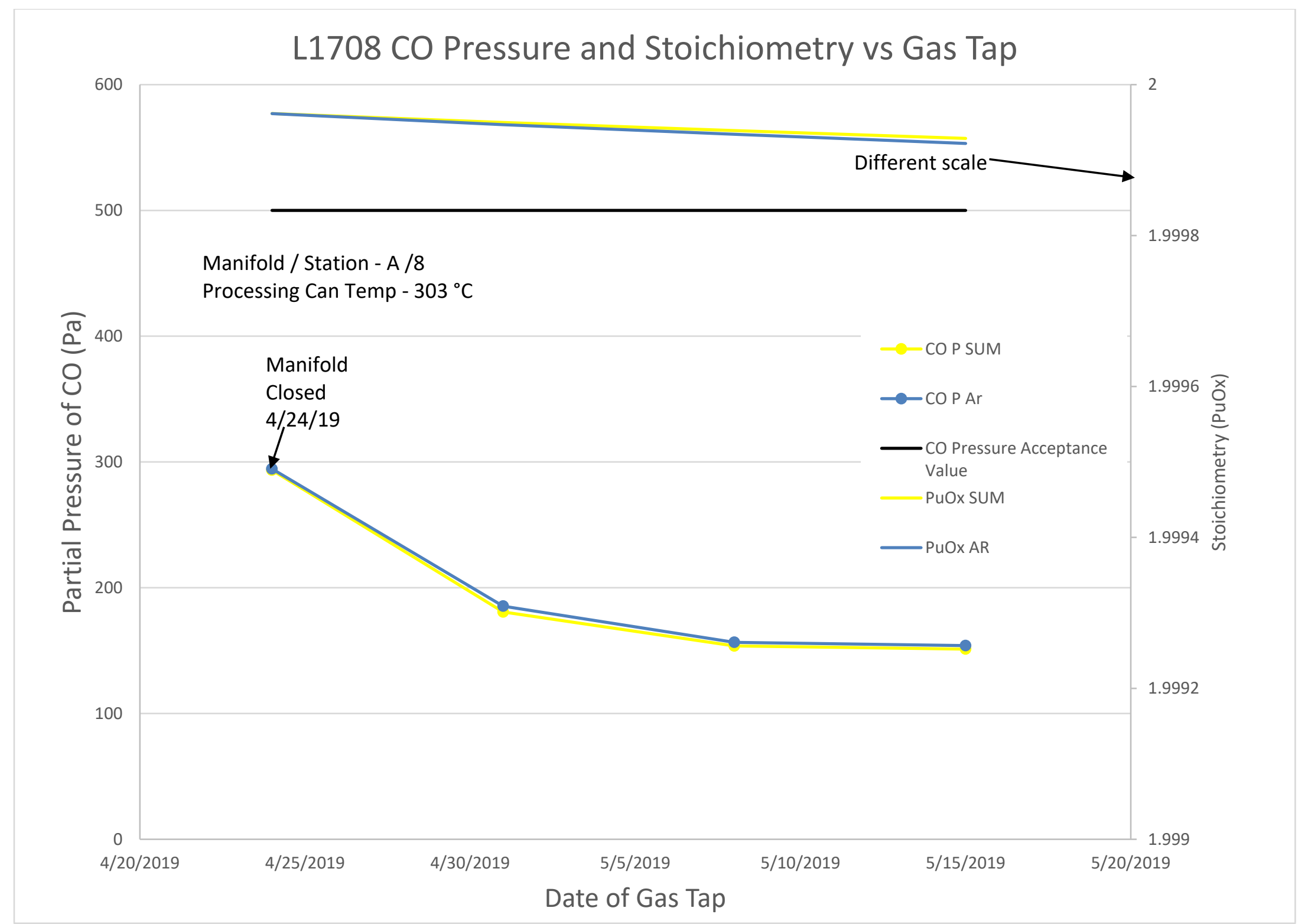

Figure 20 - MRM Can L1708 CO Pressure and Stoichiometry vs Gas Tap 


\section{Recommendations}

The following recommendations are derived from the preceding discussion:

Use the partial pressure of Ar instead of the overall RGA pressure value as the denominator in the acceptance criteria. The Ar value can be scaled against the charge pressure without needing to understand if other gases are evolving. The Ar is not reacting with other gases and there is no Ar source other than the charge pressure. In addition, the overall RGA pressure measurement is independent of the partial pressure measurements, not the summation of the partial pressures. Using the overall pressure and the partial pressure of $\mathrm{CO}$ adds another potential source of error.

Change the calibration method of the RGA to a more detailed procedure rather than the current, less consistent method. Set the Ar calibration factor to a nominal value and then ratio the other gases to match the molar concentrations reported on the gas analysis certificate. Tracking the ratio of the calibration factors between $\mathrm{CO}$ and $\mathrm{Ar}$ should be performed to determine if an acceptance criterion during calibration is necessary.

Evaluate if reduction of the fuel to a set value such as 1.98 should be the requirement. The benefits listed in the papers (Johnson 1982 and 1991) appear to have been met by the low level of reduction. Figure 21 published in the paper "Thermodynamic Modeling of the plutonium-Oxygen System" (Guéneau 2008) demonstrates multiple orders of magnitude lower oxygen potential with slightly substoichiometic oxygen values. A more detailed evaluation of the target value should be performed.

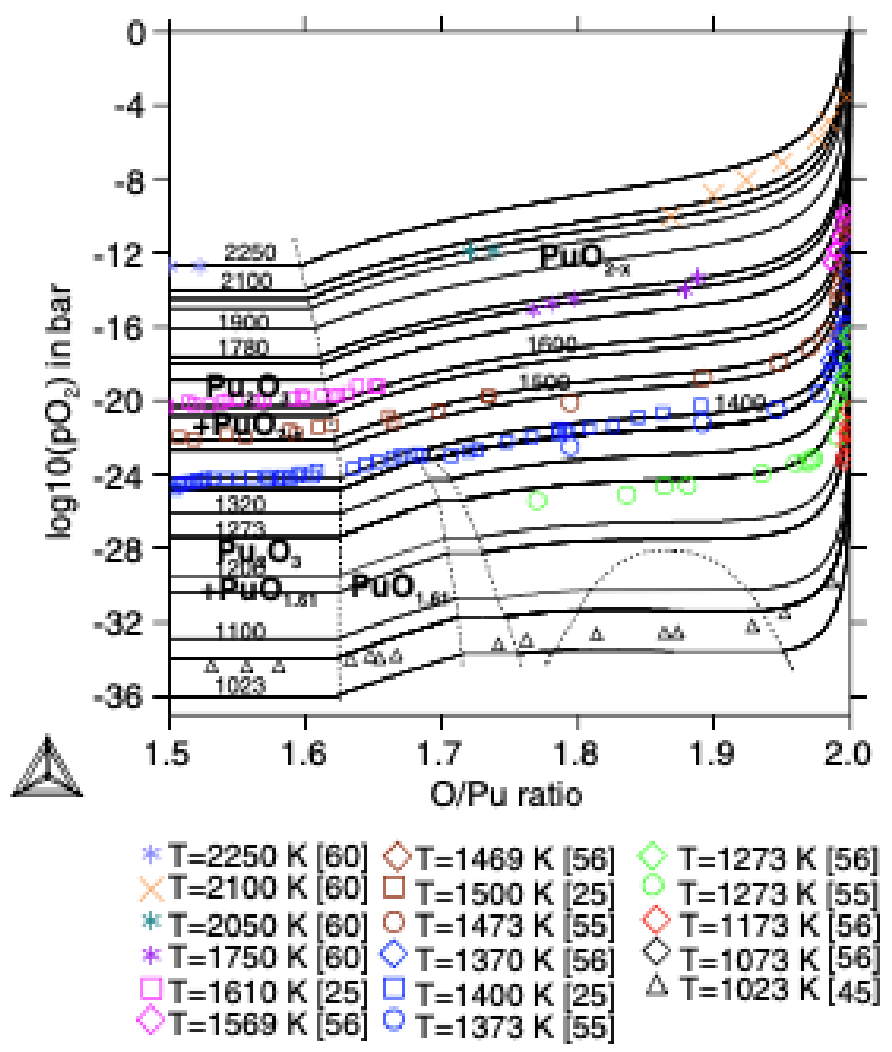

Figure 21 - Calculated $\mathrm{O}_{2}$ partial pressure versus O/Pu ratio (Fig 11 Guéneau 2008) 
If reduction of the fuel is a requirement, then a more robust method of felt packing that is more predictable and repeatable is warranted. Additionally, after processing, the level of reduction of the fuel should be calculated and documented to prevent process creep.

Current manual operations require $20 \pm 1$ psia is backfilled into the can, but in practice $20 \pm 0.1$ is used. The updated MRM system can achieve this pressure consistently and accurately. Consider eliminating the previous weeks fill pressure and use 20 psia for the basis of the acceptance calculation. At \pm 0.1 psi, the error in the calculated $\mathrm{CO}$ pressure is only $\pm 0.5 \%$.

Can temperature and felt packaging can have significant impact on fuel temperature and therefore, the thermodynamic limit of the reduction. More effort should be made evaluating the actual temperature of the fuel during the MRM process. This could be performed analytically using the ratio of $\mathrm{CO}$ to $\mathrm{CO}_{2}$, however, the current RGA used needs to be improved to lower the detectability range of $\mathrm{CO}_{2}$. Currently, the $\mathrm{CO}_{2}$ measurement appears to be below the detectability limit of the current RGA. Changing the MRM RGA to include an electron multiplier may allow for more accurate measurement of $\mathrm{CO}_{2}$ constituency.

Effort should be made to investigate the phenomenon where the $\mathrm{CO}$ partial pressure builds after periods of inactivity. CO build up during RTG pre-flight storage has been detected and could be detrimental to the internal components of past and future generator designs. Understanding this phenomenon may open processing methods that better control $\mathrm{CO}$ or be used as justification for periodic gas servicing, postfueling. 


\section{References}

Johnson, E. W. "Post-encapsulation plutonia reduction for Galileo and Ulysses GPHS modules: The module reduction and monitoring facility (MRMF)", Proceedings- Eight Symposium on Space Nuclear Power Systems, Pp. 1315, CONF-910116, Albuquerque, NM, 1991.

Johnson, E. W. "The Behavior of Oxygen Partial Pressure Over Slightly Substoichiometric Plutonia at 1410 K”, MLM-2980, Monsanto Research Corporation, Miamisburg, OH, Aug 1982.

Johnson, E. W. “Current Helium Venting Technology for ${ }^{238} \mathrm{PuO}_{2}$ Heat Sources,” Proceedings- Seventh Symposium on Space Nuclear Power Systems, Pp. 701, CONF-900109, Albuquerque, NM, 1990.

Merten, McDougal, Johnson, Worley, Diemunsch, Foose, Brehm and Kinard "Investigation of Apparent Fuel Clad Pressurization During the MRMF Process", September 28, 1995, EG\&G Mound Applied Technologies.

Johnson, E. "Spread of Plutonium in GPHS Modules: Evaluation of the QUAL RTG Graphites" July 21, 1992.

MD-21785, Issue 18, "Module Reduction and Monitoring Facility Operations", EG\&G Mound Applied Technologies.

PFR-MMRTG-FY19-1139 INL Problem Failure Report, "RGA Total Pressure Reported on RGA Printout is Dissimilar to the Calibrated Ion Gauge Indication"

INL-IP-FY13-913 INL Problem Failure Report "Pressure Was Underestimated During the Module Reduction Monitoring (MRM) Operation for F-8 \& MMRTG Fueled Modules"

LA-UR-00-4157 “Fabrication of Radioisotope Heat Sources for Space Missions", LANL

GESP 7253 "Carbon Monoxide Levels in Cassini RTGs”, April 1997, Lockheed Martin Missiles \& Space

Munns, K. “MRM Can Internal Volume Determination”, May 15, 2005

Guéneau, Chatillon and Sundman "Thermodynamic modelling of the plutonium-oxygen system” Journal of Nuclear Materials, vol. 378 (n 3). pp. 257-272. ISSN 0022-3115 2008 


\section{Appendix A}

Double release method of determining the initial gas pressure in an unknown volume at unknown average gas temperature 
Subscript " $C$ " is for the MRM can, subscript 1 is for the branch manifold and subscript 2 is for the main manifold. The manifold volumes are assumed to be evacuated at an ambient temperature of $298 \mathrm{~K}$, thus does not contribute any gas to the system.

$$
\begin{aligned}
& \mathrm{P}_{\mathrm{c}}=\mathrm{N} * \mathrm{R} * \mathrm{~T}_{\mathrm{c}} / \mathrm{V}_{\mathrm{c}}=\mathrm{N} * \mathrm{R} * \mathrm{X} \\
& \mathrm{N}=\text { number of moles of all gas species in the can } \\
& \mathrm{R}=8.314 \mathrm{~J} /\left(\mathrm{mol}^{*} \mathrm{~K}\right) \\
& \text { Let } \mathrm{X}=\mathrm{T}_{\mathrm{c}} / \mathrm{V}_{\mathrm{c}}
\end{aligned}
$$

Where

$$
\begin{aligned}
& \mathrm{T}_{\mathrm{c}}=\text { the average gas temperature of the can and flex line to the } \\
& \text { manifold solenoid }(\mathrm{K}) \\
& \mathrm{V}_{\mathrm{c}}=\text { the can and flex line volume }\left(\mathrm{m}^{3}\right)
\end{aligned}
$$

Open the can solenoid valve to combine $\mathrm{V}_{\mathrm{c}}$ and $\mathrm{V}_{1}$. The pressure $\left(\mathrm{P}_{1}\right)$ is read at the manifold. The same amount of gas, $\mathrm{N}$, is now in both volumes.

Where

$$
\mathrm{N}=\mathrm{P}_{1} * \mathrm{~V}_{1} /\left(\mathrm{R} * \mathrm{~T}_{\mathrm{amb}}\right)+\mathrm{P}_{1} /(\mathrm{R} * \mathrm{X})
$$

$$
\begin{aligned}
& \mathrm{V} 1 \text { = branch manifold volume } \\
& \text { Branch } \mathrm{A}=1.79 \mathrm{~L}=1.79 \times 10^{-3} \mathrm{~m}^{3} \\
& \text { Branch } \mathrm{B}=1.87 \mathrm{~L}=1.87 \times 10^{-3} \mathrm{~m}^{3} \\
& \text { Branch } \mathrm{C}=2.27 \mathrm{~L}=2.27 \times 10^{-3} \mathrm{~m}^{3}
\end{aligned}
$$

The branch manifold solenoid valve is open

$$
\mathrm{N}=\mathrm{P}_{2} *\left(\mathrm{~V}_{1}+\mathrm{V}_{2}\right) /\left(\mathrm{R} * \mathrm{~T}_{\mathrm{amb}}\right)+\mathrm{P}_{2} /(\mathrm{R} * \mathrm{X})
$$

The number of moles is equal in both cases

$$
\begin{aligned}
& \mathrm{P}_{2} *\left(\mathrm{~V}_{1}+\mathrm{V}_{2}\right) /\left(\mathrm{R} * \mathrm{~T}_{\mathrm{amb}}\right)+\mathrm{P}_{2} /(\mathrm{R} * \mathrm{X})=\mathrm{P}_{1} * \mathrm{~V}_{1} /\left(\mathrm{R} * \mathrm{~T}_{\mathrm{amb}}\right)+\mathrm{P}_{1} /(\mathrm{R} * \mathrm{X}) \\
& \mathrm{P}_{2} /(\mathrm{R} * \mathrm{X})-\mathrm{P}_{1} /(\mathrm{R} * \mathrm{X})=\mathrm{P}_{1} * \mathrm{~V}_{1} /\left(\mathrm{R}^{*} \mathrm{~T}_{\mathrm{amb}}\right)-\mathrm{P}_{2} *\left(\mathrm{~V}_{1}+\mathrm{V}_{2}\right) /\left(\mathrm{R}^{*} \mathrm{~T}_{\mathrm{amb}}\right) \\
& \left(\mathrm{P}_{2}-\mathrm{P}_{1}\right) /(\mathrm{R} * \mathrm{X})=\left(\mathrm{P}_{1} * \mathrm{~V}_{1}-\mathrm{P}_{2} *\left(\mathrm{~V}_{1}+\mathrm{V}_{2}\right)\right) /\left(\mathrm{R} * \mathrm{~T}_{\mathrm{amb}}\right) \\
& \mathrm{X}=\left(\mathrm{P}_{2}-\mathrm{P}_{1}\right) * \mathrm{~T}_{\mathrm{amb}} /\left(\mathrm{P}_{1} * \mathrm{~V}_{1}-\mathrm{P}_{2} *\left(\mathrm{~V}_{1}+\mathrm{V}_{2}\right)\right)
\end{aligned}
$$


Page intentionally left blank 


\section{Appendix B \\ MRM Can History}




\begin{tabular}{|c|c|c|c|c|c|}
\hline \multicolumn{6}{|c|}{ MRM Can Location History } \\
\hline MRM Can & Contents & GIS/MOD ID & FC ID & Location & Dates \\
\hline \multicolumn{6}{|c|}{ Retired Cans (Cans Opened for Assembly) } \\
\hline L126 & $3 \mathrm{GIS}$ & $\begin{array}{l}3173 \\
3174 \\
3180\end{array}$ & $\begin{array}{l}\text { FC0455 / FC0459 } \\
\text { FC0446 / FC0454 } \\
\text { FC0452 / FC0453 }\end{array}$ & $\begin{array}{l}\text { Manifold A Station } 4 \\
\text { Manifold C Station } 21\end{array}$ & $\begin{array}{l}\text { May 19, 2014 - March 16, } 2017 \\
\text { March 28, } 2017 \text { - March 7, } 2019\end{array}$ \\
\hline L134 & 3 GIS & $\begin{array}{l}3175 \\
3177 \\
3179\end{array}$ & $\begin{array}{l}\text { FC0448 / FC0442 } \\
\text { FC0441 / FC0457 } \\
\text { FC0449 / FC0451 }\end{array}$ & $\begin{array}{l}\text { Manifold A Station } 3 \\
\text { Manifold C Station } 22\end{array}$ & $\begin{array}{c}\text { May 19, } 2014-\text { March 16, } 2017 \\
\text { Mar 28, } 2017 \text { - Mar 12, } 2019\end{array}$ \\
\hline L1301 & 3 GIS & $\begin{array}{l}\text { F3219 } \\
\text { F3213 } \\
\text { F3214 }\end{array}$ & $\begin{array}{l}\text { FC0494 / FC0503 } \\
\text { FC0501 / FC0504 } \\
\text { FC0498 / FC0505 }\end{array}$ & Manifold B Station 11 & Aug 29, $2018-$ Feb 26, 2019 \\
\hline L1303 & 2 GIS & $\begin{array}{ll}\text { F3211 } \\
\text { F3212 }\end{array}$ & $\begin{array}{l}\text { FC0478 / FC0477 } \\
\text { FC0479 / FC0480 }\end{array}$ & $\begin{array}{l}\text { Manifold A Station } 3 \\
\text { Manifold C Station } 27\end{array}$ & $\begin{array}{l}\text { May 22, 2017 - March 6, } 2018 \\
\text { March 6, } 2018 \text { - Feb 26, } 2019\end{array}$ \\
\hline L1306 & 2 GIS & $\begin{array}{l}\text { F3215 } \\
\text { F3216 }\end{array}$ & $\begin{array}{l}\text { FC0496 / FC0499 } \\
\text { FC0497 / FC0502 }\end{array}$ & Manifold B Station 13 & Aug 29, $2018-$ Feb 26, 2019 \\
\hline L1307 & $3 \mathrm{GIS}$ & $\begin{array}{l}\text { F3208 } \\
\text { F3209 } \\
\text { F3210 }\end{array}$ & $\begin{array}{l}\text { FC0461 / FC0466 } \\
\text { FC0464 / FC0465 } \\
\text { FC0471 / FC0467 }\end{array}$ & $\begin{array}{l}\text { Manifold B Station } 11 \\
\text { Manifold C Station } 25\end{array}$ & $\begin{array}{l}\text { Dec 2, 2014 - March 16, } 2017 \\
\text { March 28, } 2017 \text { - Feb 26, } 2019\end{array}$ \\
\hline L1308 & $3 \mathrm{GIS}$ & $\begin{array}{l}3181 \\
3188 \\
F 3207\end{array}$ & $\begin{array}{l}\text { FC0472 / FC0458 } \\
\text { FC0469 / FC0468 } \\
\text { FC0450 / FC0473 }\end{array}$ & $\begin{array}{l}\text { Manifold B Station } 12 \\
\text { Manifold C Station } 26\end{array}$ & $\begin{array}{l}\text { Dec 2, 2014 - March 16, } 2017 \\
\text { March 28, } 2017 \text { - Feb 26, } 2019\end{array}$ \\
\hline L1311 & 2 GIS & $\begin{array}{l}\text { F3217 } \\
\text { F3218 }\end{array}$ & $\begin{array}{l}\text { FC0506 / FC0508 } \\
\text { FC0507 / FC0509 }\end{array}$ & Manifold B Station 15 & Aug 29, 2018 - Mar 12, 2019 \\
\hline L1701 & $3 \mathrm{GIS}$ & $\begin{array}{l}3090 A \\
3166 \\
3183\end{array}$ & $\begin{array}{l}\text { FC0482 / FC0487 } \\
\text { FC0485 / FC0488 } \\
\text { FC0492 / FC0493 }\end{array}$ & Manifold A Station 3 & April 18, 2018 - Feb 26, 2019 \\
\hline L1702 & 2 Module & $\begin{array}{l}\text { F8138 } \\
\text { F8139 }\end{array}$ & $\begin{array}{l}\text { FC0471/FC0467/FC0497/FC0502 } \\
\text { FC0496/FC0499/FC0464/FC0465 }\end{array}$ & Manifold A Station 3 & April 15, 2019 - Aug 6, 2019 \\
\hline L1704 & 2 Module & $\begin{array}{l}\text { F8134 } \\
\text { F8135 }\end{array}$ & $\begin{array}{l}\text { FC0479/FC0480/FC0485/FC0488 } \\
\text { FC0482/FC0487/FC0461/FC0466 }\end{array}$ & Manifold A Station 4 & April 15, 2019 - Aug 6, 2019 \\
\hline L1707 & 2 Module & $\begin{array}{l}\text { F8132 } \\
\text { F8133 }\end{array}$ & $\begin{array}{l}\text { FC0501/FC0504/FC0469/FC0468 } \\
\text { FC0454/FC0446/FC0498/FC0505 }\end{array}$ & Manifold A Station 7 & April 15, 2019 - Aug 6, 2019 \\
\hline L1708 & 2 Module & $\begin{array}{l}\text { F8136 } \\
\text { F8137 }\end{array}$ & $\begin{array}{l}\text { FC0450/FC0473/FC0494/FC0503 } \\
\text { FC0492/FC0493/FC0478/FC0477 }\end{array}$ & Manifold A Station 8 & April 15, 2019 - Aug 6, 2019 \\
\hline
\end{tabular}

Last updated: 11/14/2019 


\section{Appendix C \\ MRM RGA Data}




\begin{tabular}{|c|c|c|c|c|c|c|c|c|c|c|c|c|c|c|c|c|}
\hline Date & $\begin{array}{l}\text { Comment } \\
\mathrm{s}\end{array}$ & $\begin{array}{c}\text { MRM } \\
\text { Can } \\
\text { Sampled }\end{array}$ & $\begin{array}{l}\text { Total } \\
\text { press }\end{array}$ & Ch 1 & Ch 2 & $\mathrm{Ch} 3$ & Ch 4 & Ch 5 & Ch 6 & $\mathrm{Ch} 7$ & Ch 8 & Ch 9 & Ch 10 & Ch 11 & Ch 12 & $\begin{array}{c}\text { lon } \\
\text { pressure }\end{array}$ \\
\hline & & & $\begin{array}{c}\mathrm{AMU} / \mathrm{ch} \\
\mathrm{g}\end{array}$ & 4 & 28 & 40 & 44 & 20 & 16 & 17 & 18 & 32 & 36 & 12 & 14 & \\
\hline $6 / 17 / 2014$ & station 4 & L126 & $8.90 \mathrm{E}-06$ & $5.678 \mathrm{E}-08$ & $1.193 \mathrm{E}-08$ & $5.011 \mathrm{E}-06$ & $1.618 \mathrm{E}-09$ & 4.867E- -07 & & $9.045 \mathrm{E}-10$ & 5.617E- 09 & $3.332 \mathrm{E}-10$ & $3.713 \mathrm{E}-09$ & & & $5.80 \mathrm{E}-06$ \\
\hline $6 / 17 / 2014$ & station 4 & L126 & $7.80 \mathrm{E}-06$ & $5.198 \mathrm{E}-08$ & $1.013 \mathrm{E}-08$ & $5.843 \mathrm{E}-06$ & $1.618 \mathrm{E}-09$ & $5.401 \mathrm{E}-07$ & & $8.569 \mathrm{E}-10$ & $5.712 \mathrm{E}-09$ & $1.428 \mathrm{E}-10$ & $3.808 \mathrm{E}-09$ & & & $5.80 \mathrm{E}-06$ \\
\hline $6 / 26 / 2014$ & station 4 & L126 & $9.60 \mathrm{E}-06$ & $9.237 \mathrm{E}-08$ & $2.125 \mathrm{E}-08$ & $7.811 \mathrm{E}-06$ & 4.046E-09 & $6.119 \mathrm{E}-07$ & & $2.818 \mathrm{E}-09$ & $1.490 \mathrm{E}-08$ & $3.808 \mathrm{E}-10$ & $4.141 \mathrm{E}-09$ & & & $5.90 \mathrm{E}-06$ \\
\hline $6 / 26 / 2014$ & station 4 & L126 & $8.60 \mathrm{E}-06$ & $8.118 \mathrm{E}-08$ & $1.816 \mathrm{E}-08$ & $8.094 \mathrm{E}-06$ & 4.046E-09 & $6.340 \mathrm{E}-07$ & & $2.570 \mathrm{E}-09$ & 1.409E-08 & $3.332 \mathrm{E}-10$ & $4.046 \mathrm{E}-09$ & & & 5.90E-06 \\
\hline $7 / 2 / 2014$ & station 4 & L126 & $8.90 \mathrm{E}-06$ & $6.150 \mathrm{E}-08$ & $1.593 \mathrm{E}-08$ & $5.469 \mathrm{E}-06$ & $2.285 \mathrm{E}-09$ & $4.965 \mathrm{E}-07$ & & 2.237E-09 & $1.161 \mathrm{E}-08$ & $2.856 \mathrm{E}-10$ & 4.141E-09 & & & $6.00 \mathrm{E}-06$ \\
\hline $7 / 2 / 2014$ & station 4 & L126 & $7.90 \mathrm{E}-06$ & $5.503 \mathrm{E}-08$ & $1.319 \mathrm{E}-08$ & $5.900 \mathrm{E}-06$ & $1.713 \mathrm{E}-09$ & $5.336 \mathrm{E}-07$ & & $2.094 \mathrm{E}-09$ & $1.137 \mathrm{E}-08$ & $2.856 \mathrm{E}-10$ & $4.903 \mathrm{E}-09$ & & & $6.00 \mathrm{E}-06$ \\
\hline $7 / 9 / 2014$ & station 4 & L126 & $9.60 \mathrm{E}-06$ & $7.026 \mathrm{E}-08$ & $2.800 \mathrm{E}-08$ & $5.000 \mathrm{E}-07$ & $2.140 \mathrm{E}-09$ & $5.550 \mathrm{E}-07$ & & $3.142 \mathrm{E}-09$ & $1.578 \mathrm{E}-08$ & $4.204 \mathrm{E}-10$ & $5.900 \mathrm{E}-09$ & & & 5.90E-06 \\
\hline $7 / 9 / 2014$ & station 4 & L126 & $8.30 \mathrm{E}-06$ & $6.212 \mathrm{E}-08$ & $1.861 \mathrm{E}-08$ & $7.304 \mathrm{E}-06$ & 2.499E-09 & 5.690E-07 & & $2.856 \mathrm{E}-09$ & $1.485 \mathrm{E}-08$ & $3.332 \mathrm{E}-10$ & $6.284 \mathrm{E}-09$ & & & $5.90 \mathrm{E}-06$ \\
\hline $7 / 16 / 2014$ & station 4 & L126 & $1.00 \mathrm{E}-05$ & $7.883 \mathrm{E}-08$ & 2.217E-08 & $7.888 \mathrm{E}-06$ & $2.856 \mathrm{E}-09$ & $5.503 \mathrm{E}-07$ & & $3.618 \mathrm{E}-09$ & $1.885 \mathrm{E}-08$ & $5.236 \mathrm{E}-10$ & $6.617 \mathrm{E}-09$ & & & $6.10 \mathrm{E}-06$ \\
\hline $7 / 16 / 2014$ & station 4 & L126 & $8.40 \mathrm{E}-06$ & $7.026 \mathrm{E}-08$ & $2.168 \mathrm{E}-08$ & $8.883 \mathrm{E}-06$ & $2.856 \mathrm{E}-09$ & $5.645 \mathrm{E}-07$ & & $3.618 \mathrm{E}-09$ & $1.813 \mathrm{E}-08$ & $2.856 \mathrm{E}-10$ & 7.093E-09 & & & $6.10 \mathrm{E}-06$ \\
\hline $3 / 28 / 2017$ & Station 21 & L126 & $1.10 \mathrm{E}-05$ & $5.770 \mathrm{E}-06$ & 4.650E-08 & $4.350 \mathrm{E}-06$ & 1.477E-09 & $2.613 \mathrm{E}-07$ & & $4.284 \mathrm{E}-10$ & 2.427E-09 & $2.380 \mathrm{E}-10$ & 3.237E-09 & & & 4.50E-06 \\
\hline $4 / 27 / 2017$ & Station 21 & L126 & $1.00 \mathrm{E}-05$ & $9.908 \mathrm{E}-07$ & $3.926 \mathrm{E}-07$ & $6.155 \mathrm{E}-06$ & $3.213 \mathrm{E}-09$ & $4.008 \mathrm{E}-07$ & & $5.236 \mathrm{E}-10$ & $3.094 \mathrm{E}-09$ & $4.760 \mathrm{E}-10$ & $6.236 \mathrm{E}-09$ & $2.142 \mathrm{E}-09$ & 4.579E-09 & $5.90 \mathrm{E}-06$ \\
\hline $5 / 25 / 2017$ & Station 21 & L126 & 7.10E-06 & $1.012 \mathrm{E}-06$ & $3.507 \mathrm{E}-08$ & 4.352E-06 & $4.855 \mathrm{E}-10$ & 4.866E-07 & & $1.904 \mathrm{E}-10$ & $1.809 \mathrm{E}-09$ & 4.284E- 10 & $5.712 \mathrm{E}-09$ & $1.904 \mathrm{E}-10$ & $2.856 \mathrm{E}-10$ & $6.50 \mathrm{E}-06$ \\
\hline $6 / 21 / 2017$ & Station 21 & L126 & $1.10 \mathrm{E}-05$ & $5.934 \mathrm{E}-07$ & $2.483 \mathrm{E}-08$ & $7.962 \mathrm{E}-06$ & $1.047 \mathrm{E}-09$ & $6.046 \mathrm{E}-07$ & $9.521 \mathrm{E}-11$ & $9.521 \mathrm{E}-11$ & $2.189 \mathrm{E}-09$ & $1.904 \mathrm{E}-10$ & $4.951 \mathrm{E}-09$ & $9.521 \mathrm{E}-11$ & $2.380 \mathrm{E}-10$ & $8.00 \mathrm{E}-06$ \\
\hline $7 / 25 / 2017$ & Station 21 & L126 & $1.30 \mathrm{E}-05$ & $5.491 \mathrm{E}-07$ & $1.756 \mathrm{E}-08$ & $1.085 \mathrm{E}-05$ & $1.713 \mathrm{E}-09$ & $6.273 \mathrm{E}-07$ & $9.521 \mathrm{E}-11$ & $9.521 \mathrm{E}-11$ & $2.047 \mathrm{E}-09$ & $1.428 \mathrm{E}-10$ & $5.332 \mathrm{E}-09$ & $1.428 \mathrm{E}-10$ & $2.380 \mathrm{E}-10$ & $8.00 \mathrm{E}-06$ \\
\hline $8 / 24 / 2017$ & Station 21 & L126 & $1.50 \mathrm{E}-05$ & 4.670E- 07 & $1.188 \mathrm{E}-08$ & $5.185 \mathrm{E}-06$ & $1.385 \mathrm{E}-09$ & $5.550 \mathrm{E}-07$ & $1.428 \mathrm{E}-10$ & $1.428 \mathrm{E}-10$ & $1.856 \mathrm{E}-09$ & $1.428 \mathrm{E}-10$ & 4.903E-09 & 4.760E-11 & $1.428 \mathrm{E}-10$ & $8.30 \mathrm{E}-06$ \\
\hline $11 / 6 / 2017$ & Station 21 & L126 & $1.40 \mathrm{E}-05$ & $1.376 \mathrm{E}-06$ & $3.020 \mathrm{E}-08$ & $6.861 \mathrm{E}-06$ & $1.261 \mathrm{E}-09$ & $5.825 \mathrm{E}-07$ & 4.284E-10 & $8.569 \mathrm{E}-10$ & $6.188 \mathrm{E}-09$ & 4.284E-10 & $8.093 \mathrm{E}-09$ & $1.904 \mathrm{E}-10$ & $5.236 \mathrm{E}-10$ & $7.80 \mathrm{E}-06$ \\
\hline $1 / 10 / 2018$ & Station 21 & L126 & 1.60E-05 & 1.216E-06 & $2.224 \mathrm{E}-08$ & $6.962 \mathrm{E}-06$ & 1.475E-09 & $6.455 \mathrm{E}-07$ & $6.665 \mathrm{E}-10$ & $2.094 \mathrm{E}-09$ & 1.175E-08 & $5.236 \mathrm{E}-10$ & $7.855 \mathrm{E}-09$ & $2.380 \mathrm{E}-10$ & $6.188 \mathrm{E}-10$ & $8.30 \mathrm{E}-06$ \\
\hline $3 / 5 / 2018$ & Station 21 & L126 & $1.50 \mathrm{E}-05$ & $1.024 \mathrm{E}-06$ & $2.995 \mathrm{E}-08$ & $8.172 \mathrm{E}-06$ & $3.427 \mathrm{E}-09$ & $7.879 \mathrm{E}-07$ & $1.237 \mathrm{E}-09$ & $6.331 \mathrm{E}-09$ & $3.156 \mathrm{E}-08$ & $6.188 \mathrm{E}-10$ & $1.042 \mathrm{E}-08$ & $2.856 \mathrm{E}-10$ & $1.142 \mathrm{E}-09$ & $8.70 \mathrm{E}-06$ \\
\hline $4 / 4 / 2018$ & Station 21 & L126 & 2.30E-05 & $5.004 \mathrm{E}-07$ & $1.095 \mathrm{E}-08$ & $6.792 \mathrm{E}-06$ & $6.188 \mathrm{E}-10$ & $7.124 \mathrm{E}-07$ & $9.521 \mathrm{E}-11$ & $3.808 \mathrm{E}-10$ & $3.856 \mathrm{E}-09$ & $4.760 \mathrm{E}-10$ & $6.427 \mathrm{E}-09$ & 4.760E-11 & $2.856 \mathrm{E}-10$ & $1.40 \mathrm{E}-05$ \\
\hline $5 / 2 / 2018$ & Station 21 & L126 & $2.50 \mathrm{E}-05$ & $6.786 \mathrm{E}-07$ & $1.809 \mathrm{E}-08$ & $1.072 \mathrm{E}-05$ & 1.199E-09 & $9.372 \mathrm{E}-07$ & $2.380 \mathrm{E}-10$ & $1.142 \mathrm{E}-09$ & $7.188 \mathrm{E}-09$ & $6.665 \mathrm{E}-10$ & $8.378 \mathrm{E}-09$ & 4.760E-11 & 4.284E- 10 & $1.20 \mathrm{E}-05$ \\
\hline $6 / 6 / 2018$ & Station 21 & L126 & $3.00 \mathrm{E}-05$ & $6.187 \mathrm{E}-07$ & $1.262 \mathrm{E}-08$ & $1.021 \mathrm{E}-05$ & $6.855 \mathrm{E}-10$ & $7.972 \mathrm{E}-07$ & $1.428 \mathrm{E}-10$ & $3.332 \mathrm{E}-10$ & $3.380 \mathrm{E}-09$ & $3.808 \mathrm{E}-10$ & $1.009 \mathrm{E}-08$ & $1.428 \mathrm{E}-10$ & $1.904 \mathrm{E}-10$ & $1.30 \mathrm{E}-05$ \\
\hline $7 / 11 / 2018$ & Station 21 & L126 & $2.50 \mathrm{E}-05$ & $8.389 \mathrm{E}-07$ & $2.325 \mathrm{E}-08$ & $1.108 \mathrm{E}-05$ & 2.970E-09 & $8.225 \mathrm{E}-07$ & $5.712 \mathrm{E}-10$ & 4.332E-09 & 2.089E-08 & $5.712 \mathrm{E}-10$ & $1.133 \mathrm{E}-08$ & $1.904 \mathrm{E}-10$ & $5.712 \mathrm{E}-10$ & $1.30 \mathrm{E}-05$ \\
\hline $8 / 7 / 2018$ & Station 21 & L126 & $2.30 \mathrm{E}-05$ & $5.802 \mathrm{E}-07$ & $1.428 \mathrm{E}-08$ & $1.072 \mathrm{E}-05$ & $1.237 \mathrm{E}-09$ & $7.565 \mathrm{E}-07$ & $2.380 \mathrm{E}-10$ & 1.523E-09 & 8.759E-09 & $4.760 \mathrm{E}-10$ & $9.854 \mathrm{E}-09$ & $9.521 \mathrm{E}-11$ & $3.332 \mathrm{E}-10$ & $1.30 \mathrm{E}-05$ \\
\hline $9 / 6 / 2018$ & Station 21 & L126 & 2.00E-05 & 3.999E-07 & $7.598 \mathrm{E}-09$ & $9.457 \mathrm{E}-06$ & $9.521 \mathrm{E}-10$ & $9.098 \mathrm{E}-07$ & $1.428 \mathrm{E}-10$ & $9.521 \mathrm{E}-11$ & $2.047 \mathrm{E}-09$ & $1.904 \mathrm{E}-10$ & $9.759 \mathrm{E}-09$ & $9.521 \mathrm{E}-11$ & $1.428 \mathrm{E}-10$ & 1.40E-05 \\
\hline $10 / 4 / 2018$ & Station 21 & L126 & 2.30E-05 & $6.985 \mathrm{E}-07$ & $1.649 \mathrm{E}-08$ & $1.194 \mathrm{E}-05$ & $1.313 \mathrm{E}-09$ & $1.089 \mathrm{E}-06$ & $2.380 \mathrm{E}-10$ & $8.569 \mathrm{E}-10$ & $6.569 \mathrm{E}-09$ & $6.188 \mathrm{E}-10$ & $1.456 \mathrm{E}-08$ & $9.521 \mathrm{E}-11$ & $2.856 \mathrm{E}-10$ & 1.40E-05 \\
\hline $11 / 12 / 2018$ & Station 21 & L126 & $2.00 \mathrm{E}-05$ & $9.363 \mathrm{E}-07$ & $1.838 \mathrm{E}-08$ & $1.281 \mathrm{E}-05$ & $1.842 \mathrm{E}-09$ & $1.167 \mathrm{E}-06$ & $4.760 \mathrm{E}-10$ & $1.761 \mathrm{E}-09$ & $1.090 \mathrm{E}-08$ & $6.188 \mathrm{E}-10$ & $1.580 \mathrm{E}-08$ & $9.521 \mathrm{E}-11$ & $4.284 \mathrm{E}-10$ & $1.30 \mathrm{E}-05$ \\
\hline $12 / 12 / 2018$ & Station 21 & L126 & 2.40E-05 & $6.958 \mathrm{E}-07$ & $1.579 \mathrm{E}-08$ & $1.171 \mathrm{E}-05$ & $1.561 \mathrm{E}-09$ & $9.175 \mathrm{E}-07$ & 3.332E-10 & $1.333 \mathrm{E}-09$ & $8.474 \mathrm{E}-09$ & $7.141 \mathrm{E}-10$ & $1.475 \mathrm{E}-08$ & 4.760E-11 & $3.808 \mathrm{E}-10$ & 1.30E-05 \\
\hline
\end{tabular}




\begin{tabular}{|c|c|c|c|c|c|c|c|c|c|c|c|c|c|c|c|c|}
\hline Date & $\begin{array}{l}\text { Comment } \\
\mathrm{s}\end{array}$ & $\begin{array}{c}\text { MRM } \\
\text { Can } \\
\text { Sampled }\end{array}$ & $\begin{array}{l}\text { Total } \\
\text { press }\end{array}$ & Ch 1 & Ch 2 & Ch 3 & Ch 4 & Ch 5 & Ch 6 & $\mathrm{Ch} 7$ & Ch 8 & Ch 9 & Ch 10 & Ch 11 & Ch 12 & $\begin{array}{c}\text { Ion } \\
\text { pressure }\end{array}$ \\
\hline $1 / 9 / 2019$ & Station 21 & L126 & $1.90 \mathrm{E}-05$ & $3.976 \mathrm{E}-07$ & $1.385 \mathrm{E}-08$ & $9.723 \mathrm{E}-06$ & $1.028 \mathrm{E}-09$ & $9.446 \mathrm{E}-07$ & $1.904 \mathrm{E}-10$ & $5.712 \mathrm{E}-10$ & $4.903 \mathrm{E}-09$ & $7.141 \mathrm{E}-10$ & $1.294 \mathrm{E}-08$ & $9.521 \mathrm{E}-11$ & $2.380 \mathrm{E}-10$ & $1.20 \mathrm{E}-05$ \\
\hline $5 / 28 / 2014$ & Man A & $\begin{array}{l}\mathrm{L} 126 \\
\text { and } \\
\mathrm{L} 134\end{array}$ & $8.70 \mathrm{E}-06$ & $1.232 \mathrm{E}-06$ & $8.939 \mathrm{E}-08$ & $7.251 \mathrm{E}-06$ & $6.331 \mathrm{E}-09$ & $4.813 \mathrm{E}-07$ & $5.379 \mathrm{E}-09$ & $3.094 \mathrm{E}-09$ & $1.456 \mathrm{E}-08$ & $3.808 \mathrm{E}-10$ & $3.284 \mathrm{E}-09$ & $\begin{array}{c}0.000 E+0 \\
0\end{array}$ & $\begin{array}{c}0.000 \mathrm{E}+0 \\
0\end{array}$ & $5.70 \mathrm{E}-06$ \\
\hline $5 / 28 / 2014$ & Man A & $\begin{array}{l}\mathrm{L} 126 \\
\text { and } \\
\text { L134 }\end{array}$ & $8.10 \mathrm{E}-06$ & 1.199E-06 & $1.106 \mathrm{E}-07$ & $8.820 \mathrm{E}-06$ & $4.522 \mathrm{E}-09$ & $5.237 \mathrm{E}-07$ & $5.236 \mathrm{E}-09$ & $2.999 \mathrm{E}-09$ & $1.504 \mathrm{E}-08$ & $1.904 \mathrm{E}-10$ & $3.475 \mathrm{E}-09$ & $\begin{array}{c}0.000 E+0 \\
0\end{array}$ & $\begin{array}{c}0.000 E+0 \\
0\end{array}$ & $5.70 \mathrm{E}-06$ \\
\hline $6 / 4 / 2014$ & Man A & $\begin{array}{l}\mathrm{L} 126 \\
\text { and } \\
\text { L134 }\end{array}$ & $9.00 \mathrm{E}-06$ & $1.519 \mathrm{E}-07$ & $2.619 \mathrm{E}-08$ & $1.026 \mathrm{E}-05$ & $5.998 \mathrm{E}-09$ & $6.011 \mathrm{E}-07$ & $5.665 \mathrm{E}-09$ & $3.437 \mathrm{E}-09$ & $1.694 \mathrm{E}-08$ & $3.808 \mathrm{E}-10$ & $4.141 \mathrm{E}-09$ & $\begin{array}{c}0.000 \mathrm{E}+0 \\
0\end{array}$ & $\begin{array}{c}0.000 E+0 \\
0\end{array}$ & $6.00 \mathrm{E}-06$ \\
\hline $6 / 4 / 2014$ & Man A & $\begin{array}{l}\mathrm{L} 126 \\
\text { and } \\
\text { L134 }\end{array}$ & $7.80 \mathrm{E}-06$ & $1.527 \mathrm{E}-07$ & $2.399 \mathrm{E}-08$ & $1.199 \mathrm{E}-05$ & $5.998 \mathrm{E}-09$ & $6.619 \mathrm{E}-07$ & $5.570 \mathrm{E}-09$ & $3.284 \mathrm{E}-09$ & $1.699 \mathrm{E}-08$ & $2.856 \mathrm{E}-10$ & 4.141E-09 & $\begin{array}{c}0.000 E+0 \\
0\end{array}$ & $\begin{array}{c}0.000 E+0 \\
0\end{array}$ & $6.00 \mathrm{E}-06$ \\
\hline $6 / 11 / 2014$ & Man A & $\begin{array}{l}\mathrm{L} 126 \\
\text { and } \\
\text { L134 }\end{array}$ & $8.60 \mathrm{E}-06$ & $4.358 \mathrm{E}-08$ & $2.297 \mathrm{E}-08$ & $6.004 \mathrm{E}-06$ & $4.284 \mathrm{E}-09$ & $5.590 \mathrm{E}-07$ & $4.855 \mathrm{E}-09$ & $3.142 \mathrm{E}-09$ & $1.566 \mathrm{E}-08$ & $4.760 \mathrm{E}-10$ & 4.379E-09 & $\begin{array}{c}0.000 \mathrm{E}+0 \\
0\end{array}$ & $\begin{array}{c}0.000 E+0 \\
0\end{array}$ & $5.90 \mathrm{E}-06$ \\
\hline $6 / 11 / 2014$ & Man A & $\begin{array}{l}\mathrm{L} 126 \\
\text { and } \\
\mathrm{L} 134\end{array}$ & $7.30 \mathrm{E}-06$ & $4.318 \mathrm{E}-08$ & $1.971 \mathrm{E}-08$ & $7.108 \mathrm{E}-06$ & $4.284 \mathrm{E}-09$ & $6.054 \mathrm{E}-07$ & 4.617E-09 & 2.999E-09 & $1.580 \mathrm{E}-08$ & $2.854 \mathrm{E}-10$ & $4.284 \mathrm{E}-09$ & $\begin{array}{c}0.000 E+0 \\
0 \\
\end{array}$ & $\begin{array}{c}0.000 E+0 \\
0 \\
\end{array}$ & $5.90 \mathrm{E}-06$ \\
\hline $9 / 20 / 2018$ & Station 11 & L1301 & $2.20 \mathrm{E}-05$ & $1.629 \mathrm{E}-07$ & $2.881 \mathrm{E}-08$ & $1.314 \mathrm{E}-05$ & 4.070E-09 & 1.307E-06 & $7.141 \mathrm{E}-10$ & 4.427E-09 & $2.380 \mathrm{E}-08$ & $8.093 \mathrm{E}-10$ & $1.513 \mathrm{E}-08$ & $1.428 \mathrm{E}-10$ & $8.093 \mathrm{E}-10$ & $1.40 \mathrm{E}-05$ \\
\hline $9 / 27 / 2018$ & Station 11 & L1301 & $1.80 \mathrm{E}-05$ & $1.675 \mathrm{E}-07$ & 2.699E-08 & $1.224 \mathrm{E}-05$ & $2.999 \mathrm{E}-09$ & $1.207 \mathrm{E}-06$ & $5.712 \mathrm{E}-10$ & $3.760 \mathrm{E}-09$ & $2.013 \mathrm{E}-08$ & $8.569 \mathrm{E}-10$ & $1.409 \mathrm{E}-08$ & $1.428 \mathrm{E}-10$ & $6.188 \mathrm{E}-10$ & $1.30 \mathrm{E}-05$ \\
\hline $10 / 4 / 2018$ & Station 11 & L1301 & $2.10 \mathrm{E}-05$ & 1.697E-07 & $2.799 \mathrm{E}-08$ & $1.356 \mathrm{E}-05$ & $3.722 \mathrm{E}-09$ & $1.316 \mathrm{E}-06$ & $6.188 \mathrm{E}-10$ & 4.237E-09 & $2.270 \mathrm{E}-08$ & $7.141 \mathrm{E}-10$ & $1.513 \mathrm{E}-08$ & $1.904 \mathrm{E}-10$ & $5.712 \mathrm{E}-10$ & $1.50 \mathrm{E}-05$ \\
\hline $10 / 11 / 2018$ & Station 11 & L1301 & $2.50 \mathrm{E}-05$ & $2.106 \mathrm{E}-07$ & $2.949 \mathrm{E}-08$ & $1.545 \mathrm{E}-05$ & 4.475E-09 & $1.398 \mathrm{E}-06$ & $6.665 \mathrm{E}-10$ & $4.046 \mathrm{E}-09$ & $2.237 \mathrm{E}-08$ & $7.141 \mathrm{E}-10$ & $1.709 \mathrm{E}-08$ & $1.428 \mathrm{E}-10$ & $5.236 \mathrm{E}-10$ & $1.50 \mathrm{E}-05$ \\
\hline $11 / 13 / 2018$ & Station 11 & L1301 & $2.20 \mathrm{E}-05$ & $5.414 \mathrm{E}-07$ & $8.169 \mathrm{E}-09$ & $8.233 \mathrm{E}-06$ & $2.047 \mathrm{E}-10$ & $7.076 \mathrm{E}-07$ & $9.521 \mathrm{E}-11$ & $2.380 \mathrm{E}-10$ & $2.713 \mathrm{E}-09$ & $3.808 \mathrm{E}-10$ & $9.426 \mathrm{E}-09$ & $9.521 \mathrm{E}-11$ & $2.856 \mathrm{E}-10$ & 1.30E-05 \\
\hline $12 / 12 / 2018$ & Station 11 & L1301 & $2.20 \mathrm{E}-05$ & 8.607E-07 & $2.243 \mathrm{E}-08$ & $1.261 \mathrm{E}-05$ & $3.318 \mathrm{E}-09$ & $9.856 \mathrm{E}-07$ & $6.188 \mathrm{E}-10$ & $3.380 \mathrm{E}-09$ & $1.780 \mathrm{E}-08$ & $7.141 \mathrm{E}-10$ & $1.556 \mathrm{E}-08$ & $9.521 \mathrm{E}-11$ & $5.236 \mathrm{E}-10$ & $1.30 \mathrm{E}-05$ \\
\hline $1 / 9 / 2019$ & Station 11 & L1301 & $2.00 \mathrm{E}-05$ & $4.829 \mathrm{E}-07$ & $1.571 \mathrm{E}-08$ & $1.078 \mathrm{E}-05$ & $1.371 \mathrm{E}-09$ & $1.035 \mathrm{E}-06$ & $2.856 \mathrm{E}-10$ & $8.569 \mathrm{E}-10$ & $6.474 \mathrm{E}-09$ & $8.569 \mathrm{E}-10$ & $1.413 \mathrm{E}-08$ & $9.521 \mathrm{E}-11$ & $3.808 \mathrm{E}-10$ & $1.20 \mathrm{E}-05$ \\
\hline 9/5/2018 & Man B & $\begin{array}{l}\text { L1301, } \\
\text { L1306, } \\
\text { L1311 }\end{array}$ & $2.00 \mathrm{E}-05$ & $6.924 \mathrm{E}-07$ & $2.647 \mathrm{E}-07$ & $9.290 \mathrm{E}-06$ & $1.428 \mathrm{E}-09$ & $9.748 \mathrm{E}-07$ & 3.332E-10 & $1.428 \mathrm{E}-10$ & $2.475 \mathrm{E}-09$ & $5.236 \mathrm{E}-10$ & $1.014 \mathrm{E}-08$ & $1.047 \mathrm{E}-09$ & $6.665 \mathrm{E}-10$ & $1.40 \mathrm{E}-05$ \\
\hline $9 / 13 / 2018$ & Man B & $\begin{array}{l}\text { L1301, } \\
\text { L1306, } \\
\text { L1311 }\end{array}$ & $2.10 \mathrm{E}-05$ & $6.898 \mathrm{E}-08$ & $3.645 \mathrm{E}-08$ & $1.236 \mathrm{E}-05$ & $3.480 \mathrm{E}-09$ & $1.208 \mathrm{E}-06$ & $5.712 \mathrm{E}-10$ & $3.475 \mathrm{E}-09$ & $1.932 \mathrm{E}-08$ & $9.045 \mathrm{E}-10$ & $1.561 \mathrm{E}-08$ & $2.380 \mathrm{E}-10$ & $6.188 \mathrm{E}-10$ & $1.40 \mathrm{E}-05$ \\
\hline $5 / 31 / 2017$ & Station 3 & L1303 & $8.70 \mathrm{E}-06$ & $1.013 \mathrm{E}-07$ & $1.727 \mathrm{E}-07$ & $4.945 \mathrm{E}-06$ & $8.426 \mathrm{E}-10$ & $5.309 \mathrm{E}-07$ & $6.665 \mathrm{E}-10$ & $2.856 \mathrm{E}-10$ & $1.904 \mathrm{E}-09$ & $4.284 \mathrm{E}-10$ & $5.189 \mathrm{E}-09$ & $1.094 \mathrm{E}-09$ & $6.665 \mathrm{E}-10$ & $6.90 \mathrm{E}-06$ \\
\hline $5 / 31 / 2017$ & Station 3 & L1303 & $8.90 \mathrm{E}-06$ & $9.730 \mathrm{E}-08$ & $2.179 \mathrm{E}-07$ & $5.295 \mathrm{E}-06$ & 5.617E-10 & $5.484 \mathrm{E}-07$ & $7.141 \mathrm{E}-10$ & $9.521 \mathrm{E}-11$ & $2.047 \mathrm{E}-09$ & $4.760 \mathrm{E}-10$ & $5.332 \mathrm{E}-09$ & $1.237 \mathrm{E}-09$ & $8.093 \mathrm{E}-10$ & $6.90 \mathrm{E}-06$ \\
\hline $6 / 8 / 2017$ & Station 3 & L1303 & $8.50 \mathrm{E}-06$ & $1.666 \mathrm{E}-08$ & $1.366 \mathrm{E}-08$ & $6.168 \mathrm{E}-06$ & $1.285 \mathrm{E}-09$ & $5.921 \mathrm{E}-07$ & $1.428 \mathrm{E}-10$ & $1.904 \mathrm{E}-10$ & $2.523 \mathrm{E}-09$ & $2.380 \mathrm{E}-10$ & $5.474 \mathrm{E}-09$ & $1.428 \mathrm{E}-10$ & $2.856 \mathrm{E}-10$ & 7.40E-06 \\
\hline $6 / 8 / 2017$ & Station 3 & L1303 & $8.40 \mathrm{E}-06$ & $1.866 \mathrm{E}-08$ & $1.759 \mathrm{E}-08$ & 6.687E-06 & $8.569 \mathrm{E}-10$ & $6.315 \mathrm{E}-07$ & $2.856 \mathrm{E}-10$ & $1.904 \mathrm{E}-10$ & $2.427 \mathrm{E}-09$ & $3.332 \mathrm{E}-10$ & $6.046 \mathrm{E}-09$ & $9.521 \mathrm{E}-11$ & $2.380 \mathrm{E}-10$ & $7.30 \mathrm{E}-06$ \\
\hline 6/8/2017 & Station 3 & L1303 & $8.30 \mathrm{E}-06$ & $1.799 \mathrm{E}-08$ & $2.309 \mathrm{E}-08$ & $7.448 \mathrm{E}-06$ & $8.569 \mathrm{E}-10$ & $6.422 \mathrm{E}-07$ & $2.380 \mathrm{E}-10$ & $9.521 \mathrm{E}-11$ & $2.570 \mathrm{E}-09$ & $5.712 \mathrm{E}-10$ & $6.379 \mathrm{E}-09$ & $9.521 \mathrm{E}-11$ & $3.808 \mathrm{E}-10$ & $7.30 \mathrm{E}-06$ \\
\hline $6 / 14 / 2017$ & Station 3 & L1303 & $1.00 \mathrm{E}-05$ & $1.399 \mathrm{E}-08$ & $9.854 \mathrm{E}-09$ & $5.940 \mathrm{E}-06$ & $1.333 \mathrm{E}-09$ & $6.492 \mathrm{E}-06$ & $1.428 \mathrm{E}-10$ & $1.904 \mathrm{E}-10$ & $2.808 \mathrm{E}-09$ & $1.904 \mathrm{E}-10$ & $3.570 \mathrm{E}-09$ & $4.760 \mathrm{E}-11$ & $2.856 \mathrm{E}-10$ & $8.00 \mathrm{E}-06$ \\
\hline
\end{tabular}




\begin{tabular}{|c|c|c|c|c|c|c|c|c|c|c|c|c|c|c|c|c|}
\hline Date & $\begin{array}{l}\text { Comment } \\
\mathrm{s}\end{array}$ & $\begin{array}{c}\text { MRM } \\
\text { Can } \\
\text { Sampled }\end{array}$ & $\begin{array}{l}\text { Total } \\
\text { press }\end{array}$ & Ch 1 & Ch 2 & Ch 3 & Ch 4 & Ch 5 & Ch 6 & Ch 7 & Ch 8 & Ch 9 & Ch 10 & Ch 11 & Ch 12 & $\begin{array}{c}\text { lon } \\
\text { pressure }\end{array}$ \\
\hline $6 / 14 / 2017$ & Station 3 & L1303 & $1.00 \mathrm{E}-05$ & $1.333 \mathrm{E}-08$ & 8.997E-09 & $6.584 \mathrm{E}-06$ & $\begin{array}{c}0.000 E+0 \\
0\end{array}$ & $6.962 \mathrm{E}-06$ & $1.904 \mathrm{E}-10$ & $1.904 \mathrm{E}-10$ & $2.761 \mathrm{E}-09$ & $1.428 \mathrm{E}-10$ & $4.141 \mathrm{E}-09$ & $1.428 \mathrm{E}-10$ & $1.904 \mathrm{E}-10$ & 7.90E-06 \\
\hline $6 / 21 / 2017$ & Station 3 & L1303 & $1.00 E-05$ & $5.641 \mathrm{E}-08$ & 1.127E-08 & $8.563 \mathrm{E}-06$ & $1.571 \mathrm{E}-09$ & $6.719 \mathrm{E}-07$ & $1.428 \mathrm{E}-10$ & $1.904 \mathrm{E}-10$ & $2.237 \mathrm{E}-09$ & $2.856 \mathrm{E}-10$ & $5.522 \mathrm{E}-09$ & $9.521 \mathrm{E}-11$ & $1.904 \mathrm{E}-10$ & $8.00 \mathrm{E}-06$ \\
\hline $6 / 28 / 2017$ & Station 3 & L1303 & $1.10 \mathrm{E}-05$ & $5.332 \mathrm{E}-08$ & $9.331 \mathrm{E}-09$ & $3.250 \mathrm{E}-06$ & $1.047 \mathrm{E}-09$ & $6.241 \mathrm{E}-07$ & $1.904 \mathrm{E}-10$ & $1.428 \mathrm{E}-10$ & $2.237 \mathrm{E}-09$ & $1.428 \mathrm{E}-10$ & 4.379E-09 & $1.428 \mathrm{E}-10$ & $2.856 \mathrm{E}-10$ & $8.30 \mathrm{E}-06$ \\
\hline $6 / 28 / 2017$ & Station 3 & L1303 & $1.00 \mathrm{E}-05$ & $4.732 \mathrm{E}-08$ & $6.855 \mathrm{E}-09$ & $3.291 \mathrm{E}-06$ & $5.236 \mathrm{E}-10$ & $6.576 \mathrm{E}-07$ & $2.380 \mathrm{E}-10$ & $9.521 \mathrm{E}-11$ & $2.380 \mathrm{E}-09$ & $1.904 \mathrm{E}-10$ & $4.522 \mathrm{E}-09$ & $9.521 \mathrm{E}-11$ & $1.428 \mathrm{E}-10$ & $8.30 \mathrm{E}-06$ \\
\hline $7 / 6 / 2017$ & Station 3 & L1303 & 1.00E-05 & $5.570 \mathrm{E}-08$ & $8.912 \mathrm{E}-09$ & $5.481 \mathrm{E}-06$ & 1.237E-09 & $5.912 \mathrm{E}-07$ & $2.380 \mathrm{E}-10$ & $4.760 \mathrm{E}-11$ & 2.142E-09 & $1.904 \mathrm{E}-10$ & $3.808 \mathrm{E}-09$ & 4.760E-11 & $2.380 \mathrm{E}-10$ & $8.10 \mathrm{E}-06$ \\
\hline $7 / 12 / 2017$ & Station 3 & L1303 & 9.90E-06 & $4.598 \mathrm{E}-08$ & $8.278 \mathrm{E}-09$ & $9.883 \mathrm{E}-06$ & $1.142 \mathrm{E}-09$ & $6.737 \mathrm{E}-07$ & $1.428 \mathrm{E}-10$ & $1.428 \mathrm{E}-10$ & $2.427 \mathrm{E}-09$ & $2.856 \mathrm{E}-10$ & 5.427E-09 & $9.521 \mathrm{E}-11$ & $2.380 \mathrm{E}-10$ & $8.10 \mathrm{E}-06$ \\
\hline $7 / 19 / 2017$ & Station 3 & L1303 & $1.00 \mathrm{E}-05$ & $5.398 \mathrm{E}-08$ & $9.397 \mathrm{E}-09$ & $1.121 \mathrm{E}-05$ & $1.713 \mathrm{E}-09$ & $6.959 \mathrm{E}-07$ & $1.904 \mathrm{E}-10$ & $1.904 \mathrm{E}-10$ & $2.427 \mathrm{E}-09$ & $1.428 \mathrm{E}-10$ & $5.379 \mathrm{E}-09$ & $4.760 \mathrm{E}-11$ & $1.428 \mathrm{E}-10$ & $8.20 \mathrm{E}-06$ \\
\hline $7 / 19 / 2017$ & Station 3 & L1303 & 1.00E-05 & $4.598 \mathrm{E}-08$ & 8.597E-09 & $1.149 \mathrm{E}-05$ & $5.712 \mathrm{E}-10$ & $7.146 \mathrm{E}-07$ & $9.521 \mathrm{E}-11$ & $9.521 \mathrm{E}-11$ & $2.475 \mathrm{E}-09$ & $1.428 \mathrm{E}-10$ & $5.808 \mathrm{E}-09$ & $4.760 \mathrm{E}-11$ & $1.904 \mathrm{E}-10$ & $8.20 \mathrm{E}-06$ \\
\hline $7 / 25 / 2017$ & Station 3 & L1303 & $1.00 E-05$ & 4.465E-08 & $7.712 \mathrm{E}-09$ & $1.039 \mathrm{E}-05$ & $5.712 \mathrm{E}-10$ & 6.797E-07 & $1.428 \mathrm{E}-10$ & $1.904 \mathrm{E}-10$ & $2.332 \mathrm{E}-09$ & $1.428 \mathrm{E}-10$ & $5.332 \mathrm{E}-09$ & $9.521 \mathrm{E}-11$ & $2.380 \mathrm{E}-10$ & $8.10 \mathrm{E}-06$ \\
\hline $8 / 2 / 2017$ & Station 3 & L1303 & $9.90 \mathrm{E}-06$ & $6.284 \mathrm{E}-08$ & 7.779E-09 & $6.747 \mathrm{E}-06$ & $9.521 \mathrm{E}-10$ & 6.696E-07 & $1.904 \mathrm{E}-10$ & $1.904 \mathrm{E}-10$ & $2.285 \mathrm{E}-09$ & $1.428 \mathrm{E}-10$ & $6.093 \mathrm{E}-09$ & $9.521 \mathrm{E}-11$ & $9.521 \mathrm{E}-11$ & $8.00 \mathrm{E}-06$ \\
\hline 8/9/2017 & Station 3 & L1303 & $9.80 \mathrm{E}-06$ & $5.855 \mathrm{E}-08$ & $8.393 \mathrm{E}-09$ & $7.203 \mathrm{E}-06$ & $1.142 \mathrm{E}-09$ & $7.374 \mathrm{E}-07$ & $1.428 \mathrm{E}-10$ & $1.904 \mathrm{E}-10$ & 2.332E-09 & $1.428 \mathrm{E}-10$ & $6.284 \mathrm{E}-09$ & 4.760E-11 & $1.428 \mathrm{E}-10$ & $8.40 \mathrm{E}-06$ \\
\hline $8 / 16 / 2017$ & Station 3 & L1303 & $1.00 \mathrm{E}-05$ & $5.712 \mathrm{E}-08$ & $8.355 \mathrm{E}-09$ & $7.388 \mathrm{E}-06$ & $9.235 \mathrm{E}-10$ & $7.061 \mathrm{E}-07$ & $1.904 \mathrm{E}-10$ & $1.428 \mathrm{E}-10$ & 2.475E-09 & $9.521 \mathrm{E}-11$ & $6.569 \mathrm{E}-09$ & $9.521 \mathrm{E}-11$ & $1.428 \mathrm{E}-10$ & $8.30 \mathrm{E}-06$ \\
\hline $8 / 16 / 2017$ & Station 3 & L1303 & $9.80 \mathrm{E}-06$ & $4.713 \mathrm{E}-08$ & $1.002 \mathrm{E}-08$ & $8.483 \mathrm{E}-06$ & 4.617E-10 & $7.658 \mathrm{E}-07$ & $9.521 \mathrm{E}-11$ & $1.428 \mathrm{E}-10$ & $2.523 \mathrm{E}-09$ & $2.856 \mathrm{E}-10$ & $7.331 \mathrm{E}-09$ & $9.521 \mathrm{E}-11$ & $1.904 \mathrm{E}-10$ & $8.30 \mathrm{E}-06$ \\
\hline $8 / 24 / 2017$ & Station 3 & L1303 & $1.10 \mathrm{E}-05$ & $6.855 \mathrm{E}-08$ & $9.283 \mathrm{E}-09$ & $8.046 \mathrm{E}-06$ & $9.235 \mathrm{E}-10$ & $7.383 \mathrm{E}-07$ & $1.428 \mathrm{E}-10$ & 4.760E-11 & $2.380 \mathrm{E}-09$ & $1.428 \mathrm{E}-10$ & $7.045 \mathrm{E}-09$ & $4.760 \mathrm{E}-11$ & $1.904 \mathrm{E}-10$ & $8.60 \mathrm{E}-06$ \\
\hline $11 / 6 / 2017$ & Station 3 & L1303 & $1.50 \mathrm{E}-05$ & $5.255 \mathrm{E}-07$ & $1.406 \mathrm{E}-06$ & $5.919 \mathrm{E}-06$ & $2.270 \mathrm{E}-09$ & $5.079 \mathrm{E}-07$ & $2.332 \mathrm{E}-09$ & 1.190E-09 & $6.998 \mathrm{E}-09$ & $3.808 \mathrm{E}-10$ & $6.903 \mathrm{E}-09$ & $9.616 \mathrm{E}-09$ & 4.475E-09 & $7.70 \mathrm{E}-06$ \\
\hline $12 / 6 / 2017$ & Station 3 & L1303 & $1.30 \mathrm{E}-05$ & $3.119 \mathrm{E}-07$ & $6.582 \mathrm{E}-08$ & $8.173 \mathrm{E}-06$ & $3.094 \mathrm{E}-09$ & $7.323 \mathrm{E}-07$ & $1.047 \mathrm{E}-09$ & $4.998 \mathrm{E}-09$ & $2.532 \mathrm{E}-08$ & $6.188 \mathrm{E}-10$ & $9.426 \mathrm{E}-09$ & $4.284 \mathrm{E}-10$ & $9.997 \mathrm{E}-10$ & $8.20 \mathrm{E}-06$ \\
\hline $1 / 10 / 2018$ & Station 3 & L1303 & $1.30 \mathrm{E}-05$ & $3.849 \mathrm{E}-07$ & $3.260 \mathrm{E}-08$ & 8.887E-06 & 4.132E-09 & 8.079E-07 & $1.237 \mathrm{E}-09$ & $5.760 \mathrm{E}-09$ & $2.961 \mathrm{E}-08$ & $5.236 \mathrm{E}-10$ & $1.009 \mathrm{E}-08$ & $1.904 \mathrm{E}-10$ & $1.047 \mathrm{E}-09$ & $8.80 \mathrm{E}-06$ \\
\hline $2 / 7 / 2018$ & Station 3 & L1303 & $1.40 \mathrm{E}-05$ & $3.335 \mathrm{E}-07$ & $2.844 \mathrm{E}-08$ & $8.874 \mathrm{E}-06$ & $3.142 \mathrm{E}-09$ & $8.966 \mathrm{E}-07$ & 1.190E-09 & $5.522 \mathrm{E}-09$ & $2.865 \mathrm{E}-08$ & $5.236 \mathrm{E}-10$ & $1.147 \mathrm{E}-08$ & $2.856 \mathrm{E}-10$ & $9.045 \mathrm{E}-10$ & 9.40E-06 \\
\hline $3 / 5 / 2018$ & Station 3 & L1303 & $1.70 \mathrm{E}-05$ & $2.747 \mathrm{E}-07$ & $1.532 \mathrm{E}-08$ & $6.773 \mathrm{E}-06$ & $1.142 \mathrm{E}-09$ & $6.902 \mathrm{E}-07$ & $7.141 \mathrm{E}-10$ & $1.285 \mathrm{E}-09$ & $8.140 \mathrm{E}-09$ & 4.284E-10 & $8.424 \mathrm{E}-09$ & $1.428 \mathrm{E}-10$ & $3.808 \mathrm{E}-10$ & $8.80 \mathrm{E}-06$ \\
\hline $4 / 4 / 2018$ & Station 27 & L1303 & $2.20 \mathrm{E}-05$ & $2.442 \mathrm{E}-07$ & $1.002 \mathrm{E}-08$ & $7.150 \mathrm{E}-06$ & $9.283 \mathrm{E}-10$ & $7.428 \mathrm{E}-07$ & $1.904 \mathrm{E}-10$ & $3.332 \mathrm{E}-10$ & $3.713 \mathrm{E}-09$ & 3.332E-10 & $6.712 \mathrm{E}-09$ & 4.760E-11 & $2.380 \mathrm{E}-10$ & 1.50E-05 \\
\hline $5 / 2 / 2018$ & Station 27 & L1303 & $2.20 \mathrm{E}-05$ & $4.021 \mathrm{E}-07$ & $1.618 \mathrm{E}-08$ & $9.959 \mathrm{E}-06$ & 1.199E-09 & $9.640 \mathrm{E}-07$ & $1.904 \mathrm{E}-10$ & $7.141 \mathrm{E}-10$ & $5.332 \mathrm{E}-09$ & $5.712 \mathrm{E}-10$ & $7.807 \mathrm{E}-09$ & 4.760E-11 & $2.856 \mathrm{E}-10$ & 1.30E-05 \\
\hline $6 / 6 / 2018$ & Station 27 & L1303 & $3.00 \mathrm{E}-05$ & $3.470 \mathrm{E}-07$ & $9.064 \mathrm{E}-09$ & $8.324 \mathrm{E}-06$ & $6.855 \mathrm{E}-10$ & $6.230 \mathrm{E}-07$ & $2.380 \mathrm{E}-10$ & $2.856 \mathrm{E}-10$ & $2.523 \mathrm{E}-09$ & $3.808 \mathrm{E}-10$ & $8.426 \mathrm{E}-09$ & 4.760E-11 & $1.904 \mathrm{E}-10$ & 1.30E-05 \\
\hline $7 / 11 / 2018$ & Station 27 & L1303 & 2.40E-05 & 4.755E-07 & $1.162 \mathrm{E}-08$ & $9.149 \mathrm{E}-06$ & 1.142E-09 & $6.642 \mathrm{E}-07$ & $2.380 \mathrm{E}-10$ & $1.094 \mathrm{E}-09$ & $6.617 \mathrm{E}-09$ & $3.808 \mathrm{E}-10$ & $9.140 \mathrm{E}-09$ & 4.760E-11 & $2.856 \mathrm{E}-10$ & 1.30E-05 \\
\hline $8 / 7 / 2018$ & Station 27 & L1303 & $1.90 \mathrm{E}-05$ & $3.288 \mathrm{E}-07$ & $1.256 \mathrm{E}-08$ & $1.031 \mathrm{E}-05$ & $7.426 \mathrm{E}-10$ & 6.997E-07 & $2.380 \mathrm{E}-10$ & $9.990 \mathrm{E}-10$ & $5.665 \mathrm{E}-09$ & 4.284E-10 & $9.664 \mathrm{E}-09$ & 4.760E-11 & $2.856 \mathrm{E}-10$ & $1.30 \mathrm{E}-05$ \\
\hline $9 / 6 / 2018$ & Station 27 & L1303 & $2.10 \mathrm{E}-05$ & $3.016 \mathrm{E}-07$ & 1.159E-08 & $8.810 \mathrm{E}-06$ & 4.760E-10 & $8.560 \mathrm{E}-07$ & 4.760E-11 & $1.428 \mathrm{E}-10$ & $1.904 \mathrm{E}-09$ & $5.236 \mathrm{E}-10$ & $9.331 \mathrm{E}-09$ & $9.521 \mathrm{E}-11$ & $1.904 \mathrm{E}-10$ & $1.40 \mathrm{E}-05$ \\
\hline $10 / 4 / 2018$ & Station 27 & L1303 & $2.20 \mathrm{E}-05$ & $4.431 \mathrm{E}-07$ & 1.649E-08 & 1.157E-05 & $1.094 \mathrm{E}-09$ & $1.048 \mathrm{E}-06$ & $1.904 \mathrm{E}-10$ & $6.665 \mathrm{E}-10$ & $5.474 \mathrm{E}-09$ & $7.617 \mathrm{E}-10$ & $1.452 \mathrm{E}-08$ & $9.521 \mathrm{E}-11$ & $2.856 \mathrm{E}-10$ & 1.40E-05 \\
\hline $11 / 12 / 2018$ & Station 27 & L1303 & $1.80 \mathrm{E}-05$ & 5.613E-07 & $1.523 \mathrm{E}-08$ & $1.188 \mathrm{E}-05$ & $1.228 \mathrm{E}-09$ & $1.027 \mathrm{E}-06$ & $1.904 \mathrm{E}-10$ & 9.997E-10 & $6.855 \mathrm{E}-09$ & $7.141 \mathrm{E}-10$ & $1.437 \mathrm{E}-08$ & 4.760E-11 & $2.856 \mathrm{E}-10$ & $1.30 \mathrm{E}-05$ \\
\hline $12 / 12 / 2018$ & Station 27 & L1303 & 2.40E-05 & 4.049E-07 & $1.534 \mathrm{E}-08$ & $1.062 \mathrm{E}-05$ & $5.855 \mathrm{E}-10$ & $8.333 \mathrm{E}-07$ & $2.380 \mathrm{E}-10$ & $9.045 \mathrm{E}-10$ & $6.188 \mathrm{E}-09$ & $7.141 \mathrm{E}-10$ & $1.356 \mathrm{E}-08$ & $1.428 \mathrm{E}-10$ & 3.332E-10 & $1.20 \mathrm{E}-05$ \\
\hline $1 / 9 / 2019$ & Station 27 & L1303 & 2.00E-05 & $2.467 \mathrm{E}-07$ & $1.299 \mathrm{E}-08$ & $8.280 \mathrm{E}-06$ & $6.855 \mathrm{E}-10$ & 8.217E-07 & $1.428 \mathrm{E}-10$ & $4.760 \mathrm{E}-10$ & $3.808 \mathrm{E}-09$ & $6.188 \mathrm{E}-10$ & $1.142 \mathrm{E}-08$ & $9.521 \mathrm{E}-11$ & $3.332 \mathrm{E}-10$ & $1.20 \mathrm{E}-05$ \\
\hline
\end{tabular}




\begin{tabular}{|c|c|c|c|c|c|c|c|c|c|c|c|c|c|c|c|c|}
\hline Date & $\begin{array}{l}\text { Comment } \\
\mathrm{s}\end{array}$ & $\begin{array}{c}\text { MRM } \\
\text { Can } \\
\text { Sampled }\end{array}$ & $\begin{array}{l}\text { Total } \\
\text { press }\end{array}$ & Ch 1 & Ch 2 & Ch 3 & Ch 4 & Ch 5 & Ch 6 & Ch 7 & Ch 8 & Ch 9 & Ch 10 & Ch 11 & Ch 12 & $\begin{array}{c}\text { Ion } \\
\text { pressure }\end{array}$ \\
\hline $9 / 20 / 2018$ & Station 13 & L1306 & $2.10 \mathrm{E}-05$ & $1.249 \mathrm{E}-07$ & $2.784 \mathrm{E}-08$ & $1.277 \mathrm{E}-05$ & $2.570 \mathrm{E}-09$ & $1.201 \mathrm{E}-06$ & 4.760E-10 & $2.189 \mathrm{E}-09$ & $1.333 \mathrm{E}-08$ & $7.141 \mathrm{E}-10$ & $1.466 \mathrm{E}-08$ & $9.521 \mathrm{E}-11$ & $5.236 \mathrm{E}-10$ & $1.30 \mathrm{E}-05$ \\
\hline $9 / 27 / 2018$ & Station 13 & L1306 & $1.70 \mathrm{E}-05$ & $1.281 \mathrm{E}-07$ & $3.149 \mathrm{E}-08$ & 1.533E-05 & $3.427 \mathrm{E}-09$ & $1.319 \mathrm{E}-06$ & $7.141 \mathrm{E}-10$ & $3.237 \mathrm{E}-09$ & $1.813 \mathrm{E}-08$ & 8.093E-10 & $1.618 \mathrm{E}-08$ & $1.428 \mathrm{E}-10$ & $6.188 \mathrm{E}-10$ & $1.30 \mathrm{E}-05$ \\
\hline $10 / 4 / 2018$ & Station 13 & L1306 & 2.40E-05 & $1.281 \mathrm{E}-07$ & $2.499 \mathrm{E}-08$ & $1.464 \mathrm{E}-05$ & 3.503E-09 & $1.433 \mathrm{E}-06$ & $5.712 \mathrm{E}-10$ & $3.380 \mathrm{E}-09$ & $1.951 \mathrm{E}-08$ & $5.236 \mathrm{E}-10$ & $1.775 \mathrm{E}-08$ & $1.904 \mathrm{E}-10$ & $5.712 \mathrm{E}-10$ & $1.50 \mathrm{E}-05$ \\
\hline $10 / 11 / 2018$ & Station 13 & L1306 & $2.40 \mathrm{E}-05$ & $1.571 \mathrm{E}-07$ & $2.849 \mathrm{E}-08$ & $1.477 \mathrm{E}-05$ & $3.132 \mathrm{E}-09$ & $1.350 \mathrm{E}-06$ & $5.236 \mathrm{E}-10$ & $3.332 \mathrm{E}-09$ & $1.851 \mathrm{E}-08$ & $9.045 \mathrm{E}-10$ & $1.737 \mathrm{E}-08$ & $1.904 \mathrm{E}-10$ & $5.712 \mathrm{E}-10$ & $1.50 \mathrm{E}-05$ \\
\hline $11 / 13 / 2018$ & Station 13 & L1306 & $2.10 \mathrm{E}-05$ & $3.445 \mathrm{E}-07$ & $2.199 \mathrm{E}-08$ & $8.594 \mathrm{E}-06$ & $4.094 \mathrm{E}-10$ & 7.315E-07 & $1.904 \mathrm{E}-10$ & $2.856 \mathrm{E}-10$ & $2.713 \mathrm{E}-09$ & $5.712 \mathrm{E}-10$ & $9.569 \mathrm{E}-09$ & $1.428 \mathrm{E}-10$ & $2.856 \mathrm{E}-10$ & $1.30 \mathrm{E}-05$ \\
\hline $12 / 12 / 2018$ & Station 13 & L1306 & $2.20 \mathrm{E}-05$ & $5.258 \mathrm{E}-07$ & $3.394 \mathrm{E}-08$ & $1.276 \mathrm{E}-05$ & $2.732 \mathrm{E}-09$ & $1.001 \mathrm{E}-06$ & 4.760E-10 & $2.666 \mathrm{E}-09$ & $1.542 \mathrm{E}-08$ & 7.617E-10 & $1.613 \mathrm{E}-08$ & $1.428 \mathrm{E}-10$ & $5.712 \mathrm{E}-10$ & $1.30 \mathrm{E}-05$ \\
\hline $1 / 9 / 2019$ & Station 13 & L1306 & $1.90 \mathrm{E}-05$ & $2.89 \mathrm{E}-07$ & $1.956 \mathrm{E}-08$ & $1.060 \mathrm{E}-05$ & $1.028 \mathrm{E}-09$ & $1.043 \mathrm{E}-06$ & $2.856 \mathrm{E}-10$ & $8.093 \mathrm{E}-10$ & $5.998 \mathrm{E}-09$ & $9.045 \mathrm{E}-10$ & 1.447E-08 & $1.904 \mathrm{E}-10$ & $3.808 \mathrm{E}-10$ & $1.30 \mathrm{E}-05$ \\
\hline $12 / 22 / 2014$ & Station 11 & L1307 & $1.00 \mathrm{E}-05$ & 8.397E-08 & $1.582 \mathrm{E}-08$ & $6.674 \mathrm{E}-06$ & $9.902 \mathrm{E}-10$ & $4.941 \mathrm{E}-07$ & $8.093 \mathrm{E}-10$ & $2.380 \mathrm{E}-10$ & $2.951 \mathrm{E}-09$ & $6.665 \mathrm{E}-10$ & $8.950 \mathrm{E}-09$ & $\begin{array}{c}0.000 E+0 \\
0\end{array}$ & $\begin{array}{c}0.000 E+0 \\
0\end{array}$ & $6.00 \mathrm{E}-06$ \\
\hline $12 / 22 / 2014$ & Station 11 & L1307 & $9.30 \mathrm{E}-06$ & 7.604E-08 & $1.011 \mathrm{E}-08$ & $6.246 \mathrm{E}-06$ & $1.485 \mathrm{E}-09$ & $4.958 \mathrm{E}-07$ & $9.045 \mathrm{E}-10$ & $2.380 \mathrm{E}-10$ & $2.856 \mathrm{E}-09$ & 3.332E-10 & $8.759 \mathrm{E}-09$ & $\begin{array}{c}0.000 E+0 \\
0\end{array}$ & $\begin{array}{c}0.000 E+0 \\
0\end{array}$ & $6.00 \mathrm{E}-06$ \\
\hline $1 / 7 / 2015$ & Station 11 & L1307 & $1.00 \mathrm{E}-05$ & $2.024 \mathrm{E}-07$ & $1.828 \mathrm{E}-08$ & $5.430 \mathrm{E}-06$ & $2.970 \mathrm{E}-09$ & $4.290 \mathrm{E}-07$ & 4.570E-09 & $3.040 \mathrm{E}-09$ & $1.460 \mathrm{E}-08$ & $5.713 \mathrm{E}-10$ & $6.760 \mathrm{E}-09$ & $\begin{array}{c}0.000 E+0 \\
0 \\
\end{array}$ & $\begin{array}{c}0.000 E+0 \\
0 \\
\end{array}$ & $6.10 \mathrm{E}-06$ \\
\hline $1 / 7 / 2015$ & Station 11 & L1307 & $1.10 \mathrm{E}-05$ & $1.842 \mathrm{E}-07$ & $1.526 \mathrm{E}-08$ & $5.239 \mathrm{E}-06$ & $2.970 \mathrm{E}-09$ & $4.320 \mathrm{E}-07$ & $4.475 \mathrm{E}-09$ & $2.904 \mathrm{E}-09$ & $1.397 \mathrm{E}-08$ & $4.760 \mathrm{E}-10$ & $6.613 \mathrm{E}-09$ & $\begin{array}{c}0.000 \mathrm{E}+0 \\
0\end{array}$ & $\begin{array}{c}0.000 \mathrm{E}+0 \\
0\end{array}$ & $6.10 \mathrm{E}-06$ \\
\hline $1 / 14 / 2015$ & Station 11 & L1307 & $8.10 \mathrm{E}-06$ & $6.838 \mathrm{E}-08$ & $1.138 \mathrm{E}-08$ & $5.750 \mathrm{E}-06$ & $1.237 \mathrm{E}-09$ & $5.610 \mathrm{E}-07$ & $2.142 \mathrm{E}-09$ & $2.850 \mathrm{E}-10$ & $3.094 \mathrm{E}-09$ & 4.284E-10 & $8.333 \mathrm{E}-09$ & $\begin{array}{c}0.000 \mathrm{E}+0 \\
0\end{array}$ & $\begin{array}{c}0.000 \mathrm{E}+0 \\
0\end{array}$ & $6.10 \mathrm{E}-06$ \\
\hline $1 / 21 / 2015$ & Station 11 & L1307 & $8.00 \mathrm{E}-06$ & $6.069 \mathrm{E}-08$ & $8.707 \mathrm{E}-09$ & $5.703 \mathrm{E}-06$ & $8.569 \mathrm{E}-10$ & 4.703E-07 & $1.094 \mathrm{E}-09$ & $1.423 \mathrm{E}-10$ & $2.333 \mathrm{E}-09$ & $3.808 \mathrm{E}-10$ & $5.998 \mathrm{E}-09$ & $\begin{array}{c}0.000 \mathrm{E}+0 \\
0 \\
\end{array}$ & $\begin{array}{c}0.000 E+0 \\
0 \\
\end{array}$ & $6.30 \mathrm{E}-06$ \\
\hline $1 / 28 / 2015$ & Station 11 & L1307 & $9.10 \mathrm{E}-06$ & $8.879 \mathrm{E}-08$ & $5.590 \mathrm{E}-09$ & $3.400 \mathrm{E}-06$ & $2.375 \mathrm{E}-09$ & & & $3.320 \mathrm{E}-09$ & & $3.000 \mathrm{E}-10$ & & $\begin{array}{c}0.000 \mathrm{E}+0 \\
0 \\
\end{array}$ & $\begin{array}{c}0.000 E+0 \\
0 \\
\end{array}$ & $6.60 \mathrm{E}-06$ \\
\hline $3 / 28 / 2017$ & Station 25 & L1307 & $8.10 \mathrm{E}-06$ & 4.020E-06 & $2.133 \mathrm{E}-07$ & $7.832 \mathrm{E}-06$ & $1.809 \mathrm{E}-09$ & 4.009E-07 & $1.142 \mathrm{E}-09$ & $3.808 \mathrm{E}-10$ & $2.904 \mathrm{E}-09$ & $1.904 \mathrm{E}-10$ & $4.808 \mathrm{E}-09$ & $\begin{array}{c}0.000 \mathrm{E}+0 \\
0\end{array}$ & $\begin{array}{c}0.000 \mathrm{E}+0 \\
0 \\
\end{array}$ & $5.60 \mathrm{E}-06$ \\
\hline 4/27/2017 & Station 25 & L1307 & $1.10 \mathrm{E}-05$ & $6.802 \mathrm{E}-07$ & $1.638 \mathrm{E}-07$ & $5.570 \mathrm{E}-06$ & $1.928 \mathrm{E}-09$ & 4.093E-07 & 7.617E-10 & $3.808 \mathrm{E}-10$ & $2.475 \mathrm{E}-09$ & 4.284E-10 & $5.427 \mathrm{E}-09$ & $1.047 \mathrm{E}-09$ & $2.466 \mathrm{E}-09$ & $6.10 \mathrm{E}-06$ \\
\hline $5 / 25 / 2017$ & Station 25 & L1307 & 7.00E-06 & 7.607E-07 & $1.565 \mathrm{E}-08$ & 4.565E-06 & $6.474 \mathrm{E}-10$ & $5.050 \mathrm{E}-07$ & $5.236 \mathrm{E}-10$ & $1.904 \mathrm{E}-10$ & $1.951 \mathrm{E}-09$ & $3.332 \mathrm{E}-10$ & $5.522 \mathrm{E}-09$ & $2.380 \mathrm{E}-10$ & $2.380 \mathrm{E}-10$ & $6.60 \mathrm{E}-06$ \\
\hline $6 / 21 / 2017$ & Station 25 & L1307 & $1.20 \mathrm{E}-05$ & $4.734 \mathrm{E}-07$ & $1.127 \mathrm{E}-08$ & $5.314 \mathrm{E}-06$ & $5.236 \mathrm{E}-10$ & $5.755 \mathrm{E}-07$ & $1.428 \mathrm{E}-10$ & 4.760E-11 & $2.142 \mathrm{E}-09$ & $1.904 \mathrm{E}-10$ & 4.855E-09 & $4.760 \mathrm{E}-11$ & $1.904 \mathrm{E}-10$ & $7.90 \mathrm{E}-06$ \\
\hline $7 / 25 / 2017$ & Station 25 & L1307 & 1.40E-05 & 4.865E-07 & $9.426 \mathrm{E}-09$ & $5.923 \mathrm{E}-06$ & $1.713 \mathrm{E}-09$ & $5.370 \mathrm{E}-07$ & $9.521 \mathrm{E}-11$ & $1.428 \mathrm{E}-10$ & $1.809 \mathrm{E}-09$ & $2.380 \mathrm{E}-10$ & 4.475E-09 & $1.428 \mathrm{E}-10$ & $9.521 \mathrm{E}-11$ & $8.00 \mathrm{E}-06$ \\
\hline $8 / 24 / 2017$ & Station 25 & L1307 & $1.50 \mathrm{E}-05$ & $4.348 \mathrm{E}-07$ & $1.114 \mathrm{E}-08$ & $6.350 \mathrm{E}-06$ & $9.235 \mathrm{E}-10$ & $5.702 \mathrm{E}-07$ & $4.760 \mathrm{E}-11$ & $9.521 \mathrm{E}-11$ & $1.904 \mathrm{E}-09$ & $1.904 \mathrm{E}-10$ & $5.236 \mathrm{E}-09$ & $4.760 \mathrm{E}-11$ & $2.380 \mathrm{E}-10$ & $8.40 \mathrm{E}-06$ \\
\hline $11 / 6 / 2017$ & Station 25 & L1307 & 1.40E-05 & $1.443 \mathrm{E}-06$ & $3.959 \mathrm{E}-08$ & $6.672 \mathrm{E}-06$ & $1.261 \mathrm{E}-09$ & $5.770 \mathrm{E}-07$ & 4.284E-10 & $7.141 \mathrm{E}-10$ & $5.427 \mathrm{E}-09$ & $3.808 \mathrm{E}-10$ & $7.950 \mathrm{E}-09$ & $2.856 \mathrm{E}-10$ & $5.236 \mathrm{E}-10$ & $7.70 \mathrm{E}-06$ \\
\hline $1 / 10 / 2018$ & Station 25 & L1307 & $1.50 \mathrm{E}-05$ & $1.252 \mathrm{E}-06$ & 4.676E-08 & 7.356E-06 & $1.770 \mathrm{E}-09$ & $6.792 \mathrm{E}-07$ & $6.665 \mathrm{E}-10$ & $1.571 \mathrm{E}-09$ & $9.283 \mathrm{E}-09$ & $3.808 \mathrm{E}-10$ & $8.188 \mathrm{E}-09$ & 3.332E-10 & $5.712 \mathrm{E}-10$ & $8.60 \mathrm{E}-06$ \\
\hline $3 / 5 / 2018$ & Station 25 & L1307 & $1.70 \mathrm{E}-05$ & $9.896 \mathrm{E}-07$ & 3.589E-08 & $7.085 \mathrm{E}-06$ & $2.285 \mathrm{E}-09$ & $7.002 E-07$ & 7.617E-10 & 2.047E-09 & $1.185 \mathrm{E}-08$ & $5.712 \mathrm{E}-10$ & $8.902 E-09$ & $1.904 \mathrm{E}-10$ & $6.188 \mathrm{E}-10$ & $8.70 \mathrm{E}-06$ \\
\hline $4 / 4 / 2018$ & Station 25 & L1307 & $2.20 \mathrm{E}-05$ & $5.304 \mathrm{E}-07$ & $1.782 \mathrm{E}-08$ & $6.997 \mathrm{E}-06$ & $6.188 \mathrm{E}-10$ & $7.211 \mathrm{E}-07$ & $1.428 \mathrm{E}-10$ & $4.760 \mathrm{E}-10$ & $3.713 \mathrm{E}-09$ & 3.332E-10 & $6.522 \mathrm{E}-09$ & $1.428 \mathrm{E}-10$ & $2.380 \mathrm{E}-10$ & $1.40 \mathrm{E}-05$ \\
\hline $5 / 2 / 2018$ & Station 25 & L1307 & 2.30E-05 & $6.692 \mathrm{E}-07$ & $2.113 \mathrm{E}-08$ & $1.072 \mathrm{E}-05$ & $8.997 \mathrm{E}-10$ & $9.597 \mathrm{E}-07$ & $3.332 \mathrm{E}-10$ & $9.997 \mathrm{E}-10$ & $5.903 \mathrm{E}-09$ & $6.188 \mathrm{E}-10$ & $8.236 \mathrm{E}-09$ & $1.428 \mathrm{E}-10$ & $2.856 \mathrm{E}-10$ & $1.20 \mathrm{E}-05$ \\
\hline $6 / 6 / 2018$ & Station 25 & L1307 & 2.90E-05 & $4.953 \mathrm{E}-07$ & $9.064 \mathrm{E}-09$ & $9.313 \mathrm{E}-06$ & $6.855 \mathrm{E}-10$ & $6.652 \mathrm{E}-07$ & $1.428 \mathrm{E}-10$ & $2.380 \mathrm{E}-10$ & $2.904 \mathrm{E}-09$ & $2.856 \mathrm{E}-10$ & $9.569 \mathrm{E}-09$ & $4.760 \mathrm{E}-11$ & $2.380 \mathrm{E}-10$ & $1.20 \mathrm{E}-05$ \\
\hline $7 / 11 / 2018$ & Station 25 & L1307 & $2.60 \mathrm{E}-05$ & $8.337 \mathrm{E}-07$ & $2.078 \mathrm{E}-08$ & $1.081 \mathrm{E}-05$ & $1.828 \mathrm{E}-09$ & $7.890 \mathrm{E}-07$ & $4.284 \mathrm{E}-10$ & $2.475 \mathrm{E}-09$ & $1.323 \mathrm{E}-08$ & $5.236 \mathrm{E}-10$ & $1.104 \mathrm{E}-08$ & $9.521 \mathrm{E}-11$ & $4.760 \mathrm{E}-10$ & $1.30 \mathrm{E}-05$ \\
\hline
\end{tabular}




\begin{tabular}{|c|c|c|c|c|c|c|c|c|c|c|c|c|c|c|c|c|}
\hline Date & $\begin{array}{l}\text { Comment } \\
\mathrm{s}\end{array}$ & $\begin{array}{c}\text { MRM } \\
\text { Can } \\
\text { Sampled }\end{array}$ & $\begin{array}{l}\text { Total } \\
\text { press }\end{array}$ & Ch 1 & Ch 2 & Ch 3 & Ch 4 & Ch 5 & Ch 6 & $\mathrm{Ch} 7$ & Ch 8 & Ch 9 & Ch 10 & Ch 11 & Ch 12 & $\begin{array}{c}\text { Ion } \\
\text { pressure }\end{array}$ \\
\hline $8 / 7 / 2018$ & Station 25 & L1307 & $2.10 \mathrm{E}-05$ & $5.802 \mathrm{E}-07$ & $1.542 \mathrm{E}-08$ & $1.056 \mathrm{E}-05$ & $1.237 \mathrm{E}-09$ & $7.379 \mathrm{E}-07$ & $2.380 \mathrm{E}-10$ & $1.285 \mathrm{E}-09$ & $7.093 \mathrm{E}-09$ & $5.236 \mathrm{E}-10$ & $9.854 \mathrm{E}-09$ & $9.521 \mathrm{E}-11$ & $3.808 \mathrm{E}-10$ & $1.30 \mathrm{E}-05$ \\
\hline 9/6/2018 & Station 25 & L1307 & $2.20 \mathrm{E}-05$ & 4.833E-07 & $1.219 \mathrm{E}-08$ & $8.208 \mathrm{E}-06$ & $2.380 \mathrm{E}-10$ & $8.313 \mathrm{E}-07$ & $9.521 \mathrm{E}-11$ & $1.428 \mathrm{E}-10$ & $1.904 \mathrm{E}-09$ & $4.284 \mathrm{E}-10$ & $9.238 \mathrm{E}-09$ & $4.760 \mathrm{E}-11$ & $1.428 \mathrm{E}-10$ & 1.40E-05 \\
\hline $10 / 4 / 2018$ & Station 25 & L1307 & $2.20 \mathrm{E}-05$ & $6.974 \mathrm{E}-07$ & $1.766 \mathrm{E}-08$ & 1.207E-05 & $1.094 \mathrm{E}-09$ & $1.090 \mathrm{E}-06$ & $2.380 \mathrm{E}-10$ & $7.617 \mathrm{E}-10$ & $6.093 \mathrm{E}-09$ & $7.141 \mathrm{E}-10$ & $1.490 \mathrm{E}-08$ & $4.760 \mathrm{E}-11$ & $2.856 \mathrm{E}-10$ & 1.40E-05 \\
\hline $11 / 12 / 2018$ & Station 25 & L1307 & $1.90 \mathrm{E}-05$ & $9.352 \mathrm{E}-07$ & $1.869 \mathrm{E}-08$ & $1.245 \mathrm{E}-05$ & $1.228 \mathrm{E}-09$ & $1.109 \mathrm{E}-06$ & $3.808 \mathrm{E}-10$ & $1.380 \mathrm{E}-09$ & $8.902 \mathrm{E}-09$ & $6.188 \mathrm{E}-10$ & $1.528 \mathrm{E}-08$ & $1.428 \mathrm{E}-10$ & $3.332 \mathrm{E}-10$ & 1.30E-05 \\
\hline $12 / 12 / 2018$ & Station 25 & L1307 & $2.20 \mathrm{E}-05$ & $6.478 \mathrm{E}-07$ & $1.505 \mathrm{E}-08$ & 1.117E-05 & $9.759 \mathrm{E}-10$ & $8.835 \mathrm{E}-07$ & $2.380 \mathrm{E}-10$ & $1.047 \mathrm{E}-09$ & $7.141 \mathrm{E}-09$ & $8.093 \mathrm{E}-10$ & $1.413 \mathrm{E}-08$ & $9.521 \mathrm{E}-11$ & $3.808 \mathrm{E}-10$ & 1.30E-05 \\
\hline $1 / 9 / 2019$ & Station 25 & L1307 & $1.90 \mathrm{E}-05$ & $3.869 \mathrm{E}-07$ & 1.356E-08 & $9.266 \mathrm{E}-06$ & $8.569 \mathrm{E}-10$ & $9.077 \mathrm{E}-07$ & $1.904 \mathrm{E}-10$ & $6.188 \mathrm{E}-10$ & 4.522E-09 & $8.093 \mathrm{E}-10$ & $1.252 \mathrm{E}-08$ & $1.428 \mathrm{E}-10$ & $2.856 \mathrm{E}-10$ & $1.20 \mathrm{E}-05$ \\
\hline $12 / 10 / 2014$ & Man B & $\begin{array}{l}\text { L1307, } \\
\text { L1308 }\end{array}$ & $6.70 \mathrm{E}-06$ & $1.488 \mathrm{E}-06$ & $8.899 \mathrm{E}-08$ & $6.037 \mathrm{E}-06$ & $3.199 \mathrm{E}-09$ & $5.415 \mathrm{E}-07$ & $1.285 \mathrm{E}-09$ & $5.712 \mathrm{E}-10$ & $3.522 \mathrm{E}-09$ & $4.284 \mathrm{E}-10$ & $7.712 \mathrm{E}-09$ & $\begin{array}{c}0.000 \mathrm{E}+0 \\
0\end{array}$ & $\begin{array}{c}0.000 \mathrm{E}+0 \\
0\end{array}$ & $5.70 \mathrm{E}-06$ \\
\hline $12 / 10 / 2014$ & Man B & $\begin{array}{l}\text { L1307, } \\
\text { L1308 }\end{array}$ & $6.30 \mathrm{E}-06$ & $1.371 \mathrm{E}-06$ & $1.008 \mathrm{E}-07$ & $6.249 \mathrm{E}-06$ & $2.285 \mathrm{E}-09$ & $5.406 \mathrm{E}-07$ & $1.285 \mathrm{E}-09$ & $5.712 \mathrm{E}-10$ & $3.522 \mathrm{E}-09$ & $3.808 \mathrm{E}-10$ & 7.331E-09 & $\begin{array}{c}0.000 \mathrm{E}+0 \\
0\end{array}$ & $\begin{array}{c}0.000 \mathrm{E}+0 \\
0\end{array}$ & $5.80 \mathrm{E}-06$ \\
\hline $12 / 17 / 2014$ & Man B & $\begin{array}{l}\text { L1307, } \\
\text { L1308 }\end{array}$ & $9.50 \mathrm{E}-06$ & $1.337 \mathrm{E}-07$ & $1.225 \mathrm{E}-08$ & $5.248 \mathrm{E}-06$ & $7.426 \mathrm{E}-10$ & $5.368 \mathrm{E}-07$ & $9.997 \mathrm{E}-10$ & $2.380 \mathrm{E}-10$ & $2.523 \mathrm{E}-09$ & $4.284 \mathrm{E}-10$ & $6.617 \mathrm{E}-09$ & $\begin{array}{c}0.000 \mathrm{E}+0 \\
0\end{array}$ & $\begin{array}{c}0.000 \mathrm{E}+0 \\
0\end{array}$ & $6.00 \mathrm{E}-06$ \\
\hline $12 / 17 / 2014$ & Man B & $\begin{array}{l}\text { L1307, } \\
\text { L1308 }\end{array}$ & $8.40 \mathrm{E}-06$ & $1.256 \mathrm{E}-07$ & $1.250 \mathrm{E}-08$ & $5.692 \mathrm{E}-06$ & $9.902 \mathrm{E}-10$ & $5.271 \mathrm{E}-07$ & $9.521 \mathrm{E}-10$ & $3.332 \mathrm{E}-10$ & $2.618 \mathrm{E}-09$ & $4.760 \mathrm{E}-10$ & $6.950 \mathrm{E}-09$ & $\begin{array}{c}0.000 \mathrm{E}+0 \\
0\end{array}$ & $\begin{array}{c}0.000 \mathrm{E}+0 \\
0\end{array}$ & $6.00 \mathrm{E}-06$ \\
\hline $12 / 22 / 2014$ & Station 12 & L1308 & $9.30 \mathrm{E}-06$ & $6.298 \mathrm{E}-08$ & $1.475 \mathrm{E}-08$ & $6.345 \mathrm{E}-06$ & $1.237 \mathrm{E}-09$ & $4.785 \mathrm{E}-07$ & $7.141 \mathrm{E}-10$ & $3.330 \mathrm{E}-10$ & $2.713 \mathrm{E}-09$ & $4.234 \mathrm{E}-10$ & $8.569 \mathrm{E}-09$ & $\begin{array}{c}0.000 \mathrm{E}+0 \\
0 \\
\end{array}$ & $\begin{array}{c}0.000 \mathrm{E}+0 \\
0 \\
\end{array}$ & $6.00 \mathrm{E}-06$ \\
\hline $1 / 7 / 2015$ & Station 12 & L1308 & $1.20 \mathrm{E}-05$ & $1.650 \mathrm{E}-07$ & $1.413 \mathrm{E}-07$ & $6.240 \mathrm{E}-06$ & $3.200 \mathrm{E}-09$ & 4.000E-07 & 4.570E-09 & $2.710 \mathrm{E}-09$ & $8.470 \mathrm{E}-09$ & $3.520 \mathrm{E}-09$ & $6.330 \mathrm{E}-09$ & $\begin{array}{c}0.000 \mathrm{E}+0 \\
0 \\
\end{array}$ & $\begin{array}{c}0.000 \mathrm{E}+0 \\
0 \\
\end{array}$ & $6.00 \mathrm{E}-06$ \\
\hline $1 / 14 / 2015$ & Station 12 & L1308 & $9.20 \mathrm{E}-06$ & $5.989 \mathrm{E}-08$ & 1.697E-08 & $5.323 \mathrm{E}-06$ & $1.237 \mathrm{E}-09$ & 4.956E-07 & $1.428 \mathrm{E}-10$ & $2.380 \mathrm{E}-10$ & $2.713 \mathrm{E}-09$ & $3.880 \mathrm{E}-10$ & $6.950 \mathrm{E}-09$ & $\begin{array}{c}0.000 \mathrm{E}+0 \\
0\end{array}$ & $\begin{array}{c}0.000 \mathrm{E}+0 \\
0\end{array}$ & $6.10 \mathrm{E}-06$ \\
\hline $1 / 21 / 2015$ & Station 12 & L1308 & $9.30 \mathrm{E}-06$ & $5.098 \mathrm{E}-08$ & $1.136 \mathrm{E}-08$ & $5.148 \mathrm{E}-06$ & $8.569 \mathrm{E}-10$ & $4.302 \mathrm{E}-07$ & $8.529 \mathrm{E}-10$ & $2.856 \mathrm{E}-10$ & $2.285 \mathrm{E}-09$ & $2.856 \mathrm{E}-10$ & $5.236 \mathrm{E}-09$ & $\begin{array}{c}0.000 \mathrm{E}+0 \\
0\end{array}$ & $\begin{array}{c}0.000 E+0 \\
0\end{array}$ & $6.30 \mathrm{E}-06$ \\
\hline $1 / 28 / 2015$ & Station 12 & L1308 & $1.10 \mathrm{E}-05$ & $5.879 \mathrm{E}-08$ & $1.254 \mathrm{E}-08$ & $6.226 \mathrm{E}-06$ & $9.283 \mathrm{E}-10$ & 4.951E-07 & 7.517E-10 & $1.428 \mathrm{E}-10$ & 2.237E-09 & $3.308 \mathrm{E}-10$ & $6.089 \mathrm{E}-09$ & $\begin{array}{c}0.000 \mathrm{E}+0 \\
0\end{array}$ & $\begin{array}{c}0.000 E+0 \\
0\end{array}$ & $6.60 \mathrm{E}-06$ \\
\hline $3 / 28 / 2017$ & Station 26 & L1308 & $8.50 \mathrm{E}-06$ & $3.121 \mathrm{E}-06$ & 2.247E-07 & $7.898 \mathrm{E}-06$ & $2.170 \mathrm{E}-09$ & 4.119E- 07 & $1.047 \mathrm{E}-09$ & $5.742 \mathrm{E}-10$ & 2.999E-09 & $6.188 \mathrm{E}-10$ & 4.855E-09 & $\begin{array}{c}0.000 E+0 \\
0\end{array}$ & $\begin{array}{c}0.000 E+0 \\
0\end{array}$ & $5.70 \mathrm{E}-06$ \\
\hline $4 / 27 / 2017$ & Station 26 & L1308 & $1.00 \mathrm{E}-05$ & $6.252 \mathrm{E}-07$ & $2.870 \mathrm{E}-07$ & $5.726 \mathrm{E}-06$ & $1.713 \mathrm{E}-09$ & $3.895 \mathrm{E}-07$ & $6.188 \mathrm{E}-10$ & $3.808 \mathrm{E}-10$ & $2.523 \mathrm{E}-09$ & $3.808 \mathrm{E}-10$ & $5.474 \mathrm{E}-09$ & $1.523 \mathrm{E}-09$ & $2.994 \mathrm{E}-09$ & $6.10 \mathrm{E}-06$ \\
\hline $5 / 25 / 2017$ & Station 26 & L1308 & $7.00 \mathrm{E}-06$ & $8.688 \mathrm{E}-07$ & $2.319 \mathrm{E}-08$ & 4.565E-06 & 4.855E-10 & $5.055 \mathrm{E}-07$ & 4.284E-10 & $1.428 \mathrm{E}-10$ & $1.856 \mathrm{E}-09$ & $3.332 \mathrm{E}-10$ & $5.950 \mathrm{E}-09$ & $2.380 \mathrm{E}-10$ & $3.332 \mathrm{E}-10$ & $6.60 \mathrm{E}-06$ \\
\hline $6 / 21 / 2017$ & Station 26 & L1308 & $1.20 \mathrm{E}-05$ & $5.098 \mathrm{E}-07$ & $1.585 \mathrm{E}-08$ & 4.913E-06 & $1.571 \mathrm{E}-09$ & $5.514 \mathrm{E}-07$ & $1.428 \mathrm{E}-10$ & $1.428 \mathrm{E}-10$ & $1.951 \mathrm{E}-09$ & $1.428 \mathrm{E}-10$ & $4.570 \mathrm{E}-09$ & $9.521 \mathrm{E}-11$ & $2.380 \mathrm{E}-10$ & $7.80 \mathrm{E}-06$ \\
\hline $7 / 25 / 2017$ & Station 26 & L1308 & $1.40 \mathrm{E}-05$ & $5.158 \mathrm{E}-07$ & $1.328 \mathrm{E}-08$ & $6.471 \mathrm{E}-06$ & $5.712 \mathrm{E}-10$ & 5.495E-07 & $1.904 \mathrm{E}-10$ & $9.521 \mathrm{E}-11$ & $1.856 \mathrm{E}-09$ & $1.428 \mathrm{E}-10$ & 4.665E-09 & $9.521 \mathrm{E}-11$ & $9.521 \mathrm{E}-11$ & $8.10 \mathrm{E}-06$ \\
\hline $8 / 24 / 2017$ & Station 26 & L1308 & $1.40 \mathrm{E}-05$ & 4.541E-07 & $1.188 \mathrm{E}-08$ & $6.405 \mathrm{E}-06$ & $9.235 \mathrm{E}-10$ & $5.712 \mathrm{E}-07$ & $9.521 \mathrm{E}-11$ & 4.760E-11 & 1.904E-09 & $1.428 \mathrm{E}-10$ & $5.236 \mathrm{E}-09$ & 4.760E-11 & $1.904 \mathrm{E}-10$ & $8.40 \mathrm{E}-06$ \\
\hline $11 / 6 / 2017$ & Station 26 & L1308 & $1.40 \mathrm{E}-05$ & $1.822 \mathrm{E}-06$ & $1.002 \mathrm{E}-08$ & $6.371 \mathrm{E}-06$ & $7.569 \mathrm{E}-10$ & $5.631 \mathrm{E}-07$ & $3.808 \mathrm{E}-10$ & $8.093 \mathrm{E}-10$ & $5.093 \mathrm{E}-09$ & $2.380 \mathrm{E}-10$ & 7.474E-09 & $9.521 \mathrm{E}-11$ & $3.332 \mathrm{E}-10$ & $7.50 \mathrm{E}-06$ \\
\hline $1 / 10 / 2018$ & Station 26 & L1308 & $1.40 \mathrm{E}-05$ & $1.217 \mathrm{E}-06$ & $1.233 \mathrm{E}-08$ & $6.786 \mathrm{E}-06$ & $1.770 \mathrm{E}-09$ & $6.556 \mathrm{E}-07$ & $3.808 \mathrm{E}-10$ & $1.380 \mathrm{E}-09$ & $8.521 \mathrm{E}-09$ & $4.281 \mathrm{E}-10$ & 7.664E-09 & $9.521 \mathrm{E}-11$ & $4.284 \mathrm{E}-10$ & $8.30 \mathrm{E}-06$ \\
\hline $3 / 5 / 2018$ & Station 26 & L1308 & $1.70 \mathrm{E}-05$ & $9.729 \mathrm{E}-07$ & $1.725 \mathrm{E}-08$ & $7.202 \mathrm{E}-06$ & $1.142 \mathrm{E}-09$ & $7.139 \mathrm{E}-07$ & $6.665 \mathrm{E}-10$ & $1.761 \mathrm{E}-09$ & $1.066 \mathrm{E}-08$ & $4.760 \mathrm{E}-10$ & $8.950 \mathrm{E}-09$ & $1.428 \mathrm{E}-10$ & $5.236 \mathrm{E}-10$ & $8.70 \mathrm{E}-06$ \\
\hline $4 / 4 / 2018$ & Station 26 & L1308 & $2.20 \mathrm{E}-05$ & $5.304 \mathrm{E}-07$ & $9.654 \mathrm{E}-09$ & $7.099 \mathrm{E}-06$ & $3.094 \mathrm{E}-10$ & $7.256 \mathrm{E}-07$ & $1.904 \mathrm{E}-10$ & $3.808 \mathrm{E}-10$ & $3.618 \mathrm{E}-09$ & $3.808 \mathrm{E}-10$ & $6.617 \mathrm{E}-09$ & $1.904 \mathrm{E}-10$ & $1.904 \mathrm{E}-10$ & 1.40E-05 \\
\hline $5 / 2 / 2018$ & Station 26 & L1308 & $2.30 \mathrm{E}-05$ & $6.430 \mathrm{E}-07$ & $1.618 \mathrm{E}-08$ & 9.960E-06 & 1.499E-09 & $9.245 \mathrm{E}-07$ & 2.380E-10 & $8.569 \mathrm{E}-10$ & $5.522 \mathrm{E}-09$ & $5.712 \mathrm{E}-10$ & $7.760 \mathrm{E}-09$ & 4.760E-11 & $3.332 \mathrm{E}-10$ & $1.20 \mathrm{E}-05$ \\
\hline $6 / 6 / 2018$ & Station 26 & L1308 & $3.00 \mathrm{E}-05$ & 5.587E-07 & $9.064 \mathrm{E}-09$ & $8.099 \mathrm{E}-06$ & 4.570E-10 & $6.223 \mathrm{E}-07$ & $1.428 \mathrm{E}-10$ & $2.856 \mathrm{E}-10$ & $2.666 \mathrm{E}-09$ & $3.332 \mathrm{E}-10$ & $8.236 \mathrm{E}-09$ & $9.521 \mathrm{E}-11$ & $2.380 \mathrm{E}-10$ & $1.20 \mathrm{E}-05$ \\
\hline
\end{tabular}




\begin{tabular}{|c|c|c|c|c|c|c|c|c|c|c|c|c|c|c|c|c|}
\hline Date & $\begin{array}{l}\text { Comment } \\
\mathrm{s}\end{array}$ & $\begin{array}{c}\text { MRM } \\
\text { Can } \\
\text { Sampled }\end{array}$ & $\begin{array}{l}\text { Total } \\
\text { press }\end{array}$ & Ch 1 & Ch 2 & Ch 3 & Ch 4 & Ch 5 & Ch 6 & Ch 7 & Ch 8 & Ch 9 & Ch 10 & Ch 11 & Ch 12 & $\begin{array}{c}\text { lon } \\
\text { pressure }\end{array}$ \\
\hline $7 / 11 / 2018$ & Station 26 & L1308 & $2.70 \mathrm{E}-05$ & 7.593E-07 & 1.109E-08 & 8.873E-06 & $1.142 \mathrm{E}-09$ & $6.428 \mathrm{E}-07$ & $2.856 \mathrm{E}-10$ & $1.285 \mathrm{E}-09$ & $7.045 \mathrm{E}-09$ & $3.808 \mathrm{E}-10$ & $8.950 \mathrm{E}-09$ & $4.760 \mathrm{E}-11$ & $3.332 \mathrm{E}-10$ & $1.30 \mathrm{E}-05$ \\
\hline $8 / 7 / 2018$ & Station 26 & L1308 & $2.00 \mathrm{E}-05$ & $5.519 \mathrm{E}-07$ & $1.294 \mathrm{E}-08$ & $1.074 \mathrm{E}-05$ & $9.902 \mathrm{E}-10$ & $7.223 \mathrm{E}-07$ & $1.904 \mathrm{E}-10$ & $9.997 \mathrm{E}-10$ & $6.331 \mathrm{E}-09$ & 4.284E-10 & $9.616 \mathrm{E}-09$ & $9.521 \mathrm{E}-11$ & 2.380E-10 & $1.30 \mathrm{E}-05$ \\
\hline 9/6/2018 & Station 26 & L1308 & 2.10E-05 & $4.558 \mathrm{E}-07$ & 9.597E-09 & $8.024 \mathrm{E}-06$ & $2.380 \mathrm{E}-10$ & $8.057 \mathrm{E}-07$ & $9.521 \mathrm{E}-11$ & $9.521 \mathrm{E}-11$ & $1.904 \mathrm{E}-09$ & $3.332 \mathrm{E}-10$ & $8.759 \mathrm{E}-09$ & 4.760E-11 & $1.428 \mathrm{E}-10$ & 1.40E-05 \\
\hline $10 / 4 / 2018$ & Station 26 & L1308 & $2.20 \mathrm{E}-05$ & $6.821 \mathrm{E}-07$ & $1.616 \mathrm{E}-08$ & $1.162 \mathrm{E}-05$ & $1.094 \mathrm{E}-09$ & $1.056 \mathrm{E}-06$ & $2.856 \mathrm{E}-10$ & $7.141 \mathrm{E}-10$ & $5.570 \mathrm{E}-09$ & $7.141 \mathrm{E}-10$ & $1.442 \mathrm{E}-08$ & $9.521 \mathrm{E}-11$ & $2.856 \mathrm{E}-10$ & 1.40E-05 \\
\hline $11 / 12 / 2018$ & Station 26 & L1308 & $1.90 \mathrm{E}-05$ & $9.122 \mathrm{E}-07$ & $1.555 \mathrm{E}-08$ & $1.208 \mathrm{E}-05$ & $1.228 \mathrm{E}-09$ & $1.064 \mathrm{E}-06$ & $2.380 \mathrm{E}-10$ & $1.142 \mathrm{E}-09$ & 7.617E-09 & $7.141 \mathrm{E}-10$ & $1.475 \mathrm{E}-08$ & $9.521 \mathrm{E}-11$ & $3.332 \mathrm{E}-10$ & $1.30 \mathrm{E}-05$ \\
\hline $12 / 12 / 2018$ & Station 26 & L1308 & $2.40 \mathrm{E}-05$ & $6.310 \mathrm{E}-07$ & $1.460 \mathrm{E}-08$ & $1.050 \mathrm{E}-05$ & $7.800 \mathrm{E}-10$ & $8.380 \mathrm{E}-07$ & $3.330 \mathrm{E}-10$ & $9.040 \mathrm{E}-10$ & $6.520 \mathrm{E}-09$ & 7.610E-10 & $1.340 \mathrm{E}-08$ & $4.760 \mathrm{E}-11$ & $3.330 \mathrm{E}-10$ & 1.20E-05 \\
\hline $1 / 9 / 2019$ & Station 26 & L1308 & $1.90 \mathrm{E}-05$ & $3.922 \mathrm{E}-07$ & $1.299 \mathrm{E}-08$ & $8.703 \mathrm{E}-06$ & $6.855 \mathrm{E}-10$ & $8.603 \mathrm{E}-07$ & $2.380 \mathrm{E}-10$ & $4.284 \mathrm{E}-10$ & $4.141 \mathrm{E}-09$ & $6.665 \mathrm{E}-10$ & 1.185E-08 & $9.521 \mathrm{E}-11$ & $3.332 \mathrm{E}-10$ & $1.20 \mathrm{E}-05$ \\
\hline $9 / 20 / 2018$ & Station 15 & L1311 & $2.00 \mathrm{E}-05$ & $9.097 \mathrm{E}-08$ & $2.152 \mathrm{E}-08$ & $1.281 \mathrm{E}-05$ & $2.142 \mathrm{E}-09$ & 1.199E-06 & $4.284 \mathrm{E}-10$ & $1.856 \mathrm{E}-09$ & $1.118 \mathrm{E}-08$ & $7.141 \mathrm{E}-10$ & $1.461 \mathrm{E}-08$ & $9.521 \mathrm{E}-11$ & $4.284 \mathrm{E}-10$ & $1.30 \mathrm{E}-05$ \\
\hline $9 / 27 / 2018$ & Station 15 & L1311 & $2.10 \mathrm{E}-05$ & $1.029 \mathrm{E}-07$ & $2.599 \mathrm{E}-08$ & $1.429 \mathrm{E}-05$ & $2.785 \mathrm{E}-09$ & $1.269 \mathrm{E}-06$ & $5.236 \mathrm{E}-10$ & $2.475 \mathrm{E}-09$ & $1.432 \mathrm{E}-08$ & $8.569 \mathrm{E}-10$ & $1.609 \mathrm{E}-08$ & $9.521 \mathrm{E}-11$ & $5.236 \mathrm{E}-10$ & 1.30E-05 \\
\hline $10 / 4 / 2018$ & Station 15 & L1311 & $2.30 \mathrm{E}-05$ & $1.138 \mathrm{E}-07$ & $2.366 \mathrm{E}-08$ & $1.476 \mathrm{E}-05$ & 2.627E-09 & $1.394 \mathrm{E}-06$ & $4.760 \mathrm{E}-10$ & $2.713 \mathrm{E}-09$ & $1.594 \mathrm{E}-08$ & $8.093 \mathrm{E}-10$ & $1.756 \mathrm{E}-08$ & $1.428 \mathrm{E}-10$ & $5.236 \mathrm{E}-10$ & 1.50E-05 \\
\hline $10 / 11 / 2018$ & Station 15 & L1311 & $2.50 \mathrm{E}-05$ & $1.452 \mathrm{E}-07$ & $2.749 \mathrm{E}-08$ & $1.532 \mathrm{E}-05$ & $2.685 \mathrm{E}-09$ & 1.363E-06 & $5.712 \mathrm{E}-10$ & $2.713 \mathrm{E}-09$ & $1.628 \mathrm{E}-08$ & $9.521 \mathrm{E}-10$ & $1.809 \mathrm{E}-08$ & $9.521 \mathrm{E}-11$ & $5.712 \mathrm{E}-10$ & 1.50E-05 \\
\hline $11 / 13 / 2018$ & Station 15 & L1311 & $2.00 \mathrm{E}-05$ & $3.330 \mathrm{E}-07$ & $8.640 \mathrm{E}-09$ & $8.674 \mathrm{E}-06$ & $6.141 \mathrm{E}-10$ & $7.401 \mathrm{E}-07$ & $1.428 \mathrm{E}-10$ & $2.380 \mathrm{E}-10$ & $2.666 \mathrm{E}-09$ & $4.284 \mathrm{E}-10$ & $9.711 \mathrm{E}-09$ & $9.521 \mathrm{E}-11$ & $2.380 \mathrm{E}-10$ & $1.30 \mathrm{E}-05$ \\
\hline $12 / 12 / 2018$ & Station 15 & L1311 & $2.20 \mathrm{E}-05$ & $5.098 \mathrm{E}-07$ & $1.992 \mathrm{E}-08$ & $1.280 \mathrm{E}-05$ & $1.951 \mathrm{E}-09$ & $9.905 \mathrm{E}-07$ & $4.760 \mathrm{E}-10$ & $2.237 \mathrm{E}-09$ & $1.342 \mathrm{E}-08$ & $8.093 \mathrm{E}-10$ & $1.585 \mathrm{E}-08$ & $9.521 \mathrm{E}-11$ & $4.760 \mathrm{E}-10$ & $1.30 \mathrm{E}-05$ \\
\hline $1 / 9 / 2019$ & Station 15 & L1311 & $1.80 \mathrm{E}-05$ & $2.765 \mathrm{E}-07$ & $1.528 \mathrm{E}-08$ & $1.007 \mathrm{E}-05$ & $1.028 \mathrm{E}-09$ & $9.968 \mathrm{E}-07$ & $2.380 \mathrm{E}-10$ & $6.665 \mathrm{E}-10$ & $5.379 \mathrm{E}-09$ & 7.617E-10 & $1.371 \mathrm{E}-08$ & 4.760E-11 & $3.332 \mathrm{E}-10$ & $1.20 \mathrm{E}-05$ \\
\hline $6 / 17 / 2014$ & Station 3 & L134 & $8.50 \mathrm{E}-06$ & $6.078 \mathrm{E}-08$ & $1.573 \mathrm{E}-08$ & $6.981 \mathrm{E}-06$ & 4.046E-09 & $5.943 \mathrm{E}-07$ & $3.808 \mathrm{E}-10$ & $1.997 \mathrm{E}-09$ & $1.875 \mathrm{E}-08$ & $3.884 \mathrm{E}-10$ & 4.475E-09 & $\begin{array}{c}0.000 \mathrm{E}+0 \\
0\end{array}$ & $\begin{array}{c}0.000 \mathrm{E}+0 \\
0\end{array}$ & $5.90 \mathrm{E}-06$ \\
\hline $6 / 17 / 2014$ & Station 3 & L134 & $7.30 \mathrm{E}-06$ & $5.558 \mathrm{E}-08$ & $1.330 \mathrm{E}-08$ & $6.799 \mathrm{E}-06$ & $3.237 \mathrm{E}-09$ & $6.296 \mathrm{E}-07$ & $3.618 \mathrm{E}-09$ & $1.999 \mathrm{E}-09$ & $1.071 \mathrm{E}-08$ & $2.380 \mathrm{E}-10$ & 4.475E-09 & $\begin{array}{c}0.000 \mathrm{E}+0 \\
0\end{array}$ & $\begin{array}{c}0.000 E+0 \\
0\end{array}$ & 5.90E-06 \\
\hline $6 / 26 / 2014$ & Station 3 & L134 & $8.50 \mathrm{E}-06$ & $9.397 \mathrm{E}-08$ & $2.262 \mathrm{E}-08$ & $8.475 \mathrm{E}-06$ & 4.046E-09 & $6.395 \mathrm{E}-07$ & $6.141 \mathrm{E}-09$ & 4.427E-09 & $2.247 \mathrm{E}-08$ & $3.808 \mathrm{E}-10$ & 4.332E-09 & $\begin{array}{c}0.000 E+0 \\
0 \\
\end{array}$ & $\begin{array}{c}0.000 E+0 \\
0 \\
\end{array}$ & $5.90 \mathrm{E}-06$ \\
\hline $6 / 26 / 2014$ & Station 3 & L134 & $8.10 \mathrm{E}-06$ & $8.477 \mathrm{E}-08$ & $1.885 \mathrm{E}-08$ & $8.701 \mathrm{E}-06$ & 5.665E-09 & $6.730 \mathrm{E}-07$ & $5.998 \mathrm{E}-09$ & $4.332 \mathrm{E}-09$ & $2.185 \mathrm{E}-08$ & $1.904 \mathrm{E}-10$ & $4.284 \mathrm{E}-09$ & $\begin{array}{c}0.000 \mathrm{E}+0 \\
0\end{array}$ & $\begin{array}{c}0.000 E+0 \\
0\end{array}$ & $5.90 \mathrm{E}-06$ \\
\hline $7 / 2 / 2014$ & Station 3 & L134 & $9.00 \mathrm{E}-06$ & $6.838 \mathrm{E}-08$ & $1.970 \mathrm{E}-08$ & $6.093 \mathrm{E}-06$ & $3.427 \mathrm{E}-09$ & $5.578 \mathrm{E}-07$ & 4.951E-09 & $3.520 \mathrm{E}-09$ & $1.785 \mathrm{E}-08$ & $3.302 \mathrm{E}-10$ & $4.808 \mathrm{E}-09$ & $\begin{array}{c}0.000 \mathrm{E}+0 \\
0\end{array}$ & $\begin{array}{c}0.000 E+0 \\
0\end{array}$ & $6.20 \mathrm{E}-06$ \\
\hline $7 / 2 / 2014$ & Station 3 & L134 & $7.60 \mathrm{E}-06$ & $6.069 \mathrm{E}-08$ & $1.576 \mathrm{E}-08$ & $6.221 \mathrm{E}-06$ & $5.141 \mathrm{E}-09$ & $5.860 \mathrm{E}-07$ & 4.855E-09 & $3.475 \mathrm{E}-09$ & $1.718 \mathrm{E}-08$ & $3.380 \mathrm{E}-10$ & $5.236 \mathrm{E}-09$ & $\begin{array}{c}0.000 E+0 \\
0\end{array}$ & $\begin{array}{c}0.000 E+0 \\
0\end{array}$ & $6.20 \mathrm{E}-06$ \\
\hline $7 / 9 / 2014$ & Station 3 & L134 & $9.30 \mathrm{E}-06$ & $7.369 \mathrm{E}-08$ & $2.055 \mathrm{E}-08$ & $5.201 \mathrm{E}-06$ & $2.499 \mathrm{E}-09$ & $5.818 \mathrm{E}-07$ & $6.046 \mathrm{E}-09$ & 4.617E-09 & $2.308 \mathrm{E}-08$ & $5.236 \mathrm{E}-10$ & $6.141 \mathrm{E}-09$ & $\begin{array}{c}0.000 E+0 \\
0\end{array}$ & $\begin{array}{c}0.000 E+0 \\
0\end{array}$ & $6.00 \mathrm{E}-06$ \\
\hline $7 / 9 / 2014$ & Station 3 & L134 & 7.80E-06 & $6.469 \mathrm{E}-08$ & $1.877 \mathrm{E}-08$ & $7.654 \mathrm{E}-06$ & 2.499E-09 & $6.116 \mathrm{E}-07$ & $5.808 \mathrm{E}-09$ & $4.332 \mathrm{E}-09$ & $2.189 \mathrm{E}-08$ & $4.284 \mathrm{E}-10$ & $6.569 \mathrm{E}-09$ & $\begin{array}{c}0.000 E+0 \\
0\end{array}$ & $\begin{array}{c}0.000 E+0 \\
0\end{array}$ & $6.00 \mathrm{E}-06$ \\
\hline $7 / 16 / 2014$ & Station 3 & L134 & $9.00 \mathrm{E}-06$ & $8.226 \mathrm{E}-08$ & $2.233 \mathrm{E}-08$ & $7.749 \mathrm{E}-06$ & $3.570 \mathrm{E}-09$ & $5.714 \mathrm{E}-07$ & $6.474 \mathrm{E}-09$ & $5.552 \mathrm{E}-09$ & $2.737 \mathrm{E}-08$ & $3.236 \mathrm{E}-10$ & $6.855 \mathrm{E}-09$ & $\begin{array}{c}0.000 E+0 \\
0\end{array}$ & $\begin{array}{c}0.000 E+0 \\
0\end{array}$ & $6.20 \mathrm{E}-06$ \\
\hline $7 / 16 / 2014$ & Station 3 & L134 & $7.90 \mathrm{E}-06$ & $7.241 \mathrm{E}-08$ & $2.039 \mathrm{E}-08$ & $7.772 \mathrm{E}-06$ & 3.927E-09 & $5.746 \mathrm{E}-07$ & $6.141 \mathrm{E}-09$ & $5.046 \mathrm{E}-09$ & $2.518 \mathrm{E}-08$ & $3.808 \mathrm{E}-10$ & $6.807 \mathrm{E}-09$ & $\begin{array}{c}0.000 E+0 \\
0\end{array}$ & $\begin{array}{c}0.000 E+0 \\
0\end{array}$ & $6.20 \mathrm{E}-06$ \\
\hline $3 / 28 / 2017$ & Station 22 & L134 & 9.50E-06 & 4.370E-06 & 7.133E-08 & $6.482 \mathrm{E}-06$ & $1.809 \mathrm{E}-09$ & $3.705 \mathrm{E}-07$ & $8.569 \mathrm{E}-10$ & $4.284 \mathrm{E}-10$ & $2.951 \mathrm{E}-09$ & 7.617E-10 & 4.475E-09 & $\begin{array}{c}0.000 \mathrm{E}+0 \\
0\end{array}$ & $\begin{array}{c}0.000 E+0 \\
0\end{array}$ & 5.30E-06 \\
\hline $4 / 27 / 2017$ & Station 22 & L134 & $1.10 \mathrm{E}-05$ & $1.000 \mathrm{E}-06$ & $3.854 \mathrm{E}-07$ & $5.220 \mathrm{E}-06$ & $1.928 \mathrm{E}-09$ & $3.616 \mathrm{E}-07$ & $6.188 \mathrm{E}-10$ & $3.808 \mathrm{E}-10$ & $2.427 \mathrm{E}-09$ & $3.808 \mathrm{E}-10$ & 4.855E-09 & $2.047 \mathrm{E}-09$ & $3.699 \mathrm{E}-09$ & 5.80E-06 \\
\hline $5 / 25 / 2017$ & Station 22 & L134 & 7.00E-06 & 1.190E-06 & $2.685 \mathrm{E}-08$ & 4.687E-06 & $3.237 \mathrm{E}-10$ & 4.977E-07 & $4.760 \mathrm{E}-10$ & $1.428 \mathrm{E}-10$ & $1.904 \mathrm{E}-09$ & $3.808 \mathrm{E}-10$ & $6.046 \mathrm{E}-09$ & $1.904 \mathrm{E}-10$ & $2.380 \mathrm{E}-10$ & $6.60 \mathrm{E}-06$ \\
\hline
\end{tabular}




\begin{tabular}{|c|c|c|c|c|c|c|c|c|c|c|c|c|c|c|c|c|}
\hline Date & $\begin{array}{l}\text { Comment } \\
\mathrm{s}\end{array}$ & $\begin{array}{c}\text { MRM } \\
\text { Can } \\
\text { Sampled }\end{array}$ & $\begin{array}{l}\text { Total } \\
\text { press }\end{array}$ & Ch 1 & Ch 2 & Ch 3 & Ch 4 & Ch 5 & Ch 6 & $\mathrm{Ch} 7$ & Ch 8 & Ch 9 & Ch 10 & Ch 11 & Ch 12 & $\begin{array}{c}\text { Ion } \\
\text { pressure }\end{array}$ \\
\hline $6 / 21 / 2017$ & Station 22 & L134 & $1.10 \mathrm{E}-05$ & $6.705 \mathrm{E}-07$ & $2.201 \mathrm{E}-08$ & $8.364 \mathrm{E}-06$ & $1.047 \mathrm{E}-09$ & $6.246 \mathrm{E}-07$ & $1.428 \mathrm{E}-10$ & $9.521 \mathrm{E}-11$ & $2.047 \mathrm{E}-09$ & $1.428 \mathrm{E}-10$ & $5.474 \mathrm{E}-09$ & $9.521 \mathrm{E}-11$ & $2.380 \mathrm{E}-10$ & 7.90E-06 \\
\hline $7 / 25 / 2017$ & Station 22 & L134 & $1.40 \mathrm{E}-05$ & $6.385 \mathrm{E}-07$ & $1.306 \mathrm{E}-08$ & $5.832 \mathrm{E}-06$ & $1.142 \mathrm{E}-09$ & 5.417E-07 & $9.521 \mathrm{E}-11$ & $9.521 \mathrm{E}-11$ & $1.904 \mathrm{E}-09$ & $1.904 \mathrm{E}-10$ & $4.522 \mathrm{E}-09$ & $4.760 \mathrm{E}-11$ & $9.521 \mathrm{E}-11$ & $8.10 \mathrm{E}-06$ \\
\hline $8 / 24 / 2017$ & Station 22 & L134 & $1.50 \mathrm{E}-05$ & $5.491 \mathrm{E}-07$ & $1.429 \mathrm{E}-08$ & 7.497E-06 & $9.235 \mathrm{E}-10$ & $6.008 \mathrm{E}-07$ & $9.521 \mathrm{E}-11$ & $9.521 \mathrm{E}-11$ & $1.809 \mathrm{E}-09$ & $2.856 \mathrm{E}-10$ & $5.093 \mathrm{E}-09$ & $1.428 \mathrm{E}-10$ & $2.380 \mathrm{E}-10$ & $8.50 \mathrm{E}-06$ \\
\hline $11 / 6 / 2017$ & Station 22 & L134 & $1.40 \mathrm{E}-05$ & $1.864 \mathrm{E}-06$ & $1.105 \mathrm{E}-08$ & $6.408 \mathrm{E}-06$ & $1.009 \mathrm{E}-09$ & $5.699 \mathrm{E}-07$ & $4.284 \mathrm{E}-10$ & $8.093 \mathrm{E}-10$ & 5.617E-09 & $3.332 \mathrm{E}-10$ & $7.426 \mathrm{E}-09$ & $9.521 \mathrm{E}-11$ & $3.332 \mathrm{E}-10$ & 7.50E-06 \\
\hline $1 / 10 / 2018$ & Station 22 & L134 & $1.50 \mathrm{E}-05$ & $1.322 \mathrm{E}-06$ & $1.416 \mathrm{E}-08$ & 7.137E-06 & $8.854 \mathrm{E}-10$ & $6.618 \mathrm{E}-07$ & $6.665 \mathrm{E}-10$ & $1.761 \mathrm{E}-09$ & $9.997 \mathrm{E}-09$ & $3.808 \mathrm{E}-10$ & $7.950 \mathrm{E}-09$ & $9.521 \mathrm{E}-11$ & 4.284E-10 & $8.40 \mathrm{E}-06$ \\
\hline $3 / 5 / 2018$ & Station 22 & L134 & $1.70 \mathrm{E}-05$ & $1.087 \mathrm{E}-06$ & $2.526 \mathrm{E}-08$ & 8.087E-06 & $3.713 \mathrm{E}-09$ & $7.798 \mathrm{E}-07$ & $9.521 \mathrm{E}-10$ & 4.284E-09 & $2.232 \mathrm{E}-08$ & $6.665 \mathrm{E}-10$ & $1.009 \mathrm{E}-08$ & $1.904 \mathrm{E}-10$ & 9.997E-10 & $8.80 \mathrm{E}-06$ \\
\hline $4 / 4 / 2018$ & Station 22 & L134 & $2.20 \mathrm{E}-05$ & $5.218 \mathrm{E}-07$ & $1.132 \mathrm{E}-08$ & 6.997E-06 & $6.188 \mathrm{E}-10$ & $7.246 \mathrm{E}-07$ & $9.521 \mathrm{E}-11$ & $3.332 \mathrm{E}-10$ & $3.665 \mathrm{E}-09$ & $3.808 \mathrm{E}-10$ & $6.522 \mathrm{E}-09$ & $9.521 \mathrm{E}-11$ & $3.332 \mathrm{E}-10$ & 1.40E-05 \\
\hline $5 / 2 / 2018$ & Station 22 & L134 & $2.10 \mathrm{E}-05$ & $6.493 \mathrm{E}-07$ & $1.713 \mathrm{E}-08$ & $1.116 \mathrm{E}-05$ & 1.199E-09 & $9.787 \mathrm{E}-07$ & $3.808 \mathrm{E}-10$ & $9.521 \mathrm{E}-10$ & $9.331 \mathrm{E}-09$ & $6.188 \mathrm{E}-10$ & $8.712 \mathrm{E}-09$ & $9.521 \mathrm{E}-11$ & $2.856 \mathrm{E}-10$ & $1.20 \mathrm{E}-05$ \\
\hline $6 / 6 / 2018$ & Station 22 & L134 & $2.90 \mathrm{E}-05$ & $6.229 \mathrm{E}-07$ & $1.213 \mathrm{E}-08$ & $1.057 \mathrm{E}-05$ & $6.855 \mathrm{E}-10$ & $7.705 \mathrm{E}-07$ & $2.856 \mathrm{E}-10$ & $3.808 \mathrm{E}-10$ & $3.522 \mathrm{E}-09$ & $5.236 \mathrm{E}-10$ & $1.056 \mathrm{E}-08$ & $1.428 \mathrm{E}-10$ & $2.856 \mathrm{E}-10$ & $1.20 \mathrm{E}-05$ \\
\hline $7 / 11 / 2018$ & Station 22 & L134 & 2.60E-05 & $8.808 \mathrm{E}-07$ & $1.972 \mathrm{E}-08$ & $1.085 \mathrm{E}-05$ & $2.285 \mathrm{E}-09$ & $8.069 \mathrm{E}-07$ & 4.284E-10 & $3.094 \mathrm{E}-09$ & $1.604 \mathrm{E}-08$ & $5.236 \mathrm{E}-10$ & $1.104 \mathrm{E}-08$ & $1.428 \mathrm{E}-10$ & $3.808 \mathrm{E}-10$ & 1.30E-05 \\
\hline $8 / 7 / 2018$ & Station 22 & L134 & $2.20 \mathrm{E}-05$ & $6.074 \mathrm{E}-07$ & $1.352 \mathrm{E}-08$ & $1.056 \mathrm{E}-05$ & $1.237 \mathrm{E}-09$ & $7.428 \mathrm{E}-07$ & $2.856 \mathrm{E}-10$ & $1.285 \mathrm{E}-09$ & $7.664 \mathrm{E}-09$ & $4.760 \mathrm{E}-10$ & $9.759 \mathrm{E}-09$ & $4.760 \mathrm{E}-11$ & $3.332 \mathrm{E}-10$ & $1.30 \mathrm{E}-05$ \\
\hline $9 / 6 / 2018$ & Station 22 & L134 & $2.20 \mathrm{E}-05$ & $5.061 \mathrm{E}-07$ & $1.119 \mathrm{E}-08$ & $8.346 \mathrm{E}-06$ & $2.380 \mathrm{E}-10$ & $8.531 \mathrm{E}-07$ & $9.521 \mathrm{E}-11$ & $9.521 \mathrm{E}-11$ & $1.951 \mathrm{E}-09$ & 4.760E-10 & $9.426 \mathrm{E}-09$ & $4.760 \mathrm{E}-11$ & $1.904 \mathrm{E}-10$ & $1.40 \mathrm{E}-05$ \\
\hline $10 / 4 / 2018$ & Station 22 & L134 & $2.20 \mathrm{E}-05$ & $7.281 \mathrm{E}-07$ & $1.566 \mathrm{E}-08$ & 1.207E-05 & $1.094 \mathrm{E}-09$ & $1.083 \mathrm{E}-06$ & $1.904 \mathrm{E}-10$ & 7.617E-10 & $6.236 \mathrm{E}-09$ & $8.093 \mathrm{E}-10$ & $1.471 \mathrm{E}-08$ & $9.521 \mathrm{E}-11$ & $2.856 \mathrm{E}-10$ & 1.40E-05 \\
\hline $11 / 12 / 2018$ & Station 22 & L134 & $2.00 \mathrm{E}-05$ & $1.004 \mathrm{E}-06$ & $1.885 \mathrm{E}-08$ & $1.296 \mathrm{E}-05$ & 1.637E-09 & $1.158 \mathrm{E}-06$ & $3.808 \mathrm{E}-10$ & $1.618 \mathrm{E}-09$ & 9.997E-09 & 7.617E-10 & 1.609E-08 & $9.521 \mathrm{E}-11$ & $3.332 \mathrm{E}-10$ & 1.30E-05 \\
\hline $12 / 12 / 2018$ & Station 22 & L134 & $2.30 \mathrm{E}-05$ & $6.918 \mathrm{E}-07$ & $1.549 \mathrm{E}-08$ & $1.132 \mathrm{E}-05$ & $1.171 \mathrm{E}-09$ & $8.846 \mathrm{E}-07$ & $3.808 \mathrm{E}-10$ & 1.190E-09 & $7.712 \mathrm{E}-09$ & $7.141 \mathrm{E}-10$ & $1.418 \mathrm{E}-08$ & 4.760E-11 & $2.856 \mathrm{E}-10$ & $1.20 \mathrm{E}-05$ \\
\hline $1 / 9 / 2019$ & Station 22 & L134 & $1.90 \mathrm{E}-05$ & $4.258 \mathrm{E}-07$ & $1.371 \mathrm{E}-08$ & $9.618 \mathrm{E}-06$ & $8.569 \mathrm{E}-10$ & $9.373 \mathrm{E}-07$ & $1.428 \mathrm{E}-10$ & $5.712 \mathrm{E}-10$ & $4.808 \mathrm{E}-09$ & 7.617E- 10 & $1.290 \mathrm{E}-08$ & $4.760 \mathrm{E}-11$ & $2.856 \mathrm{E}-10$ & $1.20 \mathrm{E}-05$ \\
\hline $4 / 25 / 2018$ & Station 3 & L1701 & $2.10 \mathrm{E}-05$ & $1.203 \mathrm{E}-06$ & 4.255E-08 & $1.192 \mathrm{E}-05$ & $3.884 \mathrm{E}-09$ & $1.015 \mathrm{E}-06$ & $6.665 \mathrm{E}-10$ & $3.713 \mathrm{E}-09$ & $1.980 \mathrm{E}-08$ & $6.188 \mathrm{E}-10$ & $1.014 \mathrm{E}-08$ & $2.380 \mathrm{E}-10$ & $6.665 \mathrm{E}-10$ & $1.30 \mathrm{E}-05$ \\
\hline $5 / 2 / 2018$ & Station 3 & L1701 & $2.30 \mathrm{E}-05$ & $5.132 \mathrm{E}-08$ & $3.275 \mathrm{E}-08$ & $1.203 \mathrm{E}-05$ & $2.699 \mathrm{E}-09$ & $1.121 \mathrm{E}-06$ & $5.236 \mathrm{E}-10$ & $3.284 \mathrm{E}-09$ & $1.728 \mathrm{E}-08$ & $9.521 \mathrm{E}-10$ & $1.009 \mathrm{E}-08$ & $4.428 \mathrm{E}-10$ & $6.665 \mathrm{E}-10$ & $1.20 \mathrm{E}-05$ \\
\hline $5 / 9 / 2018$ & Station 3 & L1701 & $1.90 \mathrm{E}-05$ & 1.439E-07 & 1.294E-08 & $9.199 \mathrm{E}-06$ & $1.428 \mathrm{E}-09$ & $9.141 \mathrm{E}-07$ & $1.428 \mathrm{E}-10$ & 4.760E-10 & 3.856E-09 & $3.808 \mathrm{E}-10$ & 7.617E-09 & $1.428 \mathrm{E}-10$ & $2.380 \mathrm{E}-10$ & 1.30E-05 \\
\hline $5 / 16 / 2018$ & Station 3 & L1701 & $1.70 \mathrm{E}-05$ & $1.171 \mathrm{E}-07$ & $1.188 \mathrm{E}-08$ & $1.019 \mathrm{E}-05$ & $8.569 \mathrm{E}-10$ & $9.219 \mathrm{E}-07$ & $9.521 \mathrm{E}-11$ & $1.904 \mathrm{E}-10$ & $2.475 \mathrm{E}-09$ & $3.808 \mathrm{E}-10$ & 7.664E-09 & $9.521 \mathrm{E}-11$ & $2.380 \mathrm{E}-10$ & $1.30 \mathrm{E}-05$ \\
\hline $5 / 22 / 2018$ & Station 3 & L1701 & $1.80 \mathrm{E}-05$ & $1.549 \mathrm{E}-07$ & $3.304 \mathrm{E}-08$ & $1.325 \mathrm{E}-05$ & 4.070E-09 & $1.330 \mathrm{E}-06$ & $7.141 \mathrm{E}-10$ & 4.475E-09 & $2.261 \mathrm{E}-08$ & $8.093 \mathrm{E}-10$ & 1.199E-08 & $1.428 \mathrm{E}-10$ & $7.141 \mathrm{E}-10$ & $1.30 \mathrm{E}-05$ \\
\hline $5 / 30 / 2018$ & Station 3 & L1701 & $2.40 \mathrm{E}-05$ & $1.754 \mathrm{E}-07$ & 2.929E-08 & $1.241 \mathrm{E}-05$ & $3.941 \mathrm{E}-09$ & $9.920 \mathrm{E}-07$ & $7.141 \mathrm{E}-10$ & 5.189E-09 & $2.666 \mathrm{E}-08$ & $7.141 \mathrm{E}-10$ & $1.252 \mathrm{E}-08$ & $1.428 \mathrm{E}-10$ & $6.665 \mathrm{E}-10$ & $1.30 \mathrm{E}-05$ \\
\hline $6 / 6 / 2018$ & Station 3 & L1701 & $2.30 \mathrm{E}-05$ & $1.431 \mathrm{E}-07$ & $1.392 \mathrm{E}-08$ & 1.120E-05 & $1.371 \mathrm{E}-09$ & $1.013 \mathrm{E}-06$ & $2.380 \mathrm{E}-10$ & $3.808 \mathrm{E}-10$ & 3.856E-09 & $4.284 \mathrm{E}-10$ & $1.085 \mathrm{E}-08$ & $1.904 \mathrm{E}-10$ & 3.332E-10 & $1.30 \mathrm{E}-05$ \\
\hline $7 / 11 / 2018$ & Station 3 & L1701 & $2.20 \mathrm{E}-05$ & 7.163E-07 & 9.335E-09 & $8.270 \mathrm{E}-06$ & $9.140 \mathrm{E}-10$ & 5.995E-07 & $1.904 \mathrm{E}-10$ & 7.617E-10 & 4.903E-09 & $3.332 \mathrm{E}-10$ & 8.093E-09 & 4.760E-11 & $2.380 \mathrm{E}-10$ & $1.30 \mathrm{E}-05$ \\
\hline $8 / 7 / 2018$ & Station 3 & L1701 & $2.70 \mathrm{E}-05$ & $7.059 \mathrm{E}-07$ & $2.494 \mathrm{E}-08$ & $1.215 \mathrm{E}-05$ & $3.713 \mathrm{E}-09$ & $8.769 \mathrm{E}-07$ & $6.188 \mathrm{E}-10$ & 4.332E-09 & $2.161 \mathrm{E}-08$ & $5.712 \mathrm{E}-10$ & $1.133 \mathrm{E}-08$ & $1.428 \mathrm{E}-10$ & $4.760 \mathrm{E}-10$ & $1.30 \mathrm{E}-05$ \\
\hline $9 / 6 / 2018$ & Station 3 & L1701 & 2.40E-05 & $5.027 \mathrm{E}-07$ & $9.197 \mathrm{E}-09$ & $7.606 \mathrm{E}-06$ & $7.141 \mathrm{E}-10$ & $7.958 \mathrm{E}-07$ & $1.428 \mathrm{E}-10$ & $1.904 \mathrm{E}-10$ & $1.904 \mathrm{E}-09$ & 3.332E-10 & 8.664E-09 & $9.521 \mathrm{E}-11$ & $1.904 \mathrm{E}-10$ & 1.40E-05 \\
\hline $10 / 4 / 2018$ & Station 3 & L1701 & $2.30 \mathrm{E}-05$ & $8.026 \mathrm{E}-07$ & $1.699 \mathrm{E}-08$ & 1.273E-05 & 1.313E-09 & $1.154 \mathrm{E}-06$ & $2.856 \mathrm{E}-10$ & $1.094 \mathrm{E}-09$ & 7.990E-09 & $7.141 \mathrm{E}-10$ & $1.542 \mathrm{E}-08$ & $9.521 \mathrm{E}-11$ & $3.808 \mathrm{E}-10$ & 1.40E-05 \\
\hline $11 / 12 / 2018$ & Station 3 & L1701 & $2.10 \mathrm{E}-05$ & $1.178 \mathrm{E}-06$ & $2.875 \mathrm{E}-08$ & 1.337E-05 & $3.889 \mathrm{E}-09$ & $1.213 \mathrm{E}-06$ & $6.188 \mathrm{E}-10$ & $4.808 \mathrm{E}-09$ & $2.470 \mathrm{E}-08$ & $8.569 \mathrm{E}-10$ & $1.566 \mathrm{E}-08$ & $1.428 \mathrm{E}-10$ & $6.665 \mathrm{E}-10$ & $1.30 \mathrm{E}-05$ \\
\hline $12 / 12 / 2018$ & Station 3 & L1701 & $1.80 \mathrm{E}-05$ & $8.857 \mathrm{E}-07$ & 2.907E-08 & $1.304 \mathrm{E}-05$ & 4.684E-09 & 1.007E-06 & $8.093 \mathrm{E}-10$ & $5.950 \mathrm{E}-09$ & $2.832 \mathrm{E}-08$ & $8.093 \mathrm{E}-10$ & $1.485 \mathrm{E}-08$ & $1.904 \mathrm{E}-10$ & 7.617E-10 & $1.30 \mathrm{E}-05$ \\
\hline
\end{tabular}




\begin{tabular}{|c|c|c|c|c|c|c|c|c|c|c|c|c|c|c|c|c|}
\hline Date & $\begin{array}{l}\text { Comment } \\
\mathrm{s}\end{array}$ & $\begin{array}{c}\text { MRM } \\
\text { Can } \\
\text { Sampled }\end{array}$ & $\begin{array}{l}\text { Total } \\
\text { press }\end{array}$ & Ch 1 & Ch 2 & Ch 3 & Ch 4 & Ch 5 & Ch 6 & Ch 7 & Ch 8 & Ch 9 & Ch 10 & Ch 11 & Ch 12 & $\begin{array}{c}\text { Ion } \\
\text { pressure }\end{array}$ \\
\hline $1 / 9 / 2019$ & Station 3 & L1701 & $1.80 \mathrm{E}-05$ & $5.568 \mathrm{E}-07$ & $2.013 \mathrm{E}-08$ & $1.142 \mathrm{E}-05$ & $2.570 \mathrm{E}-09$ & $1.118 \mathrm{E}-06$ & $4.760 \mathrm{E}-10$ & $1.713 \mathrm{E}-09$ & $1.009 \mathrm{E}-08$ & $8.569 \mathrm{E}-10$ & $1.528 \mathrm{E}-08$ & $1.428 \mathrm{E}-10$ & $4.760 \mathrm{E}-10$ & $1.20 \mathrm{E}-05$ \\
\hline $5 / 1 / 2019$ & Station 3 & L1702 & $4.10 \mathrm{E}-06$ & $7.451 \mathrm{E}-07$ & $3.684 \mathrm{E}-08$ & $2.754 \mathrm{E}-05$ & 4.189E-09 & $2.663 \mathrm{E}-06$ & $1.904 \mathrm{E}-10$ & $5.236 \mathrm{E}-10$ & 4.665E-09 & $6.665 \mathrm{E}-10$ & $1.294 \mathrm{E}-08$ & $4.760 \mathrm{E}-11$ & $2.856 \mathrm{E}-10$ & $3.50 \mathrm{E}-05$ \\
\hline $5 / 8 / 2019$ & Station 3 & L1702 & 3.40E-06 & $5.996 \mathrm{E}-07$ & 2.985E-08 & $2.759 \mathrm{E}-05$ & $3.142 \mathrm{E}-09$ & $2.829 \mathrm{E}-06$ & $2.380 \mathrm{E}-10$ & $5.236 \mathrm{E}-10$ & 4.094E-09 & $4.284 \mathrm{E}-10$ & $1.290 \mathrm{E}-08$ & $9.521 \mathrm{E}-11$ & $1.428 \mathrm{E}-10$ & $3.40 \mathrm{E}-05$ \\
\hline $5 / 15 / 2019$ & Station 3 & L1702 & $3.30 \mathrm{E}-06$ & $5.750 \mathrm{E}-07$ & $3.046 \mathrm{E}-08$ & 2.637E-05 & $3.999 \mathrm{E}-09$ & $2.818 \mathrm{E}-06$ & $9.521 \mathrm{E}-11$ & $3.808 \mathrm{E}-10$ & $6.618 \mathrm{E}-09$ & $5.236 \mathrm{E}-10$ & $1.218 \mathrm{E}-08$ & $9.521 \mathrm{E}-11$ & $1.428 \mathrm{E}-10$ & $3.30 \mathrm{E}-05$ \\
\hline $4 / 24 / 2019$ & Man A & $\begin{array}{l}\text { L1702, } \\
\text { L1704, } \\
\text { L1707, } \\
\text { L1708 }\end{array}$ & $4.50 \mathrm{E}-06$ & $6.708 \mathrm{E}-07$ & $5.698 \mathrm{E}-08$ & 2.667E-05 & $5.712 \mathrm{E}-09$ & $2.818 \mathrm{E}-06$ & $3.808 \mathrm{E}-10$ & $1.285 \mathrm{E}-09$ & $7.855 \mathrm{E}-09$ & $1.142 \mathrm{E}-09$ & $1.409 \mathrm{E}-08$ & $1.904 \mathrm{E}-10$ & $4.760 \mathrm{E}-10$ & $3.30 \mathrm{E}-05$ \\
\hline $5 / 1 / 2019$ & Station 4 & L1704 & 4.20E-06 & $6.089 \mathrm{E}-07$ & $3.193 \mathrm{E}-08$ & $2.706 \mathrm{E}-05$ & $2.618 \mathrm{E}-09$ & $2.660 \mathrm{E}-06$ & $2.380 \mathrm{E}-10$ & $5.712 \mathrm{E}-10$ & $4.808 \mathrm{E}-09$ & $5.712 \mathrm{E}-10$ & 1.309E-08 & $4.760 \mathrm{E}-11$ & $2.856 \mathrm{E}-10$ & $3.50 \mathrm{E}-05$ \\
\hline $5 / 8 / 2019$ & Station 4 & L1704 & $3.60 \mathrm{E}-06$ & $4.765 \mathrm{E}-07$ & $2.706 \mathrm{E}-08$ & $2.645 \mathrm{E}-05$ & $3.142 \mathrm{E}-09$ & $2.786 \mathrm{E}-06$ & $1.904 \mathrm{E}-10$ & 4.284E-10 & 4.094E-09 & $5.236 \mathrm{E}-10$ & $1.256 \mathrm{E}-08$ & $9.521 \mathrm{E}-11$ & $1.904 \mathrm{E}-10$ & $3.40 \mathrm{E}-05$ \\
\hline $5 / 15 / 2019$ & Station 4 & L1704 & 4.00E-06 & 4.907E-07 & 3.427E-08 & 2.417E-05 & $2.850 \mathrm{E}-09$ & $2.722 \mathrm{E}-06$ & $1.428 \mathrm{E}-10$ & $3.808 \mathrm{E}-10$ & $3.570 \mathrm{E}-09$ & $5.712 \mathrm{E}-10$ & $1.171 \mathrm{E}-08$ & $9.521 \mathrm{E}-11$ & $2.856 \mathrm{E}-10$ & $3.30 \mathrm{E}-05$ \\
\hline $5 / 1 / 2019$ & Station 7 & L1707 & 4.50E-06 & $6.659 \mathrm{E}-07$ & $2.825 \mathrm{E}-08$ & $2.560 \mathrm{E}-05$ & $2.094 \mathrm{E}-09$ & 2.557E-06 & $2.380 \mathrm{E}-10$ & $5.712 \mathrm{E}-10$ & 4.760E-09 & $4.760 \mathrm{E}-10$ & $1.275 \mathrm{E}-08$ & 4.760E-11 & $2.380 \mathrm{E}-10$ & $3.50 \mathrm{E}-05$ \\
\hline $5 / 8 / 2019$ & Station 7 & L1707 & 3.90E-06 & $5.315 \mathrm{E}-07$ & $2.985 \mathrm{E}-08$ & $2.624 \mathrm{E}-05$ & $2.618 \mathrm{E}-09$ & $2.697 \mathrm{E}-06$ & $1.904 \mathrm{E}-10$ & $4.760 \mathrm{E}-10$ & 4.141E-09 & $5.236 \mathrm{E}-10$ & $1.242 \mathrm{E}-08$ & $9.521 \mathrm{E}-11$ & $2.856 \mathrm{E}-10$ & $3.40 \mathrm{E}-05$ \\
\hline $5 / 15 / 2019$ & Station 7 & L1707 & $3.70 \mathrm{E}-06$ & $4.970 \mathrm{E}-07$ & $2.951 \mathrm{E}-08$ & $2.374 \mathrm{E}-05$ & $2.856 \mathrm{E}-09$ & $2.389 \mathrm{E}-06$ & $1.428 \mathrm{E}-10$ & $3.808 \mathrm{E}-10$ & $3.142 \mathrm{E}-09$ & $3.808 \mathrm{E}-10$ & $1.028 \mathrm{E}-08$ & $1.428 \mathrm{E}-10$ & $1.904 \mathrm{E}-10$ & $3.30 \mathrm{E}-05$ \\
\hline $5 / 1 / 2019$ & Station 8 & L1708 & $5.30 \mathrm{E}-06$ & $5.916 \mathrm{E}-07$ & $3.275 \mathrm{E}-08$ & $2.443 \mathrm{E}-05$ & $2.094 \mathrm{E}-09$ & $2.474 \mathrm{E}-06$ & $2.380 \mathrm{E}-10$ & $5.712 \mathrm{E}-10$ & $4.570 \mathrm{E}-09$ & $6.665 \mathrm{E}-10$ & $1.252 \mathrm{E}-08$ & $9.521 \mathrm{E}-11$ & $2.856 \mathrm{E}-10$ & $3.50 \mathrm{E}-05$ \\
\hline $5 / 8 / 2019$ & Station 8 & L1708 & 4.00E-06 & 4.686E-07 & $2.985 \mathrm{E}-08$ & $2.634 \mathrm{E}-05$ & $2.618 \mathrm{E}-09$ & $2.666 \mathrm{E}-06$ & $1.428 \mathrm{E}-10$ & $4.760 \mathrm{E}-10$ & $3.951 \mathrm{E}-09$ & $5.712 \mathrm{E}-10$ & $1.213 \mathrm{E}-08$ & $9.521 \mathrm{E}-11$ & $2.856 \mathrm{E}-10$ & $3.40 \mathrm{E}-05$ \\
\hline $5 / 15 / 2019$ & Station 8 & L1708 & $3.80 \mathrm{E}-06$ & 4.123E- 07 & $2.666 \mathrm{E}-08$ & $2.395 \mathrm{E}-05$ & $1.713 \mathrm{E}-09$ & $2.343 \mathrm{E}-06$ & $9.521 \mathrm{E}-11$ & $3.332 \mathrm{E}-10$ & $3.142 \mathrm{E}-09$ & $4.760 \mathrm{E}-10$ & 9.997E-09 & $4.760 \mathrm{E}-11$ & $1.428 \mathrm{E}-10$ & $3.30 \mathrm{E}-05$ \\
\hline
\end{tabular}




\section{Appendix D \\ MRM RGA Calibration Data}




\begin{tabular}{|c|c|c|c|c|c|c|c|c|c|c|c|c|}
\hline Date & Ch 1 & Ch 2 & Ch 3 & Ch 4 & Ch 5 & Ch 6 & Ch 7 & Ch 8 & Ch 9 & Ch 10 & Ch 11 & Ch 12 \\
\hline AMU & 4 & 28 & 40 & 44 & 20 & 16 & 17 & 18 & 32 & 36 & 12 & 14 \\
\hline $5 / 28 / 2014$ & 8.6 & 4.1 & 15 & 19 & 1.7 & & 1 & 1 & 1 & 1 & & \\
\hline $6 / 4 / 2014$ & 8.3 & 4.2 & 15 & 18 & 1.7 & & 1 & 1 & 1 & 1 & & \\
\hline $6 / 11 / 2014$ & 8.3 & 3.8 & 8 & 18 & 1.5 & & 1 & 1 & 1 & 1 & & \\
\hline $6 / 17 / 2014$ & 8.3 & 3.8 & 8.2 & 17 & 1.6 & & 1 & 1 & 1 & 1 & & \\
\hline $6 / 26 / 2014$ & 8.3 & 3.6 & 9.5 & 17 & 1.6 & & 1 & 1 & 1 & 1 & & \\
\hline $7 / 2 / 2014$ & 8.5 & 3.6 & 6.5 & 12 & 1.3 & & 1 & 1 & 1 & 1 & & \\
\hline $7 / 9 / 2014$ & 9 & 3.4 & 4.8 & 7.5 & 1.3 & & 1 & 1 & 1 & 1 & & \\
\hline $7 / 16 / 2014$ & 9 & 3.4 & 4.8 & 7.5 & 1.2 & & 1 & 1 & 1 & 1 & & \\
\hline $12 / 10 / 2014$ & 10 & 2.6 & 2.9 & 4.8 & 1.5 & & 1 & 1 & 1 & 1 & & \\
\hline $12 / 17 / 2014$ & 10 & 2.6 & 2.8 & 5.2 & 1.3 & & 1 & 1 & 1 & 1 & & \\
\hline $12 / 22 / 2014$ & 9.8 & 2.5 & 2.7 & 5.2 & 1.2 & & 1 & 1 & 1 & 1 & & \\
\hline $1 / 7 / 2015$ & 9.8 & 2.4 & 2.9 & 5.2 & 1.1 & & 1 & 1 & 1 & 1 & & \\
\hline $1 / 14 / 2015$ & 8.5 & 2.3 & 2.7 & 5.2 & 1.1 & & 1 & 1 & 1 & 1 & & \\
\hline $1 / 21 / 2015$ & 8.5 & 3.1 & 3.3 & 6 & 1.2 & & 1 & 1 & 1 & 1 & & \\
\hline $1 / 28 / 2015$ & 9.5 & 3.1 & 3.3 & 6.5 & 1.2 & & 1 & 1 & 1 & 1 & & \\
\hline $3 / 28 / 2017$ & 11 & 3.7 & 5.5 & 7.6 & 1.3 & & 1 & 1 & 1 & 1 & & \\
\hline $4 / 27 / 2017$ & 11 & 3 & 3.2 & 4.5 & 1.3 & & 1 & 1 & 1 & 1 & 1 & 3.7 \\
\hline $5 / 25 / 2017$ & 19 & 2.4 & 2.5 & 3.4 & 1.6 & & 1 & 1 & 1 & 1 & 1 & 1 \\
\hline $5 / 31 / 2017$ & 14 & 2.9 & 3.2 & 5.9 & 1.4 & & 1 & 1 & 1 & 1 & 1 & 1 \\
\hline $6 / 8 / 2017$ & 14 & 3.3 & 3.9 & 9 & 1.4 & 1 & 1 & 1 & 1 & 1 & 1 & 1 \\
\hline $6 / 14 / 2017$ & 14 & 4.5 & 9.3 & 14 & 16 & 1 & 1 & 1 & 1 & 1 & 1 & 1 \\
\hline $6 / 21 / 2017$ & 15 & 3.7 & 5.5 & 11 & 1.5 & 1 & 1 & 1 & 1 & 1 & 1 & 1 \\
\hline $6 / 28 / 2017$ & 14 & 4 & 4.5 & 11 & 1.5 & 1 & 1 & 1 & 1 & 1 & 1 & 1 \\
\hline $7 / 6 / 2017$ & 13 & 4.8 & 9 & 13 & 1.5 & 1 & 1 & 1 & 1 & 1 & 1 & 1 \\
\hline $7 / 12 / 2017$ & 14 & 3.7 & 6.5 & 12 & 1.5 & 1 & 1 & 1 & 1 & 1 & 1 & 1 \\
\hline $7 / 19 / 2017$ & 14 & 4.2 & 7.5 & 12 & 1.5 & 1 & 1 & 1 & 1 & 1 & 1 & 1 \\
\hline $7 / 25 / 2017$ & 14 & 4.5 & 7.5 & 12 & 1.5 & 1 & 1 & 1 & 1 & 1 & 1 & 1 \\
\hline $8 / 2 / 2017$ & 15 & 3.8 & 4.3 & 10 & 1.5 & 1 & 1 & 1 & 1 & 1 & 1 & 1 \\
\hline $8 / 9 / 2017$ & 15 & 4.1 & 4.7 & 12 & 1.6 & 1 & 1 & 1 & 1 & 1 & 1 & 1 \\
\hline $8 / 16 / 2017$ & 15 & 3.9 & 4.5 & 9.7 & 1.5 & 1 & 1 & 1 & 1 & 1 & 1 & 1 \\
\hline $8 / 24 / 2017$ & 15 & 3.9 & 4.5 & 9.7 & 1.5 & 1 & 1 & 1 & 1 & 1 & 1 & 1 \\
\hline $11 / 6 / 2017$ & 12 & 2.7 & 3.1 & 5.3 & 1.1 & 1 & 1 & 1 & 1 & 1 & 1 & 1 \\
\hline $12 / 6 / 2017$ & 13 & 3.1 & 3.5 & 6.5 & 1.2 & 1 & 1 & 1 & 1 & 1 & 1 & 1 \\
\hline $1 / 10 / 2018$ & 13 & 3.2 & 3.6 & 6.2 & 1.2 & 1 & 1 & 1 & 1 & 1 & 1 & 1 \\
\hline $2 / 7 / 2018$ & 13 & 2.9 & 3.2 & 6 & 1.2 & 1 & 1 & 1 & 1 & 1 & 1 & 1 \\
\hline $3 / 5 / 2018$ & 13 & 2.9 & 3.2 & 6 & 1.2 & 1 & 1 & 1 & 1 & 1 & 1 & 1 \\
\hline $4 / 4 / 2018$ & 18 & 3.9 & 4.2 & 6.5 & 1.5 & 1 & 1 & 1 & 1 & 1 & 1 & 1 \\
\hline $4 / 25 / 2018$ & 18 & 4.1 & 4.3 & 6.8 & 1.6 & 1 & 1 & 1 & 1 & 1 & 1 & 1 \\
\hline $5 / 2 / 2018$ & 22 & 4 & 4.5 & 6.3 & 1.9 & 1 & 1 & 1 & 1 & 1 & 1 & 1 \\
\hline $5 / 9 / 2018$ & 21 & 4 & 4.3 & 6 & 1.9 & 1 & 1 & 1 & 1 & 1 & 1 & 1 \\
\hline $5 / 16 / 2018$ & 20 & 3.9 & 4.3 & 6 & 1.9 & 1 & 1 & 1 & 1 & 1 & 1 & 1 \\
\hline
\end{tabular}




\begin{tabular}{|c|c|c|c|c|c|c|c|c|c|c|c|c|}
\hline Date & Ch 1 & Ch 2 & Ch 3 & Ch 4 & Ch 5 & Ch 6 & Ch 7 & Ch 8 & Ch 9 & Ch 10 & Ch 11 & Ch 12 \\
\hline $5 / 22 / 2018$ & 21 & 3.9 & 4.1 & 5.7 & 1.9 & 1 & 1 & 1 & 1 & 1 & 1 & 1 \\
\hline $5 / 30 / 2018$ & 19 & 3.4 & 3.7 & 4.6 & 1.5 & 1 & 1 & 1 & 1 & 1 & 1 & 1 \\
\hline $6 / 6 / 2018$ & 18 & 3.4 & 3.7 & 4.8 & 1.6 & 1 & 1 & 1 & 1 & 1 & 1 & 1 \\
\hline $7 / 11 / 2018$ & 22 & 3.7 & 3.8 & 4.8 & 1.7 & 1 & 1 & 1 & 1 & 1 & 1 & 1 \\
\hline $8 / 7 / 2018$ & 22 & 4 & 4.2 & 5.2 & 1.8 & 1 & 1 & 1 & 1 & 1 & 1 & 1 \\
\hline $9 / 6 / 2018$ & 24 & 4.2 & 3.8 & 5 & 2.4 & 1 & 1 & 1 & 1 & 1 & 1 & 1 \\
\hline $9 / 13 / 2018$ & 21 & 3.1 & 3.1 & 4.3 & 1.7 & 1 & 1 & 1 & 1 & 1 & 1 & 1 \\
\hline $9 / 20 / 2018$ & 21 & 3.4 & 3.4 & 4.5 & 1.9 & 1 & 1 & 1 & 1 & 1 & 1 & 1 \\
\hline $9 / 27 / 2018$ & 23 & 3.5 & 3.4 & 4.5 & 1.9 & 1 & 1 & 1 & 1 & 1 & 1 & 1 \\
\hline $10 / 4 / 2018$ & 23 & 3.5 & 3.4 & 4.6 & 1.9 & 1 & 1 & 1 & 1 & 1 & 1 & 1 \\
\hline $10 / 11 / 2018$ & 25 & 3.5 & 3.5 & 4.7 & 1.9 & 1 & 1 & 1 & 1 & 1 & 1 & 1 \\
\hline $11 / 12 / 2018$ & 22 & 3.3 & 3.3 & 4.3 & 1.9 & 1 & 1 & 1 & 1 & 1 & 1 & 1 \\
\hline $11 / 13 / 2018$ & 22 & 3.3 & 3.3 & 4.3 & 1.9 & 1 & 1 & 1 & 1 & 1 & 1 & 1 \\
\hline $12 / 12 / 2018$ & 21 & 3.1 & 3.2 & 4.1 & 1.6 & 1 & 1 & 1 & 1 & 1 & 1 & 1 \\
\hline $1 / 9 / 2019$ & 16 & 3 & 2.9 & 3.6 & 1.9 & 1 & 1 & 1 & 1 & 1 & 1 & 1 \\
\hline $4 / 24 / 2019$ & 52 & 9 & 8 & 10 & 5.2 & 1 & 1 & 1 & 1 & 1 & 1 & 1 \\
\hline $5 / 1 / 2019$ & 52 & 8.6 & 8 & 11 & 5.2 & 1 & 1 & 1 & 1 & 1 & 1 & 1 \\
\hline $5 / 8 / 2019$ & 55 & 9.8 & 8.3 & 11 & 5.7 & 1 & 1 & 1 & 1 & 1 & 1 & 1 \\
\hline $5 / 15 / 2019$ & 61 & 10 & 8.5 & 12 & 6 & 1 & 1 & 1 & 1 & 1 & 1 & 1 \\
\hline
\end{tabular}




\section{Appendix D \\ MRM Can Temperature Data}




\begin{tabular}{|c|c|c|c|c|c|c|c|c|c|c|c|c|c|}
\hline Date & $\begin{array}{c}\text { Station } \\
3\end{array}$ & $\begin{array}{c}\text { Station } \\
4\end{array}$ & $\begin{array}{c}\text { Station } \\
11\end{array}$ & $\begin{array}{c}\text { Station } \\
12\end{array}$ & $\begin{array}{c}\text { Station } \\
13\end{array}$ & $\begin{array}{c}\text { Station } \\
15\end{array}$ & $\begin{array}{c}\text { Station } \\
21\end{array}$ & $\begin{array}{c}\text { Station } \\
22\end{array}$ & $\begin{array}{c}\text { Station } \\
25\end{array}$ & $\begin{array}{c}\text { Station } \\
26\end{array}$ & $\begin{array}{c}\text { Station } \\
27\end{array}$ & $\begin{array}{c}\text { Station } \\
7\end{array}$ & $\begin{array}{c}\text { Station } \\
8\end{array}$ \\
\hline $6 / 17 / 2014$ & 288 & 298 & & & & & & & & & & & \\
\hline $6 / 17 / 2014$ & 288 & 298 & & & & & & & & & & & \\
\hline $6 / 26 / 2014$ & 289 & 297 & & & & & & & & & & & \\
\hline $6 / 26 / 2014$ & 289 & 297 & & & & & & & & & & & \\
\hline $7 / 2 / 2014$ & 289 & 297 & & & & & & & & & & & \\
\hline $7 / 2 / 2014$ & 289 & 297 & & & & & & & & & & & \\
\hline $7 / 9 / 2014$ & 291 & 296 & & & & & & & & & & & \\
\hline $7 / 9 / 2014$ & 291 & 296 & & & & & & & & & & & \\
\hline $7 / 16 / 2014$ & 295 & 295 & & & & & & & & & & & \\
\hline $7 / 16 / 2014$ & 295 & 295 & & & & & & & & & & & \\
\hline $3 / 28 / 2017$ & No Data & $\begin{array}{c}\text { No } \\
\text { Data }\end{array}$ & $\begin{array}{l}\text { No } \\
\text { Data }\end{array}$ & $\begin{array}{l}\text { No } \\
\text { Data }\end{array}$ & & & $\begin{array}{l}\text { No } \\
\text { Data }\end{array}$ & $\begin{array}{c}\text { No } \\
\text { Data }\end{array}$ & $\begin{array}{c}\text { No } \\
\text { Data }\end{array}$ & $\begin{array}{l}\text { No } \\
\text { Data }\end{array}$ & & & \\
\hline $4 / 27 / 2017$ & & & & & & & 189 & 212 & 160 & 173 & & & \\
\hline $5 / 25 / 2017$ & 288 & & & & & & 193 & 212 & 165 & 175 & & & \\
\hline $6 / 21 / 2017$ & 286 & & & & & & 195 & 212 & 166 & 175 & & & \\
\hline $7 / 25 / 2017$ & 286 & & & & & & 196 & 212 & 166 & 174 & & & \\
\hline $8 / 24 / 2017$ & 288 & & & & & & 257 & 321 & 263 & 314 & & & \\
\hline $11 / 6 / 2017$ & 286 & & & & & & 254 & 316 & 261 & 316 & & & \\
\hline $1 / 10 / 2018$ & 289 & & & & & & 265 & 314 & 260 & 317 & & & \\
\hline $3 / 5 / 2018$ & 291 & & & & & & 263 & 313 & 258 & 313 & & & \\
\hline $4 / 4 / 2018$ & & & & & & & 259 & 313 & 269 & 314 & 288 & & \\
\hline $5 / 2 / 2018$ & 299 & & & & & & 262 & 313 & 270 & 314 & 288 & & \\
\hline $6 / 6 / 2018$ & 302 & & & & & & 267 & 312 & 270 & 315 & 288 & & \\
\hline $7 / 11 / 2018$ & 300 & & & & & & 262 & 312 & 270 & 316 & 287 & & \\
\hline $8 / 7 / 2018$ & 305 & & & & & & 257 & 311 & 270 & 314 & 287 & & \\
\hline $9 / 6 / 2018$ & 305 & & 300 & & 297 & 317 & 265 & 306 & 267 & 313 & 281 & & \\
\hline $10 / 4 / 2018$ & 306 & & 300 & & 295 & 317 & 262 & 306 & 266 & 313 & 281 & & \\
\hline $11 / 12 / 2018$ & 307 & & 296 & & 296 & 315 & 257 & 300 & 264 & 312 & 279 & & \\
\hline $12 / 12 / 2018$ & 308 & & 296 & & 296 & 317 & 251 & 301 & 265 & 313 & 279 & & \\
\hline $1 / 9 / 2019$ & 322 & & 301 & & 305 & 323 & 256 & 307 & 263 & 312 & 287 & & \\
\hline $5 / 28 / 2014$ & 283 & 288 & & & & & & & & & & & \\
\hline $5 / 28 / 2014$ & 283 & 288 & & & & & & & & & & & \\
\hline $6 / 4 / 2014$ & 290 & 296 & & & & & & & & & & & \\
\hline $6 / 4 / 2014$ & 290 & 296 & & & & & & & & & & & \\
\hline $6 / 11 / 2014$ & 287 & 295 & & & & & & & & & & & \\
\hline $6 / 11 / 2014$ & 287 & 295 & & & & & & & & & & & \\
\hline
\end{tabular}




\begin{tabular}{|c|c|c|c|c|c|c|c|c|c|c|c|c|c|}
\hline Date & $\begin{array}{c}\text { Station } \\
3 \\
\end{array}$ & $\begin{array}{c}\text { Station } \\
4 \\
\end{array}$ & $\begin{array}{c}\text { Station } \\
11 \\
\end{array}$ & $\begin{array}{c}\text { Station } \\
12 \\
\end{array}$ & $\begin{array}{c}\text { Station } \\
13 \\
\end{array}$ & $\begin{array}{c}\text { Station } \\
15 \\
\end{array}$ & $\begin{array}{c}\text { Station } \\
21 \\
\end{array}$ & $\begin{array}{c}\text { Station } \\
22 \\
\end{array}$ & $\begin{array}{c}\text { Station } \\
25 \\
\end{array}$ & $\begin{array}{c}\text { Station } \\
26 \\
\end{array}$ & $\begin{array}{c}\text { Station } \\
27 \\
\end{array}$ & $\begin{array}{c}\text { Station } \\
7 \\
\end{array}$ & $\begin{array}{c}\text { Station } \\
8 \\
\end{array}$ \\
\hline $9 / 20 / 2018$ & 304 & & 301 & & 296 & 317 & 263 & 307 & 267 & 313 & 281 & & \\
\hline $9 / 27 / 2018$ & 304 & & 298 & & 294 & 316 & 263 & 308 & 266 & 313 & 281 & & \\
\hline $10 / 4 / 2018$ & 304 & & 296 & & 395 & 316 & 263 & 306 & 266 & 313 & 280 & & \\
\hline $10 / 11 / 2018$ & 307 & & 296 & & 296 & 316 & 260 & 304 & 266 & 313 & 280 & & \\
\hline $11 / 13 / 2018$ & 307 & & 296 & & 295 & 315 & 258 & 302 & 264 & 312 & 279 & & \\
\hline $12 / 12 / 2018$ & 308 & & 291 & & 296 & 317 & 258 & 303 & 265 & 313 & 280 & & \\
\hline $1 / 9 / 2019$ & 323 & & 297 & & 306 & 323 & 263 & 306 & 264 & 312 & 287 & & \\
\hline $9 / 5 / 2018$ & 304 & & 296 & & 294 & 315 & 265 & 306 & 267 & 314 & 280 & & \\
\hline $9 / 13 / 2018$ & 305 & & 303 & & 298 & 318 & 264 & 308 & 267 & 314 & 281 & & \\
\hline $5 / 31 / 2017$ & 285 & & & & & & 192 & 211 & 163 & 174 & & & \\
\hline $5 / 31 / 2017$ & 285 & & & & & & 192 & 211 & 163 & 174 & & & \\
\hline $6 / 8 / 2017$ & 290 & & & & & & 193 & 212 & 164 & 174 & & & \\
\hline $6 / 8 / 2017$ & 290 & & & & & & 193 & 212 & 164 & 174 & & & \\
\hline $6 / 8 / 2017$ & 290 & & & & & & 193 & 212 & 164 & 174 & & & \\
\hline $6 / 14 / 2017$ & 288 & & & & & & 192 & 210 & 162 & 174 & & & \\
\hline $6 / 14 / 2017$ & 288 & & & & & & 192 & 210 & 162 & 174 & & & \\
\hline $6 / 21 / 2017$ & 286 & & & & & & 195 & 212 & 166 & 175 & & & \\
\hline $6 / 28 / 2017$ & 285 & & & & & & 196 & 210 & 164 & 173 & & & \\
\hline $6 / 28 / 2017$ & 285 & & & & & & 196 & 210 & 164 & 173 & & & \\
\hline $7 / 6 / 2017$ & 285 & & & & & & 195 & 211 & 164 & 173 & & & \\
\hline $7 / 12 / 2017$ & 285 & & & & & & 196 & 212 & 165 & 174 & & & \\
\hline $7 / 19 / 2017$ & 287 & & & & & & 195 & 211 & 166 & 174 & & & \\
\hline $7 / 19 / 2017$ & 287 & & & & & & 195 & 211 & 166 & 174 & & & \\
\hline $7 / 25 / 2017$ & 286 & & & & & & 196 & 212 & 166 & 174 & & & \\
\hline $8 / 2 / 2017$ & 286 & & & & & & 196 & 211 & 164 & 173 & & & \\
\hline $8 / 9 / 2017$ & 285 & & & & & & 196 & 211 & 164 & 173 & & & \\
\hline $8 / 16 / 2017$ & 286 & & & & & & 196 & 210 & 164 & 173 & & & \\
\hline $8 / 16 / 2017$ & 286 & & & & & & 196 & 210 & 164 & 173 & & & \\
\hline $8 / 24 / 2017$ & 286 & & & & & & 196 & 210 & 164 & 173 & & & \\
\hline $11 / 6 / 2017$ & 285 & & & & & & 264 & 317 & 261 & 316 & & & \\
\hline $12 / 6 / 2017$ & 286 & & & & & & 262 & 313 & 258 & 314 & & & \\
\hline $1 / 10 / 2018$ & 289 & & & & & & 265 & 314 & 260 & 317 & & & \\
\hline $2 / 7 / 2018$ & 291 & & & & & & 264 & 313 & 259 & 313 & & & \\
\hline $3 / 5 / 2018$ & $291 / 219$ & & & & & & 263 & 314 & 258 & 313 & & & \\
\hline $4 / 4 / 2018$ & & & & & & & 264 & 312 & 268 & 314 & 288 & & \\
\hline $5 / 2 / 2018$ & 299 & & & & & & 267 & 314 & 270 & 314 & 289 & & \\
\hline
\end{tabular}




\begin{tabular}{|c|c|c|c|c|c|c|c|c|c|c|c|c|c|}
\hline Date & $\begin{array}{c}\text { Station } \\
3\end{array}$ & $\begin{array}{c}\text { Station } \\
4\end{array}$ & $\begin{array}{c}\text { Station } \\
11\end{array}$ & $\begin{array}{c}\text { Station } \\
12\end{array}$ & $\begin{array}{c}\text { Station } \\
13\end{array}$ & $\begin{array}{c}\text { Station } \\
15\end{array}$ & $\begin{array}{c}\text { Station } \\
21\end{array}$ & $\begin{array}{c}\text { Station } \\
22\end{array}$ & $\begin{array}{c}\text { Station } \\
25\end{array}$ & $\begin{array}{c}\text { Station } \\
26\end{array}$ & $\begin{array}{c}\text { Station } \\
27\end{array}$ & $\begin{array}{c}\text { Station } \\
7\end{array}$ & $\begin{array}{c}\text { Station } \\
8\end{array}$ \\
\hline $6 / 6 / 2018$ & 300 & & & & & & 267 & 313 & 270 & 309 & 288 & & \\
\hline $7 / 11 / 2018$ & 302 & & & & & & 267 & 312 & 270 & 316 & 288 & & \\
\hline $8 / 7 / 2018$ & 305 & & & & & & 266 & 310 & 270 & 315 & 287 & & \\
\hline $9 / 6 / 2018$ & 304 & & 299 & & 296 & 316 & 266 & 309 & 267 & 309 & 281 & & \\
\hline $10 / 4 / 2018$ & 306 & & 300 & & 297 & 317 & 264 & 308 & 266 & 307 & 281 & & \\
\hline $11 / 12 / 2018$ & 307 & & 296 & & 295 & 315 & 258 & 302 & 264 & 306 & 279 & & \\
\hline $12 / 12 / 2018$ & 308 & & 296 & & 296 & 316 & 257 & 303 & 264 & 313 & 279 & & \\
\hline $1 / 9 / 2019$ & 322 & & 301 & & 306 & 323 & 262 & 306 & 264 & 312 & 287 & & \\
\hline $9 / 20 / 2018$ & 305 & & 301 & & 292 & 317 & 264 & 307 & 267 & 313 & 281 & & \\
\hline $9 / 27 / 2018$ & 304 & & 298 & & 294 & 316 & 263 & 308 & 266 & 313 & 281 & & \\
\hline $10 / 4 / 2018$ & 304 & & 300 & & 292 & 316 & 262 & 307 & 266 & 313 & 280 & & \\
\hline $10 / 11 / 2018$ & 307 & & 293 & & 296 & 315 & 260 & 304 & 266 & 313 & 280 & & \\
\hline $11 / 13 / 2018$ & 307 & & 291 & & 295 & 315 & 257 & 302 & 263 & 312 & 279 & & \\
\hline $12 / 12 / 2018$ & 309 & & 296 & & 290 & 317 & 259 & 302 & 265 & 313 & 279 & & \\
\hline $1 / 9 / 2019$ & 322 & & 302 & & 303 & 324 & 262 & 306 & 264 & 312 & 287 & & \\
\hline $12 / 22 / 2014$ & 191 & 223 & 305 & 308 & & & & & & & & & \\
\hline $12 / 22 / 2014$ & 191 & 223 & 305 & 308 & & & & & & & & & \\
\hline $1 / 7 / 2015$ & 192 & 226 & 295 & 307 & & & & & & & & & \\
\hline $1 / 7 / 2015$ & 192 & 226 & 295 & 307 & & & & & & & & & \\
\hline $1 / 14 / 2015$ & 194 & 221 & 304 & 306 & & & & & & & & & \\
\hline $1 / 21 / 2015$ & 185 & 227 & 298 & 309 & & & & & & & & & \\
\hline $1 / 28 / 2015$ & 191 & 221 & 304 & 305 & & & & & & & & & \\
\hline $3 / 28 / 2017$ & No Data & $\begin{array}{l}\text { No } \\
\text { Data }\end{array}$ & $\begin{array}{l}\text { No } \\
\text { Data }\end{array}$ & $\begin{array}{l}\text { No } \\
\text { Data }\end{array}$ & & & $\begin{array}{l}\text { No } \\
\text { Data }\end{array}$ & $\begin{array}{c}\text { No } \\
\text { Data }\end{array}$ & $\begin{array}{l}\text { No } \\
\text { Data }\end{array}$ & $\begin{array}{c}\text { No } \\
\text { Data }\end{array}$ & & & \\
\hline $4 / 27 / 2017$ & & & & & & & 189 & 212 & 160 & 173 & & & \\
\hline $5 / 25 / 2017$ & 288 & & & & & & 193 & 212 & 165 & 175 & & & \\
\hline $6 / 21 / 2017$ & 286 & & & & & & 195 & 212 & 166 & 175 & & & \\
\hline $7 / 25 / 2017$ & 286 & & & & & & 196 & 212 & 166 & 174 & & & \\
\hline $8 / 24 / 2017$ & 286 & & & & & & 266 & 322 & 256 & 317 & & & \\
\hline $11 / 6 / 2017$ & 286 & & & & & & 265 & 314 & 252 & 316 & & & \\
\hline $1 / 10 / 2018$ & 289 & & & & & & 265 & 314 & 260 & 317 & & & \\
\hline $3 / 5 / 2018$ & 291 & & & & & & 263 & 313 & 258 & 313 & & & \\
\hline $4 / 4 / 2018$ & & & & & & & 268 & 315 & 262 & 314 & 288 & & \\
\hline $5 / 2 / 2018$ & 299 & & & & & & 269 & 315 & 264 & 314 & 289 & & \\
\hline $6 / 6 / 2018$ & 300 & & & & & & 268 & 313 & 270 & 316 & 288 & & \\
\hline $7 / 11 / 2018$ & 300 & & & & & & 267 & 313 & 271 & 316 & 287 & & \\
\hline
\end{tabular}




\begin{tabular}{|c|c|c|c|c|c|c|c|c|c|c|c|c|c|}
\hline Date & $\begin{array}{c}\text { Station } \\
3\end{array}$ & $\begin{array}{c}\text { Station } \\
4\end{array}$ & $\begin{array}{c}\text { Station } \\
11\end{array}$ & $\begin{array}{c}\text { Station } \\
12\end{array}$ & $\begin{array}{c}\text { Station } \\
13\end{array}$ & $\begin{array}{c}\text { Station } \\
15\end{array}$ & $\begin{array}{c}\text { Station } \\
21\end{array}$ & $\begin{array}{c}\text { Station } \\
22\end{array}$ & $\begin{array}{c}\text { Station } \\
25\end{array}$ & $\begin{array}{c}\text { Station } \\
26\end{array}$ & $\begin{array}{c}\text { Station } \\
27\end{array}$ & $\begin{array}{c}\text { Station } \\
7\end{array}$ & $\begin{array}{c}\text { Station } \\
8\end{array}$ \\
\hline $8 / 7 / 2018$ & 304 & & & & & & 267 & 313 & 264 & 314 & 287 & & \\
\hline $9 / 6 / 2018$ & 305 & & 301 & & 297 & 317 & 267 & 303 & 267 & 314 & 281 & & \\
\hline $10 / 4 / 2018$ & 306 & & 300 & & 297 & 317 & 264 & 299 & 266 & 313 & 281 & & \\
\hline $11 / 12 / 2018$ & 307 & & 296 & & 295 & 315 & 259 & 295 & 264 & 313 & 279 & & \\
\hline $12 / 12 / 2018$ & 308 & & 296 & & 296 & 316 & 260 & 303 & 259 & 313 & 279 & & \\
\hline $1 / 9 / 2019$ & 322 & & 301 & & 306 & 323 & 262 & 306 & 257 & 312 & 287 & & \\
\hline $12 / 10 / 2014$ & 191 & 224 & 302 & 303 & & & & & & & & & \\
\hline $12 / 10 / 2014$ & 191 & 224 & 302 & 303 & & & & & & & & & \\
\hline $12 / 17 / 2014$ & 193 & 224 & 306 & 308 & & & & & & & & & \\
\hline $12 / 17 / 2014$ & 193 & 224 & 306 & 308 & & & & & & & & & \\
\hline $12 / 22 / 2014$ & 191 & 223 & 305 & 308 & & & & & & & & & \\
\hline $1 / 7 / 2015$ & 192 & 226 & 295 & 307 & & & & & & & & & \\
\hline $1 / 14 / 2015$ & 194 & 221 & 304 & 306 & & & & & & & & & \\
\hline $1 / 21 / 2015$ & 185 & 227 & 298 & 309 & & & & & & & & & \\
\hline $1 / 28 / 2015$ & 191 & 221 & 304 & 305 & & & & & & & & & \\
\hline $3 / 28 / 2017$ & No Data & $\begin{array}{l}\text { No } \\
\text { Data }\end{array}$ & $\begin{array}{c}\text { No } \\
\text { Data }\end{array}$ & $\begin{array}{c}\text { No } \\
\text { Data }\end{array}$ & & & $\begin{array}{l}\text { No } \\
\text { Data }\end{array}$ & $\begin{array}{l}\text { No } \\
\text { Data }\end{array}$ & $\begin{array}{l}\text { No } \\
\text { Data }\end{array}$ & $\begin{array}{l}\text { No } \\
\text { Data }\end{array}$ & & & \\
\hline $4 / 27 / 2017$ & & & & & & & 189 & 212 & 160 & 173 & & & \\
\hline $5 / 25 / 2017$ & 288 & & & & & & 193 & 212 & 165 & 175 & & & \\
\hline $6 / 21 / 2017$ & 286 & & & & & & 195 & 212 & 166 & 175 & & & \\
\hline $7 / 25 / 2017$ & 286 & & & & & & 196 & 212 & 166 & 174 & & & \\
\hline $8 / 24 / 2017$ & 286 & & & & & & 267 & 322 & 264 & 317 & & & \\
\hline $11 / 6 / 2017$ & 286 & & & & & & 265 & 315 & 260 & 311 & & & \\
\hline $1 / 10 / 2018$ & 289 & & & & & & 265 & 314 & 260 & 317 & & & \\
\hline $3 / 5 / 2018$ & 291 & & & & & & 263 & 313 & 258 & 313 & & & \\
\hline $4 / 4 / 2018$ & & & & & & & 267 & 315 & 269 & 306 & 287 & & \\
\hline $5 / 2 / 2018$ & 299 & & & & & & 268 & 315 & 271 & 308 & 289 & & \\
\hline $6 / 6 / 2018$ & 300 & & & & & & 268 & 313 & 264 & 315 & 288 & & \\
\hline $7 / 11 / 2018$ & 300 & & & & & & 268 & 313 & 271 & 309 & 287 & & \\
\hline $8 / 7 / 2018$ & 304 & & & & & & 267 & 313 & 271 & 307 & 286 & & \\
\hline $9 / 6 / 2018$ & 304 & & 299 & & 296 & 317 & 266 & 309 & 268 & 314 & 282 & & \\
\hline $10 / 4 / 2018$ & 306 & & 300 & & 297 & 317 & 264 & 306 & 260 & 313 & 281 & & \\
\hline $11 / 12 / 2018$ & 307 & & 296 & & 295 & 315 & 259 & 302 & 258 & 313 & 279 & & \\
\hline $12 / 12 / 2018$ & 308 & & 296 & & 296 & 317 & 258 & 303 & 264 & 306 & 279 & & \\
\hline $1 / 9 / 2019$ & 322 & & 301 & & 306 & 323 & 262 & 306 & 264 & 305 & 287 & & \\
\hline $9 / 20 / 2018$ & 305 & & 300 & & 296 & 317 & 264 & 308 & 267 & 314 & 281 & & \\
\hline
\end{tabular}




\begin{tabular}{|c|c|c|c|c|c|c|c|c|c|c|c|c|c|}
\hline Date & $\begin{array}{c}\text { Station } \\
3 \\
\end{array}$ & $\begin{array}{c}\text { Station } \\
4 \\
\end{array}$ & $\begin{array}{c}\text { Station } \\
11 \\
\end{array}$ & $\begin{array}{c}\text { Station } \\
12 \\
\end{array}$ & $\begin{array}{c}\text { Station } \\
13 \\
\end{array}$ & $\begin{array}{c}\text { Station } \\
15 \\
\end{array}$ & $\begin{array}{c}\text { Station } \\
21 \\
\end{array}$ & $\begin{array}{c}\text { Station } \\
22 \\
\end{array}$ & $\begin{array}{c}\text { Station } \\
25 \\
\end{array}$ & $\begin{array}{c}\text { Station } \\
26 \\
\end{array}$ & $\begin{array}{c}\text { Station } \\
27 \\
\end{array}$ & $\begin{array}{c}\text { Station } \\
7 \\
\end{array}$ & $\begin{array}{c}\text { Station } \\
8 \\
\end{array}$ \\
\hline $9 / 27 / 2018$ & 304 & & 298 & & 294 & 316 & 263 & 308 & 266 & 313 & 281 & & \\
\hline $10 / 4 / 2018$ & 304 & & 300 & & 297 & 316 & 262 & 307 & 266 & 313 & 280 & & \\
\hline $10 / 11 / 2018$ & 307 & & 299 & & 292 & 315 & 260 & 304 & 266 & 313 & 280 & & \\
\hline $11 / 13 / 2018$ & 307 & & 295 & & 290 & 316 & 257 & 302 & 263 & 312 & 279 & & \\
\hline $12 / 12 / 2018$ & 308 & & 297 & & 295 & 315 & 259 & 303 & 265 & 313 & 279 & & \\
\hline $1 / 9 / 2019$ & 322 & & 302 & & 307 & 323 & 261 & 306 & 264 & 312 & 287 & & \\
\hline $6 / 17 / 2014$ & 288 & 298 & & & & & & & & & & & \\
\hline $6 / 17 / 2014$ & 288 & 298 & & & & & & & & & & & \\
\hline $6 / 26 / 2014$ & 289 & 297 & & & & & & & & & & & \\
\hline $6 / 26 / 2014$ & 289 & 297 & & & & & & & & & & & \\
\hline $7 / 2 / 2014$ & 289 & 297 & & & & & & & & & & & \\
\hline $7 / 2 / 2014$ & 289 & 297 & & & & & & & & & & & \\
\hline $7 / 9 / 2014$ & 291 & 296 & & & & & & & & & & & \\
\hline $7 / 9 / 2014$ & 291 & 296 & & & & & & & & & & & \\
\hline $7 / 16 / 2014$ & 295 & 295 & & & & & & & & & & & \\
\hline $7 / 16 / 2014$ & 295 & 295 & & & & & & & & & & & \\
\hline $4 / 27 / 2017$ & & & & & & & 189 & 212 & 160 & 173 & & & \\
\hline $5 / 25 / 2017$ & 288 & & & & & & 193 & 212 & 165 & 175 & & & \\
\hline $6 / 21 / 2017$ & 286 & & & & & & 195 & 212 & 166 & 175 & & & \\
\hline $7 / 25 / 2017$ & 286 & & & & & & 196 & 212 & 166 & 174 & & & \\
\hline $8 / 24 / 2017$ & 288 & & & & & & 268 & 315 & 264 & 316 & & & \\
\hline $11 / 6 / 2017$ & 286 & & & & & & 255 & 315 & 261 & 316 & & & \\
\hline $1 / 10 / 2018$ & 289 & & & & & & 265 & 314 & 260 & 317 & & & \\
\hline $3 / 5 / 2018$ & 291 & & & & & & 263 & 313 & 258 & 313 & & & \\
\hline $4 / 4 / 2018$ & & & & & & & 269 & 308 & 269 & 314 & 288 & & \\
\hline $5 / 2 / 2018$ & 299 & & & & & & 269 & 308 & 270 & 314 & 289 & & \\
\hline $6 / 6 / 2018$ & 301 & & & & & & 260 & 312 & 270 & 316 & 288 & & \\
\hline $7 / 11 / 2018$ & 300 & & & & & & 268 & 305 & 268 & 316 & 287 & & \\
\hline $8 / 7 / 2018$ & 305 & & & & & & 268 & 304 & 269 & 314 & 287 & & \\
\hline $9 / 6 / 2018$ & 305 & & 299 & & 295 & 316 & 261 & 306 & 267 & 314 & 281 & & \\
\hline $10 / 4 / 2018$ & 306 & & 300 & & 296 & 317 & 258 & 305 & 266 & 313 & 281 & & \\
\hline $11 / 12 / 2018$ & 307 & & 296 & & 295 & 315 & 251 & 301 & 264 & 312 & 279 & & \\
\hline $12 / 12 / 2018$ & 308 & & 296 & & 295 & 317 & 259 & 294 & 264 & 312 & 279 & & \\
\hline $1 / 9 / 2019$ & 322 & & 301 & & 306 & 323 & 264 & 300 & 264 & 312 & 287 & & \\
\hline $4 / 25 / 2018$ & 292 & & & & & & 268 & 314 & 269 & 313 & 288 & & \\
\hline $5 / 2 / 2018$ & 298 & & & & & & 266 & 314 & 270 & 315 & 288 & & \\
\hline
\end{tabular}




\begin{tabular}{|c|c|c|c|c|c|c|c|c|c|c|c|c|c|}
\hline Date & $\begin{array}{c}\text { Station } \\
3\end{array}$ & $\begin{array}{c}\text { Station } \\
4\end{array}$ & $\begin{array}{c}\text { Station } \\
11\end{array}$ & $\begin{array}{c}\text { Station } \\
12\end{array}$ & $\begin{array}{c}\text { Station } \\
13\end{array}$ & $\begin{array}{c}\text { Station } \\
15\end{array}$ & $\begin{array}{c}\text { Station } \\
21\end{array}$ & $\begin{array}{c}\text { Station } \\
22\end{array}$ & $\begin{array}{c}\text { Station } \\
25\end{array}$ & $\begin{array}{c}\text { Station } \\
26\end{array}$ & $\begin{array}{c}\text { Station } \\
27\end{array}$ & $\begin{array}{c}\text { Station } \\
7\end{array}$ & $\begin{array}{c}\text { Station } \\
8\end{array}$ \\
\hline $5 / 9 / 2018$ & 297 & & & & & & 270 & 314 & 271 & 314 & 289 & & \\
\hline $5 / 16 / 2018$ & 298 & & & & & & 268 & 313 & 270 & 314 & 288 & & \\
\hline $5 / 22 / 2018$ & 297 & & & & & & 268 & 312 & 270 & 313 & 287 & & \\
\hline $5 / 30 / 2018$ & 297 & & & & & & 267 & 312 & 270 & 314 & 287 & & \\
\hline $6 / 6 / 2018$ & 302 & & & & & & 268 & 313 & 270 & 315 & 288 & & \\
\hline $7 / 11 / 2018$ & 304 & & & & & & 267 & 311 & 270 & 317 & 287 & & \\
\hline $8 / 7 / 2018$ & 305 & & & & & & 266 & 311 & 269 & 315 & 286 & & \\
\hline $9 / 6 / 2018$ & 303 & & 300 & & 297 & 317 & 265 & 307 & 268 & 313 & 282 & & \\
\hline $10 / 4 / 2018$ & 305 & & 300 & & 297 & 317 & 262 & 306 & 266 & 313 & 281 & & \\
\hline $11 / 12 / 2018$ & 307 & & 295 & & 294 & 315 & 258 & 301 & 263 & 312 & 279 & & \\
\hline $12 / 12 / 2018$ & 307 & & 295 & & 295 & 317 & 260 & 303 & 265 & 313 & 279 & & \\
\hline $1 / 9 / 2019$ & 321 & & 302 & & 307 & 325 & 262 & 307 & 264 & 312 & 288 & & \\
\hline $5 / 1 / 2019$ & 304 & 308 & & & & & & & & & & 313 & 303 \\
\hline $5 / 8 / 2019$ & 304 & 308 & & & & & & & & & & 314 & 303 \\
\hline $5 / 15 / 2019$ & 304 & 308 & & & & & & & & & & 315 & 302 \\
\hline $4 / 24 / 2019$ & 304 & 309 & & & & & & & & & & 315 & 303 \\
\hline $5 / 1 / 2019$ & 304 & 308 & & & & & & & & & & 313 & 303 \\
\hline $5 / 8 / 2019$ & 304 & 308 & & & & & & & & & & 314 & 303 \\
\hline $5 / 15 / 2019$ & 304 & 308 & & & & & & & & & & 315 & 302 \\
\hline $5 / 1 / 2019$ & 304 & 308 & & & & & & & & & & 313 & 303 \\
\hline $5 / 8 / 2019$ & 304 & 308 & & & & & & & & & & 314 & 303 \\
\hline $5 / 15 / 2019$ & 304 & 308 & & & & & & & & & & 315 & 302 \\
\hline $5 / 1 / 2019$ & 304 & 308 & & & & & & & & & & 313 & 303 \\
\hline $5 / 8 / 2019$ & 304 & 308 & & & & & & & & & & 314 & 303 \\
\hline $5 / 15 / 2019$ & 304 & 308 & & & & & & & & & & 315 & 302 \\
\hline
\end{tabular}




\section{Appendix E \\ MRM Pressure Release Data}




\begin{tabular}{|c|c|c|c|c|c|c|c|c|}
\hline \multicolumn{3}{|c|}{ Pressure Data from Executed Procedures } & \multirow{2}{*}{$\begin{array}{c}\text { Manifold } \\
\text { pressure } \\
\text { psia } \\
\end{array}$} & \multirow{2}{*}{$\begin{array}{l}\text { Man } \\
\text { Prel } \\
\text { psia } \\
\end{array}$} & \multirow{2}{*}{$\begin{array}{c}\begin{array}{c}\text { Man } \\
\text { rel }\end{array} \\
\text { psia } \\
\end{array}$} & \multirow{2}{*}{$\begin{array}{c}\text { Release } \\
\quad \mathrm{P} \\
\\
\text { psia } \\
\end{array}$} & \multirow{2}{*}{$\begin{array}{c}\begin{array}{c}\text { Calculation } \\
\text { P }\end{array} \\
\text { psia } \\
\end{array}$} & \multirow{2}{*}{$\begin{array}{c}\mathrm{CO} \mathrm{Pr} \\
\mathrm{Pa}\end{array}$} \\
\hline $\begin{array}{l}\text { Manifold } \\
\text { / Station }\end{array}$ & Date & Can ID & & & & & & \\
\hline A & $5 / 28 / 2014$ & $\mathrm{~L} 126, \mathrm{~L} 134$ & 26.8 & & & 10.99 & & 2523.1 \\
\hline A & $6 / 4 / 2014$ & L126, L134 & 20.28 & & & 8.29 & & 430.1 \\
\hline A & $6 / 11 / 2014$ & L126, L134 & 19.76 & & & 8.08 & & 367.9 \\
\hline $\mathrm{A} / 4$ & $6 / 17 / 2014$ & $\mathrm{~L} 126$ & & 2.03 & & 2.26 & 20.06 & 179.63 \\
\hline $\mathrm{A} / 4$ & $6 / 26 / 2014$ & L126 & & 2.03 & & 2.26 & 20.05 & 290.96 \\
\hline$A / 4$ & $7 / 2 / 2014$ & L126 & & 2.03 & & 2.23 & 20.06 & 230.9 \\
\hline $\mathrm{A} / 4$ & $7 / 9 / 2014$ & L126 & & 2.04 & & 2.25 & 20.05 & 309.97 \\
\hline$A / 4$ & $7 / 16 / 2014$ & L126 & & 2.03 & & 2.23 & 20.05 & 356.8 \\
\hline$C / 21$ & $4 / 27 / 2017$ & L126 & & 7.23 & 2.87 & 2.97 & 20.11 & 5443.7 \\
\hline$c / 21$ & $5 / 25 / 2017$ & L126 & & 6.28 & 2.52 & 2.53 & 20.09 & 684.21 \\
\hline $\mathrm{C} / 21$ & $6 / 21 / 2017$ & L126 & & 6.13 & 2.48 & 2.38 & 20.02 & 311.6 \\
\hline$C / 21$ & $7 / 25 / 2017$ & L126 & & 6.21 & 2.5 & 2.41 & 20.06 & 186.8 \\
\hline$C / 21$ & $8 / 24 / 2017$ & L126 & & 6.32 & 2.49 & 2.39 & 20 & 109.2 \\
\hline$C / 21$ & $11 / 6 / 2017$ & L126 & & 5.93 & 2.31 & 2.27 & 20.02 & 297.8 \\
\hline$C / 21$ & $1 / 10 / 2018$ & L126 & & 5.68 & 2.2 & 2.15 & 20.06 & 192.3 \\
\hline$c / 21$ & $3 / 5 / 2018$ & L126 & & 5.57 & 2.14 & 2.09 & 20.18 & 277.8 \\
\hline$c / 21$ & $4 / 4 / 2018$ & L126 & & 5.35 & 2.05 & 2.03 & 20.07 & 65.9 \\
\hline$c / 21$ & $5 / 2 / 2018$ & L126 & & 5.35 & 2.05 & 2.09 & 20.02 & 112.6 \\
\hline$c / 21$ & $6 / 6 / 2018$ & L126 & & 5.36 & 2.05 & 2.08 & 20.04 & 58.1 \\
\hline$C / 21$ & $7 / 11 / 2018$ & L126 & & 5.35 & 2.03 & 2.08 & 19.99 & 128.1 \\
\hline$c / 21$ & $8 / 7 / 2018$ & L126 & & 5.34 & 2.03 & 2.05 & 19.99 & 85.6 \\
\hline$c / 21$ & $9 / 6 / 2018$ & L126 & & 5.43 & 2.15 & 2.01 & 20.09 & 52.5 \\
\hline$c / 21$ & $10 / 4 / 2018$ & L126 & & 5.37 & 2.14 & 1.99 & 19.99 & 98.8 \\
\hline$C / 21$ & $11 / 12 / 2018$ & L126 & & 5.52 & 2.2 & 2.08 & 20.05 & 127 \\
\hline$c / 21$ & $12 / 12 / 2018$ & L126 & & 5.43 & 2.16 & 2.06 & 20.04 & 90.9 \\
\hline$C / 21$ & $1 / 9 / 2019$ & L126 & & 5.52 & 2.19 & 2.09 & 20.09 & 101 \\
\hline $\mathrm{A} / 3$ & $6 / 17 / 2014$ & L134 & & 1.97 & & 2.19 & 20.03 & 253.13 \\
\hline $\mathrm{A} / 3$ & $6 / 26 / 2014$ & L134 & & 1.98 & & 2.2 & 20.04 & 320.7 \\
\hline$A / 3$ & $7 / 2 / 2014$ & L134 & & 1.98 & & 2.17 & 20.04 & 286.5 \\
\hline $\mathrm{A} / 3$ & $7 / 9 / 2014$ & L134 & & 1.98 & & 2.2 & 20.05 & 332.67 \\
\hline$A / 3$ & $7 / 16 / 2014$ & $\mathrm{~L} 134$ & & 1.98 & & 2.17 & 20.05 & 356.8 \\
\hline$c / 22$ & $4 / 27 / 2017$ & L134 & & 7.14 & 2.82 & 2.92 & 20.17 & 4872 \\
\hline$c / 22$ & $5 / 25 / 2017$ & L134 & & 6.3 & 2.53 & 2.54 & 20.11 & 531.85 \\
\hline$C / 22$ & $6 / 21 / 2017$ & L134 & & 6.13 & 2.45 & 2.39 & 20.05 & 276.6 \\
\hline$c / 22$ & $7 / 25 / 2017$ & L134 & & 6.2 & 2.52 & 2.39 & 20.05 & 128.9 \\
\hline$c / 22$ & $8 / 24 / 2017$ & L134 & & 6.41 & 2.49 & 2.39 & 20.01 & 131.4 \\
\hline$C / 22$ & $11 / 6 / 2017$ & L134 & & 6 & 2.32 & 2.28 & 20.02 & 109 \\
\hline $\mathrm{C} / 22$ & $1 / 10 / 2018$ & L134 & & 5.56 & 2.14 & 2.05 & 20.06 & 130.6 \\
\hline$c / 22$ & $3 / 5 / 2018$ & $\mathrm{~L} 134$ & & 5.4 & 2.04 & 2.01 & 20.15 & 206.4 \\
\hline$c / 22$ & $4 / 4 / 2018$ & L134 & & 5.19 & 1.97 & 1.95 & 20.06 & 71.2 \\
\hline
\end{tabular}




\begin{tabular}{|c|c|c|c|c|c|c|c|c|}
\hline \multicolumn{3}{|c|}{ Pressure Data from Executed Procedures } & \multirow[t]{2}{*}{$\begin{array}{l}\text { Manifold } \\
\text { pressure }\end{array}$} & \multirow{2}{*}{$\begin{array}{l}\text { Man } \\
\text { Prel } \\
5.19 \\
\end{array}$} & \multirow{2}{*}{$\begin{array}{c}\begin{array}{c}\text { Man } \\
\text { rel }\end{array} \\
1.99 \\
\end{array}$} & \multirow{2}{*}{$\begin{array}{c}\text { Release } \\
\mathrm{P}\end{array}$} & \multirow{2}{*}{$\begin{array}{c}\text { Calculation } \\
\text { P }\end{array}$} & \multirow{2}{*}{$\begin{array}{c}\mathrm{CO} P r \\
127.32\end{array}$} \\
\hline $\mathrm{C} / 22$ & $5 / 2 / 2018$ & L134 & & & & & & \\
\hline$c / 22$ & $6 / 6 / 2018$ & L134 & & 5.2 & 1.97 & 2 & 20.04 & 57.8 \\
\hline $\mathrm{C} / 22$ & $7 / 11 / 2018$ & L134 & & 5.19 & 1.95 & 2.01 & 20 & 104.6 \\
\hline $\mathrm{C} / 22$ & $8 / 7 / 2018$ & $\mathrm{~L} 134$ & & 5.18 & 1.98 & 1.99 & 20 & 84.7 \\
\hline $\mathrm{C} / 22$ & $9 / 6 / 2018$ & L134 & & 5.27 & 2.08 & 1.94 & 20.07 & 70.4 \\
\hline$c / 22$ & $10 / 4 / 2018$ & $\mathrm{~L} 134$ & & 5.25 & 2.05 & 1.92 & 19.99 & 98.1 \\
\hline $\mathrm{C} / 22$ & $11 / 12 / 2018$ & L134 & & 5.36 & 2.12 & 2.01 & 20.05 & 130.3 \\
\hline $\mathrm{C} / 22$ & $12 / 12 / 2018$ & L134 & & 5.3 & 2.08 & 2 & 20.05 & 93.1 \\
\hline$c / 22$ & $1 / 9 / 2019$ & L134 & & 5.35 & 2.12 & 2.03 & 20.09 & 100 \\
\hline B & $12 / 10 / 2014$ & L1307, L1308 & 23.55 & & & 9.76 & & 2598.00 \\
\hline B & $12 / 17 / 2014$ & L1307, L1309 & 20.35 & & & 8.41 & & 208.80 \\
\hline$B / 11$ & $12 / 22 / 2014$ & L1307 & & & 1.92 & 2.22 & 20.06 & 150.36 \\
\hline$B / 11$ & $1 / 7 / 2015$ & L1307 & & & 1.94 & 2.23 & 20.06 & 198.20 \\
\hline $\mathrm{B} / 11$ & $1 / 14 / 2015$ & L1307 & & & 1.92 & 2.22 & 20.00 & 193.70 \\
\hline $\mathrm{B} / 11$ & $1 / 21 / 2015$ & L1307 & & & 1.92 & 2.21 & 20.05 & 150.46 \\
\hline$B / 11$ & $1 / 28 / 2015$ & L1307 & & & 1.93 & 2.20 & 20.07 & 130.20 \\
\hline$C / 25$ & $4 / 27 / 2017$ & L1307 & & 6.83 & 2.74 & 2.82 & 20.18 & 2071.90 \\
\hline$c / 25$ & $5 / 25 / 2017$ & L1307 & & 6.37 & 2.58 & 2.60 & 20.12 & 310.15 \\
\hline$C / 25$ & $6 / 21 / 2017$ & L1307 & & 6.26 & 2.54 & 2.45 & 20.04 & 129.80 \\
\hline$C / 25$ & $7 / 25 / 2017$ & L1307 & & 6.36 & 2.60 & 2.48 & 20.05 & 93.10 \\
\hline$c / 25$ & $8 / 24 / 2017$ & L1307 & & 6.57 & 2.59 & 2.49 & 20.02 & 102.50 \\
\hline$C / 25$ & $11 / 6 / 2017$ & L1307 & & 6.13 & 2.41 & 2.34 & 20.02 & 390.40 \\
\hline$c / 25$ & $1 / 10 / 2018$ & L1307 & & 5.87 & 2.28 & 2.22 & 20.02 & 431.40 \\
\hline$c / 25$ & $3 / 5 / 2018$ & L1307 & & 5.71 & 2.20 & 2.17 & 20.16 & 293.50 \\
\hline$c / 25$ & $4 / 4 / 2018$ & L1307 & & 5.52 & 2.11 & 2.09 & 20.06 & 112.00 \\
\hline$C / 25$ & $5 / 2 / 2018$ & L1307 & & 5.49 & 2.10 & 2.15 & 20.01 & 127.32 \\
\hline$C / 25$ & $6 / 6 / 2018$ & L1307 & & 5.51 & 2.10 & 2.15 & 20.01 & 43.10 \\
\hline$c / 25$ & $7 / 11 / 2018$ & L1307 & & 5.48 & 2.08 & 2.15 & 19.99 & 110.20 \\
\hline$C / 25$ & $8 / 7 / 2018$ & L1307 & & 5.46 & 2.08 & 2.09 & 19.99 & 101.20 \\
\hline$c / 25$ & $9 / 6 / 2018$ & L1307 & & 5.54 & 2.20 & 2.06 & 20.07 & 76.70 \\
\hline$C / 25$ & $10 / 4 / 2018$ & L1307 & & 5.52 & 2.19 & 2.05 & 19.99 & 110.60 \\
\hline$c / 25$ & $11 / 12 / 2018$ & L1307 & & 5.63 & 2.25 & 2.14 & 20.06 & 136.10 \\
\hline$C / 25$ & $12 / 12 / 2018$ & L1307 & & 5.56 & 2.21 & 2.11 & 20.05 & 94.60 \\
\hline$c / 25$ & $1 / 9 / 2019$ & L1307 & & 5.63 & 2.25 & 2.15 & 20.07 & 98.80 \\
\hline$B / 12$ & $12 / 22 / 2014$ & L1308 & & & 1.99 & 2.28 & 20.02 & 218.93 \\
\hline$B / 12$ & $1 / 7 / 2015$ & L1308 & & & 2.04 & 2.33 & 20.04 & 1627.00 \\
\hline$B / 12$ & $1 / 14 / 2015$ & L1308 & & & 1.97 & 2.27 & 19.99 & 254.20 \\
\hline $\mathrm{B} / 12$ & $1 / 21 / 2015$ & L1308 & & & 1.99 & 2.27 & 20.02 & 170.45 \\
\hline$B / 12$ & $1 / 28 / 2015$ & L1308 & & & 1.99 & 2.25 & 20.09 & 157.90 \\
\hline$c / 26$ & $4 / 27 / 2017$ & L1308 & & 6.97 & 2.80 & 2.88 & 20.23 & 4003.20 \\
\hline$c / 26$ & $5 / 25 / 2017$ & L1308 & & 6.41 & 2.60 & 2.61 & 20.12 & 459.60 \\
\hline
\end{tabular}




\begin{tabular}{|c|c|c|c|c|c|c|c|c|}
\hline \multicolumn{3}{|c|}{ Pressure Data from Executed Procedures } & \multirow[t]{2}{*}{$\begin{array}{l}\text { Manifold } \\
\text { pressure }\end{array}$} & \multirow{2}{*}{$\begin{array}{l}\text { Man } \\
\text { Prel } \\
6.30 \\
\end{array}$} & \multirow{2}{*}{$\begin{array}{c}\begin{array}{c}\text { Man } \\
\text { rel }\end{array} \\
2.55 \\
\end{array}$} & \multirow{2}{*}{$\begin{array}{c}\text { Release } \\
\mathrm{P}\end{array}$} & \multirow{2}{*}{$\begin{array}{c}\text { Calculation } \\
\text { P }\end{array}$} & \multirow{2}{*}{$\begin{array}{l}\mathrm{CO} P r \\
182.70\end{array}$} \\
\hline $\mathrm{C} / 26$ & $6 / 21 / 2017$ & L1308 & & & & & & \\
\hline$c / 26$ & $7 / 25 / 2017$ & L1308 & & 6.39 & 2.60 & 2.49 & 20.06 & 131.20 \\
\hline$c / 26$ & $8 / 24 / 2017$ & L1308 & & 6.68 & 2.60 & 2.52 & 20.05 & 117.30 \\
\hline$c / 26$ & $11 / 6 / 2017$ & L1308 & & 6.18 & 2.42 & 2.37 & 20.04 & 98.90 \\
\hline$c / 26$ & $1 / 10 / 2018$ & L1308 & & 5.78 & 2.22 & 2.17 & 20.09 & 122.00 \\
\hline$c / 26$ & $3 / 5 / 2018$ & L1308 & & 5.56 & 2.14 & 2.09 & 20.17 & 141.70 \\
\hline$c / 26$ & $4 / 4 / 2018$ & L1308 & & 5.34 & 2.04 & 2.01 & 20.02 & 60.70 \\
\hline $\mathrm{C} / 26$ & $5 / 2 / 2018$ & L1308 & & 5.35 & 2.05 & 2.08 & 20.01 & 97.06 \\
\hline$c / 26$ & $6 / 6 / 2018$ & L1308 & & 5.36 & 2.05 & 2.08 & 20.02 & 41.70 \\
\hline$c / 26$ & $7 / 11 / 2018$ & L1308 & & 5.35 & 2.03 & 2.08 & 19.99 & 56.60 \\
\hline$c / 26$ & $8 / 7 / 2018$ & L1308 & & 5.31 & 2.03 & 2.05 & 19.99 & 89.20 \\
\hline$c / 26$ & 9/6/2018 & L1308 & & 5.40 & 2.10 & 1.99 & 20.06 & 63.20 \\
\hline$c / 26$ & $10 / 4 / 2018$ & L1308 & & 5.37 & 2.14 & 1.99 & 19.99 & 101.20 \\
\hline$C / 26$ & $11 / 12 / 2018$ & L1308 & & 5.51 & 2.19 & 2.06 & 20.06 & 113.20 \\
\hline$C / 26$ & $12 / 12 / 2018$ & L1308 & & 5.42 & 2.16 & 2.06 & 20.06 & 84.20 \\
\hline$c / 26$ & $1 / 9 / 2019$ & L1308 & & 5.43 & 2.19 & 2.08 & 20.06 & 94.60 \\
\hline $\mathrm{A} / 3$ & $5 / 31 / 2017$ & L1303 & 21.17 & & 7.42 & 7.45 & 21.16 & 2896.16 \\
\hline$A / 3$ & $6 / 8 / 2017$ & L1303 & 20.01 & & 7.02 & 7 & 20.01 & 221.7 \\
\hline $\mathrm{A} / 3$ & $6 / 14 / 2017$ & L1303 & 19.96 & & 7.03 & 6.92 & 19.98 & 135.7 \\
\hline $\mathrm{A} / 3$ & $6 / 21 / 2017$ & L1303 & & 6.78 & 2.42 & 2.36 & 20.04 & 155.7 \\
\hline$A / 3$ & $6 / 28 / 2017$ & L1303 & & 6.78 & 2.43 & 2.34 & 20.02 & 117.1 \\
\hline $\mathrm{A} / 3$ & $7 / 6 / 2017$ & L1303 & & 6.78 & 2.42 & 2.37 & 20.01 & 123 \\
\hline $\mathrm{A} / 3$ & $7 / 12 / 2017$ & L1303 & & 6.79 & 2.43 & 2.38 & 20.05 & 115.6 \\
\hline $\mathrm{A} / 3$ & $7 / 19 / 2017$ & L1303 & & 6.76 & 2.42 & 2.34 & 20.01 & 129.6 \\
\hline $\mathrm{A} / 3$ & $7 / 25 / 2017$ & L1303 & & 6.76 & 2.42 & 2.37 & 20.06 & 106.7 \\
\hline $\mathrm{A} / 3$ & $8 / 2 / 2017$ & L1303 & & 6.78 & 2.43 & 2.38 & 20.02 & 108.5 \\
\hline$A / 3$ & $8 / 9 / 2017$ & L1303 & & 6.75 & 2.42 & 2.33 & 20.02 & 118.2 \\
\hline $\mathrm{A} / 3$ & $8 / 16 / 2017$ & L1303 & & 6.78 & 2.42 & 2.36 & 20.05 & 115.5 \\
\hline$A / 3$ & $8 / 24 / 2017$ & L1303 & & 6.48 & 2.32 & 2.26 & 20 & 116.4 \\
\hline $\mathrm{A} / 3$ & $11 / 6 / 2017$ & L1303 & & 8.32 & 2.95 & 2.89 & 20.04 & 12952 \\
\hline $\mathrm{A} / 3$ & $12 / 6 / 2017$ & L1303 & & 6.65 & 2.38 & 2.32 & 20.07 & 700.6 \\
\hline$A / 3$ & $1 / 10 / 2018$ & L1303 & & 6.62 & 2.34 & 2.28 & 20.05 & 346.7 \\
\hline $\mathrm{A} / 3$ & $2 / 7 / 2018$ & L1303 & & 6.67 & 2.36 & 2.26 & 20.03 & 280.6 \\
\hline$A / 3$ & $3 / 5 / 2018$ & L1303 & & 6.26 & 2.26 & 2.22 & 20 & 124.3 \\
\hline$C / 27$ & $4 / 4 / 2018$ & L1303 & & 6.84 & 2.65 & 2.61 & 20.09 & 63.1 \\
\hline$C / 27$ & $5 / 2 / 2018$ & L1303 & & 5.64 & 2.2 & 2.21 & 20.01 & 101.5 \\
\hline$C / 27$ & $6 / 6 / 2018$ & L1303 & & 5.64 & 2.17 & 2.22 & 20.02 & 41.7 \\
\hline$C / 27$ & $7 / 11 / 2018$ & L1303 & & 5.62 & 2.16 & 2.21 & 20 & 66.8 \\
\hline$c / 27$ & $8 / 7 / 2018$ & L1303 & & 5.63 & 2.17 & 2.2 & 20 & 91.2 \\
\hline$C / 27$ & $9 / 6 / 2018$ & L1303 & & 5.69 & 2.27 & 2.16 & 20.07 & 76.4 \\
\hline$c / 27$ & $10 / 4 / 2018$ & L1303 & & 5.57 & 2.27 & 2.14 & 19.98 & 103.3 \\
\hline
\end{tabular}




\begin{tabular}{|c|c|c|c|c|c|c|c|c|}
\hline \multicolumn{3}{|c|}{ Pressure Data from Executed Procedures } & \multirow[t]{2}{*}{$\begin{array}{l}\text { Manifold } \\
\text { pressure }\end{array}$} & \multirow{2}{*}{$\begin{array}{l}\text { Man } \\
\text { Prel } \\
5.79\end{array}$} & \multirow{2}{*}{$\begin{array}{c}\begin{array}{c}\text { Man } \\
\text { rel }\end{array} \\
2.32 \\
\end{array}$} & \multirow{2}{*}{$\begin{array}{c}\text { Release } \\
\mathrm{P}\end{array}$} & \multirow{2}{*}{$\begin{array}{c}\text { Calculation } \\
\text { P }\end{array}$} & \multirow{2}{*}{$\begin{array}{c}\mathrm{CO} \mathrm{Pr} \\
117 \\
\end{array}$} \\
\hline $\mathrm{C} / 27$ & $11 / 12 / 2018$ & L1303 & & & & & & \\
\hline$c / 27$ & $12 / 12 / 2018$ & L1303 & & 5.73 & 2.3 & 2.2 & 20.06 & 88.4 \\
\hline$C / 27$ & $1 / 9 / 2019$ & L1303 & & 5.8 & 2.33 & 2.23 & 20.06 & 89.8 \\
\hline $\mathrm{A} / 3$ & $4 / 25 / 2018$ & L1701 & 24.4 & & 8.32 & 8.32 & 24.4 & 340.88 \\
\hline $\mathrm{A} / 3$ & $5 / 2 / 2018$ & L1701 & 20.12 & & 6.84 & 6.86 & 20.12 & 197.54 \\
\hline $\mathrm{A} / 3$ & $5 / 9 / 2018$ & L1701 & & 6.23 & 2.14 & 2.15 & 20.06 & 94.2 \\
\hline $\mathrm{A} / 3$ & $5 / 16 / 2018$ & L1701 & & 6.25 & 2.15 & 2.16 & 20.12 & 96.95 \\
\hline $\mathrm{A} / 3$ & $5 / 22 / 2018$ & L1701 & & 6.21 & 2.14 & 2.15 & 20.05 & 253.8 \\
\hline$A / 3$ & $5 / 30 / 2018$ & L1701 & & 6.2 & 2.12 & 2.15 & 20.02 & 168.5 \\
\hline $\mathrm{A} / 3$ & $6 / 6 / 2018$ & L1701 & & 5.95 & 2.04 & 2.06 & 20.04 & 83.6 \\
\hline $\mathrm{A} / 3$ & $7 / 11 / 2018$ & L1701 & & 6.24 & 2.16 & 2.12 & 20.11 & 58.8 \\
\hline $\mathrm{A} / 3$ & $8 / 7 / 2018$ & L1701 & & 6.15 & 2.12 & 2.1 & 20.11 & 128.1 \\
\hline$A / 3$ & $9 / 6 / 2018$ & L1701 & & 6.13 & 2.14 & 2.05 & 20.07 & 53 \\
\hline $\mathrm{A} / 3$ & $10 / 4 / 2018$ & L1701 & & 6.11 & 2.12 & 2.03 & 19.99 & 101.8 \\
\hline $\mathrm{A} / 3$ & $11 / 12 / 2018$ & L1701 & & 6.24 & 2.19 & 2.14 & 20.07 & 189.5 \\
\hline$A / 3$ & $12 / 12 / 2018$ & L1701 & & 6.13 & 2.16 & 2.09 & 20.04 & 223.1 \\
\hline $\mathrm{A} / 3$ & $1 / 9 / 2019$ & L1701 & & 6.18 & 2.15 & 2.15 & 20.12 & 155.1 \\
\hline $\mathrm{B}$ & $9 / 5 / 2018$ & L1301, L1306, L1311 & 28.41 & & 13.22 & 13.33 & 28.42 & 2593.5 \\
\hline B & $9 / 13 / 2018$ & L1301, L1306, L1311 & 20.1 & & 9.26 & 9.43 & 20.1 & 240.6 \\
\hline$B / 11$ & $9 / 20 / 2018$ & L1301 & & 5.81 & 1.87 & 2.06 & 19.99 & 180.5 \\
\hline$B / 11$ & $9 / 27 / 2018$ & L1301 & & 5.78 & 1.83 & 2.1 & 19.99 & 206.7 \\
\hline $\mathrm{B} / 11$ & $10 / 4 / 2018$ & L1301 & & 5.78 & 1.81 & 2.06 & 19.99 & 183.7 \\
\hline $\mathrm{B} / 11$ & $10 / 11 / 2018$ & L1301 & & 5.48 & 1.68 & 1.97 & 20.01 & 162.7 \\
\hline $\mathrm{B} / 11$ & $11 / 13 / 2018$ & L1301 & & 5.59 & 1.72 & 2.08 & 19.74 & 50.5 \\
\hline$B / 11$ & $12 / 12 / 2018$ & L1301 & & 5.67 & 1.73 & 2.09 & 20.09 & 141.2 \\
\hline $\mathrm{B} / 11$ & $1 / 9 / 2019$ & L1301 & & 5.76 & 1.79 & 2.11 & 20.09 & 108.8 \\
\hline$B / 13$ & $9 / 20 / 2018$ & L1306 & & 6.35 & 2.11 & 2.33 & 19.99 & 182.7 \\
\hline$B / 13$ & $9 / 27 / 2018$ & L1306 & & 6.37 & 2.08 & 2.37 & 19.99 & 255.3 \\
\hline$B / 13$ & $10 / 4 / 2018$ & L1306 & & 6.3 & 2.04 & 2.31 & 19.99 & 143.5 \\
\hline$B / 13$ & $10 / 11 / 2018$ & L1306 & & 6.01 & 1.84 & 2.12 & 20.02 & 163.9 \\
\hline $\mathrm{B} / 13$ & $11 / 13 / 2018$ & L1306 & & 6.04 & 1.93 & 2.28 & 19.74 & 142.5 \\
\hline$B / 13$ & $12 / 12 / 2018$ & L1306 & & 6.12 & 1.95 & 2.31 & 20.09 & 213.7 \\
\hline$B / 13$ & $1 / 9 / 2019$ & L1306 & & 6.23 & 2.01 & 2.34 & 20.09 & 142.6 \\
\hline$B / 15$ & $9 / 20 / 2018$ & L1311 & & 6.36 & 2.11 & 2.33 & 20.01 & 148.5 \\
\hline$B / 15$ & $9 / 27 / 2018$ & L1311 & & 6.35 & 2.09 & 2.34 & 20.01 & 170.7 \\
\hline$B / 15$ & $10 / 4 / 2018$ & L1311 & & 6.3 & 2.05 & 2.31 & 20.01 & 141.9 \\
\hline$B / 15$ & $10 / 11 / 2018$ & L1311 & & 5.91 & 1.89 & 2.16 & 20.01 & 151.7 \\
\hline $\mathrm{B} / 15$ & $11 / 13 / 2018$ & L1311 & & 5.93 & 1.88 & 2.25 & 19.74 & 58.8 \\
\hline $\mathrm{B} / 15$ & $12 / 12 / 2018$ & L1311 & & 6.02 & 1.9 & 2.27 & 20.09 & 125.4 \\
\hline $\mathrm{B} / 15$ & $1 / 9 / 2019$ & L1311 & & 6.12 & 1.98 & 2.31 & 20.09 & 117.6 \\
\hline A & $4 / 24 / 2019$ & $\begin{array}{l}\text { L1702, L1704, L1707, } \\
\text { L1708 }\end{array}$ & 20.48 & & 9.44 & 9.37 & 20.48 & 243.8 \\
\hline
\end{tabular}




\begin{tabular}{|c|c|c|c|c|c|c|c|c|}
\hline \multicolumn{3}{|c|}{ Pressure Data from Executed Procedures } & \multirow[t]{2}{*}{$\begin{array}{l}\text { Manifold } \\
\text { pressure }\end{array}$} & \multirow{2}{*}{$\begin{array}{l}\text { Man } \\
\text { Prel } \\
5.32 \\
\end{array}$} & \multirow{2}{*}{$\begin{array}{c}\text { Man } \\
\text { rel }\end{array}$} & \multirow{2}{*}{$\begin{array}{c}\text { Release } \\
\mathrm{P}\end{array}$} & \multirow{2}{*}{$\begin{array}{c}\text { Calculation } \\
\text { P }\end{array}$} & \multirow{2}{*}{$\begin{array}{c}\text { CO Pr } \\
146.02 \\
\end{array}$} \\
\hline A / 3 & $5 / 1 / 2019$ & L1702 & & & & & & \\
\hline A / 3 & $5 / 8 / 2019$ & L1702 & & 5.34 & 1.79 & 1.72 & 20.11 & 121.7 \\
\hline A / 3 & $5 / 15 / 2019$ & L1702 & & 5.31 & 1.77 & 1.73 & 20.07 & 127.7 \\
\hline A / 4 & $5 / 1 / 2019$ & L1704 & & 5.35 & 1.78 & 1.67 & 20.006 & 126.18 \\
\hline A / 4 & $5 / 8 / 2019$ & L1704 & & 5.38 & 1.82 & 1.75 & 20.01 & 109.8 \\
\hline A / 4 & $5 / 15 / 2019$ & L1704 & & 5.38 & 1.81 & 1.76 & 20.09 & 143.8 \\
\hline$A / 7$ & $5 / 1 / 2019$ & L1707 & & 5.25 & 1.76 & 1.65 & 20.1 & 111.86 \\
\hline$A / 7$ & $5 / 8 / 2019$ & L1707 & & 5.3 & 1.78 & 1.71 & 20.01 & 121.1 \\
\hline A / 7 & $5 / 15 / 2019$ & L1707 & & 5.3 & 1.77 & 1.73 & 20.04 & 123.6 \\
\hline A / 8 & $5 / 1 / 2019$ & L1708 & & 5.38 & 1.82 & 1.7 & 20.06 & 129.42 \\
\hline A / 8 & $5 / 8 / 2019$ & L1708 & & 5.41 & 1.82 & 1.75 & 20.05 & 121.4 \\
\hline A / 8 & $5 / 15 / 2019$ & L1708 & & 5.4 & 1.81 & 1.77 & 20.07 & 111.8 \\
\hline
\end{tabular}




\section{Appendix F MRM Can L1303 Reduction Calculation using the RGA Vacuum Pressure}




\begin{tabular}{|c|c|c|c|c|c|c|c|c|c|c|c|c|c|}
\hline \multirow[t]{2}{*}{ Method } & \multicolumn{2}{|c|}{ RGA Pressure } & \multicolumn{6}{|c|}{ Data from executed procedures } & \multicolumn{5}{|c|}{ Partial Pressure calculations } \\
\hline & & & $\begin{array}{l}\text { Manifold } \\
\text { pressure }\end{array}$ & $\begin{array}{c}\text { Man Prel } \\
A, B \text { or } C\end{array}$ & $\begin{array}{l}\text { Man rel } \\
A, B \text { or } C\end{array}$ & $\begin{array}{c}\text { Release } \\
\text { pres }\end{array}$ & Calc $\mathrm{Pr}$ & $\mathrm{CO} \mathrm{Pr}$ & $\begin{array}{l}\text { Calc CO } \\
\text { (tot P) }\end{array}$ & $\begin{array}{l}\text { Calc He } \\
\text { (tot P) }\end{array}$ & $\begin{array}{c}\text { Calc CO2 } \\
\text { (tot P) }\end{array}$ & $\begin{array}{l}\mathrm{CO}+\mathrm{He} \\
\text { (tot } \mathrm{P})\end{array}$ & $\begin{array}{c}\text { Tmax } \\
\text { (Tot P) }\end{array}$ \\
\hline Date & $\begin{array}{l}\text { Manifold } \\
\text { / Station }\end{array}$ & $\begin{array}{l}\text { MRM Can } \\
\text { Sampled }\end{array}$ & psia & psia & psia & psia & psia & $\mathrm{Pa}$ & $\mathrm{Pa}$ & $\mathrm{Pa}$ & $\mathrm{Pa}$ & psia & $\mathrm{K}$ \\
\hline $5 / 31 / 2017$ & $\mathrm{~A} / 3$ & L1303 & 21.17 & & 7.42 & 7.45 & 21.16 & 2896 & 2897 & 1699 & 14 & 1 & 1068 \\
\hline $6 / 8 / 2017$ & $\mathrm{~A} / 3$ & $\mathrm{~L} 1303$ & 20.01 & & 7.02 & 7.00 & 20.01 & 222 & 222 & 270 & 21 & 0 & 830 \\
\hline $6 / 14 / 2017$ & $\mathrm{~A} / 3$ & L1303 & 19.96 & & 7.03 & 6.92 & 19.98 & 136 & 136 & 193 & 18 & 0 & 802 \\
\hline $6 / 21 / 2017$ & $\mathrm{~A} / 3$ & L1303 & & 6.78 & 2.42 & 2.36 & 20.04 & 156 & 156 & 779 & 22 & 0 & 806 \\
\hline $6 / 28 / 2017$ & $\mathrm{~A} / 3$ & $\mathrm{~L} 1303$ & & 6.78 & 2.43 & 2.34 & 20.02 & 117 & 117 & 669 & 13 & 0 & 804 \\
\hline $7 / 6 / 2017$ & $\mathrm{~A} / 3$ & L1303 & & 6.78 & 2.42 & 2.37 & 20.01 & 123 & 123 & 768 & 17 & 0 & 798 \\
\hline $7 / 12 / 2017$ & $A / 3$ & L1303 & & 6.79 & 2.43 & 2.38 & 20.05 & 116 & 116 & 642 & 16 & 0 & 797 \\
\hline $7 / 19 / 2017$ & $\mathrm{~A} / 3$ & L1303 & & 6.76 & 2.42 & 2.34 & 20.01 & 130 & 130 & 745 & 24 & 0 & 792 \\
\hline $7 / 25 / 2017$ & $\mathrm{~A} / 3$ & L1303 & & 6.76 & 2.42 & 2.37 & 20.06 & 107 & 107 & 617 & 8 & 0 & 814 \\
\hline $8 / 2 / 2017$ & $\mathrm{~A} / 3$ & L1303 & & 6.78 & 2.43 & 2.38 & 20.02 & 109 & 108 & 876 & 13 & 0 & 798 \\
\hline $8 / 9 / 2017$ & $\mathrm{~A} / 3$ & L1303 & & 6.75 & 2.42 & 2.33 & 20.02 & 118 & 118 & 824 & 16 & 0 & 798 \\
\hline $8 / 16 / 2017$ & $A / 3$ & L1303 & & 6.78 & 2.42 & 2.36 & 20.05 & 116 & 115 & 789 & 13 & 0 & 804 \\
\hline $8 / 24 / 2017$ & $\mathrm{~A} / 3$ & L1303 & & 6.48 & 2.32 & 2.26 & 20.00 & 116 & 116 & 859 & 12 & 0 & 807 \\
\hline $11 / 6 / 2017$ & $\mathrm{~A} / 3$ & L1303 & & 8.32 & 2.95 & 2.89 & 20.04 & 12952 & 12948 & 4839 & 21 & 3 & 1235 \\
\hline $12 / 6 / 2017$ & $A / 3$ & L1303 & & 6.65 & 2.38 & 2.32 & 20.07 & 701 & 700 & 3319 & 33 & 1 & 897 \\
\hline $1 / 10 / 2018$ & $\mathrm{~A} / 3$ & L1303 & & 6.62 & 2.34 & 2.28 & 20.05 & 347 & 347 & 4092 & 44 & 1 & 835 \\
\hline $2 / 7 / 2018$ & $\mathrm{~A} / 3$ & L1303 & & 6.67 & 2.36 & 2.26 & 20.03 & 281 & 280 & 3289 & 31 & 1 & 832 \\
\hline $3 / 5 / 2018$ & $A / 3$ & L1303 & & 6.26 & 2.26 & 2.22 & 20.00 & 124 & 124 & 2228 & 9 & 0 & 818 \\
\hline $4 / 4 / 2018$ & $\mathrm{C} / 27$ & L1303 & & 6.84 & 2.65 & 2.61 & 20.09 & 63 & 63 & 1537 & 6 & 0 & 790 \\
\hline $5 / 2 / 2018$ & $C / 27$ & L1303 & & 5.64 & 2.20 & 2.21 & 20.01 & 102 & 101 & 2521 & 8 & 0 & 812 \\
\hline $6 / 6 / 2018$ & $\mathrm{C} / 27$ & L1303 & & 5.64 & 2.17 & 2.22 & 20.02 & 42 & 42 & 1596 & 3 & 0 & 784 \\
\hline $7 / 11 / 2018$ & $\mathrm{C} / 27$ & L1303 & & 5.62 & 2.16 & 2.21 & 20.00 & 67 & 67 & 2731 & 7 & 0 & 790 \\
\hline $8 / 7 / 2018$ & $\mathrm{C} / 27$ & L1303 & & 5.63 & 2.17 & 2.20 & 20.00 & 91 & 91 & 2386 & 5 & 0 & 816 \\
\hline $9 / 6 / 2018$ & $\mathrm{C} / 27$ & L1303 & & 5.69 & 2.27 & 2.16 & 20.07 & 76 & 76 & 1987 & 3 & 0 & 822 \\
\hline $10 / 4 / 2018$ & $\mathrm{C} / 27$ & L1303 & & 5.57 & 2.27 & 2.14 & 19.98 & 103 & 103 & 2774 & 7 & 0 & 816 \\
\hline $11 / 12 / 2018$ & $\mathrm{C} / 27$ & L1303 & & 5.79 & 2.32 & 2.21 & 20.06 & 117 & 117 & 4312 & 9 & 1 & 814 \\
\hline $12 / 12 / 2018$ & $\mathrm{C} / 27$ & L1303 & & 5.73 & 2.30 & 2.20 & 20.06 & 88 & 88 & 2333 & 3 & 0 & 829 \\
\hline $1 / 9 / 2019$ & $\mathrm{C} / 27$ & L1303 & & 5.80 & 2.33 & 2.23 & 20.06 & 90 & 90 & 1706 & 5 & 0 & 819 \\
\hline
\end{tabular}




\begin{tabular}{|c|c|c|c|c|c|c|c|c|c|c|c|c|c|c|c|}
\hline \multicolumn{4}{|c|}{ Release Pressures } & \multicolumn{5}{|c|}{ Can pressure - double rel. } & \multicolumn{7}{|c|}{ Reduction Calculation } \\
\hline $\begin{array}{l}\text { Manifold } \\
\text { pressure }\end{array}$ & $\begin{array}{c}\text { Man } \\
\text { Prel } \\
\mathrm{A}, \mathrm{B} \text { or } \mathrm{C}\end{array}$ & $\begin{array}{c}\text { Man rel } \\
\text { A, B or } \\
\text { C }\end{array}$ & $\begin{array}{c}\text { Release } \\
\text { pres }\end{array}$ & $\begin{array}{l}\text { Variable } \\
(\mathrm{T} / \mathrm{V})\end{array}$ & $\begin{array}{l}\mathrm{N} \text { (MRM } \\
\text { can) }\end{array}$ & $\mathrm{P}$ (calc) & $\mathrm{P}$ (calc) & $\begin{array}{l}\text { Can } \\
\text { Temp }\end{array}$ & $\begin{array}{c}\# \text { mol } \\
\text { gas }\end{array}$ & $\# \mathrm{~mol} \mathrm{CO}$ & $\begin{array}{c}\# \mathrm{~mol} \\
\mathrm{CO} 2\end{array}$ & $\begin{array}{c}\text { \#mol of } \\
\text { "O" }\end{array}$ & $\begin{array}{c}\mathrm{O} 2 \\
\text { portion } \\
\text { PuO2 }\end{array}$ & PuOx & $\begin{array}{l}\text { Ratio of } \\
\text { mol calc }\end{array}$ \\
\hline $\mathrm{Pa}$ & $\mathrm{Pa}$ & $\mathrm{Pa}$ & $\mathrm{Pa}$ & & & $\mathrm{Pa}$ & psia & $\mathrm{K}$ & $\mathrm{mol}$ & & & & $\mathrm{mol}$ & & \\
\hline 145922 & 0 & 51145 & 51352 & & & & & 558 & $1.65 \mathrm{E}-01$ & $3.27 \mathrm{E}-03$ & 1.60E-05 & $3.31 \mathrm{E}-03$ & 4.47 & 1.9985 & \\
\hline 137926 & 0 & 48388 & 48250 & & & & & 563 & $1.56 \mathrm{E}-01$ & $2.51 \mathrm{E}-04$ & $2.36 \mathrm{E}-05$ & $2.98 \mathrm{E}-04$ & 4.47 & 1.9984 & \\
\hline 137581 & 0 & 48457 & 47699 & & & & & 561 & $1.56 \mathrm{E}-01$ & $1.53 \mathrm{E}-04$ & 2.07E-05 & $1.95 \mathrm{E}-04$ & 4.47 & 1.9983 & \\
\hline 0 & 46734 & 16681 & 16267 & 294329 & $5.28 \mathrm{E}-02$ & 129315 & 18.76 & 559 & $5.15 \mathrm{E}-02$ & $5.81 \mathrm{E}-05$ & $8.10 \mathrm{E}-06$ & 7.43E-05 & 4.47 & 1.9983 & 1.03 \\
\hline 0 & 46734 & 16750 & 16129 & 289176 & $5.32 \mathrm{E}-02$ & 127869 & 18.55 & 558 & $5.11 \mathrm{E}-02$ & 4.33E-05 & 4.86E-06 & $5.31 \mathrm{E}-05$ & 4.47 & 1.9982 & 1.04 \\
\hline 0 & 46734 & 16681 & 16336 & 294329 & $5.28 \mathrm{E}-02$ & 129315 & 18.76 & 558 & $5.17 \mathrm{E}-02$ & 4.61E-05 & $6.40 \mathrm{E}-06$ & $5.89 \mathrm{E}-05$ & 4.47 & 1.9982 & 1.02 \\
\hline 0 & 46803 & 16750 & 16405 & 291002 & $5.31 \mathrm{E}-02$ & 128570 & 18.65 & 558 & $5.20 \mathrm{E}-02$ & $4.35 \mathrm{E}-05$ & 5.99E-06 & $5.54 \mathrm{E}-05$ & 4.47 & 1.9982 & 1.02 \\
\hline 0 & 46596 & 16681 & 16129 & 290623 & $5.29 \mathrm{E}-02$ & 127896 & 18.55 & 560 & $5.11 \mathrm{E}-02$ & 4.80E-05 & $8.75 \mathrm{E}-06$ & $6.55 \mathrm{E}-05$ & 4.47 & 1.9982 & 1.04 \\
\hline 0 & 46596 & 16681 & 16336 & 290623 & $5.29 \mathrm{E}-02$ & 127896 & 18.55 & 559 & $5.17 \mathrm{E}-02$ & $3.99 \mathrm{E}-05$ & $2.96 \mathrm{E}-06$ & $4.58 \mathrm{E}-05$ & 4.47 & 1.9981 & 1.02 \\
\hline 0 & 46734 & 16750 & 16405 & 289176 & $5.32 \mathrm{E}-02$ & 127869 & 18.55 & 559 & $5.20 \mathrm{E}-02$ & $4.08 \mathrm{E}-05$ & 5.00E-06 & $5.08 \mathrm{E}-05$ & 4.47 & 1.9981 & 1.02 \\
\hline 0 & 46527 & 16681 & 16060 & 288793 & $5.30 \mathrm{E}-02$ & 127196 & 18.45 & 558 & 5.09E-02 & 4.36E-05 & 5.93E-06 & 5.54E-05 & 4.47 & 1.9981 & 1.04 \\
\hline 0 & 46734 & 16681 & 16267 & 294329 & $5.28 \mathrm{E}-02$ & 129315 & 18.76 & 559 & $5.15 \mathrm{E}-02$ & $4.31 \mathrm{E}-05$ & $4.76 \mathrm{E}-06$ & $5.26 \mathrm{E}-05$ & 4.47 & 1.9981 & 1.03 \\
\hline 0 & 44666 & 15991 & 15578 & 290497 & 5.07E-02 & 122565 & 17.78 & 559 & 4.93E-02 & 4.16E-05 & 4.14E-06 & 4.99E-05 & 4.47 & 1.9980 & 1.03 \\
\hline 0 & 57349 & 20334 & 19920 & 302934 & $6.42 \mathrm{E}-02$ & 161650 & 23.45 & 558 & $6.31 \mathrm{E}-02$ & $5.91 \mathrm{E}-03$ & $9.55 \mathrm{E}-06$ & 5.93E-03 & 4.46 & 1.9954 & 1.02 \\
\hline 0 & 45838 & 16405 & 15991 & 290948 & $5.20 \mathrm{E}-02$ & 125905 & 18.27 & 559 & 5.07E-02 & $2.56 \mathrm{E}-04$ & $1.21 \mathrm{E}-05$ & $2.81 \mathrm{E}-04$ & 4.46 & 1.9953 & 1.03 \\
\hline 0 & 45631 & 16129 & 15716 & 307060 & $5.08 \mathrm{E}-02$ & 129751 & 18.82 & 562 & $4.98 \mathrm{E}-02$ & $1.25 \mathrm{E}-04$ & $1.58 \mathrm{E}-05$ & $1.56 \mathrm{E}-04$ & 4.46 & 1.9952 & 1.02 \\
\hline 0 & 45975 & 16267 & 15578 & 305733 & $5.13 \mathrm{E}-02$ & 130364 & 18.91 & 564 & $4.93 \mathrm{E}-02$ & $1.00 \mathrm{E}-04$ & $1.11 \mathrm{E}-05$ & $1.22 \mathrm{E}-04$ & 4.46 & 1.9951 & 1.04 \\
\hline 0 & 43149 & 15578 & 15302 & 280414 & 4.97E-02 & 115792 & 16.80 & 564 & 4.85E-02 & 4.37E- 05 & $3.26 \mathrm{E}-06$ & $5.02 E-05$ & 4.46 & 1.9951 & 1.02 \\
\hline 0 & 47147 & 18266 & 17990 & 322703 & $6.07 \mathrm{E}-02$ & 162985 & 23.65 & 563 & $6.05 \mathrm{E}-02$ & $2.75 \mathrm{E}-05$ & 2.55E-06 & $3.26 \mathrm{E}-05$ & 4.46 & 1.9951 & 1.00 \\
\hline 0 & 38876 & 15164 & 15233 & 310686 & 5.07E- 02 & 130834 & 18.98 & 561 & $5.12 \mathrm{E}-02$ & 3.77E-05 & 2.79E-06 & 4.32E-05 & 4.46 & 1.9951 & 0.99 \\
\hline 0 & 38876 & 14958 & 15302 & 335728 & 4.95E-02 & 138246 & 20.06 & 562 & 5.14E-02 & $1.55 \mathrm{E}-05$ & $1.18 \mathrm{E}-06$ & $1.79 \mathrm{E}-05$ & 4.46 & 1.9951 & 0.96 \\
\hline 0 & 38738 & 14889 & 15233 & 337808 & 4.93E-02 & 138369 & 20.07 & 561 & $5.12 \mathrm{E}-02$ & $2.48 \mathrm{E}-05$ & 2.44E-06 & 2.97E-05 & 4.46 & 1.9951 & 0.96 \\
\hline 0 & 38807 & 14958 & 15164 & 332313 & 4.96E-02 & 136992 & 19.87 & 561 & $5.10 \mathrm{E}-02$ & 3.37E-05 & 1.99E-06 & 3.77E-05 & 4.46 & 1.9951 & 0.97 \\
\hline 0 & 39220 & 15647 & 14889 & 275580 & $5.30 \mathrm{E}-02$ & 121511 & 17.63 & 560 & $5.00 \mathrm{E}-02$ & $2.76 \mathrm{E}-05$ & $1.13 \mathrm{E}-06$ & 2.99E-05 & 4.46 & 1.9950 & 1.06 \\
\hline 0 & 38393 & 15647 & 14751 & 247677 & $5.38 \mathrm{E}-02$ & 110792 & 16.07 & 554 & 4.96E-02 & 3.72E-05 & 2.47E-06 & 4.21E-05 & 4.46 & 1.9950 & 1.09 \\
\hline 0 & 39910 & 15991 & 15233 & 269485 & $5.44 \mathrm{E}-02$ & 121795 & 17.67 & 554 & $5.12 \mathrm{E}-02$ & $4.33 \mathrm{E}-05$ & 3.49E-06 & $5.03 \mathrm{E}-05$ & 4.46 & 1.9950 & 1.06 \\
\hline 0 & 39496 & 15854 & 15164 & 267088 & $5.40 \mathrm{E}-02$ & 119812 & 17.38 & 552 & $5.10 \mathrm{E}-02$ & $3.26 \mathrm{E}-05$ & $1.24 \mathrm{E}-06$ & $3.51 \mathrm{E}-05$ & 4.46 & 1.9950 & 1.06 \\
\hline 0 & 39979 & 16060 & 15371 & 265986 & $5.47 \mathrm{E}-02$ & 120940 & 17.55 & 552 & $5.17 \mathrm{E}-02$ & $3.36 \mathrm{E}-05$ & $1.77 \mathrm{E}-06$ & $3.71 \mathrm{E}-05$ & 4.46 & 1.9950 & 1.06 \\
\hline
\end{tabular}




\begin{tabular}{|c|c|c|c|c|c|c|}
\hline \multicolumn{7}{|c|}{ RGA Data } \\
\hline $\begin{array}{l}\text { Total } \\
\text { press }\end{array}$ & Ch 1 & Ch 2 & Ch 3 & Ch 4 & Ch 12 & $\begin{array}{c}\text { Ion } \\
\text { pressure }\end{array}$ \\
\hline AMU/chg & 4 & 28 & 40 & 44 & 14 & torr \\
\hline $8.70 \mathrm{E}-06$ & $1.0 \mathrm{E}-07$ & $1.7 \mathrm{E}-07$ & $4.9 \mathrm{E}-06$ & $8.4 \mathrm{E}-10$ & 6.7E-10 & $6.90 \mathrm{E}-06$ \\
\hline $8.50 \mathrm{E}-06$ & 1.7E- 08 & 1.4E- 08 & $6.2 \mathrm{E}-06$ & $1.3 \mathrm{E}-09$ & $2.9 \mathrm{E}-10$ & 7.40E-06 \\
\hline $1.00 \mathrm{E}-05$ & $1.4 \mathrm{E}-08$ & $9.9 \mathrm{E}-09$ & $5.9 \mathrm{E}-06$ & $1.3 \mathrm{E}-09$ & $2.9 \mathrm{E}-10$ & $8.00 \mathrm{E}-06$ \\
\hline $1.00 \mathrm{E}-05$ & $5.6 \mathrm{E}-08$ & $1.1 \mathrm{E}-08$ & $8.6 \mathrm{E}-06$ & $1.6 \mathrm{E}-09$ & $1.9 \mathrm{E}-10$ & $8.00 \mathrm{E}-06$ \\
\hline $1.10 \mathrm{E}-05$ & $5.3 \mathrm{E}-08$ & $9.3 \mathrm{E}-09$ & $3.3 \mathrm{E}-06$ & $1.0 \mathrm{E}-09$ & $2.9 \mathrm{E}-10$ & $8.30 \mathrm{E}-06$ \\
\hline $1.00 \mathrm{E}-05$ & $5.6 \mathrm{E}-08$ & $8.9 \mathrm{E}-09$ & 5.5E-06 & $1.2 \mathrm{E}-09$ & $2.4 \mathrm{E}-10$ & $8.10 \mathrm{E}-06$ \\
\hline $9.90 \mathrm{E}-06$ & 4.6E- 08 & BE-09 & $9.9 \mathrm{E}-06$ & $1 \mathrm{E}-09$ & $2.4 \mathrm{E}-10$ & 10E-06 \\
\hline $1.00 \mathrm{E}-05$ & $5.4 \mathrm{E}-08$ & AE-09 & $1 \mathrm{E}-05$ & $7 \mathrm{E}-09$ & $1.4 \mathrm{E}-10$ & $8.20 \mathrm{E}-06$ \\
\hline $1.00 \mathrm{E}-05$ & 4.5E-08 & 7.7E-09 & $1.0 \mathrm{E}-05$ & 5.7E-10 & $2.4 \mathrm{E}-10$ & $8.10 \mathrm{E}-06$ \\
\hline $9.90 \mathrm{E}-06$ & $6.3 \mathrm{E}-08$ & $7.8 \mathrm{E}-09$ & $6.7 \mathrm{E}-06$ & $9.5 \mathrm{E}-10$ & $9.5 \mathrm{E}-11$ & $8.00 \mathrm{E}-06$ \\
\hline $9.80 \mathrm{E}-06$ & 5.9E-08 & $8.4 \mathrm{E}-09$ & $7.2 \mathrm{E}-06$ & $1.1 \mathrm{E}-09$ & $1.4 \mathrm{E}-10$ & $8.40 \mathrm{E}-06$ \\
\hline $1.00 \mathrm{E}-05$ & 5.7E-08 & $8.4 \mathrm{E}-09$ & $7.4 \mathrm{E}-06$ & $9.2 \mathrm{E}-10$ & $1.4 \mathrm{E}-10$ & $8.30 \mathrm{E}-06$ \\
\hline $1.10 \mathrm{E}-05$ & $6.9 \mathrm{E}-08$ & $9.3 \mathrm{E}-09$ & $8.0 \mathrm{E}-06$ & $9.2 \mathrm{E}-10$ & $1.9 \mathrm{E}-10$ & $8.60 \mathrm{E}-06$ \\
\hline $1.50 \mathrm{E}-05$ & $5.3 \mathrm{E}-07$ & $1.4 \mathrm{E}-06$ & 5.9E-06 & 2.3E-09 & $4.5 \mathrm{E}-09$ & $7.70 \mathrm{E}-06$ \\
\hline $1.30 \mathrm{E}-05$ & 3.1E-07 & $6.6 \mathrm{E}-08$ & $8.2 \mathrm{E}-06$ & 3.1E-09 & $1.0 \mathrm{E}-09$ & $8.20 \mathrm{E}-06$ \\
\hline $1.30 \mathrm{E}-05$ & $3.8 \mathrm{E}-07$ & $3.3 \mathrm{E}-08$ & $8.9 \mathrm{E}-06$ & 4.1E-09 & $1.0 \mathrm{E}-09$ & $8.80 \mathrm{E}-06$ \\
\hline $1.40 \mathrm{E}-05$ & 3.3E-07 & $2.8 \mathrm{E}-08$ & $8.9 \mathrm{E}-06$ & 3.1E-09 & $9.0 \mathrm{E}-10$ & $9.40 \mathrm{E}-06$ \\
\hline $1.70 \mathrm{E}-05$ & 2.7E-07 & $1.5 \mathrm{E}-08$ & $6.8 \mathrm{E}-06$ & $1.1 \mathrm{E}-09$ & $3.8 \mathrm{E}-10$ & $8.80 \mathrm{E}-06$ \\
\hline $2.20 \mathrm{E}-05$ & $2.4 \mathrm{E}-07$ & $1.0 \mathrm{E}-08$ & $7.2 \mathrm{E}-06$ & $9.3 \mathrm{E}-10$ & $2.4 \mathrm{E}-10$ & $1.50 \mathrm{E}-05$ \\
\hline $2.20 \mathrm{E}-05$ & 4.0E- 07 & $1.6 \mathrm{E}-08$ & $1.0 \mathrm{E}-05$ & $1.2 \mathrm{E}-09$ & $2.9 \mathrm{E}-10$ & $1.30 \mathrm{E}-05$ \\
\hline $3.00 \mathrm{E}-05$ & 3.5E-07 & $9.1 \mathrm{E}-09$ & $8.3 \mathrm{E}-06$ & $6.9 \mathrm{E}-10$ & $1.9 \mathrm{E}-10$ & $1.30 \mathrm{E}-05$ \\
\hline $2.40 \mathrm{E}-05$ & $4.8 \mathrm{E}-07$ & $1.2 \mathrm{E}-08$ & $9.1 \mathrm{E}-06$ & $1.1 \mathrm{E}-09$ & $2.9 \mathrm{E}-10$ & $1.30 \mathrm{E}-05$ \\
\hline $1.90 \mathrm{E}-05$ & 3.3E-07 & $1.3 \mathrm{E}-08$ & $1.0 \mathrm{E}-05$ & 7.4E-10 & $2.9 \mathrm{E}-10$ & $1.30 \mathrm{E}-05$ \\
\hline $2.10 \mathrm{E}-05$ & 3.0E-07 & $1.2 \mathrm{E}-08$ & $8.8 \mathrm{E}-06$ & 4.8E-10 & $1.9 \mathrm{E}-10$ & $1.40 \mathrm{E}-05$ \\
\hline $2.20 \mathrm{E}-05$ & 4.4E- 07 & 1.6E-08 & $1.2 \mathrm{E}-05$ & $1.1 \mathrm{E}-09$ & 2.9E-10 & $1.40 \mathrm{E}-05$ \\
\hline $1.80 \mathrm{E}-05$ & $5.6 \mathrm{E}-07$ & $1.5 \mathrm{E}-08$ & $1.2 \mathrm{E}-05$ & $1.2 \mathrm{E}-09$ & $2.9 \mathrm{E}-10$ & 1.30E-05 \\
\hline 2.40E-05 & 4.0E-07 & $1.5 \mathrm{E}-08$ & 1.1E-05 & $5.9 \mathrm{E}-10$ & 3.3E-10 & $1.20 \mathrm{E}-05$ \\
\hline $2.00 \mathrm{E}-05$ & $2.5 \mathrm{E}-07$ & $1.3 \mathrm{E}-08$ & $8.3 \mathrm{E}-06$ & $6.9 \mathrm{E}-10$ & $3.3 \mathrm{E}-10$ & $1.20 \mathrm{E}-05$ \\
\hline
\end{tabular}




\section{Appendix G \\ MRM Can L1303 Reduction Calculation using the Calibration Gas Sum of the RGA Partial Pressure}




\begin{tabular}{|c|c|c|c|c|c|c|c|c|c|c|c|c|c|}
\hline Method & \multicolumn{2}{|c|}{ Psum Pressure } & \multicolumn{6}{|c|}{ Data from executed procedures } & \multicolumn{5}{|c|}{ Partial Pressure calculations } \\
\hline \multirow{2}{*}{ Date } & \multirow{2}{*}{$\begin{array}{l}\text { Manifold } \\
\text { / Station }\end{array}$} & \multirow{2}{*}{ Can } & $\begin{array}{l}\text { Manifold } \\
\text { pressure }\end{array}$ & $\begin{array}{l}\text { Man Prel } \\
A, B \text { or } C\end{array}$ & $\begin{array}{l}\text { Man rel } \\
\mathrm{A}, \mathrm{B} \text { or } \mathrm{C}\end{array}$ & $\begin{array}{l}\text { Release } \\
\text { pres }\end{array}$ & Calc Pr & $\mathrm{CO} \mathrm{Pr}$ & $\begin{array}{l}\text { Calc CO } \\
\text { (sum PP) }\end{array}$ & $\begin{array}{l}\text { Calc He } \\
\text { (sum PP) }\end{array}$ & $\begin{array}{l}\text { Calc CO2 } \\
\text { (sum PP) }\end{array}$ & $\begin{array}{l}\mathrm{CO}+\mathrm{He} \\
\text { (sum PP) }\end{array}$ & $\begin{array}{l}\text { Tmax } \\
\text { (sum PP) }\end{array}$ \\
\hline & & & psia & psia & psia & psia & psia & $\mathrm{Pa}$ & $\mathrm{Pa}$ & $\mathrm{Pa}$ & $\mathrm{Pa}$ & psia & $\mathrm{K}$ \\
\hline $5 / 31 / 2017$ & $\mathrm{~A} / 3$ & L1303 & 21.17 & & 7.42 & 7.45 & 21.16 & 2896 & 4828 & 2832 & 24 & 1.11 & 1097 \\
\hline $6 / 8 / 2017$ & $\mathrm{~A} / 3$ & L1303 & 20.01 & & 7.02 & 7.00 & 20.01 & 222 & 304 & 371 & 29 & 0.10 & 841 \\
\hline $6 / 14 / 2017$ & $A / 3$ & L1303 & 19.96 & & 7.03 & 6.92 & 19.98 & 136 & 228 & 323 & 31 & 0.08 & 819 \\
\hline $6 / 21 / 2017$ & $A / 3$ & L1303 & & 6.78 & 2.42 & 2.36 & 20.04 & 156 & 180 & 903 & 25 & 0.16 & 810 \\
\hline $6 / 28 / 2017$ & $\mathrm{~A} / 3$ & L1303 & & 6.78 & 2.43 & 2.34 & 20.02 & 117 & 389 & 2220 & 44 & 0.38 & 843 \\
\hline $7 / 6 / 2017$ & $\mathrm{~A} / 3$ & $\mathrm{~L} 1303$ & & 6.78 & 2.42 & 2.37 & 20.01 & 123 & 222 & 1385 & 31 & 0.23 & 817 \\
\hline $7 / 12 / 2017$ & $A / 3$ & L1303 & & 6.79 & 2.43 & 2.38 & 20.05 & 116 & 115 & 639 & 16 & 0.11 & 797 \\
\hline $7 / 19 / 2017$ & $A / 3$ & L1303 & & 6.76 & 2.42 & 2.34 & 20.01 & 130 & 115 & 660 & 21 & 0.11 & 788 \\
\hline $7 / 25 / 2017$ & $\mathrm{~A} / 3$ & L1303 & & 6.76 & 2.42 & 2.37 & 20.06 & 107 & 102 & 591 & 8 & 0.10 & 812 \\
\hline $8 / 2 / 2017$ & $A / 3$ & L1303 & & 6.78 & 2.43 & 2.38 & 20.02 & 109 & 157 & 1272 & 19 & 0.21 & 810 \\
\hline $8 / 9 / 2017$ & $A / 3$ & L1303 & & 6.75 & 2.42 & 2.33 & 20.02 & 118 & 159 & 1111 & 22 & 0.18 & 807 \\
\hline $8 / 16 / 2017$ & $A / 3$ & L1303 & & 6.78 & 2.42 & 2.36 & 20.05 & 116 & 155 & 1059 & 17 & 0.18 & 813 \\
\hline $8 / 24 / 2017$ & $A / 3$ & L1303 & & 6.48 & 2.32 & 2.26 & 20.00 & 116 & 158 & 1163 & 16 & 0.19 & 817 \\
\hline $11 / 6 / 2017$ & $\mathrm{~A} / 3$ & L1303 & & 8.32 & 2.95 & 2.89 & 20.04 & 12952 & 24732 & 9244 & 40 & 4.93 & 1285 \\
\hline $12 / 6 / 2017$ & $\mathrm{~A} / 3$ & L1303 & & 6.65 & 2.38 & 2.32 & 20.07 & 701 & 1064 & 5044 & 50 & 0.89 & 913 \\
\hline $1 / 10 / 2018$ & $A / 3$ & L1303 & & 6.62 & 2.34 & 2.28 & 20.05 & 347 & 484 & 5714 & 61 & 0.90 & 846 \\
\hline $2 / 7 / 2018$ & $\mathrm{~A} / 3$ & L1303 & & 6.67 & 2.36 & 2.26 & 20.03 & 281 & 425 & 4984 & 47 & 0.78 & 847 \\
\hline $3 / 5 / 2018$ & $A / 3$ & L1303 & & 6.26 & 2.26 & 2.22 & 20.00 & 124 & 299 & 5361 & 22 & 0.82 & 848 \\
\hline $4 / 4 / 2018$ & $C / 27$ & L1303 & & 6.84 & 2.65 & 2.61 & 20.09 & 63 & 187 & 4567 & 17 & 0.69 & 825 \\
\hline $5 / 2 / 2018$ & $\mathrm{C} / 27$ & L1303 & & 5.64 & 2.20 & 2.21 & 20.01 & 102 & 215 & 5344 & 16 & 0.81 & 837 \\
\hline $6 / 6 / 2018$ & $C / 27$ & L1303 & & 5.64 & 2.17 & 2.22 & 20.02 & 42 & 144 & 5516 & 11 & 0.82 & 823 \\
\hline $7 / 11 / 2018$ & $C / 27$ & L1303 & & 5.62 & 2.16 & 2.21 & 20.00 & 67 & 166 & 6802 & 16 & 1.01 & 819 \\
\hline $8 / 7 / 2018$ & $\mathrm{C} / 27$ & L1303 & & 5.63 & 2.17 & 2.20 & 20.00 & 91 & 163 & 4255 & 10 & 0.64 & 835 \\
\hline $9 / 6 / 2018$ & $\mathrm{C} / 27$ & L1303 & & 5.69 & 2.27 & 2.16 & 20.07 & 76 & 176 & 4573 & 7 & 0.69 & 850 \\
\hline $10 / 4 / 2018$ & $\mathrm{C} / 27$ & L1303 & & 5.57 & 2.27 & 2.14 & 19.98 & 103 & 189 & 5073 & 13 & 0.76 & 836 \\
\hline $11 / 12 / 2018$ & $\mathrm{C} / 27$ & L1303 & & 5.79 & 2.32 & 2.21 & 20.06 & 117 & 169 & 6230 & 14 & 0.93 & 826 \\
\hline $12 / 12 / 2018$ & $\mathrm{C} / 27$ & L1303 & & 5.73 & 2.30 & 2.20 & 20.06 & 88 & 192 & 5071 & 7 & 0.76 & 856 \\
\hline $1 / 9 / 2019$ & $\mathrm{C} / 27$ & L1303 & & 5.80 & 2.33 & 2.23 & 20.06 & 90 & 210 & 3994 & 11 & 0.61 & 848 \\
\hline
\end{tabular}




\begin{tabular}{|c|c|c|c|c|c|c|c|c|c|c|c|c|c|c|c|c|}
\hline \multicolumn{3}{|c|}{ Release Pressures } & \multirow[b]{2}{*}{$\begin{array}{c}\text { Release } \\
\text { pres }\end{array}$} & \multicolumn{4}{|c|}{ Can pressure - double rel. } & \multirow[b]{2}{*}{$\begin{array}{c}\text { Can } \\
\text { Temp }\end{array}$} & \multicolumn{8}{|c|}{ Reduction Calculation } \\
\hline $\begin{array}{l}\text { Manifold } \\
\text { pressure }\end{array}$ & \begin{tabular}{|c|} 
Man rel \\
A,BorC \\
\end{tabular} & $\begin{array}{c}\text { Man rel } \\
\text { A,BorC }\end{array}$ & & $\begin{array}{c}\text { Variable } \\
(\mathrm{T} / \mathrm{V})\end{array}$ & $\begin{array}{l}\text { N (MRM } \\
\text { can) }\end{array}$ & $\mathrm{P}$ (calc) & $\mathrm{P}($ calc $)$ & & $\begin{array}{c}\# \text { mol } \\
\text { gas }\end{array}$ & $\begin{array}{c}\text { \# mol } \\
\text { gas }\end{array}$ & $\begin{array}{c}\% \text { in } \\
\text { can } \\
\end{array}$ & $\# \mathrm{~mol} \mathrm{CO}$ & $\begin{array}{c}\mathrm{mol} \\
\mathrm{CO} 2 \\
\end{array}$ & $\begin{array}{c}\text { \#mol of } \\
\text { "O" }\end{array}$ & \multirow{2}{*}{$\begin{array}{c}\mathrm{O} 2 \\
\text { portion } \\
\text { PuO2 } \\
\mathrm{mol} \\
\end{array}$} & \multirow[t]{2}{*}{ PuOx } \\
\hline $\mathrm{Pa}$ & $\mathrm{Pa}$ & $\mathrm{Pa}$ & $\mathrm{Pa}$ & & & $\mathrm{Pa}$ & psia & $\mathrm{K}$ & $\mathrm{mol}$ & $\mathrm{mol}$ & ratio & $\mathrm{mol}$ & $\mathrm{mol}$ & mol & & \\
\hline 145922 & 0 & 51145 & 51352 & & & & & 558 & $1.65 \mathrm{E}-01$ & 5.96E-02 & $36 \%$ & $5.76 \mathrm{E}-03$ & $2.81 \mathrm{E}-05$ & 5.82E-03 & 4.47 & 1.9974 \\
\hline 137926 & 0 & 48388 & 48250 & & & & & 563 & $1.56 \mathrm{E}-01$ & 5.63E-02 & $36 \%$ & 3.45E-04 & $3.25 \mathrm{E}-05$ & $4.10 \mathrm{E}-04$ & 4.47 & 1.9972 \\
\hline 137581 & 0 & 48457 & 47699 & & & & & 561 & $1.56 \mathrm{E}-01$ & $5.62 \mathrm{E}-02$ & $36 \%$ & $2.58 \mathrm{E}-04$ & 3.49E-05 & $3.28 \mathrm{E}-04$ & 4.47 & 1.9971 \\
\hline 0 & 46734 & 16681 & 16267 & 294329 & $5.28 \mathrm{E}-02$ & 129315 & 18.76 & 559 & $5.15 E-02$ & $6.64 \mathrm{E}-03$ & $13 \%$ & $6.78 \mathrm{E}-05$ & $9.45 \mathrm{E}-06$ & 8.67E-05 & 4.47 & 1.9970 \\
\hline 0 & 46734 & 16750 & 16129 & 289176 & $5.32 \mathrm{E}-02$ & 127869 & 18.55 & 558 & $5.11 \mathrm{E}-02$ & $6.59 \mathrm{E}-03$ & $13 \%$ & 1.47E-04 & $1.65 \mathrm{E}-05$ & 1.80E-04 & 4.47 & 1.9969 \\
\hline 0 & 46734 & 16681 & 16336 & 294329 & $5.28 \mathrm{E}-02$ & 129315 & 18.76 & 558 & 5.17E-02 & 6.67E-03 & $13 \%$ & $8.41 \mathrm{E}-05$ & 1.17E-05 & 1.07E-04 & 4.47 & 1.9969 \\
\hline 0 & 46803 & 16750 & 16405 & 291002 & $5.31 \mathrm{E}-02$ & 128570 & 18.65 & 558 & $5.20 \mathrm{E}-02$ & $6.70 \mathrm{E}-03$ & $13 \%$ & 4.35E-05 & $6.00 \mathrm{E}-06$ & 5.55E-05 & 4.47 & 1.9969 \\
\hline 0 & 46596 & 16681 & 16129 & 290623 & $5.29 \mathrm{E}-02$ & 127896 & 18.55 & 560 & $5.11 \mathrm{E}-02$ & $6.59 \mathrm{E}-03$ & $13 \%$ & $4.28 \mathrm{E}-05$ & $7.81 \mathrm{E}-06$ & $5.84 \mathrm{E}-05$ & 4.46 & 1.9969 \\
\hline 0 & 46596 & 16681 & 16336 & 290623 & $5.29 \mathrm{E}-02$ & 127896 & 18.55 & 559 & 5.17E-02 & 6.67E-03 & $13 \%$ & $3.84 \mathrm{E}-05$ & 2.84E-06 & 4.41E-05 & 4.46 & 1.9968 \\
\hline 0 & 46734 & 16750 & 16405 & 289176 & $5.32 \mathrm{E}-02$ & 127869 & 18.55 & 559 & $5.20 \mathrm{E}-02$ & $6.70 \mathrm{E}-03$ & $13 \%$ & 5.99E-05 & 7.33E-06 & 7.46E-05 & 4.46 & 1.9968 \\
\hline 0 & 46527 & 16681 & 16060 & 288793 & $5.30 \mathrm{E}-02$ & 127196 & 18.45 & 558 & 5.09E-02 & $6.56 \mathrm{E}-03$ & $13 \%$ & 5.93E-05 & 8.07E-06 & $7.54 \mathrm{E}-05$ & 4.46 & 1.9968 \\
\hline 0 & 46734 & 16681 & 16267 & 294329 & $5.28 \mathrm{E}-02$ & 129315 & 18.76 & 559 & $5.15 \mathrm{E}-02$ & $6.64 \mathrm{E}-03$ & $13 \%$ & 5.83E-05 & $6.44 \mathrm{E}-06$ & $7.12 \mathrm{E}-05$ & 4.46 & 1.9967 \\
\hline 0 & 44666 & 15991 & 15578 & 290497 & 5.07E-02 & 122565 & 17.78 & 559 & 4.93E-02 & $6.36 \mathrm{E}-03$ & $13 \%$ & 5.64E-05 & $5.66 \mathrm{E}-06$ & $6.77 \mathrm{E}-05$ & 4.46 & 1.9967 \\
\hline 0 & 57349 & 20334 & 19920 & 302934 & $6.42 \mathrm{E}-02$ & 161650 & 23.45 & 558 & $6.31 \mathrm{E}-02$ & $8.13 \mathrm{E}-03$ & $13 \%$ & $1.13 \mathrm{E}-02$ & $2.42 \mathrm{E}-05$ & 1.13E-02 & 4.45 & 1.9916 \\
\hline 0 & 45838 & 16405 & 15991 & 290948 & $5.20 \mathrm{E}-02$ & 125905 & 18.27 & 559 & 5.07E-02 & $6.53 \mathrm{E}-03$ & $13 \%$ & $3.90 \mathrm{E}-04$ & $1.92 \mathrm{E}-05$ & $4.28 \mathrm{E}-04$ & 4.45 & 1.9914 \\
\hline 0 & 45631 & 16129 & 15716 & 307060 & $5.08 \mathrm{E}-02$ & 129751 & 18.82 & 562 & $4.98 \mathrm{E}-02$ & $6.42 \mathrm{E}-03$ & $13 \%$ & $1.74 \mathrm{E}-04$ & $2.31 \mathrm{E}-05$ & 2.21E-04 & 4.45 & 1.9913 \\
\hline 0 & 45975 & 16267 & 15578 & 305733 & 5.13E-02 & 130364 & 18.91 & 564 & 4.93E-02 & $6.36 \mathrm{E}-03$ & $13 \%$ & $1.52 \mathrm{E}-04$ & $1.75 \mathrm{E}-05$ & 1.87E-04 & 4.45 & 1.9913 \\
\hline 0 & 43149 & 15578 & 15302 & 280414 & 4.97E-02 & 115792 & 16.80 & 564 & $4.85 E-02$ & $6.25 \mathrm{E}-03$ & $13 \%$ & $1.05 E-04$ & 8.17E-06 & $1.21 \mathrm{E}-04$ & 4.45 & 1.9912 \\
\hline 0 & 47147 & 18266 & 17990 & 322703 & 6.07E-02 & 162985 & 23.65 & 563 & $6.05 \mathrm{E}-02$ & $7.35 \mathrm{E}-03$ & $12 \%$ & $8.18 \mathrm{E}-05$ & 7.85E-06 & $9.75 \mathrm{E}-05$ & 4.45 & 1.9912 \\
\hline 0 & 38876 & 15164 & 15233 & 310686 & 5.07E- 02 & 130834 & 18.98 & 561 & $5.12 \mathrm{E}-02$ & $6.22 \mathrm{E}-03$ & $12 \%$ & 7.98E-05 & $6.16 \mathrm{E}-06$ & $9.22 \mathrm{E}-05$ & 4.45 & 1.9911 \\
\hline 0 & 38876 & 14958 & 15302 & 335728 & 4.95E-02 & 138246 & 20.06 & 562 & $5.14 \mathrm{E}-02$ & $6.25 \mathrm{E}-03$ & $12 \%$ & 5.37E-05 & 4.24E-06 & $6.22 \mathrm{E}-05$ & 4.45 & 1.9911 \\
\hline 0 & 38738 & 14889 & 15233 & 337808 & 4.93E-02 & 138369 & 20.07 & 561 & $5.12 \mathrm{E}-02$ & $6.22 \mathrm{E}-03$ & $12 \%$ & 6.17E-05 & $6.39 \mathrm{E}-06$ & 7.45E-05 & 4.45 & 1.9911 \\
\hline 0 & 38807 & 14958 & 15164 & 332313 & 4.96E-02 & 136992 & 19.87 & 561 & $5.10 \mathrm{E}-02$ & $6.19 \mathrm{E}-03$ & $12 \%$ & $6.01 \mathrm{E}-05$ & 3.67E-06 & $6.74 \mathrm{E}-05$ & 4.45 & 1.9910 \\
\hline 0 & 39220 & 15647 & 14889 & 275580 & $5.30 \mathrm{E}-02$ & 121511 & 17.63 & 560 & $5.00 \mathrm{E}-02$ & $6.08 \mathrm{E}-03$ & $12 \%$ & $6.36 \mathrm{E}-05$ & $2.70 \mathrm{E}-06$ & $6.90 \mathrm{E}-05$ & 4.45 & 1.9910 \\
\hline 0 & 38393 & 15647 & 14751 & 247677 & $5.38 \mathrm{E}-02$ & 110792 & 16.07 & 554 & 4.96E-02 & $6.02 \mathrm{E}-03$ & $12 \%$ & $6.80 \mathrm{E}-05$ & 4.69E-06 & 7.73E-05 & 4.45 & 1.9910 \\
\hline 0 & 39910 & 15991 & 15233 & 269485 & $5.44 \mathrm{E}-02$ & 121795 & 17.67 & 554 & $5.12 \mathrm{E}-02$ & $6.22 \mathrm{E}-03$ & $12 \%$ & $6.26 \mathrm{E}-05$ & 5.29E-06 & $7.32 \mathrm{E}-05$ & 4.45 & 1.9909 \\
\hline 0 & 39496 & 15854 & 15164 & 267088 & $5.40 \mathrm{E}-02$ & 119812 & 17.38 & 552 & 5.10E-02 & $6.19 \mathrm{E}-03$ & $12 \%$ & $7.08 \mathrm{E}-05$ & $2.81 \mathrm{E}-06$ & 7.64E-05 & 4.45 & 1.9909 \\
\hline 0 & 39979 & 16060 & 15371 & 265986 & 5.47E-02 & 120940 & 17.55 & 552 & 5.17E-02 & $6.28 \mathrm{E}-03$ & $12 \%$ & 7.86E-05 & 4.28E-06 & $8.71 \mathrm{E}-05$ & 4.45 & 1.9909 \\
\hline
\end{tabular}




\begin{tabular}{|c|c|c|c|c|c|c|c|c|}
\hline \multirow[b]{2}{*}{$\begin{array}{l}\text { Ratio of } \\
\text { mol calc }\end{array}$} & \multicolumn{8}{|c|}{ RGA Data } \\
\hline & $\mathrm{P}$ (sum PP) & Total press & Ch 1 & Ch 2 & Ch 3 & Ch 4 & Ch 12 & Ion pressure \\
\hline & torr & AMU/chg & 4 & 28 & 40 & 44 & 14 & \\
\hline & $5.22 \mathrm{E}-06$ & $8.70 \mathrm{E}-06$ & $1.01 \mathrm{E}-07$ & $1.73 \mathrm{E}-07$ & $4.95 \mathrm{E}-06$ & $8.43 \mathrm{E}-10$ & $6.67 \mathrm{E}-10$ & $6.90 \mathrm{E}-06$ \\
\hline & $6.20 \mathrm{E}-06$ & $8.50 \mathrm{E}-06$ & $1.67 \mathrm{E}-08$ & $1.37 \mathrm{E}-08$ & $6.17 \mathrm{E}-06$ & $1.29 \mathrm{E}-09$ & $2.86 \mathrm{E}-10$ & $7.40 \mathrm{E}-06$ \\
\hline & $5.97 \mathrm{E}-06$ & $1.00 \mathrm{E}-05$ & $1.40 \mathrm{E}-08$ & $9.85 \mathrm{E}-09$ & $5.94 \mathrm{E}-06$ & $1.33 \mathrm{E}-09$ & $2.86 \mathrm{E}-10$ & 8.00E-06 \\
\hline 1.03 & $8.63 \mathrm{E}-06$ & $1.00 \mathrm{E}-05$ & $5.64 \mathrm{E}-08$ & $1.13 \mathrm{E}-08$ & $8.56 \mathrm{E}-06$ & $1.57 \mathrm{E}-09$ & $1.90 \mathrm{E}-10$ & $8.00 \mathrm{E}-06$ \\
\hline 1.04 & $3.31 \mathrm{E}-06$ & $1.10 \mathrm{E}-05$ & $5.33 \mathrm{E}-08$ & $9.33 \mathrm{E}-09$ & $3.25 \mathrm{E}-06$ & $1.05 \mathrm{E}-09$ & $2.86 \mathrm{E}-10$ & $8.30 \mathrm{E}-06$ \\
\hline 1.02 & 5.55E-06 & $1.00 \mathrm{E}-05$ & $5.57 \mathrm{E}-08$ & 8.91E-09 & $5.48 \mathrm{E}-06$ & $1.24 \mathrm{E}-09$ & $2.38 \mathrm{E}-10$ & $8.10 \mathrm{E}-06$ \\
\hline 1.02 & $9.94 \mathrm{E}-06$ & $9.90 \mathrm{E}-06$ & $4.60 \mathrm{E}-08$ & $8.28 \mathrm{E}-09$ & $9.88 \mathrm{E}-06$ & $1.14 \mathrm{E}-09$ & $2.38 \mathrm{E}-10$ & $8.10 \mathrm{E}-06$ \\
\hline 1.04 & $1.13 \mathrm{E}-05$ & $1.00 \mathrm{E}-05$ & $5.40 \mathrm{E}-08$ & $9.40 \mathrm{E}-09$ & $1.12 \mathrm{E}-05$ & $1.71 \mathrm{E}-09$ & $1.43 \mathrm{E}-10$ & $8.20 \mathrm{E}-06$ \\
\hline 1.02 & $1.04 \mathrm{E}-05$ & $1.00 \mathrm{E}-05$ & 4.47E-08 & 7.71E-09 & $1.04 \mathrm{E}-05$ & $5.71 \mathrm{E}-10$ & $2.38 \mathrm{E}-10$ & $8.10 \mathrm{E}-06$ \\
\hline 1.02 & $6.82 \mathrm{E}-06$ & $9.90 \mathrm{E}-06$ & $6.28 \mathrm{E}-08$ & $7.78 \mathrm{E}-09$ & $6.75 \mathrm{E}-06$ & $9.52 \mathrm{E}-10$ & $9.52 \mathrm{E}-11$ & $8.00 \mathrm{E}-06$ \\
\hline 1.04 & 7.27E-06 & $9.80 \mathrm{E}-06$ & $5.86 \mathrm{E}-08$ & 8.39E-09 & $7.20 \mathrm{E}-06$ & $1.14 \mathrm{E}-09$ & $1.43 \mathrm{E}-10$ & $8.40 \mathrm{E}-06$ \\
\hline 1.03 & $7.45 \mathrm{E}-06$ & $1.00 \mathrm{E}-05$ & $5.71 \mathrm{E}-08$ & 8.36E-09 & 7.39E-06 & $9.24 \mathrm{E}-10$ & $1.43 \mathrm{E}-10$ & 8.30E-06 \\
\hline 1.03 & $8.12 \mathrm{E}-06$ & $1.10 \mathrm{E}-05$ & $6.86 \mathrm{E}-08$ & $9.28 \mathrm{E}-09$ & $8.05 \mathrm{E}-06$ & $9.24 \mathrm{E}-10$ & $1.90 \mathrm{E}-10$ & $8.60 \mathrm{E}-06$ \\
\hline 1.02 & $7.85 \mathrm{E}-06$ & $1.50 \mathrm{E}-05$ & $5.26 \mathrm{E}-07$ & $1.41 \mathrm{E}-06$ & $5.92 \mathrm{E}-06$ & $2.27 \mathrm{E}-09$ & $4.48 \mathrm{E}-09$ & 7.70E-06 \\
\hline 1.03 & $8.55 \mathrm{E}-06$ & $1.30 \mathrm{E}-05$ & $3.12 \mathrm{E}-07$ & $6.58 \mathrm{E}-08$ & 8.17E-06 & $3.09 \mathrm{E}-09$ & $1.00 \mathrm{E}-09$ & $8.20 \mathrm{E}-06$ \\
\hline 1.02 & $9.31 \mathrm{E}-06$ & $1.30 \mathrm{E}-05$ & $3.85 \mathrm{E}-07$ & $3.26 \mathrm{E}-08$ & $8.89 \mathrm{E}-06$ & 4.13E-09 & $1.05 \mathrm{E}-09$ & $8.80 \mathrm{E}-06$ \\
\hline 1.04 & $9.24 \mathrm{E}-06$ & $1.40 \mathrm{E}-05$ & $3.34 \mathrm{E}-07$ & $2.84 \mathrm{E}-08$ & 8.87E-06 & $3.14 \mathrm{E}-09$ & $9.05 \mathrm{E}-10$ & $9.40 \mathrm{E}-06$ \\
\hline 1.02 & 7.06E-06 & $1.70 \mathrm{E}-05$ & $2.75 \mathrm{E}-07$ & $1.53 \mathrm{E}-08$ & $6.77 \mathrm{E}-06$ & $1.14 \mathrm{E}-09$ & $3.81 \mathrm{E}-10$ & 8.80E-06 \\
\hline 1.00 & 7.41E-06 & $2.20 \mathrm{E}-05$ & $2.44 \mathrm{E}-07$ & $1.00 \mathrm{E}-08$ & 7.15E-06 & $9.28 \mathrm{E}-10$ & $2.38 \mathrm{E}-10$ & $1.50 \mathrm{E}-05$ \\
\hline 0.99 & $1.04 \mathrm{E}-05$ & $2.20 \mathrm{E}-05$ & 4.02E-07 & $1.62 \mathrm{E}-08$ & 9.96E-06 & $1.20 \mathrm{E}-09$ & $2.86 \mathrm{E}-10$ & $1.30 \mathrm{E}-05$ \\
\hline 0.96 & $8.68 \mathrm{E}-06$ & $3.00 \mathrm{E}-05$ & $3.47 \mathrm{E}-07$ & $9.06 \mathrm{E}-09$ & $8.32 \mathrm{E}-06$ & $6.86 \mathrm{E}-10$ & $1.90 \mathrm{E}-10$ & $1.30 \mathrm{E}-05$ \\
\hline 0.96 & $9.64 \mathrm{E}-06$ & $2.40 \mathrm{E}-05$ & 4.76E-07 & $1.16 \mathrm{E}-08$ & $9.15 \mathrm{E}-06$ & $1.14 \mathrm{E}-09$ & $2.86 \mathrm{E}-10$ & $1.30 \mathrm{E}-05$ \\
\hline 0.97 & $1.07 \mathrm{E}-05$ & $1.90 \mathrm{E}-05$ & $3.29 \mathrm{E}-07$ & $1.26 \mathrm{E}-08$ & $1.03 \mathrm{E}-05$ & $7.43 \mathrm{E}-10$ & $2.86 \mathrm{E}-10$ & $1.30 \mathrm{E}-05$ \\
\hline 1.06 & $9.12 \mathrm{E}-06$ & $2.10 \mathrm{E}-05$ & 3.02E-07 & $1.16 \mathrm{E}-08$ & $8.81 \mathrm{E}-06$ & $4.76 \mathrm{E}-10$ & $1.90 \mathrm{E}-10$ & $1.40 \mathrm{E}-05$ \\
\hline 1.09 & $1.20 \mathrm{E}-05$ & $2.20 \mathrm{E}-05$ & 4.43E-07 & $1.65 \mathrm{E}-08$ & $1.16 \mathrm{E}-05$ & $1.09 \mathrm{E}-09$ & $2.86 \mathrm{E}-10$ & $1.40 \mathrm{E}-05$ \\
\hline 1.06 & $1.25 \mathrm{E}-05$ & $1.80 \mathrm{E}-05$ & 5.61E-07 & $1.52 \mathrm{E}-08$ & $1.19 \mathrm{E}-05$ & $1.23 \mathrm{E}-09$ & $2.86 \mathrm{E}-10$ & $1.30 \mathrm{E}-05$ \\
\hline 1.06 & $1.10 \mathrm{E}-05$ & $2.40 \mathrm{E}-05$ & 4.05E-07 & $1.53 \mathrm{E}-08$ & $1.06 \mathrm{E}-05$ & $5.86 \mathrm{E}-10$ & 3.33E-10 & $1.20 \mathrm{E}-05$ \\
\hline 1.06 & 8.54E-06 & $2.00 \mathrm{E}-05$ & 2.47E-07 & $1.30 \mathrm{E}-08$ & $8.28 \mathrm{E}-06$ & $6.86 \mathrm{E}-10$ & 3.33E-10 & $1.20 \mathrm{E}-05$ \\
\hline
\end{tabular}




\section{Appendix $\mathrm{H}$ \\ MRM Can L1303 Reduction Calculation using the Fill Pressure and RGA Partial Pressure of Argon}




\begin{tabular}{|c|c|c|c|c|c|c|c|c|c|c|c|c|c|}
\hline \multirow[t]{2}{*}{ Method } & \multicolumn{2}{|c|}{ PAr Pressure } & \multicolumn{6}{|c|}{ Data from executed procedures } & \multicolumn{5}{|c|}{ Partial Pressure calculations } \\
\hline & & & $\begin{array}{l}\text { Manifold } \\
\text { pressure }\end{array}$ & $\begin{array}{l}\text { Man rel } \\
A, B \text { or } C\end{array}$ & $\begin{array}{l}\text { Man rel } \\
A, B \text { or } C\end{array}$ & $\begin{array}{l}\text { Release } \\
\text { pres }\end{array}$ & $\begin{array}{l}\text { Calc } \\
\mathrm{Pr}\end{array}$ & $\mathrm{CO} \mathrm{Pr}$ & $\begin{array}{l}\text { Cal CO } \\
\text { (Ar P) }\end{array}$ & $\begin{array}{l}\text { Cal He } \\
\text { (Ar P) }\end{array}$ & $\begin{array}{c}\text { Calc } \mathrm{CO}_{2} \\
\text { (Ar P) }\end{array}$ & $\begin{array}{l}\mathrm{CO}+\mathrm{He} \\
(\mathrm{ArP})\end{array}$ & $\begin{array}{l}\text { Tmax } \\
\text { (Ar P) }\end{array}$ \\
\hline \multirow[t]{2}{*}{ Date } & $\begin{array}{l}\text { Manifold } \\
\text { / Station }\end{array}$ & & psia & psia & psia & psia & psia & $\mathrm{Pa}$ & $\mathrm{Pa}$ & $\mathrm{Pa}$ & $\mathrm{Pa}$ & psia & $\mathrm{K}$ \\
\hline & & & & & & & & & 20 & 20 & 20 & & \\
\hline $5 / 31 / 2017$ & $A / 3$ & L1303 & 21.17 & & 7.42 & 7.45 & 21.16 & 2896 & 4815 & 2824 & 23 & 1.11 & 1097 \\
\hline $6 / 8 / 2017$ & $A / 3$ & L1303 & 20.01 & & 7.02 & 7.00 & 20.01 & 222 & 305 & 373 & 29 & 0.10 & 841 \\
\hline $6 / 14 / 2017$ & $A / 3$ & L1303 & 19.96 & & 7.03 & 6.92 & 19.98 & 136 & 228 & 324 & 31 & 0.08 & 819 \\
\hline $6 / 21 / 2017$ & $A / 3$ & L1303 & & 6.78 & 2.42 & 2.36 & 20.04 & 156 & 182 & 910 & 25 & 0.16 & 811 \\
\hline $6 / 28 / 2017$ & $A / 3$ & L1303 & & 6.78 & 2.43 & 2.34 & 20.02 & 117 & 396 & 2264 & 44 & 0.39 & 844 \\
\hline $7 / 6 / 2017$ & $A / 3$ & L1303 & & 6.78 & 2.42 & 2.37 & 20.01 & 123 & 224 & 1402 & 31 & 0.24 & 817 \\
\hline $7 / 12 / 2017$ & $A / 3$ & L1303 & & 6.79 & 2.43 & 2.38 & 20.05 & 116 & 116 & 643 & 16 & 0.11 & 797 \\
\hline $7 / 19 / 2017$ & $A / 3$ & L1303 & & 6.76 & 2.42 & 2.34 & 20.01 & 130 & 116 & 664 & 21 & 0.11 & 788 \\
\hline $7 / 25 / 2017$ & $A / 3$ & L1303 & & 6.76 & 2.42 & 2.37 & 20.06 & 107 & 103 & 594 & 8 & 0.10 & 813 \\
\hline $8 / 2 / 2017$ & $A / 3$ & L1303 & & 6.78 & 2.43 & 2.38 & 20.02 & 109 & 159 & 1285 & 19 & 0.21 & 810 \\
\hline $8 / 9 / 2017$ & $A / 3$ & L1303 & & 6.75 & 2.42 & 2.33 & 20.02 & 118 & 161 & 1122 & 22 & 0.19 & 807 \\
\hline $8 / 16 / 2017$ & $A / 3$ & L1303 & & 6.78 & 2.42 & 2.36 & 20.05 & 116 & 156 & 1069 & 17 & 0.18 & 813 \\
\hline $8 / 24 / 2017$ & $A / 3$ & L1303 & & 6.48 & 2.32 & 2.26 & 20.00 & 116 & 159 & 1175 & 16 & 0.19 & 817 \\
\hline $11 / 6 / 2017$ & $A / 3$ & L1303 & & 8.32 & 2.95 & 2.89 & 20.04 & 12952 & 32812 & 12264 & 53 & 6.54 & 1308 \\
\hline $12 / 6 / 2017$ & $A / 3$ & L1303 & & 6.65 & 2.38 & 2.32 & 20.07 & 701 & 1114 & 5279 & 52 & 0.93 & 915 \\
\hline $1 / 10 / 2018$ & $A / 3$ & L1303 & & 6.62 & 2.34 & 2.28 & 20.05 & 347 & 507 & 5986 & 64 & 0.94 & 848 \\
\hline $2 / 7 / 2018$ & $A / 3$ & L1303 & & 6.67 & 2.36 & 2.26 & 20.03 & 281 & 442 & 5189 & 49 & 0.82 & 848 \\
\hline $3 / 5 / 2018$ & $A / 3$ & L1303 & & 6.26 & 2.26 & 2.22 & 20.00 & 124 & 312 & 5591 & 23 & 0.86 & 850 \\
\hline $4 / 4 / 2018$ & $c / 27$ & L1303 & & 6.84 & 2.65 & 2.61 & 20.09 & 63 & 194 & 4730 & 18 & 0.71 & 826 \\
\hline $5 / 2 / 2018$ & $C / 27$ & L1303 & & 5.64 & 2.20 & 2.21 & 20.01 & 102 & 224 & 5569 & 17 & 0.84 & 838 \\
\hline $6 / 6 / 2018$ & $C / 27$ & L1303 & & 5.64 & 2.17 & 2.22 & 20.02 & 42 & 150 & 5753 & 11 & 0.86 & 824 \\
\hline $7 / 11 / 2018$ & $c / 27$ & L1303 & & 5.62 & 2.16 & 2.21 & 20.00 & 67 & 175 & 7165 & 17 & 1.06 & 821 \\
\hline $8 / 7 / 2018$ & $C / 27$ & L1303 & & 5.63 & 2.17 & 2.20 & 20.00 & 91 & 168 & 4396 & 10 & 0.66 & 836 \\
\hline $9 / 6 / 2018$ & $C / 27$ & L1303 & & 5.69 & 2.27 & 2.16 & 20.07 & 76 & 182 & 4736 & 7 & 0.71 & 852 \\
\hline $10 / 4 / 2018$ & $C / 27$ & L1303 & & 5.57 & 2.27 & 2.14 & 19.98 & 103 & 196 & 5275 & 13 & 0.79 & 838 \\
\hline $11 / 12 / 2018$ & $\mathrm{C} / 27$ & L1303 & & 5.79 & 2.32 & 2.21 & 20.06 & 117 & 177 & 6533 & 14 & 0.97 & 828 \\
\hline $12 / 12 / 2018$ & $\mathrm{C} / 27$ & L1303 & & 5.73 & 2.30 & 2.20 & 20.06 & 88 & 200 & 5272 & 8 & 0.79 & 858 \\
\hline $1 / 9 / 2019$ & $C / 27$ & L1303 & & 5.80 & 2.33 & 2.23 & 20.06 & 90 & 217 & 4120 & 11 & 0.63 & 849 \\
\hline
\end{tabular}




\begin{tabular}{|c|c|c|c|c|c|c|c|c|c|c|}
\hline \multicolumn{4}{|c|}{ Release Pressures } & \multicolumn{4}{|c|}{ Can pressure - double release } & \multirow[b]{2}{*}{ Can Temp } & \multirow{3}{*}{$\begin{array}{c}\text { Total } \\
\text { pressure } \\
(\mathrm{CO}+\mathrm{HE} \\
+\mathrm{Ar})\end{array}$} & \multirow{3}{*}{$\begin{array}{c}\mathrm{P}(\text { calc } \\
\text { /sum } \\
\text { gas }\end{array}$} \\
\hline $\begin{array}{l}\text { Manifold } \\
\text { pressure }\end{array}$ & $\begin{array}{c}\text { Man Prel } \\
A, B \text { or } C\end{array}$ & $\begin{array}{l}\text { Man rel } \\
\text { A, B or C }\end{array}$ & $\begin{array}{c}\text { Release } \\
\text { pres }\end{array}$ & Variable $(\mathrm{T} / \mathrm{V})$ & $\begin{array}{c}\text { N (MRM } \\
\text { can) }\end{array}$ & $\mathrm{P}$ (calc) & $\mathrm{P}$ (calc) & & & \\
\hline $\mathrm{Pa}$ & $\mathrm{Pa}$ & $\mathrm{Pa}$ & $\mathrm{Pa}$ & & & $\mathrm{Pa}$ & psia & $\mathrm{K}$ & & \\
\hline 145922 & 0 & 51145 & 51352 & & & & & 558 & 21.17 & \\
\hline 137926 & 0 & 48388 & 48250 & & & & & 563 & 20.11 & \\
\hline 137581 & 0 & 48457 & 47699 & & & & & 561 & 20.06 & \\
\hline 0 & 46734 & 16681 & 16267 & 294329 & $5.28 \mathrm{E}-02$ & 129315 & 18.76 & 559 & 20.20 & 0.93 \\
\hline 0 & 46734 & 16750 & 16129 & 289176 & $5.32 \mathrm{E}-02$ & 127869 & 18.55 & 558 & 20.41 & 0.91 \\
\hline 0 & 46734 & 16681 & 16336 & 294329 & $5.28 \mathrm{E}-02$ & 129315 & 18.76 & 558 & 20.25 & 0.93 \\
\hline 0 & 46803 & 16750 & 16405 & 291002 & $5.31 \mathrm{E}-02$ & 128570 & 18.65 & 558 & 20.16 & 0.93 \\
\hline 0 & 46596 & 16681 & 16129 & 290623 & $5.29 \mathrm{E}-02$ & 127896 & 18.55 & 560 & 20.12 & 0.92 \\
\hline 0 & 46596 & 16681 & 16336 & 290623 & $5.29 \mathrm{E}-02$ & 127896 & 18.55 & 559 & 20.16 & 0.92 \\
\hline 0 & 46734 & 16750 & 16405 & 289176 & $5.32 \mathrm{E}-02$ & 127869 & 18.55 & 559 & 20.23 & 0.92 \\
\hline 0 & 46527 & 16681 & 16060 & 288793 & $5.30 \mathrm{E}-02$ & 127196 & 18.45 & 558 & 20.21 & 0.91 \\
\hline 0 & 46734 & 16681 & 16267 & 294329 & $5.28 \mathrm{E}-02$ & 129315 & 18.76 & 559 & 20.23 & 0.93 \\
\hline 0 & 44666 & 15991 & 15578 & 290497 & $5.07 \mathrm{E}-02$ & 122565 & 17.78 & 559 & 20.19 & 0.88 \\
\hline 0 & 57349 & 20334 & 19920 & 302934 & $6.42 \mathrm{E}-02$ & 161650 & 23.45 & 558 & 26.58 & 0.88 \\
\hline 0 & 45838 & 16405 & 15991 & 290948 & $5.20 \mathrm{E}-02$ & 125905 & 18.27 & 559 & 21.00 & 0.87 \\
\hline 0 & 45631 & 16129 & 15716 & 307060 & $5.08 \mathrm{E}-02$ & 129751 & 18.82 & 562 & 20.99 & 0.90 \\
\hline 0 & 45975 & 16267 & 15578 & 305733 & $5.13 \mathrm{E}-02$ & 130364 & 18.91 & 564 & 20.85 & 0.91 \\
\hline 0 & 43149 & 15578 & 15302 & 280414 & 4.97E-02 & 115792 & 16.80 & 564 & 20.86 & 0.81 \\
\hline 0 & 47147 & 18266 & 17990 & 322703 & $6.07 \mathrm{E}-02$ & 162985 & 23.65 & 563 & 20.80 & 1.14 \\
\hline 0 & 38876 & 15164 & 15233 & 310686 & 5.07E-02 & 130834 & 18.98 & 561 & 20.85 & 0.91 \\
\hline 0 & 38876 & 14958 & 15302 & 335728 & $4.95 \mathrm{E}-02$ & 138246 & 20.06 & 562 & 20.88 & 0.96 \\
\hline 0 & 38738 & 14889 & 15233 & 337808 & $4.93 \mathrm{E}-02$ & 138369 & 20.07 & 561 & 21.06 & 0.95 \\
\hline 0 & 38807 & 14958 & 15164 & 332313 & $4.96 \mathrm{E}-02$ & 136992 & 19.87 & 561 & 20.66 & 0.96 \\
\hline 0 & 39220 & 15647 & 14889 & 275580 & $5.30 \mathrm{E}-02$ & 121511 & 17.63 & 560 & 20.78 & 0.85 \\
\hline 0 & 38393 & 15647 & 14751 & 247677 & $5.38 \mathrm{E}-02$ & 110792 & 16.07 & 554 & 20.77 & 0.77 \\
\hline 0 & 39910 & 15991 & 15233 & 269485 & $5.44 \mathrm{E}-02$ & 121795 & 17.67 & 554 & 21.03 & 0.84 \\
\hline 0 & 39496 & 15854 & 15164 & 267088 & $5.40 \mathrm{E}-02$ & 119812 & 17.38 & 552 & 20.85 & 0.83 \\
\hline 0 & 39979 & 16060 & 15371 & 265986 & 5.47E-02 & 120940 & 17.55 & 552 & 20.69 & 0.85 \\
\hline & & & & 294601 & Avverage & & & & Average & 0.90 \\
\hline
\end{tabular}




\begin{tabular}{|c|c|c|c|c|c|c|c|c|c|c|c|c|c|c|}
\hline \multicolumn{7}{|c|}{ Reduction Calculation } & \multicolumn{8}{|c|}{ RGA Data } \\
\hline $\begin{array}{c}\text { \# mol } \\
\text { gas }\end{array}$ & $\# \mathrm{~mol} \mathrm{CO}$ & $\begin{array}{c}\# \mathrm{~mol} \\
\mathrm{CO} 2\end{array}$ & $\begin{array}{c}\# \text { mol of } \\
\text { "O" }\end{array}$ & & & & & & & & & & & \\
\hline $\mathrm{mol}$ & $\mathrm{mol}$ & $\mathrm{mol}$ & & $\begin{array}{c}\mathrm{PuO} 2 \\
\mathrm{~mol}\end{array}$ & PuOx & $\begin{array}{c}\text { Ratio } \\
\text { of } \\
\text { mol }\end{array}$ & $\begin{array}{l}\text { Total } \\
\text { pressure }\end{array}$ & torr & $\begin{array}{l}\text { Total } \\
\text { press }\end{array}$ & Ch 1 & $\mathrm{Ch} 2$ & Ch 3 & Ch 4 & Ch 12 \\
\hline & & & & & & & $\mathrm{Pa}$ & & AMU/chg & 4 & 28 & 40 & 44 & 14 \\
\hline 1.64E-01 & $5.44 \mathrm{E}-03$ & $2.80 \mathrm{E}-05$ & $5.50 \mathrm{E}-03$ & 4.47 & 1.9975 & & 145519 & $5.22 \mathrm{E}-06$ & $8.70 \mathrm{E}-06$ & $1.01 \mathrm{E}-07$ & $1.73 \mathrm{E}-07$ & $4.95 \mathrm{E}-06$ & $8.43 \mathrm{E}-10$ & 6.67E-10 \\
\hline $1.57 \mathrm{E}-01$ & $3.45 \mathrm{E}-04$ & $3.26 \mathrm{E}-05$ & $4.11 \mathrm{E}-04$ & 4.47 & 1.9974 & & 138564 & $6.20 \mathrm{E}-06$ & $8.50 \mathrm{E}-06$ & $1.67 \mathrm{E}-08$ & $1.37 \mathrm{E}-08$ & $6.17 \mathrm{E}-06$ & $1.29 \mathrm{E}-09$ & $2.86 \mathrm{E}-10$ \\
\hline $1.56 \mathrm{E}-01$ & $2.58 \mathrm{E}-04$ & $3.51 \mathrm{E}-05$ & $3.28 \mathrm{E}-04$ & 4.47 & 1.9972 & & 138441 & 5.97E-06 & $1.00 \mathrm{E}-05$ & $1.40 \mathrm{E}-08$ & $9.85 \mathrm{E}-09$ & $5.94 \mathrm{E}-06$ & 1.33E-09 & $2.86 \mathrm{E}-10$ \\
\hline $5.67 \mathrm{E}-02$ & $7.42 \mathrm{E}-05$ & $1.03 \mathrm{E}-05$ & $9.49 \mathrm{E}-05$ & 4.47 & 1.9972 & 0.93 & 138974 & $8.63 \mathrm{E}-06$ & $1.00 \mathrm{E}-05$ & $5.64 \mathrm{E}-08$ & $1.13 \mathrm{E}-08$ & $8.56 \mathrm{E}-06$ & 1.57E-09 & $1.90 \mathrm{E}-10$ \\
\hline $5.74 \mathrm{E}-02$ & 1.62E-04 & $1.82 \mathrm{E}-05$ & $1.98 \mathrm{E}-04$ & 4.47 & 1.9971 & 0.93 & 140562 & $3.31 \mathrm{E}-06$ & $1.10 \mathrm{E}-05$ & $5.33 \mathrm{E}-08$ & 9.33E-09 & $3.25 \mathrm{E}-06$ & 1.05E-09 & $2.86 \mathrm{E}-10$ \\
\hline $5.70 \mathrm{E}-02$ & $9.16 \mathrm{E}-05$ & 1.27E-05 & 1.17E-04 & 4.47 & 1.9970 & 0.93 & 139514 & $5.55 \mathrm{E}-06$ & $1.00 \mathrm{E}-05$ & 5.57E-08 & 8.91E-09 & $5.48 \mathrm{E}-06$ & $1.24 \mathrm{E}-09$ & $2.38 \mathrm{E}-10$ \\
\hline $5.66 \mathrm{E}-02$ & 4.73E-05 & $6.52 \mathrm{E}-06$ & $6.03 \mathrm{E}-05$ & 4.47 & 1.9970 & 0.94 & 138632 & $9.94 \mathrm{E}-06$ & $9.90 \mathrm{E}-06$ & $4.60 \mathrm{E}-08$ & $8.28 \mathrm{E}-09$ & $9.88 \mathrm{E}-06$ & $1.14 \mathrm{E}-09$ & $2.38 \mathrm{E}-10$ \\
\hline $5.66 \mathrm{E}-02$ & 4.72E-05 & $8.61 \mathrm{E}-06$ & $6.44 \mathrm{E}-05$ & 4.47 & 1.9970 & 0.94 & 138658 & $1.13 \mathrm{E}-05$ & $1.00 \mathrm{E}-05$ & $5.40 \mathrm{E}-08$ & $9.40 \mathrm{E}-09$ & $1.12 \mathrm{E}-05$ & 1.71E-09 & $1.43 \mathrm{E}-10$ \\
\hline $5.66 \mathrm{E}-02$ & 4.19E-05 & $3.10 \mathrm{E}-06$ & $4.81 \mathrm{E}-05$ & 4.47 & 1.9969 & 0.94 & 138562 & $1.04 \mathrm{E}-05$ & $1.00 \mathrm{E}-05$ & 4.47E-08 & 7.71E-09 & $1.04 \mathrm{E}-05$ & $5.71 \mathrm{E}-10$ & $2.38 \mathrm{E}-10$ \\
\hline 5.69E-02 & $6.50 \mathrm{E}-05$ & $7.95 \mathrm{E}-06$ & 8.09E-05 & 4.47 & 1.9969 & 0.94 & 139321 & $6.82 \mathrm{E}-06$ & $9.90 \mathrm{E}-06$ & $6.28 \mathrm{E}-08$ & $7.78 \mathrm{E}-09$ & $6.75 \mathrm{E}-06$ & $9.52 \mathrm{E}-10$ & $9.52 \mathrm{E}-11$ \\
\hline $5.68 \mathrm{E}-02$ & $6.56 \mathrm{E}-05$ & $8.93 \mathrm{E}-06$ & $8.35 \mathrm{E}-05$ & 4.47 & 1.9969 & 0.93 & 139162 & $7.27 \mathrm{E}-06$ & $9.80 \mathrm{E}-06$ & $5.86 \mathrm{E}-08$ & $8.39 \mathrm{E}-09$ & $7.20 \mathrm{E}-06$ & $1.14 \mathrm{E}-09$ & $1.43 \mathrm{E}-10$ \\
\hline $5.68 \mathrm{E}-02$ & $6.38 \mathrm{E}-05$ & $7.05 \mathrm{E}-06$ & $7.79 \mathrm{E}-05$ & 4.46 & 1.9968 & 0.93 & 139099 & $7.45 \mathrm{E}-06$ & $1.00 \mathrm{E}-05$ & $5.71 \mathrm{E}-08$ & 8.36E-09 & 7.39E-06 & $9.24 \mathrm{E}-10$ & $1.43 \mathrm{E}-10$ \\
\hline $5.68 \mathrm{E}-02$ & $6.49 \mathrm{E}-05$ & $6.46 \mathrm{E}-06$ & $7.79 \mathrm{E}-05$ & 4.46 & 1.9968 & 0.89 & 139207 & $8.12 \mathrm{E}-06$ & $1.10 \mathrm{E}-05$ & $6.86 \mathrm{E}-08$ & $9.28 \mathrm{E}-09$ & $8.05 E-06$ & $9.24 \mathrm{E}-10$ & $1.90 \mathrm{E}-10$ \\
\hline 7.47E-02 & $1.34 \mathrm{E}-02$ & $2.16 \mathrm{E}-05$ & $1.34 \mathrm{E}-02$ & 4.45 & 1.9908 & 0.86 & 182986 & $7.85 \mathrm{E}-06$ & $1.50 \mathrm{E}-05$ & $5.26 \mathrm{E}-07$ & $1.41 \mathrm{E}-06$ & $5.92 \mathrm{E}-06$ & $2.27 \mathrm{E}-09$ & $4.48 \mathrm{E}-09$ \\
\hline 5.89E-02 & $4.55 \mathrm{E}-04$ & $2.14 \mathrm{E}-05$ & $4.98 \mathrm{E}-04$ & 4.45 & 1.9906 & 0.88 & 144303 & $8.55 \mathrm{E}-06$ & $1.30 \mathrm{E}-05$ & $3.12 \mathrm{E}-07$ & $6.58 \mathrm{E}-08$ & 8.17E-06 & 3.09E-09 & 1.00E-09 \\
\hline $5.90 \mathrm{E}-02$ & $2.07 \mathrm{E}-04$ & $2.62 \mathrm{E}-05$ & $2.59 \mathrm{E}-04$ & 4.45 & 1.9905 & 0.86 & 144414 & $9.31 \mathrm{E}-06$ & $1.30 \mathrm{E}-05$ & $3.85 \mathrm{E}-07$ & $3.26 \mathrm{E}-08$ & $8.89 \mathrm{E}-06$ & 4.13E-09 & $1.05 \mathrm{E}-09$ \\
\hline $5.86 \mathrm{E}-02$ & $1.81 \mathrm{E}-04$ & $2.00 \mathrm{E}-05$ & $2.21 \mathrm{E}-04$ & 4.45 & 1.9904 & 0.88 & 143537 & $9.24 \mathrm{E}-06$ & 1.40E-05 & $3.34 \mathrm{E}-07$ & $2.84 \mathrm{E}-08$ & 8.87E-06 & $3.14 \mathrm{E}-09$ & $9.05 \mathrm{E}-10$ \\
\hline $5.87 \mathrm{E}-02$ & 1.27E-04 & $9.49 \mathrm{E}-06$ & 1.46E-04 & 4.45 & 1.9903 & 0.85 & 143783 & $7.06 \mathrm{E}-06$ & $1.70 \mathrm{E}-05$ & $2.75 \mathrm{E}-07$ & $1.53 \mathrm{E}-08$ & $6.77 \mathrm{E}-06$ & $1.14 \mathrm{E}-09$ & $3.81 \mathrm{E}-10$ \\
\hline $5.83 \mathrm{E}-02$ & $7.92 \mathrm{E}-05$ & $7.34 \mathrm{E}-06$ & $9.39 \mathrm{E}-05$ & 4.45 & 1.9903 & 1.04 & 142799 & $7.41 \mathrm{E}-06$ & $2.20 \mathrm{E}-05$ & $2.44 \mathrm{E}-07$ & $1.00 \mathrm{E}-08$ & $7.15 \mathrm{E}-06$ & $9.28 \mathrm{E}-10$ & $2.38 \mathrm{E}-10$ \\
\hline 5.87E-02 & $9.15 \mathrm{E}-05$ & $6.78 \mathrm{E}-06$ & $1.05 \mathrm{E}-04$ & 4.45 & 1.9902 & 0.86 & 143667 & $1.04 \mathrm{E}-05$ & $2.20 \mathrm{E}-05$ & $4.02 \mathrm{E}-07$ & $1.62 \mathrm{E}-08$ & 9.96E-06 & $1.20 \mathrm{E}-09$ & $2.86 \mathrm{E}-10$ \\
\hline $5.87 \mathrm{E}-02$ & $6.13 \mathrm{E}-05$ & 4.64E-06 & $7.06 \mathrm{E}-05$ & 4.45 & 1.9902 & 0.84 & 143771 & $8.68 \mathrm{E}-06$ & $3.00 \mathrm{E}-05$ & 3.47E-07 & $9.06 \mathrm{E}-09$ & $8.32 \mathrm{E}-06$ & $6.86 \mathrm{E}-10$ & $1.90 \mathrm{E}-10$ \\
\hline $5.93 \mathrm{E}-02$ & $7.15 \mathrm{E}-05$ & $7.03 E-06$ & $8.55 \mathrm{E}-05$ & 4.45 & 1.9901 & 0.83 & 145214 & $9.64 \mathrm{E}-06$ & $2.40 \mathrm{E}-05$ & $4.76 \mathrm{E}-07$ & $1.16 \mathrm{E}-08$ & $9.15 \mathrm{E}-06$ & $1.14 \mathrm{E}-09$ & $2.86 \mathrm{E}-10$ \\
\hline $5.82 \mathrm{E}-02$ & $6.86 \mathrm{E}-05$ & $4.05 \mathrm{E}-06$ & 7.67E-05 & 4.45 & 1.9901 & 0.85 & 142431 & $1.07 \mathrm{E}-05$ & $1.90 \mathrm{E}-05$ & $3.29 \mathrm{E}-07$ & $1.26 \mathrm{E}-08$ & $1.03 \mathrm{E}-05$ & $7.43 \mathrm{E}-10$ & $2.86 \mathrm{E}-10$ \\
\hline $5.83 \mathrm{E}-02$ & $7.43 \mathrm{E}-05$ & $3.05 \mathrm{E}-06$ & $8.04 \mathrm{E}-05$ & 4.45 & 1.9901 & 0.91 & 142783 & $9.12 \mathrm{E}-06$ & $2.10 \mathrm{E}-05$ & $3.02 \mathrm{E}-07$ & $1.16 \mathrm{E}-08$ & $8.81 \mathrm{E}-06$ & $4.76 \mathrm{E}-10$ & $1.90 \mathrm{E}-10$ \\
\hline $5.85 \mathrm{E}-02$ & $8.01 \mathrm{E}-05$ & $5.32 \mathrm{E}-06$ & $9.08 \mathrm{E}-05$ & 4.45 & 1.9900 & 0.92 & 143341 & $1.20 \mathrm{E}-05$ & $2.20 \mathrm{E}-05$ & $4.43 \mathrm{E}-07$ & $1.65 \mathrm{E}-08$ & $1.16 \mathrm{E}-05$ & 1.09E-09 & $2.86 \mathrm{E}-10$ \\
\hline $5.90 \mathrm{E}-02$ & $7.24 \mathrm{E}-05$ & $5.84 \mathrm{E}-06$ & $8.40 \mathrm{E}-05$ & 4.45 & 1.9900 & 0.92 & 144582 & $1.25 \mathrm{E}-05$ & $1.80 \mathrm{E}-05$ & 5.61E-07 & $1.52 \mathrm{E}-08$ & 1.19E-05 & $1.23 \mathrm{E}-09$ & $2.86 \mathrm{E}-10$ \\
\hline $5.85 \mathrm{E}-02$ & $8.15 \mathrm{E}-05$ & $3.11 \mathrm{E}-06$ & $8.78 \mathrm{E}-05$ & 4.45 & 1.9899 & 0.92 & 143336 & $1.10 \mathrm{E}-05$ & $2.40 \mathrm{E}-05$ & $4.05 \mathrm{E}-07$ & $1.53 \mathrm{E}-08$ & $1.06 \mathrm{E}-05$ & $5.86 \mathrm{E}-10$ & $3.33 \mathrm{E}-10$ \\
\hline $5.81 \mathrm{E}-02$ & $8.86 \mathrm{E}-05$ & 4.67E-06 & $9.79 \mathrm{E}-05$ & 4.45 & 1.9899 & 0.94 & 142205 & $8.54 \mathrm{E}-06$ & $2.00 \mathrm{E}-05$ & $2.47 \mathrm{E}-07$ & $1.30 \mathrm{E}-08$ & $8.28 \mathrm{E}-06$ & $6.86 \mathrm{E}-10$ & $3.33 \mathrm{E}-10$ \\
\hline
\end{tabular}

